O EMPREGO DOS ESQUEMAS FATORIAIS FRACIONADOS

E DO CONFUNDIMENTO NA EXPERIMENTAÇ̃O

\title{
GILSON MEDEIROS E SILVA
}

(Bacharel em Estatistica).

ORIENTADOR: PROF, DR, ROBERTO SIMIONATO MORAES

Dissertação apresentada à Escola Superior de Agricultura "Luiz de Queiroz", da universidade de são Paulo, pará obtenção do título de Mestre em Agronomia - Area de Concentração: Estatistica e Expe rimentação Agronômica.

PIRACICABA

ESTADO DE SÃO PAULO - BRASIL

FEVERE IRO - 1987. 
.ii.

A minha esposa, Angela

Aos meus filhos, Giselle, Jean e Nataly DEDICO 


\section{AGRADECIMENTOS}

- Aos meus pais, pela confiança e estímulo.

- Ao Professor Doutor Roberto Simionato Moraes, pela orienta ção e sugestão, como também pela amizade.

- Ao colega Henri Stuker, pela elaboração do programa.

- Aos professores do Departamento de Matemática e Estatistica da ESALQ, pelos ensinamentos.

- Ao Professor Doutor Humberto de Campos, pelo incentivo e consideração.

- Ao Professor José Amarino Maciel de Brito pelo Summary.

- Aos colegas do Curso de Estatística e Experimentação Agronômica, pela amizade.

- A todos os funcionärios do Departamento de Matemática e Es tatistica da ESALQ, pelos favores prestados.

- A todos aqueles que contribuiram para a realização deste traba1ho.

- Ao desenhista Antonio F1avio Filho, pelos gräficos.

- A Rosa Maria Alves, pelos favores prestados. 
. iv.

\section{INDICE}

pägina

RE SUMO

SUMMARY

1. INTRODUÇÃO

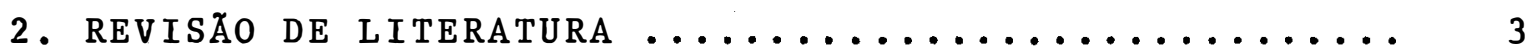

3. METODO E MATERIAL ......................... 20

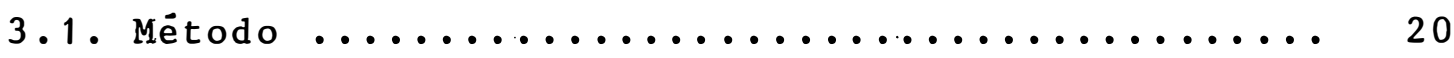

3.1.1. Experimentos Fatoriais ............. 20

3.1.1.1. Experimentos Fatoriais da Sërie

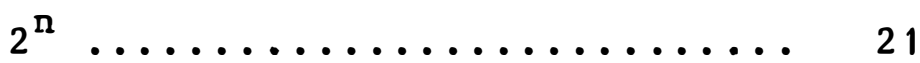

3.1.1.1.1. Modêlo Matemätico ......... 21

3.1.1.1.2. Forma Gera1 de Apresentar os fatoriais $2^{n} \ldots \ldots \ldots \ldots$

3.1.1.1.3. A Anālise de Variância para os Fatoriais da Sërie $2^{\mathrm{n}} \ldots \ldots .28$

3.1.1.2. Experimentos Fatoriais da Série

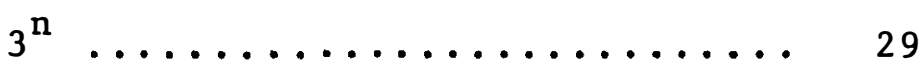

3.1.1.2.1. Modêlo Matemätico ......... 29

3.1.1.2.2. A Forma Geral de Apresentar os Fatoriais $3^{n} \ldots \ldots \ldots$ 
pägina

3.1.1.2.3. Anāilse de Variāncia para o Fatoria1 da Série $3^{n} \ldots \ldots$

3.1.2. Confundimento nos Experimentos Fato-

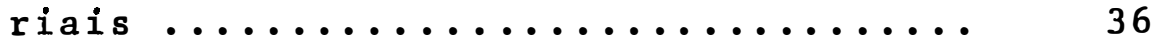

3.1.2.1. Confundimento na Série $2^{n} \ldots 37$

3.1.2.2. Confundimento na Sërie $3^{n} \ldots 50$

3.1.3. Experimentos Fatoriais com uma ünica

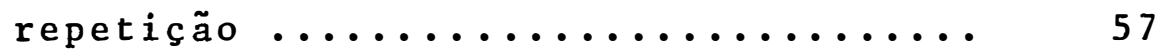

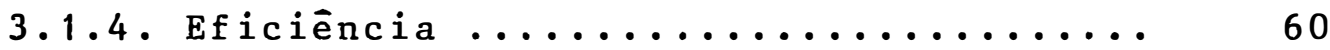

3.1.4.1. No Caso de uma Ûnica Repeti-

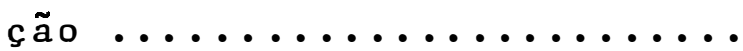

60

3.1.4.2. No Caso de mais de uma Repeti ção $\ldots \ldots \ldots \ldots \ldots \ldots \ldots \ldots \ldots \ldots \ldots$

3.1:5. Fatoria 1 Fracionado ............... 62

3.1.5.1. Fatoria 1 Fracionado no sistema $2^{n} \ldots \ldots \ldots \ldots \ldots$

3.1.5.1.1. Descrição do Bloco Principa1, fração $2^{n-k}$ e Conjunto

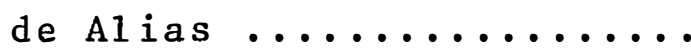

3.1.5.1.2. Fatoriais Fracionados da Sé rie $2^{\mathrm{n}}$ em Blocos Ao Acaso..

3.1.5.2. Fatoria1 Fracionado no Sistema $3^{n} \ldots \ldots \ldots \ldots \ldots$

3.1.5.2.1. Fatoriais Fracionados da Sé rie $3^{\text {n }}$ em Blocos ao Acaso.. 
3.1.5.2.2. Observações .......... 108

3.1.6. Método Prätico de Yates ........... 111

3.2. Material ........................... 113

4. RESULTADOS E DISCUSA $\ldots \ldots \ldots \ldots \ldots \ldots \ldots \ldots \ldots \ldots$

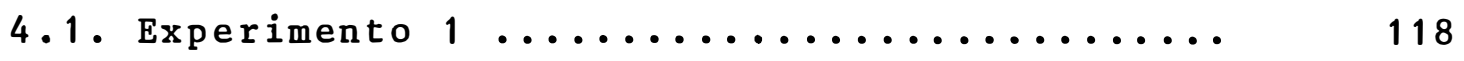

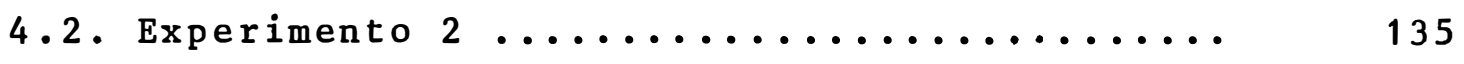

4.3 . Experimento $3 \ldots \ldots \ldots \ldots \ldots \ldots \ldots \ldots \ldots \ldots \ldots$

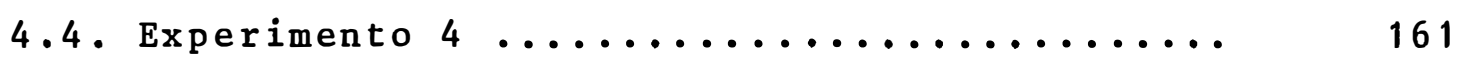

5. concLusons .............................. 176

6. BIBLIOGRAFIA ......................... 178

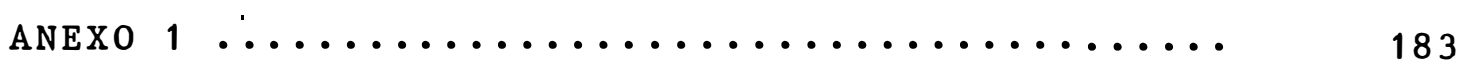

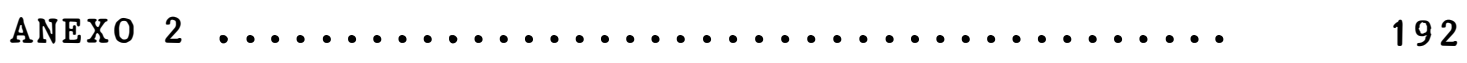




\title{
O EMPREGO DOS ESQUEMAS FATORIAIS FRACIONADOS
}

E DO CONFUNDIMENTO NA EXPERIMENTAÇÃO

\author{
Autor: GILSON MEDEIROS E SILVA \\ Orientador: Prof. Dr. Roberto Simionato Moraes
}

\section{RESUMO}

o objetivo principal deste trabalho é estudar a utilização dos esquemas fatoriais completo com e sem confundimento e do fatorial fracionado com e sem confundimento para as sèries $2^{\mathrm{n}}$ e $3^{\mathrm{n}}$.

Com este intuito apresentamos o desenvolvimen to teórico desses esquemas, empregando a notação mais tradicional como também a geometria finita.

os dados utilizados foram gerados por simulaçao através do modelo matemático inteiramente ao acaso do fatorial $2^{6}$, onde os efeitos principais e as interaçóes foram fixados. Os erros foram tirados de uma tabela com distribuição normal $\operatorname{com} \mu=0$ e $\sigma^{2}=1$. Variamos o desvio padrão dos erros normais e assim estruturamos 4 experimentos. 
Montamos para cada experimento, quadros de anālise de variância sem confundimento, com uma e duas interações confundidas quando o fatorial é completo, e para $1 / 2$ da repetição foi montado quadros sem confundimento e com uma interaça confundida. Também foram calculadas as eficiências.

Com base nestes resultados mostramos as melho res interações para ser confundidas como também as maiores eficiências, e vemos que o fatorial fracionado é mais eficiente do que o fatorial completo.

Também observamos que a eficiència nos casos estudados não mudam de um experimento para outro mesmo que a variância dos erros aumente. 
THE USE OF FRACTIONAL FACTORIAL DESIGNS

AND CONFOUNDING IN EXPERIMENTATION

Author: GILSON MEDEIROS E SILVA

Adviser: Prof. Dr. Roberto Simionato Moraes

\section{SUMMARY}

The main objective of this work is to study the utilization of the complete factorial scheme with and without confounding and of the fractional factorial scheme with and without confounding for the series $2^{n}$ and $3^{n}$.

With this purpose, we present the theoretical development of these schemes, employing the most traditional notation as we 11 as the finite geometry.

The employed data were generated from simulation according to the unique mathematics model at random of the $2^{6}$ factorial, where the main effects and the interations were fixed. The errors had been taken from normal distribution table with $\mu=0$ and $\sigma^{2}=1$. We vary the standard model deflaction of the common errors and then we organize the experiments. 
We built for each experiment tables of variance analyses without confounding, with one and two confused interations when the factorial scheme is complete, and for a half of the repetition was built rables without confounding and with one confused interation. It was also calculated the efficiency.

Based on these results we showed the best interations to be confused as well as the best efficiencies, and then we noticed that the fractional factorial scheme is more efficient than the complete factorial scheme.

We also noticed that, the efficiency, in the studied cases, didn't change from one experiment to the other, even if the errors variance raise. 


\section{INTRODUCÄO}

Em experimentação agrícola, quando värios fatores diferentes são objetos de estudo, um esquema experimen tal altamente eficiente para este estudo è o fatorial comple to, pois além de examinar cada fator, ele estuda o relaciona mento entre os efeitos dos värios fatores.

Mas uma da desvantagem destes esquemas é que o nümero de combinações crescem rapidamente na medida que o número de fatores aumenta, e com isto traz uma série : de problemas para o pesquisador, dentre eles a perda de eficiên cia, pois a medida que aumenta o tamanho do bloco, aumenta a heterogeneidade dentro dele. Também deve ser levado em conta o custo da experimentaça e a viabilidade de se instalar tais experimentos, pois as vezes somente uma repeticão de um experimento pode tornar este ensaio praticamente impossivel de ser realizado. 
Em vista destes problemas busca-se alternativas viáveis para contorna-los. Quando o erro experimental é grande, ou quando se espera um efeito considerável das interações de ordem elevada, não existe nenhuma alternativa. Mas, frequentemente, o erro experimental e as interações de ordem elevada são de mesma grandeza. Em tais casos podemos ter as seguintes alternativas possiveis: primeira, usar o esquema de confundir interações de ordem elevada, que consiste em di vidir cada repetição em värios blocos, perdendo toda informa ção sobre as interações confundidas; segunda, usar o esquema de fatorial fracionado, que consiste em considerar certas in terações como insignificantes (entendendo-se por interações insignificantes aquelas de mesma ordem de grandeza dos efeitos atribuidos ao residuo, e portanto não necessariamente nu 1as), e com isto somente uma parte das combinações é utiliza da no experimento.

Com o exposto acima, este trabalho tem como objetivo fazer algumas comparações entre o fatorial completo, o fatorial confundido e o fatorial fracionado, pois estes delineamentos são de grande interesse prätico na experimentação agricola, e com isto tentar estabelecer qual será o mais indicado. 


\section{REVISÃO DE LITERATURA}

Como a literatura sobre ensaios fatoriais, con fundimento e fatorial fracionado é extensa, nós procuramos selecionar as publicações que mais se aproximam da abordagem dada ao traba1ho.

\section{Assim YATES (1937), apresenta os princípios} bäsicos do delineamento fatorial, tomando como. exemplo o fatorial $2^{3}$.

Sobre o confundimento, ele apresenta o confun dimento total e parcial do fatorial $2^{3}$ em 2 blocos de 4 parcelas cada, e a partir desse fatorial, ele generaliza para a sèrie $2^{\text {n }}$.

Apresenta um exemplo de confundimento do fato rial $2^{5}$, com uma ünica repetição, mostrando como se acha a estimativa do erro por intermédio das interações de ordem e- 
levada. Neste exemplo, ele enfoca o método prático para ca 1 cular os efeitos principais e as interações, como também o ganho em precisão devido ao confundimento.

Tambèm mostra o confundimento do fatorial $2^{3}$ em 2 quadrados latinos $4 \times 4$, e o confundimento dos fatoriais $2^{5}$ e $2^{6}$ em quadrados 1 atinos $8 \times 8$.

Para os fatoriais da série $3^{n}$, apresenta as modificações que são necessārias na anālise estatistica quan do não existe confundimento, através dos fatoriais $3^{2}$ e $3^{3}$. Para esta série, apresenta o confundimento dos seguintes fatoriais: $3^{2}$ em blocos de 3 parcelas, $3^{3}$ em blocos de 9 parce las dando os 4 grupos de confundimento $w, x, y$ e $z$, e $3^{4}$ em blocos de 9 parcelas. A partir desses exemplos faz a extensão em blocos de $3^{n-1}$ ou $3^{n-2}$ parcelas.

Apresenta um método sistemático e um método alternativo para o delineamento $3^{3}$ com uma única repetição.

Para as séries mistas, apresenta exemplos das séries $3 \times 2^{n}$ e $3^{n} \times 2$.

Finalmente, expõe o método de confundimento de fatoriais com 4 e 8 níveis combase na série $2^{n}$.

BOSE e KISHEN (1940), estudam o problema do confundimento em experimentos fatoriais simétricos $\mathrm{s}^{\mathrm{m}}$, onde

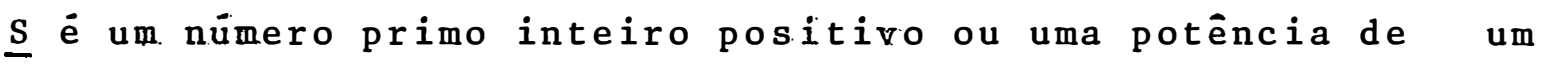


primo, e $\underline{m}$ qualquer número inteiro positivo.

Enunciam o principio da interação generalizada no arranjo fatorial $\mathrm{s}^{\mathrm{m}}$, e usam este importante principio na demonstração de um método geral para formar arranjos confundidos. Com esses arranjos é possivel dividir os $s^{m}-1$ graus de liberdades, pertencentes aos efeitos principais e interações em $\left(s^{m}-1\right) /(s-1)$ conjuntos de $(s-1)$ graus de liberdades.

Mostram que essa divisão é executada identif cando as $\mathrm{s}^{\mathrm{m}}$ combinaçóes de tratamentos com os pontos da Geometria Euclidiana Finita $E G(m, p)$.

FISHER (1942), apresenta o desenvolvimento da relação entre os sistemas. de confundimentos com grupos Abe lianos, para provar uma proposição geral, ligando o tamanho minimo do bloco requerido com o nümero de fatores envolvidos, e fornece um catálogo de sistemas de confundimento até 15 fa tores. 0 desenvolvimento é sobre as séries $2^{n}$ e $p^{n}$, onde $p$ è um nümero primo.

KISHEN (1942), apresenta um método geral de expressar qualquer grau de liberdade para efeitos principais e interações do fatorial $\mathrm{S}^{\mathrm{m}}$ dado por BOSE e KISHEN (1940). Para este estudo, o autor obtém as expressões das componentes para as interações de ordem mais elevadas no caso dos arranjos $3^{4}$ e $4^{3}$. 
FINNEY (1945), introduz. os principios sobre os experimentos fatoriais fracionados, usando a teoria de grupos Abelianos, para os fatoriais das sēries $2^{n}$ e $3^{n}$, onde $\underline{n}$ è um inteiro positivo. Introduz a noção da relação de identidade, mostrando que, se na obtenção de determinada interação todos.os elementos são tomados com o mesmo sinal, ela è igualada à identidade. Fala tambēm em alias e exemplifica através dos esquemas $2^{n-k}$, onde $\underline{n}$ é o nümero de fatores e k è o nümero de geradores de blocos, tomando $n=4,8$ e $k=2,4$ respectivamente. Introduz o conceito de ortogonalidade de dois elementos num mesmo grupo, ou em grupos distintos. So bre os fatoriais da série $3^{n}$, ele apresenta uma discussão anäloga aquela feita para os fatoriais da série $2^{n}$.

FINNEY (1945), apresenta as regras bảsicas pạ ra confundimento, como tambēm o procedimento da formação dos blocos quando uma, duas, etc., interações são confundidas nos fatoriais da série $2^{n}$. Conceitua contraste de definição como sendo as interações confundidas. Tambēm ele discute as melhores frações dos esquemas fatoriais $2^{6}, 2^{7}$ e $2^{8}$, dando os grupos de aliases e suas respectivas anälises de variāncia com seus graus de 1 iberdades.

FISHER (1945), faz uma extensão para os siste mas de confundimento dos fatoriais da série $2^{n}$, desenvolvido no trabalho anterior (1942) para os casos: (i) quando os fatores tem como niveis qualquer número primo, e (ii) para o 
caso em que o nümero de niveis é qualquer potência de um pri mo.

No primeiro caso os fatores podem ser escolhi dos para corresponder com subgrupos de ordem p de um grupo Abelianode ordem $\mathrm{p}^{\mathrm{n}}$, igual ao número de parcelas em icada b1oco. No segundo, cada fator corresponde com uma combinação de s valores, não todos zero, cada um tomando $\mathrm{p}^{\mathrm{r}}$ valores do campo, e sendo este o número de niveis para cada fator.

PLACKETT e BURMAN (1946), apresentam um exemplo de delineamento avancado, mais especificamente para pesquisa fisica e industrial, que são de interesse para o fatorial fracionado, fazendo uma discussão teörica sobre os requisitos para delineamentos ótimos, e os métodos de solução, empregando corpo de Galois.

KEMPTHORNE (1947), apresenta um método geral para os esquemas fatoriais do tipo $\mathrm{p}^{\mathrm{n}}$ a serem examinados, em relação ao confundimento e fatorial fracionados. o método é descrito a partir das regras para os fatoriais da sērie $2^{\text {n }}$ e $3^{\mathrm{n}}$, e corresponde aquelas dadas por FISHER (1942). A presen te abordagem mostra o confundimento e fatorial fracionado co mo aspectos diferentes do mesmo processo.

Sobre confundimento, ele faz uma abordagem somente sobre os fatoriais da série $3^{n}$, dando os seguintes exemplos: 

a. $3^{3}$ em blocos de 9 e 3 tratamentos
b. $3^{4}$ em blocos de 9 tratamentos
c. $3^{5}$ em blocos de 9 tratamentos
d. $3^{6}$ em blocos de 27 tratamentos

Sobre fatorial fracionado, ele faz uma aborda gem extensa sobre a série $2^{\text {n }}$ inteiramente ao acaso, dando as relações de identidade nos seguintes exemplos:
a. $1 / 2$ da repetição do fatorial $2^{n}$, para $n=3,4,5$
b. $1 / 4$ da repetição. do fatorial $2^{n}$, para $n=4,5,6,7,8$
c. $1 / 8$ da repetição do fatorial $2^{n}$, para $\geqq 10$
d. $1 / 16$ da repetição do fatorial $2^{n}$, para $n \geqq 12$

KEMPTHORNE (1947), apresenta os resultados da anālise de uma série de experimento, onde há existència de interações entre tratamentos e blocos, pois está implicitoem todos delineamentos modernos que envolvem confundimento e/ou fatorial fracionado que tais interaçoes são insignificantes, comparado com os efeitos e interações que os experimentos são planejados para estimar.

FINNEY (1947), descreve um método sistemätico de confundimento do fatorial da série $2^{n}$, escolhe uma das interações a serem confundidas comblocos e, pela combinação das letras desta interação, determina os tratamentos do bloco contendo a testemunha (nivel inferior de todos os fatores), denominado de "bloco principal", e constroi através deste os demais blocos. Por extensão deste processo apresen 
ta o confundimento da sërie $3^{n}$.

BROWNLEE, KELLY e LORRAINE (1948), apresentam uma descrição das soluções de valor prático no caso dos expe

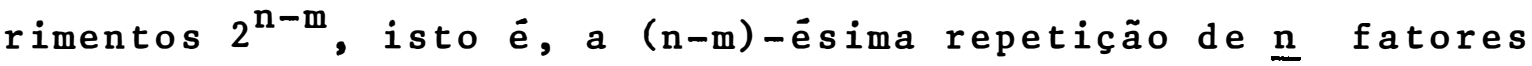
todos em dois niveis, envolvendo o uso de $2^{\mathrm{m}}$ parcelas. Tais arranjos confundido e confundimento duplo serão dados como foram construidos.

Duas soluções üteis são tambēm incluidas onde um e dois fatores respectivamente estão em 4 niveis, estas sendo derivada das soluções com todos fatores em 2 niveis.

Os autores fazem uma discussão sobre os delineamentos baseados em $16,32,64$ e com mais de 64 parcelas.

RAO (1948), apresenta a teoria geral de delineamentos em fatorial fracionado usando as propriedades de formas de potência d. Alguns princípios básicos desta teoria foi dado por KEMPTHORNE (1947) e KISHEN (1947).

DAVIES e HAY (1950), apresentam um método sim ples de construir delineamentos em fatorial fracionados da série $2^{n}$ e discutem o seu uso na pesquisa industrial.

BANERJEE (1950), discute $3 / 4$ de uma repetição do fatorial da série $2^{n}$, mostrando que existe viabilidade pa ra esta fração. Toma como exemplos $3 / 4$ do fatorial $2^{5}$ inte ramente ao acaso e $3 / 4$ do fatorial $2^{6}$ em 6 blocos, dando os 
respectivos quadros da anảlise de variância.

CHINLOY, INNES e FINNEY (1952), apresentam um exemplo numérico $\operatorname{com} 1 / 3$ da repetição do fatorial $3^{5}$ sobre cana-de-açúcar, dando o plano do experimento, a estrutura da anālise de variância, o cálculo das somas de quadrados através de dois métodos sistemático e a interpretação da anảlise de variância.

KEMPTHORNE e TISCHER (1953), apresentam um es tudo em que é feita uma abordagem da determinaçãodos vários aspectos da desidratação do milho verde. Neste trabalho os autores utilizam os princípios dos fatoriaisfracionados da série $2^{\mathrm{n}}$. Para este trabalho foi considerado $1 / 4$ da repetição do fatorial $2^{11}$ em parcelas sub-sub-divididas.

COCHRAN e COX (1957), apresentam as vantagens e desvantagens da utilização dos experimentos fatoriais. Apresentam o método para calcular os efeitos principais e as interações, tomando como base os fatoriais $2^{2}$ e $2^{3}$, e assim generalizam para a série $2^{n}$. Apresentam o método prático de YATES para calcular o efeito total.

Sobre os fatoriais da série $3^{n}$, apresentam um exemplo numérico para o fatorial $3^{2}$ e generalizam o método de anālise para a sèrie.

Os autores apresentam o confundimento total e parcial do fatorial $2^{3}$ em blocos com 4 tratamentos. 
Com relação aos fatoriais da série $3^{n}$, eles apresentam o fatorial $3^{2}$ em blocos com 3 tratamentos, confun dindo 2 graus de 1 iberdade correspondentes a interação $A B$, e o fatorial $3^{3}$ em blocos com 9 tratamentos, confundinco 2 graus de 1 iberdade correspondentes a interação $A B C$.

Para os fatoriais com séries mistas, eles apresentam um exemplo numérico do fatorial $3^{2}$ x 2 em blocos com 6 tratamentos, confundindo parcialmente $A B$ e ABC.

Tambèm eles enfocam o confundimento do fatorial $2^{5}$ com uma única repetição, mostrando como se acha a es timativa do erro por intermédio das interaçóes de ordem elevada. Comentam a estimação do ganho em precisão com confundimento completo e parcial.

No fim do capitulo, apresentam värios planos de confundimento para os fatoriais das sēries $2^{n},: 3^{n}$ e mista.

Sobre os fatoriais fracionados, comentam o que acontece quando o experimento contém parte de uma repetí ção, exemp1ificando $\operatorname{com} 1 / 2$ da repetição do fatoria $12^{3}$. Apresentam uma discussão sobre $1 / 2$ do fatoria $12^{5}$ e do $2^{6}, 1 / 4$ do fatoria $1.2^{6}$ inteiramente ao acaso e $1 / 2$ do fatoria $12^{6}$ em blocos, dando os esquemas das respectivas anālises de variān cias. Apresentam uma adaptação do método prático de YATES aos fatoriais fracionados da série $2^{\text {n }}$. 
Sobre os fatoriais fracionados da série $3^{\text {n }}$, eles enfocam os problemas de construção desses experimentos através de $1 / 3$ da repetição do fatorial $3^{3}$. Apresentam värios planos no fim do capitulo, inclusive fazendo comentärios sobre $1 / 3$ do fatorial $3^{5}$ em blocos de 81,27 e 9 tratamentos.

KURKJIAN e ZELEN (1962), introduzem um mëtodo de cälculo para analisar os experimentos fatoriais no sentido geral, simétrico ou assimétrico. 0 objetivo principal desse método é realizar operações complexas, que são necessạ rias a estes experimentos, com relativa facilidade. 0 cälcu 10 possibilita muitas operações aritméticas extensas parcialmente realizadas por operaçoes lögicas. Isto seria de importância na programaça das anäliies dos fatoriais em com putadores.

os principais resultados desse trabalho são: (i) um desenvolvimento de uma nova teoria de confundimento para os fatoriais assimétricos, e (ii) uma nova maneira de ajuste para a regressão polinomial.

JOHN (1962), apresenta uma discussão sobre a construção e anālise dos delineamentos das séries $3\left(2^{n-p}\right)$.

ADELMAN (1963), apresenta uma revisão das téc nicas que selecionam as combinacões de tratamentos, que formam uma fração de um experimento fatorial. Apresenta värios 
procedimentos para a construção dos planos em fatorial fracionado. As técnicas discutidas incluem os planos ortogonal e não ortogonal para os experimentos fatoriais simétrico e assimétrico.. Os planos desenvolvidos vão desde aqueles que permitem somente a estimação dos efeitos principais, para aqueles que permitem a estimação dos efeitos principais e das interações de 2 fatores.

KURKJIAN e ZELEN (1963), aplicam o cälculo de senvolvido no trabalho anterior (KURKJIAN e ZELEN, 1962) para esquemas fatoriais em värios delineamentos experimentais. Os delineamentos considerados são aqueles que tem uma matriz de incidência $N$ tal que $N^{\prime}$ satisfaz um certa relação estrutura1. Quando esta relação estrutural é satisfeita, $N^{\prime}$ é dita ter a propriedade (A). Delineamentos que possuem esta propriedade são os blocos ao acaso, blocos incompletos balan ceados, grupo de delineamentos divisiveis e delineamentos em blocos incompletos parcialmente balanceado tipo quadrado latino com duas classes associadas. A razão por 1 imitar o estudo a estas classes de delineamentos é que eles são particü larmente usados em experimentos fatoriais assimétricos.

Nesta nota BANERJEE e FEDERER (1966), apresen tam uma generalização dos resultados obtidos em trabalhos an teriores (BANERJEE e FEDERER, 1963 e 1964), onde mostram como ajustar a matriz de incidēncia ( $X)$, para obtermos as esti mativas dos efeitos, como funções lineares ortogonais das ob 
servações para qualquer fatorial fracionado irregular de $N$ tratamentos. 0 fatorial fracionado considerado anteriormente foi tal que, a matriz de delineamento (x) era de dimensoes $p \times p$, sendo $p$ efeitos estimados de p observaçós. 0 mé todo consiste em achar a matriz $X$ tal que a matriz de delineamento (X) e o vetor de observaçóes (Y) sejam aumentados, i.è.,$X_{1}=\left[X^{\prime} ! X^{\prime} \lambda\right]^{\prime}$ de dimensão $(p+m) x$ p e $Y_{1}=\left[Y^{\prime} ! Y^{\prime} \lambda\right]^{\prime}$ de dimensão $(p+m) x 1, \operatorname{com} p+m=N$ de tal maneira que [ $\mathrm{X}_{1}^{\prime} \mathrm{X}_{1}$ ] seja uma matriz diagonal. Esta generalização è no sen tido que a matriz de delineamento não necessite ser quadrada, mas de dimensão $\left(p+m_{1}\right) x p, p+m_{1}<N$, onde os $p$ efeitos são estimados de $p+m_{1}$ observações.

Alëm dessa generalização, os autores apresentam os seguintes resultados adicionais: (i) a relação estrutural entre o efeito de parametros e as observaçoes foram de duzidas, (ii) uma regra foi desenvolvida para compor o fatorial fracionado irregular com observações que são internamen te consistentes tornando possivel estimar os efeitos de parâ metro, e (iii) um procedimento desejảvel para planejar o fatorial fracionado, para obter uma máxima eficiência.

KSHIRSAGAR (1966), prova o oposto do resultado obtido por KURKJIAN e ZELEN (1963), que provaram que deli neamentos fatoriais possuindo uma estrutura particular na ma triz C (propriedade A) são fatorialmente balanceados. 
MULLER (1966), apresenta uma discussão do uso de pseudo-fatores na construcão de esquemas fatoriais balanceados. 0 método é desenvolvido para delineamentos $s \quad x \quad e$ depois generaliza para delineamentos $5 \times q_{1} \times q_{2} \times \ldots x \quad q_{n}$, onde $\underline{s}$ é necessariamente uma potência de um primo. Usualmente blocos de $s^{t}$ parcelas são exigidos. o procedimento é substituir cada fator após o primeiro, por um ou mais pseudo -fatores em $\underline{s}$ niveis, e formar um delineamento original que confunde $s^{T}$ parcelas em blocos de $s^{t}$ para um $T$ adequado. Uma correspondēncia arbiträria é então estabelecida entre as com binações de niveis dos pseudo-fatores e os niveis $q_{u}$ do :fator $Q_{u}$. Isto fornece um conjunto de blocos inicial, para o delineamento requerido, e então uma série de permutações introduz o balanceamento. A ênfase principal é colocada sobre os delineamentos com 2 fatores. 0 caso especial dos delinea mentos $s \times q, \operatorname{com} s>q$ blocos de $\underline{s}$ parcelas, está relaciona do ao uso dos delineamentos em blocos incompletos balanceados na construção dos delineamentos fatoriais assimétricos.A relação parcialmente balanceada como forma de potência dois é também discutida. Métodos para reduzir o número dos primeiros blocos são examinados, como também são examinados os efeitos de escolha de correspondência dos niveis de fator e escolha das permutações no nümero de repetições.

Para os experimentos fatoriais, KEMPTHORNE (1975), inicia falando nos experimentos que envolvem värios fatores, e em seguida dos delineamentos fatoriais $2^{2}$ e $2^{3}$, e 
generaliza para a série $2^{n}$. Para os fatoriais da série $3^{n}$, faz uma discussão anäloga atrakés dos fatoriais $3^{2}$ e $3^{3}$.

Para confundimento, o autor apresenta o fatorial $2^{3}$ em blocos com 4 tratamentos. Apresenta um resumo em uma tabela dos tipos possiveis de confundimento para a série $2^{\mathrm{n}}$

No caso dos fatores da série $3^{n}$, apresenta o fatoria $13^{2}$ em blocos de 3 tratamentos utilizando 4 repetições, o fatorial $3^{3}$ em blocos de 3 tratamentos, é também o fatorial $3^{4}$ em blocos de 27,9 e 3 tratamentos.

Sobre os fatoriais fracionados, o autor os in troduz atravès de $1 / 2$ da repetição do fatorial $2^{3}$. Em segui da, apresenta $1 / 2$ do fatorial $2^{6}$ em blocos de 16 e 8 parcelas, $1 / 4$ do fatorial $2^{5}$ e $2^{8}$ inteiramente ao acaso e $1 / 4$ do fatorial $2^{8}$ em blocos de 16 e 8 parcelas. Também apresenta uma relação de identidade para $1 / 8$ do fatorial $2^{10}$ em blocos de 32 tratamentos.

Para os fatoriais da série $3^{n}$, apresenta uma discussão tomando como base $1 / 3$ do fatorial $3^{4}$ e $1 / 3$ do fato rial $3^{5}$ em blocos com 9 e 27 tratamentos.

ZONTA (1980), estabelece um processo de confundimento para os fatoriais da série $2^{n}$ e, através de duas propriedades deste, torna possivel o confundimento de fatoriais com qualquer número de niveis e de fatores. 
Apresenta para cada experimento fatorial da série $2^{n}$, conjuntos balanceados até o fatorial $2^{5}$, com a sub divisão das repetições em dois blocos, até a formação de blo cos de dois tratamentos, sem o confundimento de efeitos prin cipais.

Para os fatoriais da série $3^{n}$, apresenta conjuntos balanceados dos seguintes fatoriais: $3^{2}$ em blocos de 3 tratamentos, $3^{3}$ em blocos de 9 e 3 tratamentos e $3^{4}$ em blo $\cos$ de 37,9 e 3 tratamentos.

Para as séries mistas, apresenta um estudo dos fatoriais das seguintes séries: $3 \times 2^{n}, 3^{2} \times 2^{n}$ e $3^{3} \times 2^{n}$.

Além do método de confundimento, ele apresenta o processo de obtenção da informação relativa através da teoria dos blocos incompletos e o método geral da anälise de variāncia.

BIASI (1985), apresenta os elementos para se obter a anảlise de um experimento fatorial, em que é feita uma redução no número de parcelas experimentais. Também apre senta um desenvolvimento teörico sobre experimentos fatoriais, confundimento em experimentos fatoriais, frações' de fatoriais e teoria de Galois. Essas noções são necessārias para a apresentação de uma sistematização de alguns delineamentos apropriados para estudar efeitos principais.

Ela mostra como confundir efeitos principais 
e interações com blocos nos fatoriais, e depois como confundir interações de ordem maior ou igual a dois, de forma conveniente. Esse confundimento ë garantido pela existēncia de um teorema de FISHER. Por esse teorema toda interação na re lação de identidade tem no mínimo três letras.

São apresentados também os planos onde não ë possivel estabelecer uma relação de identidade, mas estimam efeitos principais.

Faz uma comparação entre os métodos destacando-se as vantagens e desvantagens, bem como a adequação de cada um. E também indica outras formas de delineamentos experimentais que poderiam ser usados como planos de efeitos principais.

LIMA (1986), apresenta um estudo sobre os experimentos de resolução V. Mostra também como obter geradores tais, que os efeitos principais e interaçóes de 2 fatores não sejam confundidos.

Obtēm esses geradores sem nenhum método específico, ou seja, através de tentativas, observando o porquê de alguns autores denominarem tais experimentos de planos de 5 letras. Obtido. os geradores, apresenta os tipos possiveis de experimentos por eles gerados. Isto é feito através de exemplos para o fatorial da série $2^{n}, n=3,4, \ldots, 8$ Em seguida, apresenta um método do qual obtemos diretamente tais 
geradores, sendo exemplificado através dos fatores $2^{\mathrm{n}}, \mathrm{n}=8$, 9, 10, 11. Apresenta para cada caso, uma relação de identidade para cada uma das fraçoes de fatoriais possiveis e o quadro da anālise de variāncia correspondente. Mostra como obter experimentos de resolução V em blocos casualizados, e finalmente, apresenta um exemplo numérico utilizando a fração do fatorial $2_{\mathrm{V}}^{6-1}$.

Para os esquemas fatoriais da série $3^{\text {n }}$, apresenta uma discussão anāloga aos fatoriais da série $2^{\text {n }}$, utili zando os fatoriais $3^{5}$ e $3^{6}$.

Quanto aos fatoriais da série $4^{\text {n }}$ apresenta apenas como os corpos de Galois são tratados para a obtenção de experimentos de resolução $V$.

No final, faz observações sobre a utilização do fatorial fracionado, e alerta aos pesquisadores que devem tomar cuidados entre outros, na escolha dos geradores e oonhecimento dos aliados dos efeitos fatoriais. 


\section{MÉTODO E MATERIAL}

\subsection{MÉTOdO}

\subsubsection{EXPERIMENTOS FATORIAIS}

os ensaios fatoriais são aqueles em que os tratamentos resultam de todas as combinaçós possiveis dos niveis dos diversos fatores.

Uma grande vantagem dos ensaios fatoriais ë que eles nos permitem tirar conclusões mais amplas a respeito dos fatores (qualitativos ou quantitativos) que estão sen do estudados, uma vez que são pesquisados simultaneamente di ferentes combinacões de seus niveis.

Estes esquemas não constituem um delineamento experimental e, sim, um delineamento de tratamentos, podendo 
assim ser colocado nos mais diferentes delineamentos experimentais.

\section{1 .1 .1 . EXPERIMENTOS FATORIAIS DA SÉRIE $2^{n}$}

\subsection{Modelo Matemātico}

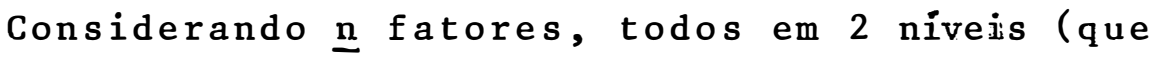
podem ser considerados superior e inferior, ou ausència e pre sença), temos o mode1o matemätico no sentido gera1;

$$
\mathrm{Y}_{i j k: \ldots}=\mu+\alpha_{i}+\beta_{j}+(\alpha \beta)_{i j}+\delta_{k}+(\alpha \delta)_{i k}+(\beta \delta)_{j k}+\ldots+e_{i j k} \ldots
$$

onde

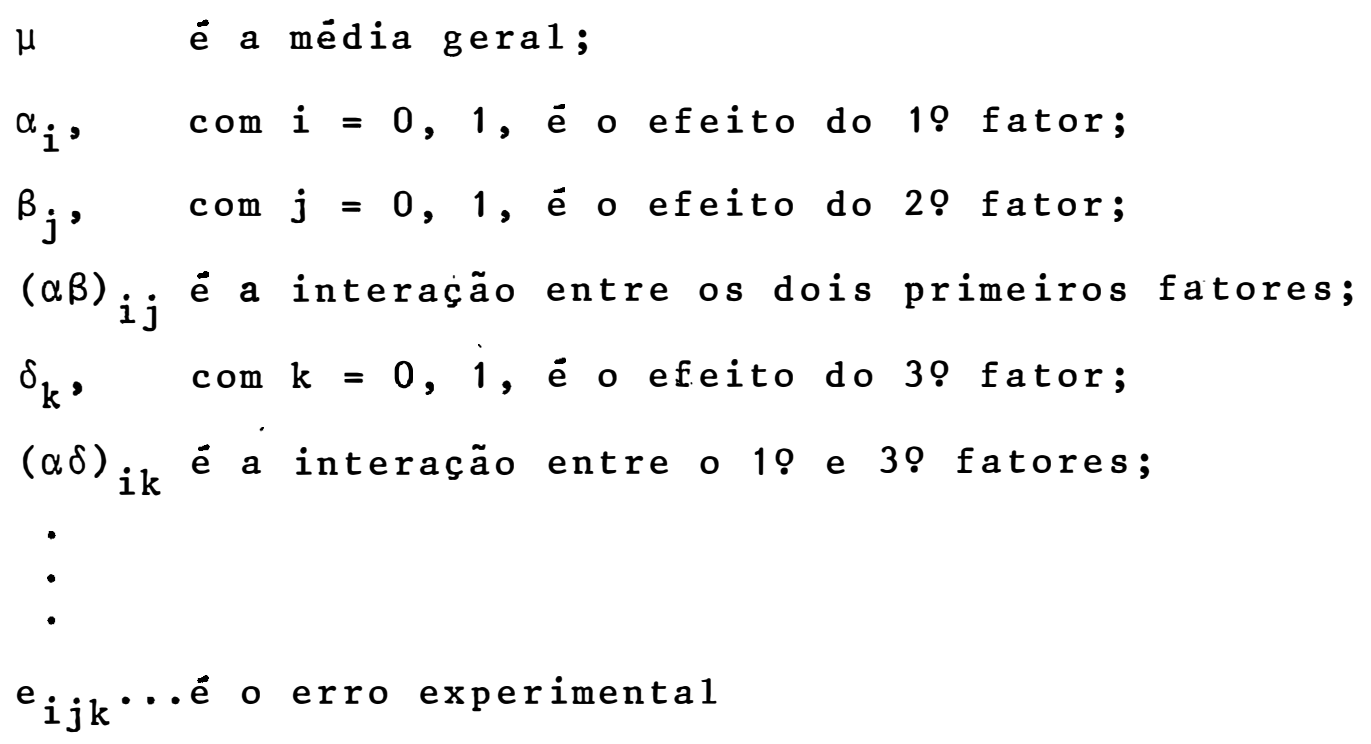
mar como exemplo o fatorial com 2 fatores em 2 niveis. Assim as combinações de tratamentos são: (1), a, b, ab, onde: 
(1): indica que os 2 fatores estão no nivel inferior;

a : indica que o fator A estä no nivel superior e o fator B està no nível inferior;

b : indica que o fator A estä no nivel inferior e o fator B estä no nivel superior;

ab : indica que os fatores $A$ e $B$ estão no nivel superior.

A notação (1), a, b, ab tambëm serà usada para indicar a média das observações tomadas nas respectivas combinações de tratamentos.

Os efeitos principais e a interação são calcú ladas com base na Tabela 1 .

TABELA 1. Os efeitos principais e interação.

\begin{tabular}{|c|c|c|c|c|}
\hline \multirow{2}{*}{ Efeitos } & \multicolumn{4}{|c|}{ Tratamentos } \\
\hline & (1) & $\mathrm{a}$ & $b$ & $a b$ \\
\hline 2. $\mathrm{A}$ & - & + & - & + \\
\hline $2 B$ & - & - & + & + \\
\hline $2 \mathrm{AB}$ & + & - & - & + \\
\hline
\end{tabular}

Assim, como vemos, os contrastes são:

$$
\begin{aligned}
& A=a b+a-b-(1)=(a-1)(b+1) \\
& B=a b+b-a-(1)=(a+1)(b-1) \\
& A B=a b+(1)-a-b=(a-1)(b-1)
\end{aligned}
$$


Notamos que os 3 contrastes acima são mutua mente ortogonais.

De uma forma geral, nös podemos representar os efeitos da seguinte maneira:

$$
x=\frac{1}{r 2^{n-1}}[(a \pm 1)(b \pm 1)(c \pm 1) \cdots] \text {, onde }
$$

r é o nümero de repetições de cada combinação de tratamento, e o sinal entre parenteses é positivo se a letra maiúscula correspondente não está contida em $x$ e negativo se ela está contida em $\mathrm{X}$.

Para exemplificar, vamor tomar o fatorial $2^{6}$, com os fatores A, B, C, D, E e F, todos em 2 niveis, e então a interação $A B C$ pode ser assim representada:

$$
A B C=\frac{1}{32 r}[(a-1)(b-1)(c-1)(d+1)(e+1)(f+1)]
$$

\subsection{Forma geral de apresentar os fato- riais $2^{n}$}

Nesse sistema são testadas todas as combinações de $n$ fatores cada um em dois níveis. A totalidade das combinações de tratamentos podem ser representadas geometricamente num sistema de coordenadas n-dimensional de comprimen

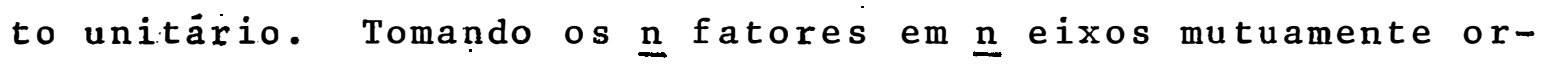


togonais $x_{1}, x_{2}, \ldots, x_{n}$, teremos o ponto $(0,0, \ldots, 0)$ como sendo o tratamento controle; $(1,0,0, \ldots, 0)$ o primeiro fator no nivel superior e todos os outros no nivel mais baixo, e assim por diante.

Por exemp1o, o fatorial $2^{2}$, com os fatores A e $B$ representados respectivamente, nos eixos $x_{1}$ e $x_{2}$.

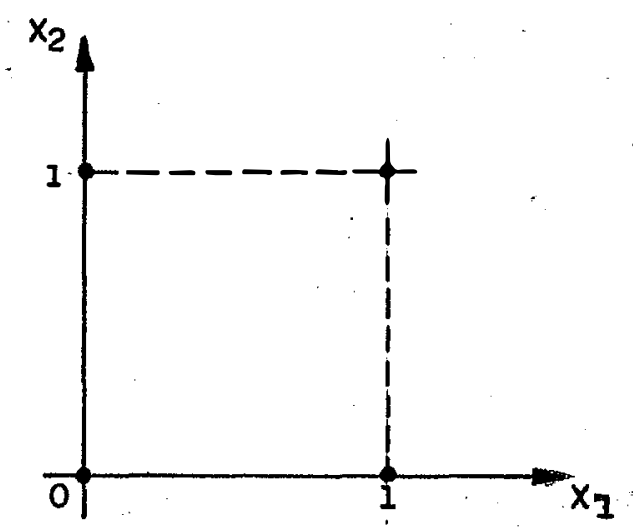

Assim, as 4 combinações de tratamentos podem ser representadas pelos pontos $(0,0) ;(1,0) ;(0,1)$ e $(1,1)$.

Atravēs da notação acima, vemos que o efeito de $A$ è a diferença entre as mẻdias dos pontos representados por $x_{1}=0$ e $x_{1}=1$. Da mesma forma, o efeito de $B$ é a diferença entre as médias dos pontos representados por $x_{2}=0$ e $x_{2}=1$.

Como vemos, a interação $A B$ è a diferença entre as médias dos pontos $(0,0)$ e $(1,1)$, e as mëdias dos pontos $(1,0)$ e $(0,1)$. Tomando a soma das variäveis $x_{1}+X_{2}$ para os pontos usados na interação temos: 


$$
\begin{aligned}
& \text { Para o ponto }(0,0), x_{1}+x_{2}=0 \\
& \text { Para o ponto }(1,1), x_{1}+x_{2}=2 \\
& \text { Para o ponto }(1,0), x_{1}+x_{2}=1 \\
& \text { Para o ponto }(0,1), x_{1}+x_{2}=1
\end{aligned}
$$

Se nös estamos trabalhando através da geometria finita com nümeros reduzidos a módulo 2 para essas somas, i.è, nós substituímos qualquer nümero maior do que 1 (hum) pelo resto após ele ser dividido por 2 , então a intera ção $A B$ serä a diferença média entre os pontos representados por:

$$
\begin{aligned}
& x_{1}+x_{2}=0(\bmod \cdot 2) \\
& x_{1}+x_{2}=1(\bmod \cdot 2)
\end{aligned}
$$

Da mesma maneira podemos raciocinar em termos. de geometria finita para os efeitos principais. Assim, o efeito de A seria obtido através da diferença dos $X_{1}=0$ $(\bmod \cdot 2) \operatorname{com} x_{1}=1(\bmod \cdot 2)$, ou seja:

Para o ponto $(0,0), x_{1}=0$, reduzindo o modulo 2 temos

$$
x_{1}=0(\bmod \cdot 2)
$$

Para o ponto $(0,1), x_{1}=0$, reduzindo o modulo 2 temos

$$
\mathrm{X}_{1}=0(\bmod \cdot 2)
$$

Para o ponto $(1,0), X_{1}=1$, reduzindo o modulo 2 temos

$$
X_{1}=1:(\bmod \cdot 12)
$$

Para o ponto $(1,1), X_{1}=1$, reduzindo o modulo 2 temos

$$
x_{1}=1(\bmod \cdot 2)
$$


Para o efeito de B procede-se da mesma forma.

Agora vamos tomar como exemplo:o fatorial $2^{3}$, com os fatores A, B e C representados respectivamente, nos. eixos $x_{1}, x_{2}$ e $x_{3}$.

Na nossa notação, podemos representar as combinações de tratamentos por um ponto em 3 dimensões,ou seja:

$$
\begin{aligned}
& 1=(0,0,0) ; a=(1,0,0) ; b=(0,1,0) ; a b=(1,1,0) ; c=(0,0,1) \\
& c=(1,0,1) ; b c=(0,1,1) ; a b c=(1,1,1)
\end{aligned}
$$

A representação gräfica será:

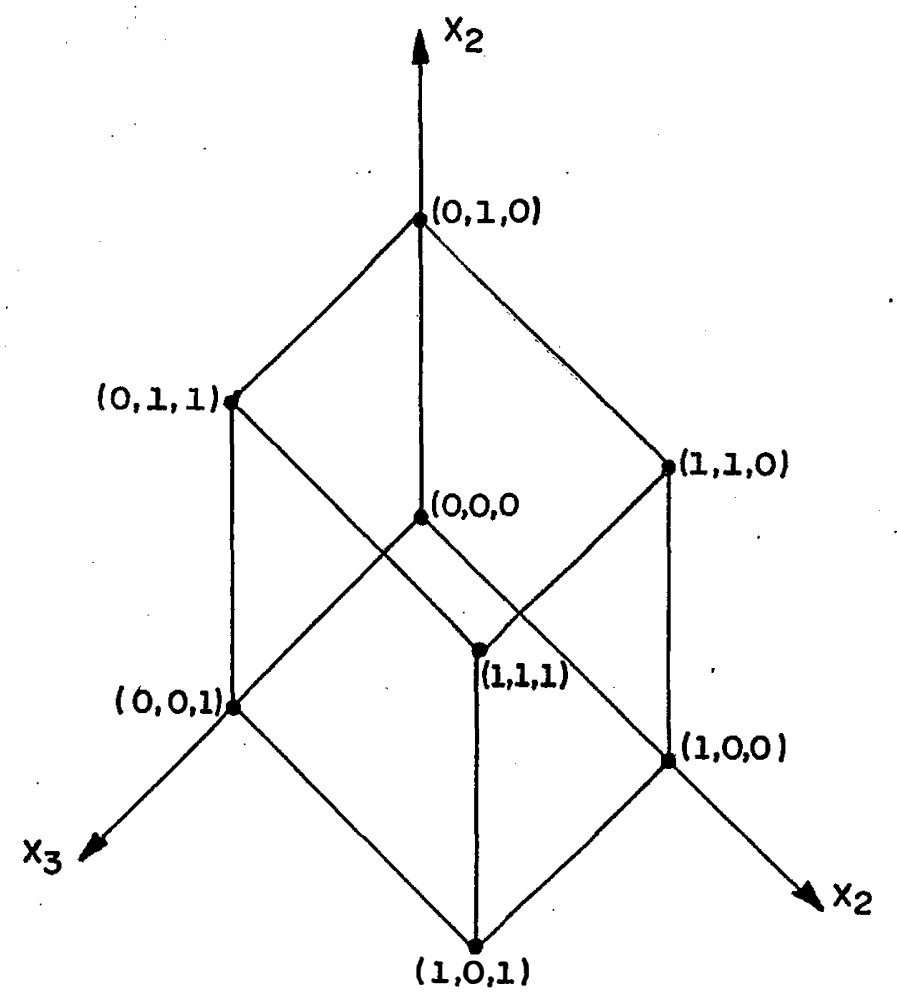

0 efeitodo fator A, é a diferença das médias dos pontos que contēm $x_{1}$ com os que não contém ou seja, é a diferença entre a mëdia dos pontos que estão no plano $x_{1}=1$ 
e a média daqueles que estão no plano $x_{1}=0$. 0 mesmo raciocí nio vale para os efeitos dos fatores B e C. A interação de A e $B$ é a diferença entre as médias dos pontos representados por $x_{1}=1, x_{2}=1$ e $x_{1}=0, x_{2}=0$, e aqueles representados por $x_{1}=0, x_{2}=1$ e $x_{1}=1, x_{2}=0$.

Também podemos escrever os efeitos principais e as interações como equações algébricas, cujas operações obedecem a aritmética modular, então temos:

FATOR $A: x_{1}=0(\bmod , 2)$ contra $x_{1}=1(\bmod \cdot 2)$

FATOR B : $\mathrm{x}_{2}=0(\bmod \cdot 2)$ contra $x_{2}=1(\bmod \cdot 2)$

FATOR $C: \mathrm{X}_{3}=0(\bmod \cdot 2)$ contra $\mathrm{x}_{3}=1(\bmod \cdot 2)$

INTERAÇAO A $\mathrm{x}$ B : $\mathrm{x}_{1}+\mathrm{x}_{2}=0(\bmod \cdot 2)$ contra $\mathrm{x}_{1}+\mathrm{x}_{2}=1 \quad(\bmod .2)$

INTERAÇAO A $\mathrm{x} C: \mathrm{x}_{1}+\mathrm{x}_{3}=0(\bmod , 2)$ contra $\mathrm{x}_{1}+\mathrm{x}_{3}=1 \quad(\bmod .2)$

INTERAÇAO B $\times$ C : $\mathrm{X}_{2}+\mathrm{X}_{3}=0 \quad(\bmod \cdot 2)$ contra $\mathrm{X}_{2}+\mathrm{X}_{3}=1 \quad(\bmod .2)$ INTERAÇAO A $\quad x \quad$ B $x \quad C \quad: x_{1}+x_{2}+x_{3}=0(\bmod .2)$ contra

$$
\mathrm{x}_{1}+\mathrm{x}_{2}+\mathrm{x}_{3}=1(\bmod \cdot 2)
$$

Assim, podemos generalizar para o fatorial $2^{n}$, onde temos a diferença entre as médias dos pontos representa dos por:

$$
x_{1}+x_{2}+\cdots+x_{n}=0(\bmod \cdot 2)
$$

e

$$
x_{1}+x_{2}+\cdots+x_{n}=1(\bmod \cdot 2)
$$




\subsection{A anālise de variāncia para os fa- toriais da série $2^{n}$}

Tomando o experimento fatorial $2^{\mathrm{n}}$ inteiramente ao acaso com $\underline{r}$ repetições, a anālise de variāncia será:

\begin{tabular}{|c|c|}
\hline C. variação & G.L. \\
\hline Tratamentos & $2^{n}-1$ \\
\hline Efeitos principais & $\left(\begin{array}{c}\mathrm{n} \\
1\end{array}\right)$ 1inhas com $1 \mathrm{~g} \cdot 1$. \\
\hline Interações de 2 fatores & $\left(\begin{array}{l}\mathrm{n} \\
2\end{array}\right)$ 1 inhas com $1 \mathrm{~g} \cdot 1$. \\
\hline : & $\begin{array}{ll}\cdot & . \\
\cdot & .\end{array}$ \\
\hline Interações de n fatores & $\left(\begin{array}{l}n \\
n\end{array}\right)$ inhas com $1 \mathrm{~g} \cdot 1$. \\
\hline Residuo & $2^{n}(r-1)$ \\
\hline Tota 1 & $r 2^{n}-1$ \\
\hline
\end{tabular}

Para calcular os estimadores dos efeitos prin cipais e interações, e suas somas de quadrados correspondentes, usaremos o procedimento sistemático de YATES, que será descrito mais tarde no exemplo numérico. 


\subsubsection{EXPERIMENTOS FATORIAIS DA SÉRIE 3"}

\subsection{Modelo Matemātico}

Considerando $\underline{\mathrm{n}}$ fatores, todos em 3 niveis, i. é., $3^{n}$. Vamos representar os 3 niveis de cada fator por 0,1 e 2. 0 modelo matemático no sentido geral será:

$$
\mathrm{Y}_{i j k} \ldots=\mu+\alpha_{i}+\beta_{j}+(\alpha \beta)_{i j}+\delta_{k}+(\alpha \delta)_{i k}+(\beta \delta)_{j k}+\ldots+e_{i j k} \cdots
$$

onde :

$\mu \quad$ è a mèdia geral;

$\alpha_{i}, \quad \operatorname{com} i=0,1,2$ é o efeito do 1 ị fator;

$\beta_{j}, \quad \operatorname{com} j=0,1,2$ é o efeito do 20 fator;

$(\alpha \beta)_{i j}$ é a interação entre os dois primeiros fatores;

$\delta_{k}, \quad \operatorname{com} k=0,1,2$ é o efeito do 39 fator;

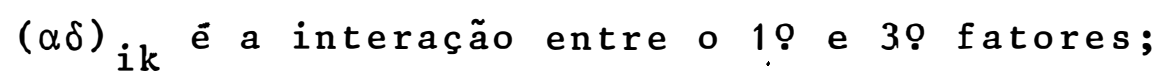

$e_{i j k} \quad \dot{e}$ o erro experimental.

\subsection{A forma geral de apresentar os fa- toriais $3^{\text {n }}$}

Nesse esquema são testadas todas as combina-. çoes de $\underline{n}$ fatores, cada um em 3 níveis. As combinações de tratamentos podem ser representadas geometricamente num sis- 
tema de coordenadas n-dimensionais contendo em cada eixo três pontos. Tomando os $\underline{\mathrm{n}}$ eixos $\left(\mathrm{x}_{1}, \mathrm{x}_{2}, \ldots, \mathrm{x}_{\mathrm{n}}\right)$ mutuamente ortogonais, teremos o ponto $(0,0, \ldots, 0)$ como sendo o tratamento controle; $(1,0, \ldots, 0)$ como sendo o primeiro fator no. segundo nivel e os outros fatores ao nivel mais baixo; $(2,0, \therefore \ldots, 0)$ como sendo o primeiro fator no nivel mais alto e os outros no nive1 mais baixo, e assim por diante.

Tomando por exemplo, o fatorial $3^{2}$, com os tores $A$ e B representados respectivamente, nos eixos $X_{1}$ e $x_{2}$, temos :

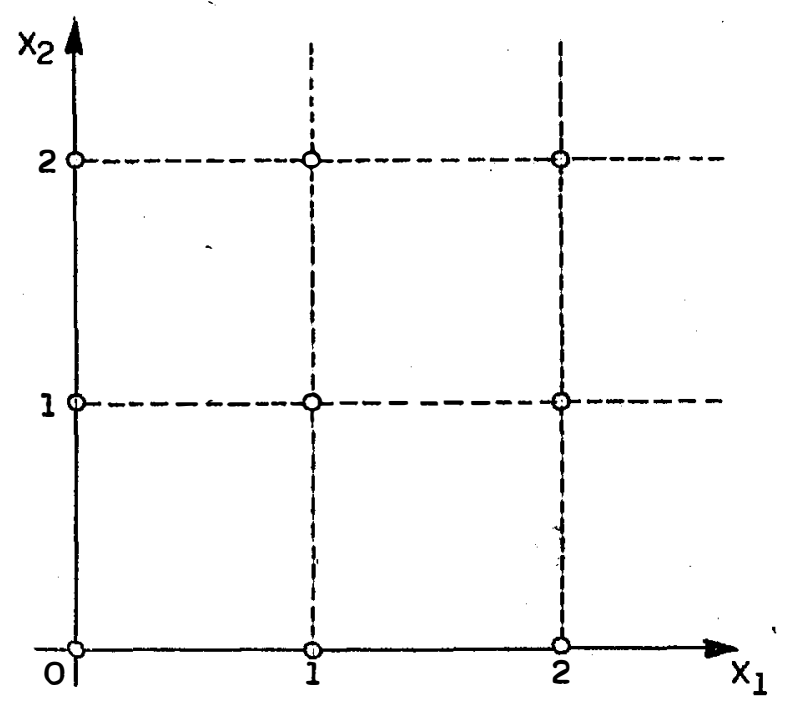

Assim, as 9 combinações de tratamentos podem ser representadas pelos pontos:

$$
\begin{aligned}
& (0,0) ;(1,0) ;(2,0) \\
& (0,1) ;(1,1) ;(2,1) \\
& (0,2) ;(1,2) ;(2,2) .
\end{aligned}
$$

os fatores principais e as interacões para o fatorial $3^{2}$ através da geometria finita tomando-se modulo 3 
(substituir qualquer nümero maior do que 2 (dois) pelo resto após ele ser dividido por 3 ) podem ser representados em modu 103 como segue :

FATOR A:

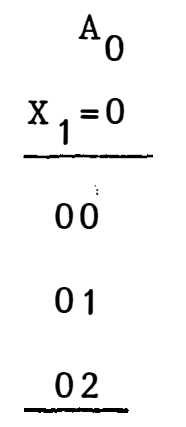

$\begin{array}{r}A_{1} \\ \mathrm{X}_{1}=1 \\ \hline 10 \\ 11 \\ 12 \\ \hline\end{array}$

$\mathrm{A}_{2}$

\begin{tabular}{c}
$\mathrm{x}_{1}=0$ \\
\hline 00 \\
01 \\
02 \\
\hline
\end{tabular}

FATOR B :

$\begin{array}{r}{ }^{\mathrm{B}} 0 \\ \mathrm{X}_{2}=0 \\ \hline 00 \\ 10 \\ 20 \\ \hline\end{array}$

$\mathrm{B}_{1}$

$$
\frac{\mathrm{x}_{2}=1}{01}
$$

11

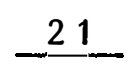

INTERAÇAO AB OU $A^{2} B^{2}$ :

$$
\begin{gathered}
\mathrm{AB}_{0}: \\
\mathrm{x}_{1}+\mathrm{X}_{2}=0 \\
\hline 00
\end{gathered}
$$

12

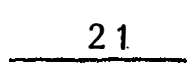

INTERAÇAO $\mathrm{AB}^{2}$ ou $\mathrm{A}^{2} \mathrm{~B}$ :

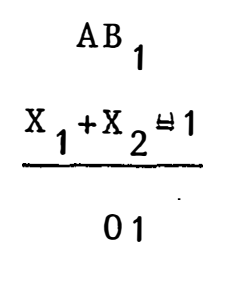

10

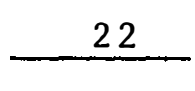

$\mathrm{AB}_{1} 2$

$$
\frac{x_{1}+2 x_{2}=1}{02} \frac{x_{1}+2 x_{2}=2}{01}
$$

10

12
$\mathrm{AB}_{0}^{2}$

$$
\frac{x_{1}+2 x_{2}=0}{00}
$$

11

22

\begin{tabular}{rr}
10 & 12 \\
21 & 20 \\
\hline
\end{tabular}


Pe1o exposto acima, vemos que os fatores A e B tem 2 graus de 1 iberdade cada um, pois os mesmos são dados. pelos contrastes entre os 3 grupos de combinações de tratamentos.

A interação entre os fatores $A$ e $B$ é a intera ção de uma tabela 3 x 3 , e assim temos $(3-1)(3-1)=4$ graus de 1 iberdade.

Assim, os 2 graus de 1 iberdade :correspondente. a $A B$ são dados pelos contrastes entre os 3 grupos de combinações de tratamentos seguintes:

$$
\left.\begin{array}{l}
x_{1}+x_{2}=0 \\
x_{1}+x_{2}=1 \\
x_{1}+x_{2}=2
\end{array}\right\}(\bmod 3)
$$

e estas equações são idēnticas com aquelas que especificam os 3 grupos de combinações de tratamentos denotado por $\mathrm{A}^{2} \mathrm{~B}^{2}$, ou seja,

$$
\begin{aligned}
& 2\left(\mathrm{x}_{1}+\mathrm{x}_{2}\right)=2 \mathrm{x} 0 \Longrightarrow 2 \mathrm{x}_{1}+2 \mathrm{x}_{2}=0 \\
& \left.2\left(\mathrm{x}_{1}+\mathrm{x}_{2}\right)=2 \mathrm{x} 1 \Longrightarrow 2 \mathrm{x}_{1}+2 \mathrm{x}_{2}=2\right\}(\bmod \cdot 3) \\
& 2\left(x_{1}+x_{2}\right)=2 \times 2 \Longrightarrow 2 x_{1}+2 x_{2}=1
\end{aligned}
$$

Sinilarmente, os 2 graus de 1 iberdade correspondente a $\mathrm{AB}^{2}$ são os mesmos graus de 1 iberdade que correspondem a $A^{2} B$, ou seja: 


$$
\left.\begin{array}{rl}
A B^{2}: x_{1}+2 x_{2} & =0 \\
x_{1}+2 x_{2} & =1 \\
x_{1}+2 x_{2} & =2
\end{array}\right\} \quad(\bmod 3)
$$

Então:

$2\left(x_{1}+2 x_{2}\right)=2 x \cdot 0 \Longrightarrow 2 x_{1}+4 x_{2}=0$, tomando-se modulo 3 , temos

$$
2 \mathrm{x}_{1}+\mathrm{x}_{2}=0
$$

$2\left(x_{1}+2 x_{2}\right)=2 \times 1 \Longrightarrow 2 x_{1}+4 x_{2}=2$, tomando-se modulo 3 , temos

$$
2 \mathrm{x}_{1}+\mathrm{x}_{2}=2
$$

$2\left(x_{1}+2 x_{2}\right)=2 \times 2 .>2 x_{1}+4 x_{2}=4$, tomando-se modulo 3 , temos

$$
2 x_{1}+x_{2}=1
$$

Vemos que os grupos dados pelos simbolos AB e $A^{2} B^{2}$, e $A B^{2}$ e $A^{2} B$ são os mesmos. Portanto, convenciona.- se que para obter uma enumeraça completa e ünica dos graus de liberdade, adotamos a regra que a potēncia da primeira letra de uma interação seja sempre igual a 1 (hum).

Agora, tomando como exemplo, o fatorial $3^{3}$, com os fatores $A, B, e$, temos os resultados mostrado abaixo: 
Fatores principais e interações

Equação

$\left.\begin{array}{ll}A & x_{1} \\ A B & x_{2} \\ A B^{2} & x_{1}+x_{2} \\ C & x_{1}+2 x_{2} \\ A C & x_{3} \\ A C^{2} & x_{1}+x_{3} \\ B C & x_{1}+2 x_{3} \\ \mathrm{BC}^{2} & x_{2}+x_{3} \\ A B C^{2} & x_{2}+2 x_{3} \\ A B^{2} C & x_{1}+x_{2}+x_{3} \\ A B^{2} C^{2} & x_{1}+x_{2}+2 x_{3} \\ A B C & x_{1}+2 x_{2}+x_{3} \\ A & x_{1}+2 x_{2}+2 x_{3}\end{array}\right\}=0,1,2$

Deve ser observado que os 8 graus de liberdade para a interação entre os 3 fatores são subdivididas em 4 grupos de 2 graus de 1 iberdade cada um.

Para ofatorial com 4 fatores, todos em 3 niveis, serão apresentadas apenas as equacões que correspondem as interações de 4 fatores. Temos 8 grupos com 2 graus de liberdade cada, que são mostrados abaixo: 
Interações de 4 fatores
$A B C D$
$A B C D^{2}$
$A B C^{2} D$
$A B C^{2} D^{2}$
$A B^{2} C D$
$A B^{2} C D^{2}$
$A B^{2} C^{2} D$
$A B^{2} C^{2} D^{2}$

Equação

$$
\left.\begin{array}{l}
x_{1}+x_{2}+x_{3}+x_{4} \\
x_{1}+x_{2}+x_{3}+2 x_{4} \\
x_{1}+x_{2}+2 x_{3}+x_{4} \\
x_{1}+x_{2}+2 x_{3}+2 x_{4} \\
x_{1}+2 x_{2}+x_{3}+x_{4} \\
x_{1}+2 x_{2}+x_{3}+2 x_{4} \\
x_{1}+2 x_{2}+2 x_{3}+x_{4} \\
x_{1}+2 x_{2}+2 x_{3}+2 x_{4}
\end{array}\right\}
$$

\subsection{Anāiise de variància para o fato- rial da sērie $3^{n}$}

Tomando o experimento fatorial $3^{\mathrm{n}}$ inteiramente ao acaso com $\underline{r}$ repetições, a anảlise de variância será:

C. Variação

Tratamentos

Efeitos principais

Interações de 2 fatores

Interação :de $n$ fatores

Residuo

Tot a 1

$$
r 3^{n}-1
$$

G.L .

$$
3^{n}-1
$$

$\left(\begin{array}{c}\text { n } \\ 1\end{array}\right)$ linhas com 2 g. 1 .

$\left(\begin{array}{l}n \\ 2\end{array}\right)$ I inhas com $4 \mathrm{~g} \cdot 1$.

$$
1 \text { inhas } \operatorname{com} 2^{n} g \cdot 1 \text {. }
$$$$
3^{n}(r-1)
$$ 
Para calcular os estimadores dos efeitos prin cipais e interações, e suas respectivas somas de quadrados, utilizaremos a geometria finita, que será apresentada mais tarde no exemplo numérico.

\subsubsection{CONFUNDIMENTO NOS EXPERIMENTOS FATORIAIS}

Como vimos no item 2, uma das desvantagens

dos esquemas fatoriais, é que o número de tratamentos cresce rapidamente na medida que o número de fatores aumenta, e com isto traz uma série de problemas para o pesquisador, dentre eles a perda de eficiência, pois a medida que aumenta o tama nho do bloco, aumenta a heterogeneidade dentro dele.

Uma das maneiras de contornar este problema é o uso da técnica do confundimento, que consiste em confundir interações de ordem elevada com efeito de blocos, dividindo, cada repetição em um número maior de blocos, perdendo toda ou parte da informação sobre as interações confundidas.

o confundimento é empregado quando não há con dições de instalar todas as combinaçoes de tratamento numa determinada repetição e também não hả muito interesse em um determinado efeito, que vai ser confundido. 


\section{1 .2 .1 . CONFUNDIMENTO NA SÉRIE $2^{n}$}

Nos experimentos fatoriais $2^{n}$, confundir uma interação consiste em dividir as combinações de tratamento de uma repetição em dois conjuntos ou blocos, tal que a diferen ça entre os conjuntos ou blocos represente a interação confundida. Quando mais de uma interação é confundida, as combinações de tratamentos de uma repetição serão divididas em $4,8,16, \ldots$ conjuntos ou blocos, de acordo com o número de interações confudidas.

FINNEY (1945), apresenta as seguintes regras para o confundimento na série $2^{\text {n }}$.

i) Todas as combinações de tratamentos no bloco principal (aquele que contém o tratamento controle, que é representado por (1)), contém um nümero par de letras em comum com a interação confundida.

Por exemplo, se num experimento fatorial $2^{4}$, a interação $A B C$ é confundida, o bloco principal consiste de 8, combina ções de tratamentos que contém um número par de letras em comum com a interação $A B C$, considerando zero como um numero par, então o bloco principal serä:

(1), d, ab, abd, ac, acd, bc, bcd.

i i) o produto de duas combinações de tratamentos pertencente ao bloco principal, continua pertencendo a este bloco. 0 produto de dois símbolos (quaisquer combinações de tra 
tamentos ou efeitos) è o mesmo como aquele dado na algebra comum, exceto que qualquer letra que ocorre duas vezes é cancelada. Por exemplo,

$$
\begin{aligned}
& a b \times d=a b d \\
& A B C \times A C D=A^{2} B^{2} D=B D
\end{aligned}
$$

iii) o produto de dois contrastes confundido, também serä confundido, e será chamado de interação generalizada. No exemplo acima, se o pesquisador escolheu para confun dir $A B C$ e $A C D$, então ele também está confundindo $B D$, i.è.,

$$
A B C \times A C D=A^{2} B C^{2} D=B D
$$

iv) Se todas as combinações de tratamentos do bloco principal são multiplicadas por uma combinação de tratamento não pertendente a este bloco, as combinações de tratamen tos obtidas formam um outro bloco do mesmo esquema de confundimento.

\section{EXEMPLO 1 .}

Vamos tomar o experimento fatorial $2^{3}$ :com uma repetição, confundindo a interação $A B C$. As oito combinações de tratamentos são: (1), a, b, ab, c, ac, bc e abc.

Pela regra (i), temos que o bloco principal coñtẻm 4 combinações de tratamentos com um número par de letras em comum com a interação confundida $A B C, i . e ̀,(1)$; $a b$, 
ac e bc. Assim temos os seguintes blocos:

$\begin{array}{rl}\text { BLOCO I } & \text { BLOCO II } \\ (1)=000 & a=100 \\ \mathrm{ab}=110 & \mathrm{~b}=010 \\ \mathrm{ac}=101 & \mathrm{c}=001 \\ \mathrm{bc}=011 & \text { abc }=111\end{array}$

Sabemos também que o contraste $A B C$ é definido

por:

$$
A B C=a+b+c+a b c-(1)-a b-a c-b c
$$

Assim, vemos que confundindo o contraste $A B C$, o bloco I ou bloco principal, é constituido pelas combinações de tratamentos que levam o sinal - então serä definido por $I=-A B C$.

E o bloco II é constituido pelas combinações de tratamentos que levam o sina $1+$, então será definido por $I=+A B C$.

Em geral, para um efeito qualquer $X$, o conjun to de pontos que tem sinais positivos (ou negativos) no contraste $X$ é chamado de fração dos pontos $2^{n}$, definidos por: $I=+X$, ou $I=-X$.

o contraste escolhido para ser confundido é chamado de contraste de definição da forma de confundimento. 
Uma forma alternativa de apresentar as combinações de tratamentos para os fatoriais $2^{n}$, è associar os $\underline{n}$

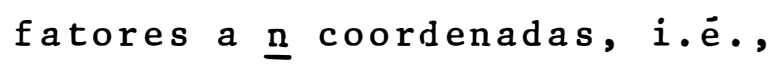

$\mathrm{x}_{\mathrm{i}}$ : tem o valor -1 quando os fatores estão todos no nível in ferior;

$\mathrm{x}_{\mathrm{i}}$ : tem o valor +1 quando os fatores estão todos no nivel su perior.

No nosso exemplo acima, associamos os fatores $A, B$ e C a trēs coordenadas $x_{1}, x_{2}$ e $x_{3}$. Assim temos:

$\mathrm{x}_{1}$ : tem o valor -1 quando A está no nivel inferior, e $\mathrm{x}_{1}$ : tem o valor +1 quando A está no nivel superior.

\section{Similarmente, temos que:}

$\mathrm{x}_{2}=-1$, se $B$ está no nivel inferior

$\mathrm{x}_{2}=+1$, se B está no nivel superior

e

$x_{3}=-1$, se C está no nivel inferior

$x_{3}=+1$, se C está no nivel superior

Assim, as 8 combinações de tratamentos podem ser representadas como segue:

$$
\begin{aligned}
& (1)=(-1,-1,-1) ; a=(1,-1,-1) ; b=(-1,1,-1) ; a b=(1,1,-1) ; \\
& c=(-1,-1,1) ; a c=(1,-1,1) ; b c=(-1,1,1) ; a b c=(1,1,1)
\end{aligned}
$$

Com base nessa notação, os blocos do, nosso 
exemplo 1 também podem ser representados da seguinte maneira:

o bloco I, serä representado pelo conjunto de pontos para o qual $x_{1} x_{2} x_{3}=-1$, se fizermos $x_{i}= \pm 1$, ou em termo de geometria finita pelo conjunto de pontos para: o qual $x_{1}+X_{2}+X_{3}=0(\bmod \cdot 2)$, se fizermos $x_{i}=0,1$.

o bloco II, serä representado pelo conjunto de pontos para o qual $x_{1} x_{2} x_{3}=+1$, se fizermos $x_{i}= \pm 1$, ou em termo de geometria finita pelo conjunto de pontos para oqual $x_{1}+x_{2}+X_{3}=1(\bmod \cdot 2)$, se fizermos $x_{i}=0,1$

A estrutura da anälise de variância para o ex perimento $2^{3}$ em dois blocos com 4 tratamentos, repetido re zes, confundindo a interação $A B C$ è: 


\begin{tabular}{lc}
\hline C. Variação & G.L. \\
\hline (Blocos) & $(2 \mathrm{r}-1)$ \\
Repetições & $\mathrm{r}-1$ \\
ABC & 1 \\
Repetições x ABC & $\mathrm{r}-1$ \\
(Tratamentos) & $(6)$ \\
A & 1 \\
B & 1 \\
AB & 1 \\
C & 1 \\
AC & 1 \\
BC & 1 \\
Residuo & $6(\mathrm{r}-1)$ \\
\hline Total & $8 \mathrm{r}-1$ \\
\hline
\end{tabular}

EXEMPLO 2.

Agora vamos tomar o experimento fatorial $2^{4}$ com uma repetição, confundindo as interações ABC e ACD. As 16 combinações de tratamentos são: (1), a, b, ab, c, ac, bc, $a b c, d, a d, b d, a b d, c d, a c d, b c d e a b c d$.

Pela regra (i), temos que o bloco principal contẻm as 4 combinações de tratamentos com um número par de letras em comum com as 2 interações confundidas ABC e ACD, 
i.è., (1), ac, abd e bcd, então temos:

$\underline{B L O C O I}$

(1)

ac

$a b d$

bcd

\section{BLOCO II}

a

c

bd

abcd
BLOCO III

b

$a b c$

ad

cd $\underline{\text { BLOCO IV }}$

d

acd

$a b$

b c

onde,

BĹaco II: é obtido por multiplicar as combinações de tratamentos de bloco I por

BLOCO III: é obtido por multiplicar as combinações de tratamentos do bloco I por $\underline{b}$

BLOCO IV: é obtido por multiplicar as combinaçós de tratamentos do bloco I por

Sabemos pela regra (iii) que o produto das in terações confundidas tambēm estā confundido, i.é., ABC x ACD $=A^{2} B C^{2} D=B D$.

Sendo que os contriastes são:

$A B C=a+b+c+a b c+a d+b d+c d+a b c d-(1)-a b-a c-b c-d-a b d-a c d-b c d$

ou

$A B C=B L O C O I I+B L O C O I I I-B L O C O I-B L O C O I V$,

$A C D=a+a b+c+b c+d+b d+a c d+a b c d-(1)-b-a c-a b c-a d-a b d-c d-b c d$

ou

$A C D=B L O C O I I+B L O C O I V-B L O C O I-B L O C O I I I$, 
e,

$B D=(1)+a+c+a c+b d+a b d+b c d+a b c d+b-a b-a b c-d-a d-c d-a c d$

ou

$B D=B L O C O I+B L O C O I I-B L O C O I I I-B L O C O I V$.

Utilizando a geometria finita, e representando os fatores $A, B, C$ e $D$ respectivamente por $X_{1}, x_{2}, X_{3}$ e $x_{4}$, também podemos estruturar os quatro blocos acima.

Sabemos que a interação $A B C$ é a diferença entre as mëdias dos pontos representados por:

$$
\text { e } \begin{aligned}
& x_{1}+x_{2}+x_{3}=0(\bmod \cdot 2) \\
& x_{1}+x_{2}+x_{3}=1(\bmod \cdot 2)
\end{aligned}
$$

a interação ACD é a diferença entre as médias dos pontos representados por:

e

$$
\begin{aligned}
& x_{1}+x_{3}+x_{4}=0(\bmod \cdot 2) \\
& x_{1}+x_{3}+x_{4}=1(\bmod \cdot 2)
\end{aligned}
$$

e a interação generalizada $B D$ è a diferença entre as médias dos pontos representados por:

e

$$
\begin{aligned}
& x_{2}+x_{4}=0(\bmod 2) \\
& x_{2}+x_{4}=1(\bmod 2)
\end{aligned}
$$

Aplicando esta tëcnica, a estruturação do blo co sera: 
BLOCO I

$\mathrm{X}_{1}+\mathrm{X}_{2}+\mathrm{X}_{3}=0(\bmod .2)$
$\mathrm{x}_{1}+\mathrm{X}_{3}+\mathrm{X}_{4}=0(\bmod .2)$

(1) $=0000$

$\mathrm{ac}=1010$

$\mathrm{abd}=1101$

bcd $=0111$
BLOCO II

$x_{1}+x_{2}+x_{3}=0(\bmod .2)$

$x_{1}+x_{3}+x_{4}=1(\bmod .2)$

$\mathrm{d}=0001$

acd $=1011$

$a b=1100$

$\underline{b c}=0110$
BLOCO III

$\mathrm{x}_{1}+\mathrm{X}_{2}+\mathrm{X}_{3}=1 \quad(\bmod .2)$

$\mathrm{x}_{1}+\mathrm{x}_{3}+\mathrm{x}_{4}=0(\bmod .2)$

$\mathrm{b}=0100$

$a b c=1110$

$\mathrm{ad}=1001$

$\mathrm{cd}=0011$
BLOCO IV

$\mathrm{x}_{1}+\mathrm{x}_{2}+\mathrm{x}_{3}=1(\bmod .2)$

$\mathrm{x}_{1}+\mathrm{X}_{3}+\mathrm{x}_{4}=1(\bmod .2)$

bd $=0101$

$\operatorname{abcd}=1111$

$a=1000$

$c=0010$

Podemos observar que o 29 bloco é igual ao 19 , somando-se 1 a todos os elementos da coluna correspondente a D, o 39 bloco é igual ao 19 , somando-se 1 a coluna $B$, 0 4? bloco é igual ao 19, somando-se 1 a ambas colunas B e D.

o produto dos efeitos $A B C$ e $A C D$ é: $A B C x$ ACD $=A^{2} B C^{2} D=B D$.

Como jä vimos esse produto BD ë chamado de in teração generalizada, então podemos escreve-1a da seguinte maneira:

$$
\begin{aligned}
& \left(x_{1} x_{2} x_{3}\right)\left(x_{1} x_{3} x_{4}\right)=x_{1}^{2} x_{2} \cdot x_{3}^{2} x_{4}=x_{2} x_{4}, \text { desde que } \\
& x_{1}^{2}=x_{3}^{2}=1
\end{aligned}
$$

em cada unidade experimental.

A estrutura da anālise de variāncia para o ex perimento $2^{4}$ em 4 blocos com 4 tratamentos, repetido $r$ vezes, com o confundimento das interações $A B C, A C D$ e $B D$, é: 


\begin{tabular}{|c|c|}
\hline C. variação & G.L. \\
\hline$(B 10 \cos )$ & $(4 r-1)$ \\
\hline Repetições & $r-1$ \\
\hline$A B C, A C D, e B D$ & 3 \\
\hline Repetições $x$ interações & $3(r-1)$ \\
\hline (Tratamentos) & $(12)$ \\
\hline A & 1 \\
\hline B & 1 \\
\hline C & 1 \\
\hline D & 1 \\
\hline$A B$ & 1 \\
\hline $\mathrm{AC}$ & 1 \\
\hline $\mathrm{AD}$ & 1 \\
\hline BC & 1 \\
\hline $\mathrm{CD}$ & 1 \\
\hline $\mathrm{ABD}$ & 1 \\
\hline $\mathrm{BCD}$ & 1 \\
\hline$A B C D$ & 1 \\
\hline Residuo & $12(r-1)$ \\
\hline Total & $16 r-1$ \\
\hline
\end{tabular}

Para calcular as somas de quadrados, iremos utilizar o procedimento sistemático de YATES. 
De uma maneira geral, podemos apresentar :o confundimento nos experimentos fatoriais da série $2^{\text {n }}$ da seguinte maneira:

Nos experimentos fatoriais da série $2^{n}$, o con junto de todos efeitos, mais o elemento: unidade I é dado por:

$$
\mathrm{I}, \mathrm{A}, \mathrm{B}, \mathrm{C}, \ldots, \mathrm{AB}, \mathrm{AC}, \ldots, \mathrm{ABC}, \ldots
$$

Juntando a esses elementos a condição de que $\mathrm{A}^{2}=\mathrm{B}^{2}=\mathrm{C}^{2} \ldots=\mathrm{I}$, temos um grupo multiplicativo Abeliano de ordem $2^{n}$.

Um outro grupo anālogo é formado pelas combinaçoes de tratamentos com a condição que:

$$
a^{2}=b^{2}=c^{2}=\ldots=(1)
$$

A divisão de um experimento fatorial da série $2^{n}$ em blocos, pode ser efetuada da seguinte maneira:

$2^{1}$ blocos $\operatorname{com} 2^{\mathrm{n}-1}$ parcelas

$2^{2}$ blocos $\operatorname{com} 2^{\mathrm{n}-2}$ parcelas

$2^{3}$ blocos $\operatorname{com} 2^{n-3}$ parcelas

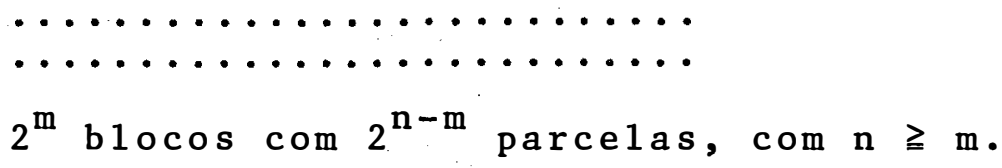

Suponha que estamos querendo dividir um expe- 
rimento $2^{\mathrm{n}}$ em 4 blocos, confundindo as interações $A B C$ e $A B D$. Assim, temos:

No 19 bloco são colocados todos os pontos com

$$
x_{1} x_{2} x_{3}=-1 \text { e } x_{1} x_{2} x_{4}=-1
$$

ou

$$
x_{1}+x_{2}+x_{3}=0(\bmod , 2) \text { e } x_{1}+x_{2}+x_{4}=0(\bmod \cdot 2)
$$

No 2 o bloco são colocados todos os pontos

$\operatorname{com}$

$$
x_{1} x_{2} x_{3}=-1 \quad \text { e } \quad x_{1} x_{2} x_{4}=+1
$$

ou

$$
x_{1}+x_{2}+x_{3}=0(\bmod .2) \text { e } x_{1}+x_{2}+x_{4}=1(\bmod .2)
$$

No 3 Q bloco são colocados todos os pontos com

$$
x_{1} x_{2} x_{3}=+1 \quad \text { e } \quad x_{1} x_{2} x_{4}=-1
$$

ou

$$
x_{1}+x_{2}+x_{3}=1(\bmod 2) \text { e } x_{1}+x_{2}+x_{4}=0(\bmod .2)
$$

No 4? bloco são colocados todos os pontos com

$$
x_{1} x_{2} x_{3}=+1 \quad \text { e } \quad x_{1} x_{2} x_{4}=+1
$$

ou

$$
\begin{aligned}
& x_{1}+x_{2}+x_{3}=1(\bmod \cdot 2) \text { e } x_{1}+x_{2}+x_{4}=1(\bmod .2) \\
& \text { Sendo que } x_{1} x_{2} x_{3}=x_{1} x_{2} x_{4}= \pm 1 \text {, então temos: } \\
& \left(x_{1} x_{2} x_{3}\right)\left(x_{1} x_{2} x_{4}\right)=x_{1}^{2} x_{2}^{2} x_{3} x_{4}=x_{3} x_{4}= \pm 1
\end{aligned}
$$


Portanto temos:

\begin{tabular}{lccccc}
\hline Efeitos & B1oco & B1oco & II & B1oco III & B1oco IV \\
\hline $\mathrm{X}_{1} \mathrm{X}_{2} \mathrm{X}_{3}$ & -1 & -1 & +1 & +1 \\
$\mathrm{X}_{1} \mathrm{X}_{2} \mathrm{X}_{4}$ & -1 & +1 & -1 & +1 \\
$\mathrm{x}_{3} \mathrm{X}_{4}$ & +1 & -1 & -1 & +1 \\
\hline
\end{tabular}

onde. cada bloco contém. $2^{n-2}$ pontos.

Observando a tabela acima, vemos que os contrastes dos totais de blocos são:

$$
\begin{aligned}
& B L . I I I+B L . I V-B L . I-B L . I I d a ̈ \text { o contraste } A B C \\
& B L . I I+B L . I V-B L . I-B L . I I I d a ̈ \text { o contraste ABD } \\
& B L . I+B L . I V-B L . I I-B L . I I I d a ̈ \text { ocontraste CD }
\end{aligned}
$$

Portanto, com este plano vemos que não pode mos estimar essas 3 interações, mas os outros efeitos serão estimados, pois nenhum outro efeito está confundido com efe to de blocos.

As interações $A B C$ e $A B D$ são chamadas de geradoras deste experimento. Em geral se existem $2^{\mathrm{m}}$ blocos no arranjo de confundimento, são necessärias m geradoras.

Generalizando, sejam $X$ e $Y$ dois efeitos que vão ser confundidos, então podemos dividir o experimento fatorial $2^{\text {n }}$ em $2^{2}$ blocos, cada um de tamanho $2^{n-2}$. Associamos 
ao bloco I todos os pontos que tem sinais negativos nos contrastes $\mathrm{X}$ e $\mathrm{Y}$, ao bloco II todos os pontos que tem sinais ne gativos no contraste $X$ e sinal positivo no contraste $Y$, e assim por diante. A interação generalizada XY também esta rá confundida com blocos .

Para dividirmos o experimento $2^{\mathrm{n}}$ em $2^{3}=8$ blo $\cos$ de tamanho $2^{n-3}$, tomamos um 39 efeito $W$, diferente de $X Y$. o bloco principal será constituido dos $2^{n-3}$ pontos que tenham sinais negativos nos contrastes $X, Y$ e W. Os sete efe tos confundidos serão: X, Y, XY, W, XW, YW, e XYW.

\subsubsection{CONFUNDIMENTO NA: SÉRIE $3^{n}$}

Para este ítem nös vamos utilizar a notação da interaça generalizada para os experimentos fatoriais da sèrie $2^{\text {n }}$, i.è., se os efeitos X e Y são confundidos, : eñtão XY também estará confundido, o qual será obtido pela multi plicação de X e Y, eliminando as letras em comum, i.é., igua lando a unidade, qualquer letra ao quadrado. Como foi visto está notação possibilita uma fácil enumeração do sistema de confundimento da série $2^{n}$.

Para o sistema $3^{n}$, nós vimos que os $3^{n}-1$ graus de 1 iberdade podem ser divididos em $\left(3^{n}-1\right) / 2$ efeitos, cada um com dois graus de 1 iberdade. A interação entre $A$ e $B$ é representada por $A B$ e $A B^{2}$. 
A regra da interação generalizada para o sistema $3^{\text {n }}$ é a seguinte: se os pares de graus de liberdade correspondentes a $X$ e $Y$ estão completamente confundidos, então os pares de graus de liberdade correspondentes a XY e $X Y^{2}$ também estão confundidos, onde a regra da multiplicação é a usual, com a condição que o cubo de qualquer letra é igual a unidade, ou seja,

$$
A^{3}=B^{3}=C^{3}=\cdots=I \text {. }
$$

Com esta notação, a ambiguidade que poderia aparecer se fosse tomada uma ordem diferente não existe, por que o efeito dado pela comparação $\lambda_{1} x_{1}+\lambda_{2} x_{2}+\lambda_{3} x_{3}+\ldots=0,1$, $2(\bmod .3)$ è a mesma comparação dada por $2\left(\lambda_{1} x_{1}+\lambda_{2} x_{2}+\lambda_{3} x_{3}+\ldots\right)$ $=0,2,1(\bmod .3)$, ou seja, a interação $A B C \ldots$ é a mesma in teração dada por $A^{2} B^{2} C^{2} \ldots$. Devemos 1 embrar que nós estamos adotando a regra que, em qualquer interação, o expoente da primeira letra será a unidade, i.é., se depois de uma mu 1 tiplicação, nós tivessemos a interação $A^{2} B C D$, nös a substi: tuimos pela seguinte interação: $\left(A^{2} B C D\right)^{2}=A^{4} B^{2} C^{2} D^{2}=A B^{2} C^{2} D^{2}$, pois nós estamos trabalhando com nümeros reduzidos a módulo 3. Desta maneira, nös obtemos uma enumeração completa e üni ca de todos efeitos.

KEMPTHORNE (1975), dá um exemplo de todas as maneiras de confundimento no fatorial $3^{3}$ em 9 blocos, cada um com 3 parcelas. A 1 ista dos 13 sistemas de confundimento cada um numa linha é dada na tabela a seguir. 


\begin{tabular}{|c|c|c|c|c|c|c|c|c|c|c|c|c|}
\hline A & B & $A B$ & $\mathrm{AB}^{2}$ & $\mathrm{C}$ & AC & $\mathrm{AC}^{2}$ & BC & $\mathrm{BC}^{2}$ & $A B C$ & $A B C^{2}$ & $\mathrm{AB}^{2} \mathrm{G}$ & $A B^{2} C^{2}$ \\
\hline $\mathrm{x}$ & $\mathrm{x}$ & $\mathrm{X}$ & $\mathrm{X}$ & & & & & & & & & \\
\hline $\mathrm{X}$ & & & & $\mathrm{x}$ & $x$ & $\mathrm{x}$ & & & & & & \\
\hline $\mathrm{X}$ & & & & & & & $\mathrm{X}$ & & $\mathrm{X}$ & & & $\mathrm{X}$ \\
\hline $\mathrm{x}$ & & & & & & & & $\mathrm{X}$ & & $\mathrm{x}$ & $\mathrm{x}$ & · \\
\hline & $\mathrm{x}$ & & & $\mathrm{x}$ & & & $\mathrm{X}$ & $\mathrm{x}$ & & & & \\
\hline & $\mathrm{x}$ & & & & $\mathrm{x}$ & & & & $\mathrm{X}$ & & $\mathrm{x}$ & \\
\hline & $\mathrm{x}$ & & & & & $\mathrm{x}$ & & & & $\mathrm{x}$ & & $\mathrm{x}$ \\
\hline & & $\mathrm{x}$ & & $\mathrm{X}$ & & & & & $\mathrm{X}$ & $\mathrm{X}$ & & \\
\hline & & $\mathrm{X}$ & & & $\mathrm{x}$ & & & $\mathrm{x}$ & & & & $\mathrm{x}$ \\
\hline & & $\mathrm{x}$ & & & & $\mathrm{X}$ & $\mathrm{X}$ & & & & $\mathrm{x}$ & \\
\hline & & & $\mathrm{X}$ & $\mathrm{x}$ & & & & & & & $\mathrm{X}$ & $\mathrm{x}$ \\
\hline & & & $\mathrm{X}$ & & $\mathrm{x}$ & & $\mathrm{X}$ & & & $\mathrm{x}$ & & \\
\hline & & & $\mathrm{x}$ & & & $\mathrm{x}$ & & $x$ & $\mathrm{x}$ & & & \\
\hline
\end{tabular}

Por exemplo, se tomarmos o primeiro sistema, nös vamos confundir A eB, e suas interações : generalizadas, que são $A B$ e $A B^{2}$.

Se tomarmos o ültimo sistema, nös temos:

$$
\mathrm{AB}^{2} \text { e } \mathrm{AC}^{2} \text {, dando as seguintes interações gene }
$$

ralizadas,

$$
\begin{aligned}
& A B^{2} \times A C^{2}=A^{2} B^{2} C^{2}=\left(A^{2} B^{2} C^{2}\right)^{2}=A^{4} B^{4} C^{4}=A B C, \\
& A B^{2} \times\left(A C^{2}\right)^{2}=A B^{2} \times A^{2} C^{4}=A^{3} B^{2} C=B^{2} C=\left(B^{2} C\right)^{2}=B C^{2} .
\end{aligned}
$$


EXEMPLO 1 .

Vamos tomar o experimento fatorial $3^{2}$ com uma repetição, confundindo $A B$. Assim, temos 3 blocos cada um com 3 combinações de tratamentos. As 9 combinações de trata mentos são: (1), $a, a^{2}, b, a b, a^{2} b^{2}, b^{2}, a b^{2}, a^{2} b^{2}, o u(00)$, $(10),(20),(01),(11),(21),(02),(12) ;(22)$.

Sabemos que os dois graus de liberdade corres pondente a $A B$, pode ser representados pelos contrastes entre os três grupos de combinações de tratamentos seguinte:

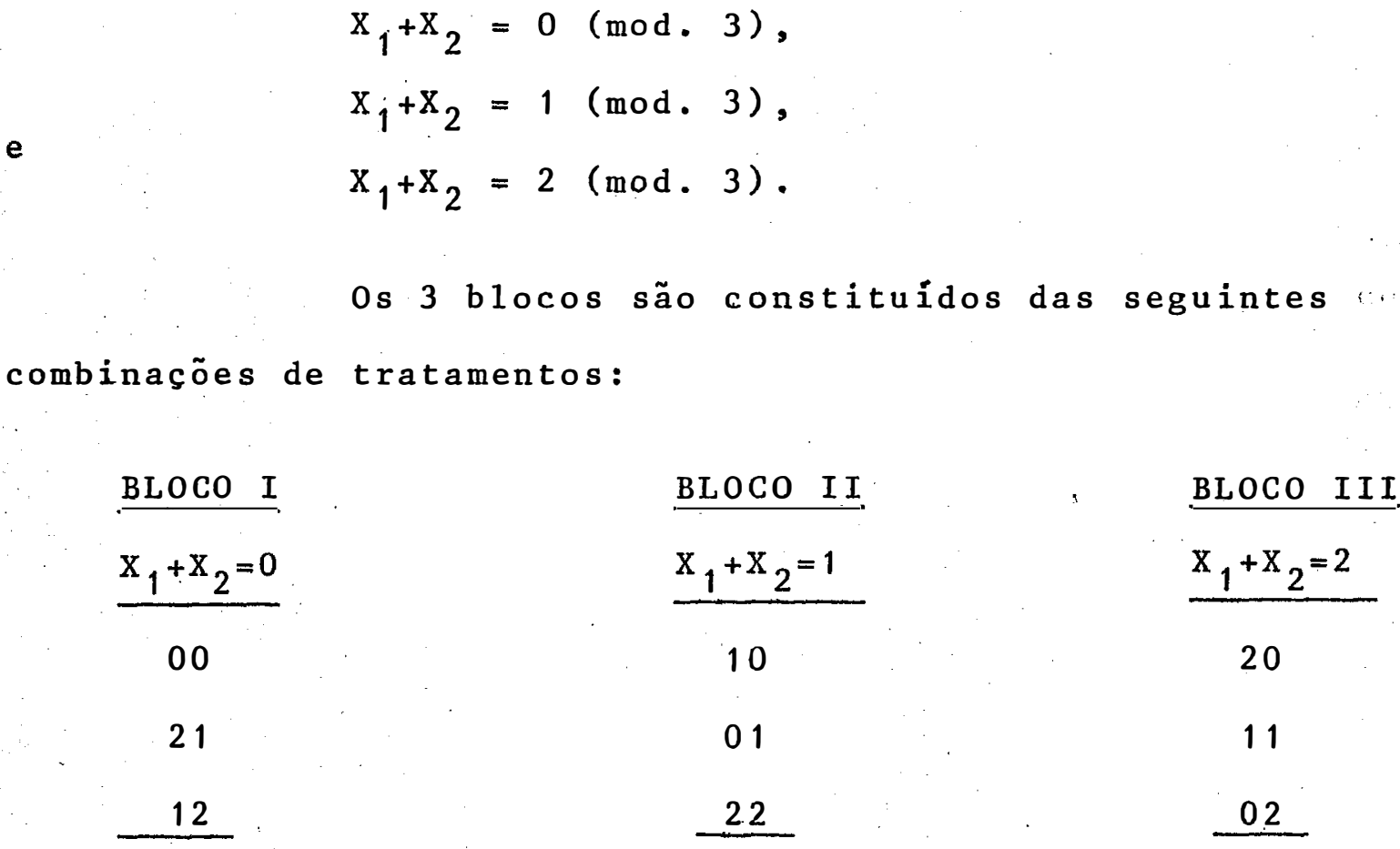

Se este experimento fosse repetido $r$ vezes, a estrutura da anălise de variāncia, com 2 graus de liberdade da interação $A B$ confundida é: 


\begin{tabular}{lc}
\hline C. variação & G.L. \\
\hline Blocos & $3 r-1$ \\
Repetičos & $r-1$ \\
AB & 2 \\
Repetičões x AB & $2(r-1)$ \\
Tratamentos & 6 \\
A & 2 \\
B & 2 \\
AB (não conf.) & $6(r-1)$ \\
Erro & $9 r-1$ \\
\hline Total & 2 \\
\hline
\end{tabular}

\section{EXEMPLO 2.}

Vamos tomar o experimento fatorial $3^{3} \mathrm{com}$ uma repetição, e vamos confundir $\mathrm{AB}$ e $\mathrm{AC}^{2}$. Assim; temos 9 blocos, cada um com 3 combinações de tratamentos. As 27 combinações de tratamentos são:

$$
\begin{aligned}
& \text { (1) }=000 \\
& c=001 \\
& c^{2}=002 \\
& a=100 \\
& a c=101 \\
& a c^{2}=102 \\
& a^{2}=200 \\
& a^{2} c=201 \\
& a^{2} c^{2}=202 \\
& \mathrm{~b}=010 \\
& \mathrm{bc}=011 \\
& \mathrm{bc}^{2}=012 \\
& a b=110 \\
& a b c=111 \\
& a b c^{2}=112 \\
& a^{2} b=.210 \\
& a^{2} b c=211 \\
& a^{2} b c^{2}=212
\end{aligned}
$$




$$
\begin{aligned}
& b^{2}=020 \\
& b^{2} c=021 \\
& b^{2} c^{2}=022 \\
& a b^{2}=120 \\
& a b^{2} c=121 \\
& a b^{2} c^{2}=122 \\
& a^{2} b^{2}=220 \\
& a^{2} b^{2} c=221 \\
& a^{2} b^{2} c^{2}=222
\end{aligned}
$$

Como estamos confundindo $\mathrm{AB}$ e $\mathrm{AC}^{2}$, então tambẻm estarão confundidas as seguintes interações generaliza -

\begin{tabular}{|c|c|c|c|c|c|c|c|c|}
\hline BLOCO I & BLOCO II & BLOCO III & BLOCO IV & BLOCO V & BLOCO VI & BLOCO VII & BLOCO VII & BLOCO IX \\
\hline$x_{1}+x_{2}=0$ & $x_{1}+x_{2}=0$ & $x_{1}+x_{2}=0$ & $x_{1}+x_{2}=1$ & $x_{1}+x_{2}=1$ & $x_{1}+x_{2}=1$ & $x_{1}+x_{2}=2$ & $x_{1}+x_{2}=2$ & $x_{1}+x_{2}=0$ \\
\hline$x_{1}+2 x_{3}=0$ & $x_{1}+2 x_{3}=1$ & $x_{1}+2 x_{3}=2$ & $x_{1}+2 x_{3}=0$ & $x_{1}+2 x_{3}=1$ & $x_{1}+2 x_{3}=2$ & $x_{1}+2 x_{3}=0$ & $x_{1}+2 x_{3}=1$ & $x_{1}+2 x_{3}=2$ \\
\hline 000 & 002 & 001 & 010 & 012 & 011 & 020 & 022 & 021 \\
\hline 121 & 120 & 122 & 101 & 100 & 102 & 111 & 110 & 112 \\
\hline 212 & 211 & 210 & 222 & 221 & 220 & 202 & 201 & 200 \\
\hline
\end{tabular}
das :

$$
\begin{aligned}
& \mathrm{AB} \times \mathrm{AC}^{2}=\mathrm{A}^{2} \mathrm{BC} \mathrm{C}^{2}=\left(\mathrm{A}^{2} \mathrm{BC}^{2}\right)^{2}=\mathrm{A}^{4} \mathrm{~B}^{2} \mathrm{C}^{4}=A B^{2} \mathrm{C}, \mathrm{e} \\
& \mathrm{AB} \times\left(\mathrm{AC}^{2}\right)^{2}=\mathrm{AB} \times \mathrm{A}^{2} \mathrm{C}^{4}=\mathrm{A}^{3} \mathrm{BC}=\mathrm{BC}
\end{aligned}
$$

Os 9 blocos são dados pelas soluções das equa cões $x_{1}+x_{2}=i(\bmod \cdot 3)$ e $x_{1}+2 x_{3}=j(\bmod \cdot 3)$, onde $i$ e $j$ a sumem os valores 0,1 e 2. 0 bloco principal ou o subgrupo intra-bloco é aquele constituido pelas combinações de tratamentos que satisfazem as equações, $X_{1}+X_{2}=0(\bmod \cdot 3)$ e $x_{1}+2 x_{3}=0(\bmod \cdot 3)$. Assim temos: 
Podemos observar que:

BLOCO II: E obtido somando-se 2 a todos os elementos da 3 a coluna do b1oco I.

BLOCO III: E obtido somando-se $i$ a todos os elementos da 3a coluna do b1oco I.

BLOCO IV: E obtido somando-se 1 a todos os elementos da 2a coluna do b1oco I.

BLOCo V: E obtido somando-se 1 e 2 às colunas B e C, respectivamente do b1oco I.

BLOCO VI: E obtido somando-se 1 e 1 as colunas B e C, respec tivamente do b1oco I.

BLOCO VII: E obtido somando-se 2 a todos os lelementos da 2a coluna do b1oco I.

BLOCO VIII: E obtido somando-se 2 e 2 as colunas B e C respectivamente do b1oco I.

BLOCO IX: E obtido somando-se 2 e 1 as colunas $B$ e C, respec tivamente ao b1oco I.

A estrutura da anāi ise de variāncia para este experimento fatorial $3^{3}$, no qual estão confundidos os 2 graus liberdade correspondente às seguintes interaçóes $A B, \quad A C^{2}$, $\mathrm{AB}^{2} \mathrm{C}$ e $\mathrm{BC}$, sendo repetido $\underline{\mathrm{r}}$ vezes, é: 


\begin{tabular}{|c|c|}
\hline C. Variação & G.L. \\
\hline B1ocos & $9 r-1$ \\
\hline Repetições & $\mathrm{r}-1$ \\
\hline$A B, A C^{2}, A B^{2} C$ e $B C$ & 8 \\
\hline Repetições $x$ Interações & $8(r-1)$ \\
\hline Tratamentos & 18 \\
\hline A & 2 \\
\hline B & 2 \\
\hline c & 2 \\
\hline $\mathrm{AB}^{2}$ & 2 \\
\hline $\mathrm{AC}$ & 2 \\
\hline $\mathrm{BC}^{2}$ & 2 \\
\hline $\mathrm{ABC}$ & 2 \\
\hline $\mathrm{ABC}^{2}$ & 2 \\
\hline $\mathrm{AB}^{2} \mathrm{C}^{2}$ & 2 \\
\hline Erro & $18(r-1)$ \\
\hline Tota 1 & $27 r-1$ \\
\hline
\end{tabular}

3.1.3. EXPERIMENTOS FATORIAIS COM UMA ÚNICA REPETIС̆̃O

Em muitas pesquisas exploratörias, empregando experimentos fatoriais, o nümero de combinacões de tratamentos è às vezes muito grande, e como sabemos, uma desvantagem 
dos experimentos fatoriais è que o nümero de combinaçós de tratamentos crescem rapidamente a medida que aumenta o nümero de fatores.

Este fato limita o nümero de repetições a ser empregado, devido a dificuldade operacional de ensaios muito grande, bem como a escasses de recursos, que na maioria das vezes são suficientes somente para uma única repetição.

Se somente uma ünica repetição é usada, o experimento não será capaz de fornecer uma estimativa do erro, pelo procedimento comum de comparar repetiçós. Mas, em experimentos grandes existirä muitas interações entre 3 ou mais fatores (interações de ordem elevada), que podem em muitos casos serem considerados como sendo não significativos. Por tanto, se certas interações de ordem elevada são insignifi cantes, seu quadrado médio na anälise de variāncia se comporta rá exatamente como componentes do quadrado médio do erro, e portanto, podem ser usadas para fornecer uma estimativa do erro.

Existem criticas na inclusão das interações de ordem elevada na estimativa do erro, pois se algumas destas interações não forem insignificantes, i.è., tenham alguma grandeza apreciável, o quadrado médio do erro que : serä usado superestimarā a verdadeira variāncia do erro. Devemos notar que até mesmo, sendo algumas destas interações de or- 
dem elevada de grandeza apreciável, o pesquisador ainda está em uma posição muito melhor do que se tivesse omitido inteiramente o fator, pois qualquer uma destas interações (exceto aquelas que estão confundidas), podem mais tarde ser isoladas e examinadas.

Este esquema de usar somente uma ünica repetí ção è particularmente ütil em experimentos no campo agríco1a. Vejamos no caso do fatoria $12^{6}$ como ficaria o esquema da anảise de variância se desejassemos confundir as interacões $A B C D, C D E F$ e $A B E F$ com 4 blocos, cada um com 16 combinações de tratamentos.

C. variação

G.L .

B1ocos

Efeitos principais

Interações de 2 fatores 15

Interações de 3 fatores 20

Erro (interações de ordem elevadas) $\quad 19$

Tota 1

63

Em pesquisa cientifica com maior precisão, uma única repetição só pode ser considerada adequada se o nümero de fatores for igual ou maior do que 6 , segundo KEMPTHORNE (1975). 


\subsubsection{EFICIENCIA}

A técnica da eficiência é essencialmente a mesma para todos tipos de delineamentos.

Para os experimentos com confundimento temos que: sejam $\sigma_{b}^{2}$ a variância do erro experimental para o experi mento com confundimento e $\sigma_{r}^{2}$ a variancia do erroexperimen:tal para o experimento em blocos completos ao acaso. Assim, a eficiência serä:

$$
E=\frac{\sigma_{r}^{2}}{\sigma_{b}^{2}}
$$

Nós conhecemos que o quadrado médio do erro para o experimento com confundimento é um estimador não viciado de $\sigma_{b}^{2}$. A questão ägora é estimar $\sigma_{r}^{2}$ para o experimento em blocos completos ao acaso. Dos resultados da análise de variância de um experimento com confundimento, a estimatí va de $\sigma_{r}^{2}$ é feita da seguinte maneira:

\subsubsection{NO CASO DE UMA ÚNICA REPETIÇÃO}

$$
\hat{\sigma}_{r}^{2}=\frac{n_{b} Q M_{B 1 \text { ocos }}+n_{e} Q M_{E r r o}}{n_{b}+n_{e}} \quad, \quad \text { onde }
$$

$\mathrm{n}_{\mathrm{b}}$ é o nümero de graus de libérdade para blocos;

$\mathrm{n}_{e}$ é o número de graus de 1 iberdade total menos $\mathrm{n}_{\mathrm{b}}$. 


$$
\begin{aligned}
& \text { Portanto, estimamos E como: } \\
& E=\frac{\partial_{r}^{2}}{\partial_{b}^{2}}=\frac{\hat{\sigma}_{r}^{2}}{Q M_{E r r o}} \Rightarrow E=\frac{n_{b} Q_{B 1 o c o s}+n_{e} Q_{E r r o}}{\left(n_{b}+n_{e}\right) M_{E r r o}}
\end{aligned}
$$

3.1.4.2. NO CASO DE MAIS DE UMA REPETIÇÃO

Se o confundimento é completo, a formula acima è mantida, com pequenas modificações, isto é:

$$
\partial_{r}^{2}=\frac{n_{b} Q M_{B 1 o c o s} d \cdot \text { repetição }}{+n_{e} Q M_{\text {Erro }}},
$$

onde

$n_{b}$ è o número de graus de liberdade para blocos dentro de re petições

$n_{e}$ é o nümero total de graus de liberdade, menos o número de graus de 1 iberdade para repetições e menos $n_{b}$.

A estimativa $\hat{\sigma}_{r}^{2}$ estä sujeita a suposição que as interações que são confundidas comblocos são insignifi cantes. Desde que o confundimento seja completo, nenhum método de estimação evita esta suposição. Se estas interações não são insignificantes, o cälculo superestima o ganho em precisão do confundimento.

JOHN NETER e WILLIAM WASSERNAN (1974), apresenta uma modificação que frequentemente é usada, é aplica 
da para avaliar qualquer experimento em relação a outro, isto é :

$$
\hat{E}^{\prime}=\frac{\left(d f_{2}+1\right)\left(d f_{1}+3\right)}{\left(d f_{2}+3\right)\left(d f_{1}+1\right)} \bar{E} \quad, \quad \text { onde }
$$

df 1 representa os graus de liberdade para o erro experimenta1 do delineamento base;

$\mathrm{df}_{2}$ representa os graus de liberdade para o erro experimental cuja eficiência esta sendo avaliada.

Segundo os dois autores acima citados, esta modificação tem pouco efeito, a não ser que os graus de 1 iberdade para o erro experimental em ambos experimentos sejam muito pequeno.

\subsubsection{FATORIAL FRACIONADO}

Como temos visto, até agora diṣcutimos experi mentos fatoriais completos, e sabemos que esses experimentos são muito apropriados para pesquisas exploratórias, e que em tais pesquisas o nümero de fatores a serem testados é razoavelmente grande. As vezes até uma ünica repetição de um experimento fatorial estä além dos recursos do pesquisador. As sim em situações como esta, um experimento que consiste somente de uma parte da repetição completa é de considerável valor. 
A técnica usada para selecionar uma fração das combinações de tratamentos de um experimento fatorial foi introduzido por FINNEY (1945). Essa técnica é conhecida como fatorial fracionado e faz parte da teoria de confundimento.

Os fatoriais fracionados são empregados nos ca sos em que alguns efeitos podem ser considerados como insignificantes, e serem confundido como outros efeitos de inte resse no experimento, reduzindo o nümero de combinaçós de tratamentos.

Este esquema è limitado em sua it utiližação, pois todos efeitos estão associados a outro efeito. Por isso um planejamento cuidadoso deve ser feito para garantir o valor do experimento. Apesar disso, o fatorial fracionado foi usado com vantagens em experimentos em que alguns ou todos fatores estão em dois níveis somente.

Os fatoriais fracionados tem grande utilidade em pesquisas àgricolas, físicas, biológicas, químicas, indus triais, etc.

BOX e HUNTER (1961), classificaram os experimentos fatoriais fracionados em. 3 tipos, que são:

a. Experimentos de resolução III: são aqueles constituidos de frações, nas quais alguns dos efeitos princi pais tem como alias (ou estão confundidos) interações de dois fatores, sendo portanto estimáveis apenas quando estas 
são ómitidas.

b. Experimentos de resolução IV: são aqueles constituidos de frações nas quais todos os efeitos principais. são estimāveis, independente das interações de dois fatores serem ou não omitidas, mas algumas dessas interações constituem pares de alias.

c. Experimentos de resolução v: são aqueles constituidos de frações nas quais todos os efeitos principais e interações de dois fatores são estimãveis.

\subsubsection{FATORIAL FRACIONADO NO SISTEMA $2^{\text {n }}$}

Vamos iniciar o estudo do fatorial fracionado no sistema $2^{n}$, com um exemplo simples, para ver o que aconte ce quando o experimento contém parte de uma rẹpetição. Será considerado o fatorial $2^{3}$, com os fatores A, B e C por sua simplicidade.

Sabemos que o fatorial $2^{3}$ tem oito combinações de tratamentos numa repetição completa, i.ë., (1), a, $b, a b, c, a c, b c e a b c$.

Vamos supor que são testados somente as 4 com binações de tratamentos $a, b, c$ e abc, ou seja, exatamente a 
metade do fatorial completo. Assim, os efeitos principais e as interações são estimadas de acordo com a tabela abaixo:

\begin{tabular}{cccccc}
\hline Efeitos Fatoriais & \multicolumn{4}{c}{ Combinacoes de tratamentos } \\
\cline { 2 - 5 } & a & b & c & abc \\
\hline A & + & - & + & - & + \\
B & - & - & + & + \\
AB & - & - & + & + \\
C & - & + & - & + \\
AC & + & - & - & + \\
BC & + & + & + & + \\
ABC & & +
\end{tabular}

Para o efeito principal de A, nós somamos as produções das combinações de tratamentos que contém a e subtraimos aquelas que não o contém, ou seja,

$$
A=a+a b c-b-c
$$

e, similarmente para B e C, ou seja:

$$
\begin{aligned}
& B=b+a b c-a-c, \\
& c=c+a b c-a-b .
\end{aligned}
$$

Pela tabela acima vemos que as comparações $A$, B e C são mutuamente ortogonais, assim as estimativas dos 3. 
efeitos principais são independentes.

$$
\text { Para calcularmos a interação } A B \text { somamos }
$$

produções das combinações de tratamentos que contēm um nümero par das letras a e $\underline{b}$, e subtraimos aquelas com um número impar, ou seja,

$$
A B=a b c+c-a-b \text {, }
$$

similarmente para AC e BC, ou seja,

$$
\begin{aligned}
& A C=a b c+b-a-c, \quad e \\
& B C=a b c+a-b-c .
\end{aligned}
$$

Observamos que a mesma quantidade que estima A, tambēm estima BC, então dizemos que A e BC são alias. Anạ logamente isto também acontece com B e AC, e C e AB. Então podemos escrever que $A=B C, B=A C$ e $A C=A B$. Este conjunto é o que FINNEY (1945) chama de conjunto de aliases.

A interação $A B C$ não pode ser eśtimada. Se to das as oito combinações de tratamentos estivessem disponiveis, então ABC seria dada por:

$$
A B C=a b c+a+b+c-a b-a c-b c-(1)
$$

Assim, vemos que escolhemos as 4 combinações

de tratamentos que levam o sinal + nesta expressão, para ser a metade da repetição. Neste caso, ABC é chamado de con traste de definição ou relação de identidade, pois foi o con 
traste usado para dividir o fatorial em duas partes, e serä denotado por:

$$
I=+A B C
$$

Resumindo, o uso da fração $2^{-1}$ acarreta na perda total da interação $A B C$, e confunde totalmente cada efeito principal com uma interaça de 2 fatores.

Sempre que utilizamos experimento fatoriàl fra cionado esta confusão está presente. No nosso caso, temos um efeito aparente de $(A=B C)$, i.é., não temos como descobrir se esse efeito é devido a A, BC, ou a ambos. 0 motivo da confusão é devido ao fato de que todo efeito fatorial sempre tem uma ou mais aliases.

Devemos observar que o problema de decidir pa ra qual alias o efeito é atribuido, é dificuldade que o pesquisador sempre enfrentará na hora de interpretar os resulta dos.

Em alguns tipos de pesquisa, a experiência an terior com os mesmos fatores, ou um conhecimento da natureza de seus efeitos, pode levar o pesquisador a decidir quais as interações que podem ser consideradas como insignificantes. Então a confusão desaparecerä, e nös concluimos que um efeito aparente de $A$ é de fato devido a A, e igualmente para B e C. Com estas suposições, a metade da repetição fornece estí mativas independentes dos 3 efeitos principais. 
No caso de concluirmos o experimento num tempo relativamente pequeno, podemos realizar um novo experimen to utilizando as outras combinaços de tratamentos omitidas no primeiro.

Juntando os resultados dos dois experimentos obtemos uma repetição completa, que dá estimativas independentes de todos efeitos. E claro que este esquema é menos praticavel em experimentos que tomam um tempo longo para pro duzir seus resultados.

A fim de reduzir os riscos de conclusões erra das, devemos conhecer os efeitos fatoriais nos quais estamos interessados. Para isso apresentamos a seguinte regra:

REgRA 1. (COCHRAN e $\operatorname{cox}, 1957)$ : "No sistema $2^{n}$, a alias de qualquer efeito fatorial é a interação generalizada desse efeito com a relação de identidade".

No exemplo acima, temos que a alias de A serä:

$$
A \times I=A \times A B C \Longrightarrow A=A^{2} B C \Longrightarrow A=B C
$$

Agora, se nós tivessemos instalado o experi mento com as combinações de tratamentos (1), ab, ac e bc, referente a outra parte, teriamos a seguinte tabela: 


\begin{tabular}{|c|c|c|c|c|c|}
\hline \multirow{2}{*}{ Efeitos } & \multirow{2}{*}{ Fatoriais } & \multicolumn{4}{|c|}{ Combinaçoes de tratamentos } \\
\hline & & (1) & $a b$ & ac & $b c$ \\
\hline & $\mathrm{A}$ & - & + & + & - \\
\hline & B & - & + & - & + \\
\hline & $A B$ & ist & + & - & - \\
\hline & C & - & - & + & + \\
\hline & $\mathrm{AC}$ & + & - & + & - \\
\hline & BC & + & - & - & + \\
\hline & $\mathrm{ABC}$ & - & - & - & - \\
\hline
\end{tabular}

Observamos que o conjunto de aliases ë:

$$
A=-B C ; B=-A C \text { e } \quad C=-A B
$$

Isto pode ser comprovado pela tabela acima,

ou seja,

$$
\left.\begin{array}{l}
A=a b+a c-(1)-b c, \quad e \\
B C=(1)+b c-a b-a c
\end{array}\right\} \Rightarrow A=-B C
$$

o único efeito que não pode ser estimado é a interação $A B C$, e será chamada de contraste de definição ou relação de identidade, e será denotado por: $I=-A B C$.

Em resumo, quando dividimos o fatorial $2^{3}$ em duas partes, tomando $A B C$ como o contraste de dedinição, temos: 


\begin{tabular}{cc}
\multicolumn{2}{c}{$I= \pm A B C$} \\
$\begin{array}{c}\text { Fração } \\
+A B C\end{array}$ & Fração 2 \\
a & -ABC \\
b & ab \\
$c$ & $a c$ \\
abc & bc \\
\hline
\end{tabular}

A fração 2 será o subgrupo intrabloco, e serã sempre a fração que contém o tratamento controle, denotado por (1), i.é., todas as combinações de tratamentos com o número par de letras em comum com o contrastede definição.

A partir do subgrupo intrabloco podemos deter minar as combinações de tratamentos da outra fração, i.é.,es colhemos qualquer combinação de tratamento que não está neste subgrupo e multiplicamos cada combinação de tratamento dés se subgrupo por esta, lembrando que qualquer letra ao quadra do é igual a 1.

Para determinar o sinal da relação de identidade, notamos que na fração contendo a combinação de tratamento em que aparecem todas as letras da relação de identidade leva o sinal + , e portanto a outra fraça leva o sinal

Observamos que os autores consideram que os fatoriais fracionados para ter valor prätico é preciso "que 
os efeitos principais e as interações de 2 fatores sejam es-. timados, isto quer dizer que os efeitos principais e as inte rações de 2 fatores tenham como aliases interações de 3 ou mais fatores.

Agora, vamos utilizar a notação de geometria finita para os fatoriais fracionados.

3.1.5.1.1. Descrição do bloco principal, fracão $2^{n-k}$ e conjunto de alias

Para descrever o bloco principal e o conjunto de alias nós vamos tomar alguns exemplos:

EXEMPLO 1. Dividindo-se o delineamento $2^{6}$ em 2 blocos com $2^{5}$ combinações de tratamentos, cada bloco constituirá uma fração, chamada de fração $2^{6-1}$.

Sejam A, B, C, D, E e F os seis fatores repre sentados por $x_{1}, x_{2}, x_{3}, x_{4}, x_{5}$ e $x_{6}$, respectivamente, e vamos confundir a interação $A B C D E F$.

o bloco principal ou o subgrupo intrabloco consiste de todas as combinações de tratamento satisfazendo a equação:

$$
x_{1}+x_{2}+x_{3}+x_{4}+x_{5}+x_{6}=0
$$


Então temos:

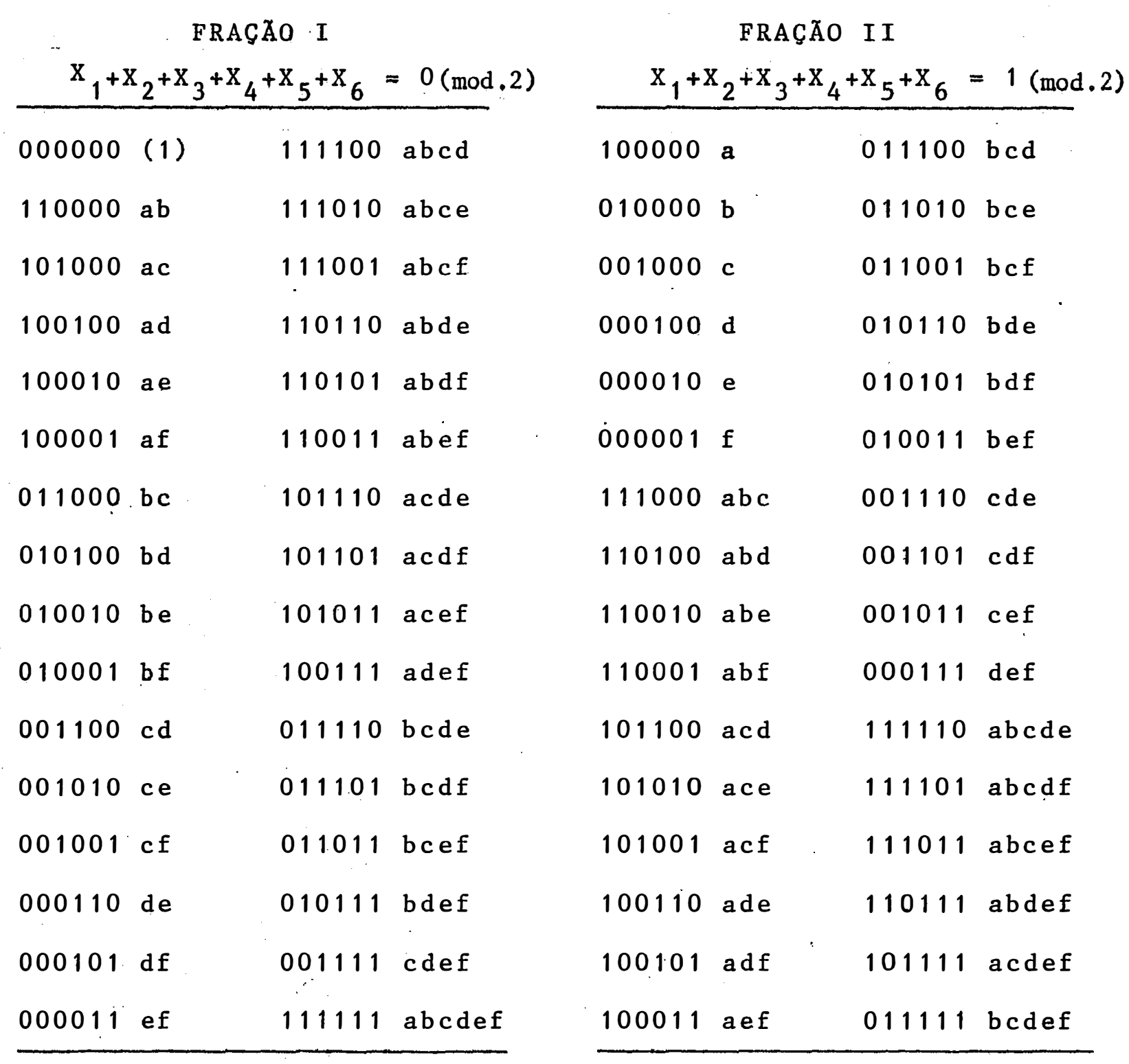

A fração. II é formada a partir da fração I, mu1tiplicando-se as combinações de tratamentos da fração I por qualquer combinação que não pertence a ela, por exemplo, a combinação a, ou de outro modo, adicionando-se 1 à coluna correspondente ao fator A. 
Tambëm poderiamos escrever o bloco principal da seguinte maneira: ele consiste dos pontos $x_{1} X_{2} X_{3} X_{4} X_{5} X_{6}=+1$. Multip1icando esta equação por $x_{6}$, e notando que $x_{6}^{2}=1$, temos que:

$$
x_{6}=+x_{1} x_{2} x_{3} x_{4} x_{5}
$$

Podemos tambëm escrever o b1oco principal com o delineamento bäsico $2^{5}$ nos fatores A, B, C, D e E, e depois adicionamos o outro fator $F$, colocando $X_{6}=X_{1}+X_{2}+X_{3}+X_{4}+X_{5}$ (mod. 2), ou $x_{6}=+x_{1} x_{2} x_{3} x_{4} x_{5}$. Analogamente, o segundo b1oco (fração II) serä obtido fazendo $X_{6}=\div X_{1} X_{2} X_{3} X_{4} X_{5}$, ou seja $x_{1}+x_{2}+x_{3}+x_{4}+x_{5}+x_{6}=1(\bmod \cdot 2)$ 
.74.

\begin{tabular}{|c|c|c|c|c|c|c|}
\hline A & & C & $D:$ & E & $F$ & \\
\hline$x_{1}$ & $\mathrm{x}_{2}$ & $\mathrm{x}_{3}$ & $x_{4}$ & $x_{5}$ & $x_{6}=x_{1}+x_{2}+x_{3}+x_{4}+x_{5}$ & $(\bmod 2):$ \\
\hline 0 & 0 & 0 & 0 & 0 & 0 & (1) \\
\hline 1 & 0 & 0 & 0 & 0 & 1 & af \\
\hline 0 & 1 & 0 & 0 & 0 & 1 & $b f$ \\
\hline 1 & 1 & 0 & 0 & 0 & 0 & $a b$ \\
\hline 0 . & 0 & 1 & 0 & 0 & 1 & $c f$ \\
\hline 1 & 0 & 1 & 0 & 0 & 0 & ac \\
\hline 0 & 1 & 1 & 0 & 0 & 0 & $\mathrm{bc}$ \\
\hline 1 & 1 & 1 & 0 & 0 & 1 & $a b c f$ \\
\hline 0 & 0 & 0 & 1 & 0 & 1 & $d f$ \\
\hline 1 & 0 & 0 & 1 & 0 & 0 & $\mathrm{ad}$ \\
\hline 0 & 1 & 0 & 1 & 0 & 0 & $b d$ \\
\hline 1 & 1 & 0 & 1 & 0 & 1 & $a b d f$ \\
\hline 0 & 0 & 1 & 1 & 0 & 0 & $c d$ \\
\hline 1 & 0 & 1 & 1 & 0 & 1 & $\operatorname{acd} f$ \\
\hline 0 & 1 & 1 & 1 & 0 & 1 & $b c d f$ \\
\hline 1 & 1 & 1 & 1 & 0 & 0 & $a b c d$ \\
\hline 0 & 0 & 0 & 0 & 1 & 1 & ef \\
\hline 1 & 0 & 0 & 0 & 1 & 0 & ae \\
\hline 0 & 1 & 0 & 0 & 1 & 0 & be \\
\hline 1 & 1 & 0 & 0 & 1 & 1 & abef \\
\hline 0 & 0 & 1 & 0 & 1 & 0 & $\mathrm{ce}$ \\
\hline 1 & 0 & $1 i$ & 0 & 1 & 1 & acef \\
\hline 0 & 1 & 1 & 0 & 1 & 1 & bcef \\
\hline 1 & 1 & 1 & 0 & 1 & 0 & $a b c e$ \\
\hline 0 & 0 & 0 & 1 & 1 & 0 & de \\
\hline 1 & 0 & 0 & 1 & 1 & 1 & adef \\
\hline 0 & 1 & 0 & 1 & 1 & 1 & bdef \\
\hline 1 & 1 & 0 & 1 & 1 & 0 & $a b d e$ \\
\hline 0 & 0 & 1 & 1 & 1 & 1 & cdef \\
\hline 1 & 0 & 1 & 1 & 1 & 0 & acde \\
\hline 0 & 1 & 1 & 1 & 1 & 0 & bcde \\
\hline 1 & 1 & 1 & 1 & 1 & 1 & abcdef \\
\hline
\end{tabular}


No delineamento $2^{6}$ descrito acima, o bloco principal è a fração definida por: $I=+A B C D E F$
A outra fração è $I=-A B C D E F$

O termo principal è usado, algumas vezes, para indicar a fração que contēm (1), por analogia ao bloco principal.

No exemplo acima, temos os seguintes conjuntos de aliases, tomando a fração $I=+A B C D E F$.

\begin{tabular}{|c|c|c|c|c|c|}
\hline $\begin{array}{l}\text { Efeitos } \\
\text { Principais }\end{array}$ & Alias & $\begin{array}{c}\text { Interações } \\
\text { de } 2 \\
\text { fatores }\end{array}$ & Alias & $\begin{array}{c}\text { Interações } \\
\text { de } 3 \\
\text { fatores }\end{array}$ & Alias \\
\hline A & B.CDEF & $A B$ & CDEF & $\mathrm{ABC}$ & $\mathrm{DEF}$ \\
\hline B & $\mathrm{ACDEF}$ & $\mathrm{AC}$ & BDEF & $\mathrm{ABD}$ & CEF \\
\hline C & $\mathrm{ABDEF}$ & $\mathrm{AD}$ & B CEF & $\mathrm{ABE}$ & $\mathrm{CDF}$ \\
\hline D & $A B C E F$ & $\mathrm{AE}$ & BCDF & $A B F$ & $\mathrm{CDE}$ \\
\hline E 1 & $A B C D F$ & $\mathrm{AF}$ & BCDE & $A C D$ & BEF \\
\hline \multirow[t]{10}{*}{ F } & $A B C D E$ & B C & ADEF & $\mathrm{ACE}$ & $\mathrm{BDF}$ \\
\hline & & BD & ACEF & $\mathrm{ACF}$ & $\mathrm{BDE}$ \\
\hline & & $\mathrm{BE}$ & $\mathrm{ACDF}$ & $\mathrm{ADE}$ & $\mathrm{BC} F$ \\
\hline & & B F & $A C D E$ & $\mathrm{ADF}$ & B CE \\
\hline & & $C D$ & $\mathrm{ABEF}$ & $\mathrm{AEF}$ & BCD \\
\hline & & $\mathrm{CE}$ & $\mathrm{ABDF}$ & & \\
\hline & & $C F$ & $A B D E$ & & \\
\hline & & $\mathrm{DE}$ & $A B C F$ & & \\
\hline & & $\mathrm{DF}$ & $A B C E$ & & \\
\hline & & E F & $A B C D$ & & \\
\hline
\end{tabular}

Pela tabela acima, vemos que os efeitos prin 
cipais tem como alias, interaçóes de 5 fatores e as interações de 2 fatores tem como alias interações de 4 fatores.

Se pudermos considerar as interações de 3 ou mais fatores como insignificantes, os efeitos principais e as interações de 2 fatores são estimãveis, e para a estimatí va do erro temos 10 graus de 1 iberdade, relativo as 20 interações de 3 fatores. Assim, o esquema do quadro da análise de variância será:
C. variação
G.L .

Efeitos principais

Interações de 2 fatores

Residuo

10

Tota 1

EXEMPLO 2. Dividindo-se o delineamento $2^{8}$ em $2^{2}$ blocos com $2^{6}$ unidades, cada bloco constituirä uma fração, chamada de fração $2^{8-2}$.

Sejam A, B, C, D, E, F, G e H, os oito fatores representados por $x_{1}, x_{2}, x_{3}, x_{4}, x_{5}, x_{6}, x_{7}$ e $x_{8}$ respec tivamente. Confundindo por exemplo ABCDG e ABEFH, também es tá confundido CDEFGH (3 g.1.). 
o bloco principal ou o subgrupo intrabloco consiste de todas as combinações de tratamentos satisfazerido

$$
x_{1}+x_{2}+x_{3}+x_{4}+x_{7}=0(\bmod \cdot 2)
$$

e

$$
x_{1}+x_{2}+x_{5}+x_{6}+x_{8}=0(\bmod \cdot 2)
$$

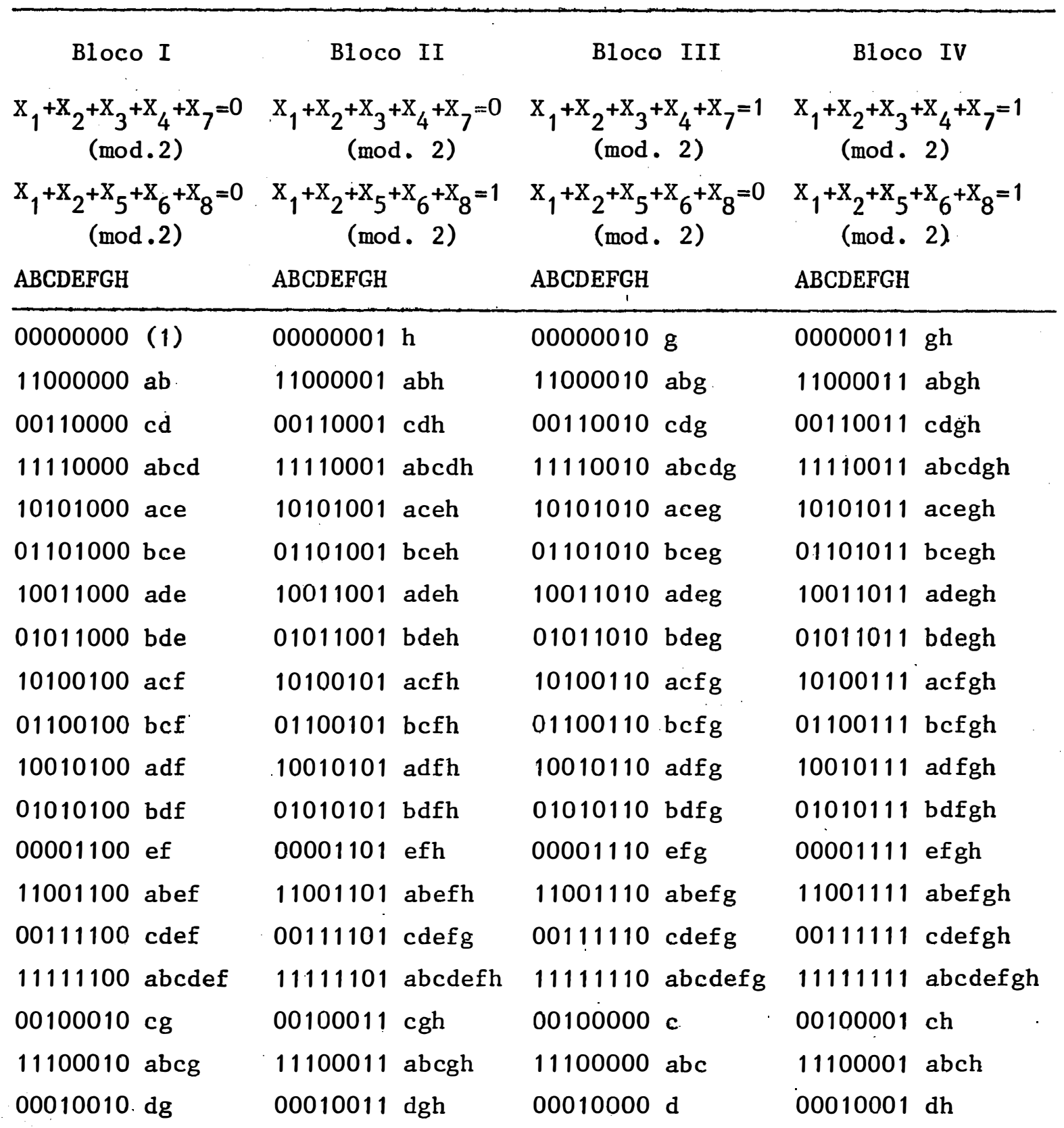




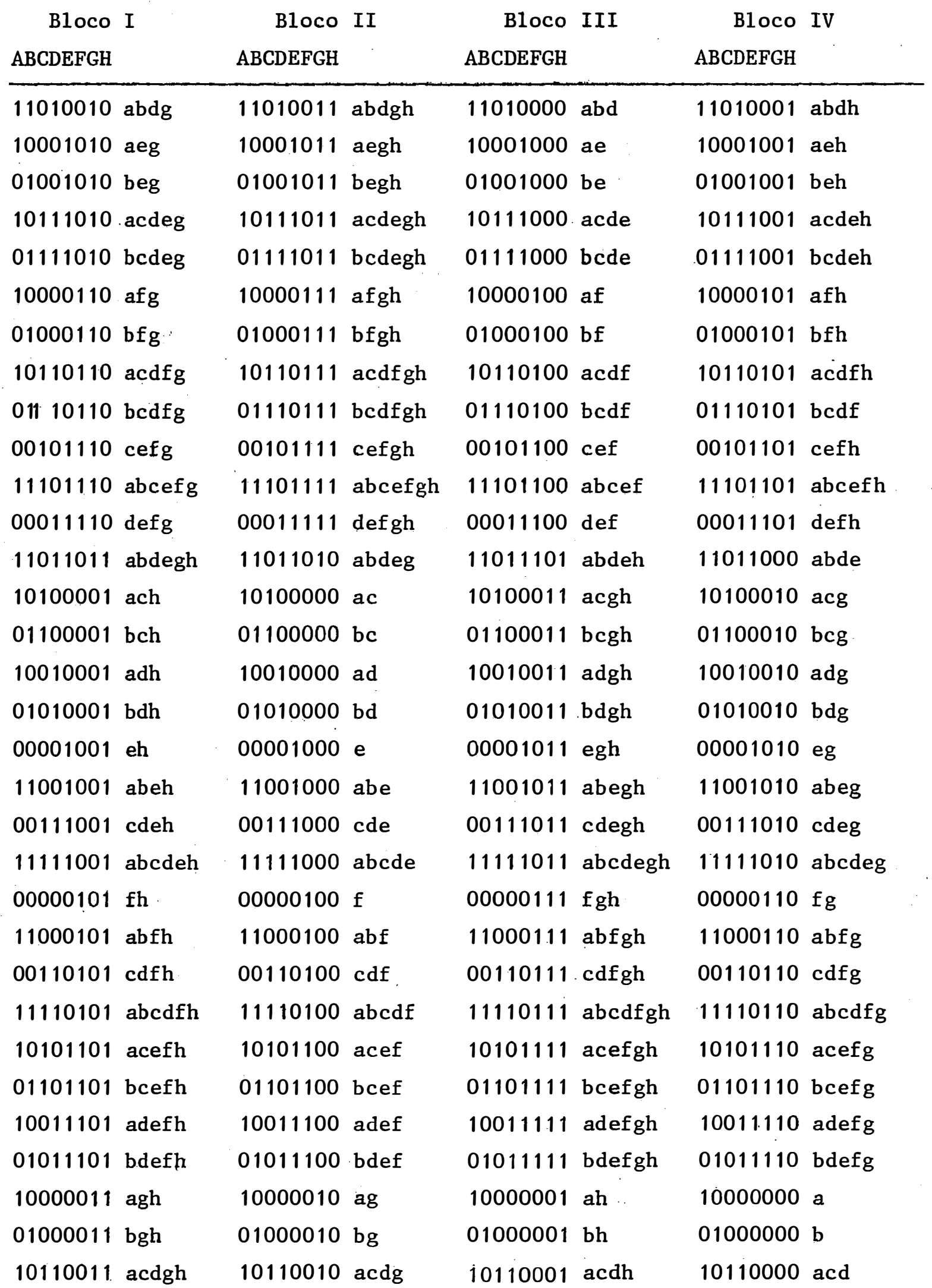




\begin{tabular}{llll}
$\begin{array}{c}\text { Bloco I } \\
\text { ABCDEFGH }\end{array}$ & $\begin{array}{c}\text { Bloco II } \\
\text { ABCDEFGH }\end{array}$ & $\begin{array}{c}\text { Bloco III } \\
\text { ABCDEFGH }\end{array}$ & $\begin{array}{c}\text { Bloco IV } \\
\text { ABCDEFGH }\end{array}$ \\
\hline 01110011 bedgh & $01110010 \mathrm{bedg}$ & $01110001 \mathrm{bcdh}$ & $01110000 \mathrm{bcd}$ \\
$00101011 \mathrm{cegh}$ & $00101010 \mathrm{ceg}$ & $00101001 \mathrm{ceh}$ & $00101000 \mathrm{ce}$ \\
11101011 abcegh & $11101010 \mathrm{abceg}$ & $11101001 \mathrm{abceh}$ & $11101000 \mathrm{abce}$ \\
00011011 degh & $00011010 \mathrm{deg}$ & $00011001 \mathrm{deh}$ & $00011000 \mathrm{de}$ \\
11011110 abdefg & $11011111 \mathrm{abdefgh}$ & $11011100 \mathrm{abdef}$ & $11011101 \mathrm{abdefh}$ \\
00100111 cfgh & $00100110 \mathrm{cfg}$ & $00100101 \mathrm{cfh}$ & $00100100 \mathrm{cf}$ \\
11100111 abcfgh & $11100110 \mathrm{abcfg}$ & $11100101 \mathrm{abcfh}$ & $11100100 \mathrm{abcf}$ \\
00010111 afgh & $00010110 \mathrm{dfg}$ & $00010101 \mathrm{dfh}$ & $00010100 \mathrm{df}$ \\
11010111 abdfgh & $11010110 \mathrm{abdfg}$ & $11010101 \mathrm{abdfh}$ & $11010100 \mathrm{abdf}$ \\
10001111 aefgh & $10001110 \mathrm{aefg}$ & $10001101 \mathrm{aefh}$ & $10001100 \mathrm{aef}$ \\
$01001111 \mathrm{befgh}$ & $01001110 \mathrm{befg}$ & $01001101 \mathrm{befh}$ & $01001100 \mathrm{bef}$ \\
10111111 acdefgh & $10111110 \mathrm{acdefg}$ & $10111101 \mathrm{acdefh}$ & $10111100 \mathrm{acdef}$ \\
$01111111 \mathrm{bcdefgh}$ & $01111110 \mathrm{bcdefg}$ & $01111101 \mathrm{bcdefh}$ & $01111100 \mathrm{bcdef}$
\end{tabular}

o bloco II é formado a partir do bloco I, adi cionando-se 1 a coluna correspondente ao fator $H$, ou multiplicando-se as combinações de tratamento por h. Da mesma forma são construidos os outros dois blocos, adicionando-se 1. da forma conveniente.

Tambèm poderiamos escrever o bloco I ou o blo co principal desta maneira: o bloco principal consiste dos pontos em que $x_{1} x_{2} x_{3} x_{4} x_{7}=-1$ e $x_{1} x_{2} x_{5} x_{6} x_{8}=-1$. Mu1tip1icando estas equações por $x_{7}$ e $x_{8}$, respectivamente, e notando que $x_{7}^{2}=x_{8}^{2}=1$, temos que:

$$
x_{7}=-x_{1} x_{2} x_{3} x_{4} \quad \text { e } \quad x_{8}=-x_{1} x_{2} x_{5} x_{6}
$$


Então podemos escrever o bloco principal com base no delineamento $2^{6}$ completo com os fatores $A, B, C, 1, D$, E e F, e então adicionamos os outros dois fatores G e H, colocando $x_{7}=x_{1}+x_{2}+x_{3}+x_{4}(\bmod .2)$ e $x_{8}=x_{1}+x_{2}+x_{5}+x_{6}(\bmod .2)$, ou $x_{7}=-x_{1} x_{2} x_{3} x_{4}$ e $x_{8}=-x_{1} x_{2} x_{5} x_{6}$. Analogamente, o segundo bloco seria obtido fazendo $x_{7}=-x_{1} x_{2} x_{3} x_{4}$ e $x_{8}=+x_{1} x_{2} x_{5} x_{6} ; 0$ terceiro bloco fazendo $x_{7}=+x_{1} x_{2} x_{3} x_{4}$ e $x_{8}=-x_{1} x_{2} x_{5} x_{6}$; e o quarto bloco fazendo $x_{7}=+x_{1} x_{2} x_{3} x_{4}$ e $x_{8}=+x_{1} x_{2} x_{5} x_{6}$.

\begin{tabular}{|c|c|c|c|c|c|c|c|c|c|}
\hline & $\begin{array}{l}\mathrm{A} \\
\mathrm{X}_{1} \\
\end{array}$ & $\begin{array}{l}\mathrm{B} \\
\mathrm{x}_{2} \\
\end{array}$ & $\begin{array}{r}\mathrm{C} \\
\mathrm{x}_{3} \\
\end{array}$ & $\begin{array}{l}\mathrm{D} \\
\mathrm{X} \\
\end{array}$ & $\begin{array}{l}E \\
X_{5}\end{array}$ & $\begin{array}{l}F \\
X_{6} \\
\end{array}$ & $\mathrm{x}_{7}=\mathrm{x}_{1}+\mathrm{x}_{2}+\mathrm{x}_{3}+\mathrm{x}_{4}$ & $\mathrm{X}_{8}=\mathrm{X}_{1}+\mathrm{X}_{2}+\mathrm{X}_{5}+\mathrm{X}_{6}$ & \\
\hline (1) & 0 & 0 & 0 & 0 & 0 & 0 & 0 & 0 & (1) \\
\hline $\mathbf{a}$ & 1. & 0 & 0 & 0 & 0 & 0 & 1 & 1 & agh \\
\hline b & 0 & 1 & 0 & 0 & 0 & 0 & 1 & 1 & bgh \\
\hline$a b$ & 1 & 1 & 0 & 0 & 0 & 0 & 0 & 0 & $a b$ \\
\hline c. & 0 & 0 & 1 & 0 & 0 & 0 & 1 & 0 & $\mathrm{cg}$ \\
\hline ac & 1 & 0 & 1 & 0 & 0 & 0 & 0 & 1 & ach \\
\hline bc & 0 & 1 & 1 & 0 & 0 & 0 & 0 & 1 & bch \\
\hline$a b c$ & 1 & 1 & 1 & 0 & 0 & 0 & 1 & 0 & $a b c g$ \\
\hline$d$ & 0 & 0 & 0 & 1 & 0 & 0 & 1 & 0 & $\mathrm{dg}$ \\
\hline ad & 1 & 0 & 0 & 1 & 0 & 0 & 0 & 1 & adh \\
\hline bd & 0 & 1 & 0 & 1 & 0 & 0 & 0 & 1 & bdh \\
\hline abd & 1 & 1 & 0 & 1 & 0 & 0 & 1 & 0 & abdg \\
\hline $\mathrm{cd}$ & 0 & 0 & 1 & 1 & 0 & 0 & 0 & 0 & $c d$ \\
\hline acd & 1 & 0 & 1 & 1 & 0 & 0 & 1. & 1 & acdgh \\
\hline bcd & 0 & 1 & 1 & 1 & 0 & 0 & 1 & 1 & bcdgh \\
\hline$a b c d$ & 1 & 1 & 1 & 1 & 0 & 0 & 0 & 0 & abcd \\
\hline e & 0 & 0 & 0 & 0 & 1 & 0 & 0 & 1 & eh \\
\hline ae & 1 & 0 & 0 & 0 & 1 & 0 & 1 & 0 & aeg \\
\hline be & 0 & 1 & 0 & 0 & 1 & 0 & 1 & 0 & beg \\
\hline
\end{tabular}




\begin{tabular}{|c|c|c|c|c|c|c|c|c|c|}
\hline & $\mathrm{A}$ & B & $\mathrm{C}$ & $\mathrm{D}$ & $\mathrm{E}$ & $\mathrm{F}$ & $G$ & $\mathrm{H}$ & \\
\hline abe & 1 & 1 & 0 & 0 & 1 & 0 & 0 & 1 & abeh \\
\hline ce & 0 & 0 & 1 & 0 & 1 & 0 & 1 & 1 & cegh \\
\hline ace & 1 & 0 & 1 & 0 & 1 & 0 & 0 & 0 & ace \\
\hline bce & 0 & 1 & 1 & 0 & 1 & 0 & 0 & 0 & bce \\
\hline abce & 1 & 1 & 1 & 0 & 1 & 0 & 1 & 1 & abcegh \\
\hline de & 0 & 0 & 0 & 1. & 1 & 0 & 1 & 1 & degh \\
\hline ade & 1 & 0 & 0 & 1 & 1 & 0 & 0 & 0 & ade \\
\hline bde & 0 & 1 & 0 & 1 & 1 & 0 & 0 & 0 & bde \\
\hline abde & 1 & 1 & 0 & 1 & 1 & 0 & 1 & 1 & abdegh \\
\hline cde & 0 & 0 & 1 & 1 & 1 & 0 & 0 & 1 & cdeh \\
\hline acde & 1 & 0 & 1 & 1 & 1 & 0 & 1 & 0 & acdeg \\
\hline bcde & 0 & 1 & 1 & 1 & 1 & 0 & 1 & 0 & bcdeg \\
\hline abcde & 1 & 1 & 1 & 1 & 1 & 0 & 0 & 1 & abcdeh \\
\hline$f$ & 0 & 0 & 0 & 0 & 0 & 1 & 0 & 1 & $\mathrm{fh}$ \\
\hline af & 1 & 0 & 0 & 0 & 0 & 1 & 1 & 0 & afg \\
\hline$b f$ & 0 & 1 & 0 & 0 & 0 & 1 & 1 & 0 & bfg \\
\hline$a b f$ & 1 & 1 & 0 & 0 & 0 & 1 & 0 & 1 & $a b f h$ \\
\hline cf & 0 & 0 & 1 & 0 & 0 & 1 & 1 & 1 & cfgh \\
\hline $\operatorname{acf}$ & 1 & 0 & 1 & 0 & 0 & 1 & 0 & 0 & $\operatorname{acf}$ \\
\hline bcf & 0 & 1 & 1 & 0 & 0 & 1 & 0 & 0 & bcf \\
\hline abcf & 1 & 1 & 1 & 0 & 0 & 1 & 1 & 1 & abcfgh \\
\hline $\mathrm{df}$ & 0 & 0 & 0 & 1 & 0 & 1 & 1 & 1 & $\mathrm{dfgh}$ \\
\hline $\operatorname{adf}$ & 1 & 0 & 0 & 1 & 0 & 1 & 0 & 0 & adf \\
\hline$b d f$ & 0 & 1 & 0 & 1 & 0 & 1 & 0 & 0 & bdf \\
\hline abdf & 1 & 1 & 0 & 1 & 0 & 1 & 1 & 1 & abdfgh \\
\hline $\operatorname{cdf}$ & 0 & 0 & 1 & 1 & 0 & 1 & 0 & 1 & $c d f h$ \\
\hline $\operatorname{acd} f$ & $i 1$ & 0 & 1 & 1 & 0 & 1 & 1 & 0 & $\operatorname{acdfg}$ \\
\hline bcdf & 0 & 1 & 1 & 1 & 0 & 1 & 1 & 0 & bcdfg \\
\hline$a b c d f$ & 1 & 1 & 1 & 1 & 0 & 1 & 0 & 1 & abcdfh \\
\hline ef & 0 & 0 & 0 & 0 & 1 & 1 & 0 & 0 & ef \\
\hline aef & 1 & 0 & 0 & 0 & 1 & 1 & 1 & 1 & aefgh \\
\hline bef & 0 & 1 & 0 & 0 & 1 & 1 & 1 & 1 & befgh \\
\hline
\end{tabular}




\begin{tabular}{lllllllllll}
\hline & A & B & C & D & E & F & G & H & \\
\hline abef & 1 & 1 & 0 & 0 & 1 & 1 & 0 & 0 & abef \\
cef & 0 & 0 & 1 & 0 & 1 & 1 & 1 & 0 & cefg \\
acef & 1 & 0 & 1 & 0 & 1 & 1 & 0 & 1 & acefh \\
bcef & 0 & 1 & 1 & 0 & 1 & 1 & 0 & 1 & bcefh \\
abcef & 1 & 1 & 1 & 0 & 1 & 1 & 1 & 0 & abcefg \\
def & 0 & 0 & 0 & 1 & 1 & 1 & 1 & 0 & defg \\
adef & 1 & 0 & 0 & 1 & 1 & 1 & 0 & 1 & adefh \\
bdef & 0 & 1 & 0 & 1 & 1 & 1 & 0 & 1 & bdefh \\
abdef & 1 & 1 & 0 & 1 & 1 & 1 & 1 & 0 & abdefg \\
cdef & 0 & 0 & 1 & 1 & 1 & 1 & 0 & 0 & cdef \\
acdef & 1 & 0 & 1 & 1 & 1 & 1 & 1 & 1 & acdefgh \\
bcdef & 0 & 1 & 1 & 1 & 1 & 1 & 1 & 1 & bcdefgh \\
abcdef & 1 & 1 & 1 & 1 & 1 & 1 & 0 & 0 & abcdef \\
\hline
\end{tabular}

0 termo fracāo regular $2^{n-k}$, è usado para des crever somente aqueles conjuntos $\operatorname{com} 2^{n-k}$ combinações de tra tamentos, que poderiam ocorrer no mesmo bloco, quando o fato rial completo é dividido em $2^{k}$ blocos, confundindo exatamente $2^{k}-1$ efeitos. Portanto, podemos definir uma fração $2^{n-k}$, como um conjunto com $2^{n-k}$ pontos, que constituem um subgrupo de combinações de tratamentos.

No delineamento $2^{8}$ descrito acima, o bloco principal è a fração definida por:

$$
I=-A B C D G=-A B E F H=+C D E F G H
$$


As outras frações são definidas por

$$
\begin{aligned}
& I=-\mathrm{ABCDG}=+\mathrm{ABEFH}=-\mathrm{CDEFGH} \\
& I=+\mathrm{ABCDG}=-\mathrm{ABEFH}=-\mathrm{CDEFGH} \\
& I=+\mathrm{ABCDG}=+\mathrm{ABEFH}=+\mathrm{CDEFGH}
\end{aligned}
$$

O termo fracão principal è usado para indicar a fração que contëm o tratamento controle (1), por analogia com o bloco principal.

No exemplo acima, para enfocar o conjunto de aliases, vamos tomar a fração referente a seguinte relação

\begin{tabular}{|c|c|c|c|c|c|}
\hline $\begin{array}{l}\text { Efeitos } \\
\text { Principais }\end{array}$ & Alias & $\begin{array}{l}\text { Interaçoes' de } \\
2 \text { fatores }\end{array}$ & Alias & $\begin{array}{c}\text { Interaçōes de } \\
3 \text { fatores. }\end{array}$ & Alias \\
\hline A & $\mathrm{BCDG}, \mathrm{BEFH}, \mathrm{ACDEFGH}$ & $\mathrm{AB}$ & $\mathrm{CDG}, \mathrm{EFH}, \mathrm{ABCDEFGH}$ & $\mathrm{ACE}$ & BDEG, BCFH, ADFGH \\
\hline B & ACDG, AEFH, BCDEFGH & $\mathrm{AC}$ & BDG, BCEFH , ADEFGH & ACF & $\mathrm{BDFG}, \mathrm{BCEH}, \mathrm{ADEGH}$ \\
\hline C & $\mathrm{ABDG}, \mathrm{ABCEFH}, \mathrm{DEFGH}$ & $\mathrm{AD}$ & $\mathrm{BCG}, \mathrm{BDEFH}, \mathrm{ACEFGH}$ & $\mathrm{ACH}$ & $\mathrm{BDGH}, \mathrm{BCEF}, \mathrm{ADEFG}$ \\
\hline D & $\mathrm{ABCG}, \mathrm{ABDEFH}, \mathrm{CEFGH}$ & AG & $\mathrm{BCDEG}, \mathrm{BFH}, \mathrm{ACDFGH}$ & $\mathrm{ADE}$ & $\mathrm{BCEG}, \mathrm{BDFH}, \mathrm{ACFGH}$ \\
\hline E & ABCDEG, $\mathrm{ABFH}, \mathrm{CDFGH}$ & $\mathrm{AF}$ & $\mathrm{BCDFG}, \mathrm{BEH}, \mathrm{ACDEGH}$ & $\mathrm{ADF}$ & BCFG, BDEH, ACEGH \\
\hline F & $\mathrm{ABCDFG}, \mathrm{ABEH}, \mathrm{CDEGH}$ & AG & $\mathrm{BCD}, \mathrm{BEFGH}, \mathrm{ACDEFH}$ & $\mathrm{ADH}$ & $\mathrm{BCGH}, \mathrm{BDEF}, \mathrm{ACEFG}$ \\
\hline G & $\mathrm{ABCD}, \mathrm{ABEFGH}, \mathrm{CDEFH}$ & $\mathrm{AH}$ & $\mathrm{BCDGH}, \mathrm{BEF}, \mathrm{ACDEFG}$ & AEG & $\mathrm{BCDE}, \mathrm{BFGH}, \mathrm{ACDFH}$ \\
\hline \multirow[t]{12}{*}{ H } & $\mathrm{ABCDGH}, \mathrm{ABEF}, \mathrm{CDEFG}$ & BC & $\mathrm{ADG}, \mathrm{ACEFH}, \mathrm{BDEFGH}$ & AFG & $\mathrm{BCDF}, \mathrm{BEGH}, \mathrm{ACDEH}$ \\
\hline & & $\mathrm{BD}$ & $\mathrm{ACG}, \mathrm{ADEFH}, \mathrm{BCEFGH}$ & AGH & $\mathrm{BCDH}, \mathrm{BEFG}, \mathrm{ACDEF}$ \\
\hline & - & $\mathrm{BE}$ & $\mathrm{ACDEG}, \mathrm{AFH}, \mathrm{BCDFGH}$ & BCE & $\mathrm{ADEG}, \mathrm{ACFH}, \mathrm{BDFGH}$ \\
\hline & & $\mathrm{BF}$ & $\mathrm{ACDFG}, \mathrm{AGH}, \mathrm{BCDEGH}$ & BCF & $\mathrm{ADFG}, \mathrm{ACEH}, \mathrm{BDEGH}$ \\
\hline & & BG & $\mathrm{ACD}, \mathrm{AEFGH}, \mathrm{BCDEFH}$ & $\mathrm{BCH}$ & $\mathrm{ADGH}, \mathrm{ACEF}, \mathrm{BDEFG}$ \\
\hline & & $\mathrm{BH}$ & $\mathrm{ACDGH}, \mathrm{AEF}, \mathrm{BCDEFG}$ & $\mathrm{BDE}$ & $\mathrm{ACEG}, \mathrm{ADFH}, \mathrm{BCFGH}$ \\
\hline & & $C D$ & $\mathrm{ABG}, \mathrm{ABCDEFH}, \mathrm{EFGH}$ & $\mathrm{BDF}$ & $\mathrm{ACFG}, \mathrm{ADEH}, \mathrm{BCEGH}$ \\
\hline & & $\mathrm{CE}$ & $\mathrm{ABDEG}, \mathrm{ABCFH}, \mathrm{DFGH}$ & $\mathrm{BDH}$ & $\mathrm{ACGH}, \mathrm{ADEF}, \mathrm{BCEFG}$ \\
\hline & & CF & $\mathrm{ABDFG}, \mathrm{ABCEH}, \mathrm{DEGH}$ & BEG & $\mathrm{ACDE}, \mathrm{AFGH}, \mathrm{BCDFH}$ \\
\hline & & CG & $\mathrm{ABD}, \mathrm{ABCEFGH}, \mathrm{DEFH}$ & BFG & $\mathrm{ACDF}, \mathrm{AEGH}, \mathrm{BCDEH}$ \\
\hline & & $\mathrm{CH}$ & $\mathrm{ABDGH}, \mathrm{ABCEF}, \mathrm{DEFG}$ & BGH & $\mathrm{ACDH}, \mathrm{AEFG}, \mathrm{BCDEF}$ \\
\hline & & $\mathrm{DE}$ & $\mathrm{ABCEG}, \mathrm{ABDFH}, \mathrm{CFGH}$ & $\mathrm{CDE}$ & $\mathrm{ABEG}, \mathrm{ABCDFG}, \mathrm{FGH}$ \\
\hline
\end{tabular}
de identidade:

$$
I=+A B C D G=+A B E F H=+C D E F G H
$$




\begin{tabular}{|c|c|c|c|c|}
\hline & $\begin{array}{l}\text { Interaçōes de } \\
2 \text { fatores }\end{array}$ & Alias & $\begin{array}{l}\text { Interações de } \\
3 \text { fatores }\end{array}$ & Alias \\
\hline & $\mathrm{DF}$ & $\mathrm{ABCFG}, \mathrm{ABDEH}, \mathrm{CEGH}$ & $\mathrm{CDF}$ & $\mathrm{ABFG}, \mathrm{ABCDEH}, \mathrm{EGH}$ \\
\hline & DG & $\mathrm{ABC}, \mathrm{ABDEFGH}, \mathrm{CEFH}$ & $\mathrm{CDH}$ & $\mathrm{ABGH}, \mathrm{ABCDEF}, \mathrm{EFG}$ \\
\hline & $\mathrm{DH}$ & $\mathrm{ABCGH}, \mathrm{ABDEF}, \mathrm{CEFG}$ & CEF & $\mathrm{ABDEFG}, \mathrm{ABCH}, \mathrm{DGH}$ \\
\hline & $\mathrm{EF}$ & $\mathrm{ABCDEFG}, \mathrm{ABH}, \mathrm{CDEH}$ & CEG & $\mathrm{ABDE}, \mathrm{ABCFGH}, \mathrm{DFH}$ \\
\hline & EG & $\mathrm{ABCDE}, \mathrm{ABFGH}, \mathrm{CDFH}$ & $\mathrm{CEH}$ & $\mathrm{ABDEGH}, \mathrm{ABCF}, \mathrm{DFG}$ \\
\hline & EH & $\mathrm{ABCDEGH}, \mathrm{ABF}, \mathrm{CDFG}$ & CFG & $\mathrm{ABDF}, \mathrm{ABCEGH}, \mathrm{DEH}$ \\
\hline & FG & $\mathrm{ABCDF}, \mathrm{ABEGH}, \mathrm{CDEH}$ & $\mathrm{CFH}$ & $\mathrm{ABDFGH}, \mathrm{ABCE}, \mathrm{DEG}$ \\
\hline & FH & $\mathrm{ABCDFGH}, \mathrm{ABE}, \mathrm{CDEG}$ & CGH & $\mathrm{ABDH}, \mathrm{ABCEFG}, \mathrm{DEF}$ \\
\hline & GH & $\mathrm{ABCDH}, \mathrm{ABEFG}, \mathrm{CDEF}$ & & \\
\hline
\end{tabular}

Se pudermos considerar as interações de 3 ou mais fatores como insignificante, os efeitios principais e as interações de 2 fatores são estimáveis. E para a estimativa do erro temos 27 graus de liberdade. Assim o esquema do qua dro da anālise de variāncia será:

C. variação G.L.

Efeitos principais

Interações de 2 fatores 28

$\begin{array}{ll}\text { Residuo } & 27\end{array}$

Tota 1

63

Como vimos nos dois exemplos, a metade do fatorial $2^{6}$ e $1 / 4$ do fatorial $2^{8}$ estavam em delineamento inte $\underline{i}$ ramente ao acaso. No próximo item vamos colocar estes dois exemplos em blocos ao acaso. 


\subsection{Fatoriais fracionados da série $2^{n}$ em blocos ao acaso}

\section{EXEMPLO 1.}

Vamos tomar a metade do fatorial $2^{6}$. Se o pesquisador estivesse interessado em colocar essa metade em dois blocos de 16 combinações de tratamentos, ele teria que dispor de uma interaça com sua respectiva alias para confun dir comblocos. Vamos tomar a metade referente a relaça de identidade $I=+A B C D E F$.

Para escolher a interação para ser confundida, o pesquisador deve ter em mente que a interação generalizada desta, com a relação de identidade, tambèm será confundida.

Como estamos supondo que as interações de 3 ou mais fatores são insignificantes, então vamos escolher a interação $A E F=B C D$. Assim, temos os seguintes blocos: 


\section{BLOCO I}

BLOCO II

B C D

$\mathrm{x}_{2}+\mathrm{x}_{3}+\mathrm{x}_{4}=1$

$\mathrm{x}_{2}+\mathrm{x}_{3}+\mathrm{x}_{4}=0$

$110000 \mathrm{ab}$

$000101 \mathrm{df}$

100010 ae

110110 abde

101000 ac

$111100 \mathrm{abcd}$

100001 af

$110101 \mathrm{abdf}$

100100 ad

110011 abef

$011001 \mathrm{bc}$

101110 acde

010010 be

101011 acef

$010101 \mathrm{bd}$

101101 acdf

$010001 \mathrm{bf}$

100111 adef

$001100 \mathrm{~cd}$

011011 bcef

001010 ce

011110 bcde

000011 ef

010111 bdef

001001 cf

$011101 \mathrm{bcdf}$

111010 abce 001111 cdef

111111 abcdef

Neste caso o esquema do quadro da anālise de variância serā:

C. Variação

G.L.

Blocos

1

Efeitos principais

6

Interações de 2 fatores

15

Residuo

9

Tota 1 


\section{EXEMPLO 2 .}

Vamos tomar $1 / 4$ do fatorial $2^{8}$. Se o pesquisador estivesse interessado em colocar este $1 / 4$ em 1" quatro blocos com 16 combinações de tratamentos, ele teria que dispor de duas interaçoes com suas respectivas alias para confundir com blocos. Essas interações devem ser escolhidas de tal maneira que, o produto delas com as interações presentes na relação de identidade não sejam efeitos principais e nem interações de 2 fatores. Também devemos observar que o produto entre a interação generalizada das duas interaçós esco lhidas com aquelas presentes na relaça de identifidade não resulta em efeitos principais ou interaços de dois fatores.

Vamos tomar a quarta fração referente a relação de identidade $I=+A B C D G=+A B E F H=+C D E F G H$.

Se escolhermos para confundir comblocos as interações ACEGH, BDEFG, então a sua interação generalizada é ABCDFH. Assim, com esta escolha também serão confundidas as seguintes interações:

$$
\begin{aligned}
& \mathrm{ACEGH}=\mathrm{BDEH}=\mathrm{BCFG}=\mathrm{ADF} \\
& \mathrm{BDEFG}=\mathrm{ACEF}=\mathrm{ADGH}=\mathrm{BCH} \\
& \mathrm{ABCDFH}=\mathrm{FGH}=\mathrm{CDE}=\mathrm{ABEG}
\end{aligned}
$$

Logo, vemos que nesse caso não confundimos efeitos principais nem interaçóes de 2 fatores, e os 4,blocos são dados como segue: 


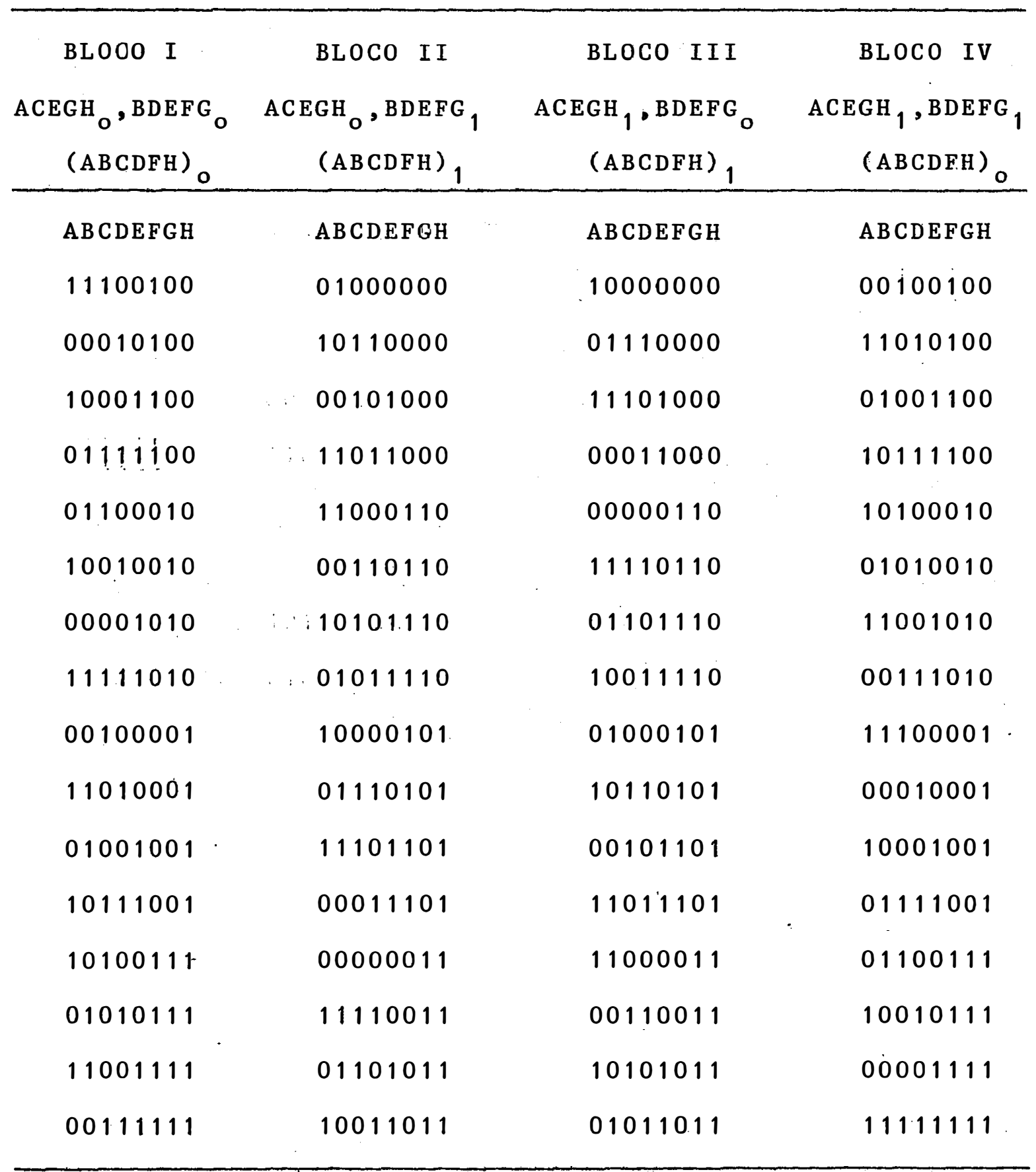

Como o experimento escolhido não contém o tratamento controle (1) entre as combinacooes de tratamentos, nenhum dos quatro blocos é o subgrupo intrabloco. Mesmo as- 
sim, podemos obter os outros a partir do primeiro jä l.construido.

Construimos o primeiro bloco procurando no ex perimento selecionado, as combinaçoes de tratamentos que satisfazem as equações, $x_{1}+x_{3}+x_{5}+x_{7}+x_{8}=0(\bmod \cdot 2)$ e $\mathrm{x}_{2}+\mathrm{X}_{4}+\mathrm{X}_{5}+\mathrm{X}_{6}+\mathrm{X}_{7}=0(\bmod .2)$, e depois colocamos estas combinações de tratamentos na "ordem padrão".

Para construirmos o segundo bloco escolhemos uma das combinações de tratamentos que satisfazem as equacões, $x_{1}+x_{3}+x_{5}+x_{7}+x_{8}=0(\bmod .2)$ e $x_{2}+x_{4}+x_{5}+x_{6}+x_{7}=1(\bmod$. 2), por exemplo, $\underline{b}=01000000$, em seguida verificamos qual é o número modular que somado à primeira combinacão de tratamento do bloco I, nos da essa combinação de tratamento. Nes se caso, o número modular é 10100100. Fieito isso, somamos este número a todas combinações de tratamentos do bloco I ob tendo-se o bloco II.

Para obtermos os outros blocos o procedimento é anälogo. Assim, para obtermos o bloco III, inicialmente es colhemos $\underline{a}=10000000$ para ser sua primeira combinaça de tratamento, e o número modular é 01100100 . A primeira comb nação de tratamento escolhida para o abloco l IV foi $\underline{c f}=00100100$, e o número modular que devemos somar as combinações de tratamentos do bloco I é: 11000000 .

Neste caso, o esquema do quadro da anālise de 
variāncia serā:

C. variação

G.L.

Blocos

3

Efeitos principais

8

Interações de 2 fatores

28

Residuo

.24

Tota 1

63

\subsubsection{FATORIAL FRACIONADO NO SISTEMA $3^{\text {n }}$}

o fatorial fracionado tem. sido utilizado com bastante sucessonos, experimentos fiatoriais da sërie $2^{n}$, sen do também.ütil em experimentos fatoriais da série $3^{n}$, em menor escala devido a dificuldade de construção.

Com essa idéia, vamos examinar as circunstāncias em que poderiamos considerar o fatorial fracionado na sërie $3^{n}$ como um delineamento de considerāvel valor prático. Se o material experimental é constituido de tal maneira, que as interações envolvendo 3 ou mais fatores sejam insignifi cantes, nós podemos usar o fatorial fracionado $3^{n}, \therefore$ achando uma relação de identidade, tal que os efeitos principais e interações de 2 fatores sejam estimäveis, nou seja, as suas aliases sejam interações de 3 ou mais fatores. 
Vamos iniciar o estudo do fatorial fracionado na série $3^{n}$, com um exemplo simples para enfocar os problemas de construção desse experimento, ou seja, $1 / 3$ da repetição do fatorial $3^{3}$. Antes de iniciar este estudo, devemos lembrar que em um fatorial $3^{3}$, os 26 graus de 1 iberdade para tratamentos podem ser divididos em 13 pares de graus de 1 iberdade como segue:

- Efeitos Principais: A, B, C

- Interações de 2 fatores: $\mathrm{AB}, \mathrm{AB}^{2}, \mathrm{AC}, \mathrm{AC}^{2}, \mathrm{BC} \mathrm{e} \mathrm{BC}^{2}$

- Interações de 3 fatores: $\mathrm{ABC}, \mathrm{ABC}^{2}, \mathrm{AB}^{2} \mathrm{C}$ e $\mathrm{AB}^{2} \mathrm{C}^{2}$

Para obtermos $1 / 3$ da repetição do fatorial $3^{3}$ è natural escolhermos uma interação de 3 fatores, ou seja, $A B C$ como a relação de identidade, i.è., I = ABC. Assim com base nessa relação de identidade nös podemos gerar os conjun tos de aliases, ou seja,

$$
\begin{aligned}
& A=A B^{2} C^{2}=B C \\
& B=A B^{2} C=A C \\
& A B=A B C^{2}=C \\
& A B^{2}=A C^{2}=B C^{2}
\end{aligned}
$$

da seguinte maneira:

$$
A=A \times A B C=A^{2} B C=\left(A^{2} B C\right)^{2}=A B^{2} C^{2}
$$

e

$$
\begin{aligned}
& A=A \times(A B C)^{2}=A \times A^{2} B^{2} C^{2}=A^{3} B^{2} C^{2}=\left(B^{2} C^{2}\right)^{2}=B C \\
& A=A B^{2} C^{2}=B C
\end{aligned}
$$


e assim por diante.

Como vemos, os efeitos principais A, B e C es tão completamente confundidos com interações de 2 fatores, isto"significa que estes efeitos principais só serão estimados se as interaçõs de 2 fatores forem consideradas como in significantes.

Sabemos que o fatorial $3^{3}$ tem 27 combinações de tratamentos, ou seja:
(1) $=000$
$c=001$
$c^{2}=002$
$a=100$
$\mathrm{ac}=10$ ?
$\mathrm{ac}^{2}=102$
$a^{2}=200$
$a^{2} c=201$
$\mathrm{a}^{2} \mathrm{c}^{2}=202$
$\mathrm{b}=010$
$b c=011$
$\mathrm{bc}^{2}=012$
$\mathrm{ab}=110$
$a b c=111$
$a b c^{2}=112$
$a^{2} b=210$
$\mathrm{a}^{2} \mathrm{bc}=211$
$\mathrm{a}^{2} \mathrm{bc}^{2}=212$
$b^{2}=020$
$b^{2} c=021$
$\mathrm{b}^{2} \mathrm{c}^{2}=022$
$a b^{2}=120$
$\mathrm{ab}^{2} \mathrm{c}=121$
$a b^{2} c^{2}-122$
$a^{2} b^{2}=220$
$a^{2} b^{2} c=221$
$a^{2} b^{2} c^{2}=222$

Com a relação de identidade $I=A B C$, temos as 3 frações seguintes: 
Fração 1

$\mathrm{ABC}_{0}$
Fração 2

$\mathrm{ABC}$
Fraça 3

$\mathrm{ABC}_{2}$

$x_{1}+x_{2}+x_{3}=0(\bmod .3) \quad x_{1}+x_{2}+x_{3}=1(\bmod .3) \quad x_{1}+x_{2}+x_{3}=2(\bmod .3)$

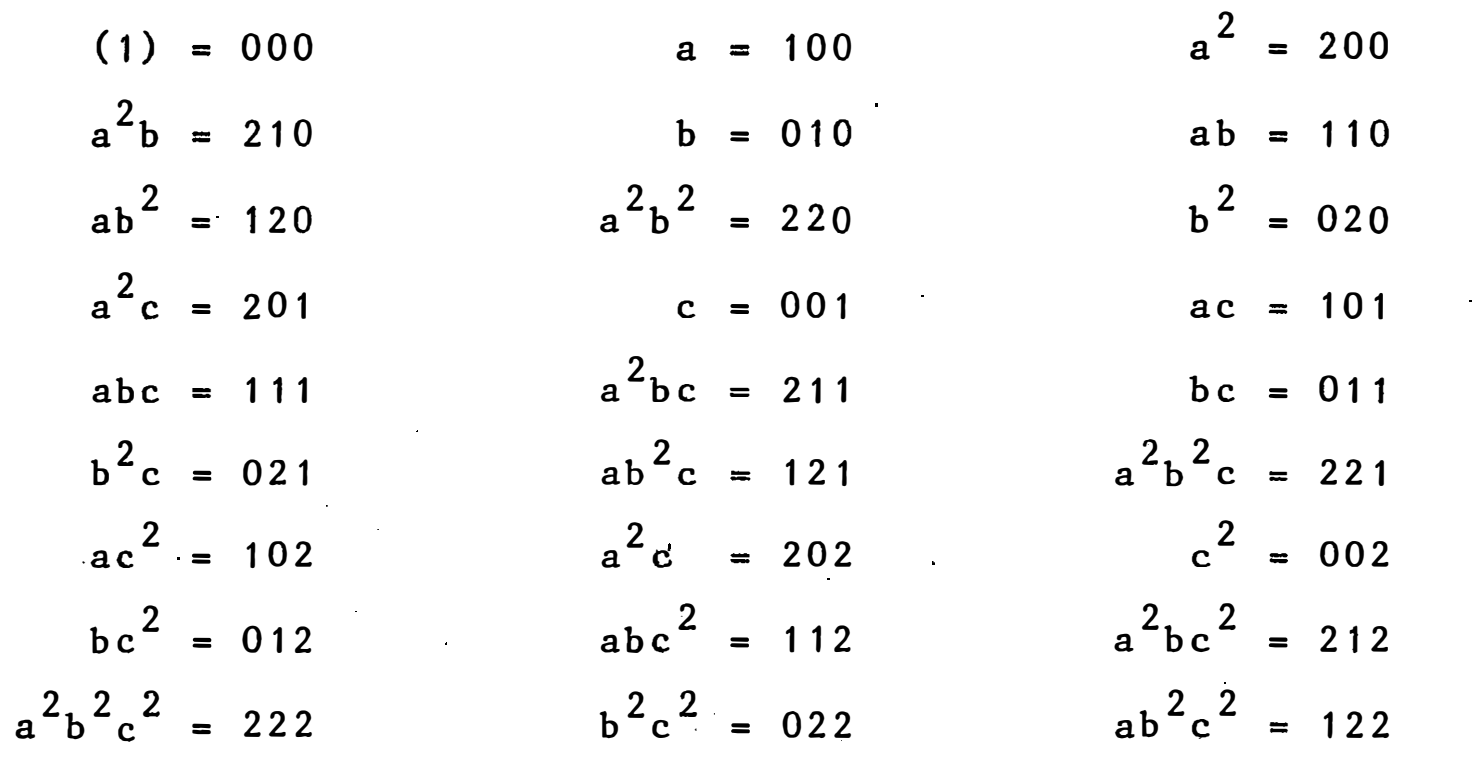

A fração 1 é o bloco principal ou o subgrupo intrabloco.

Agora daremos dois exemplos de fatoriais fra cionados da sërie $3^{n}$, onde os efeitos principais e as intera ções de 2 fatores são estimäveis. Nestes exemplos descrevemos os blocos principais e os conjuntos de aliases dos 2 experimentos.

\section{EXEMPLO 1.}

Vamos tomar $1 / 3$ do fatorial $3^{5}$. Assim, temos a fração $3^{5-1}$. Sejam A, B, C, D e F os cinco fatores representados por $x_{1}, x_{2}, x_{3}, x_{4}$ e $x_{5}$ respectivamente, e vamos es 
collher a seguinte relação de identidade $I=\mathrm{AB}^{2} \mathrm{CDE}^{2}$. Assim, temos as 3 frações seguintes:

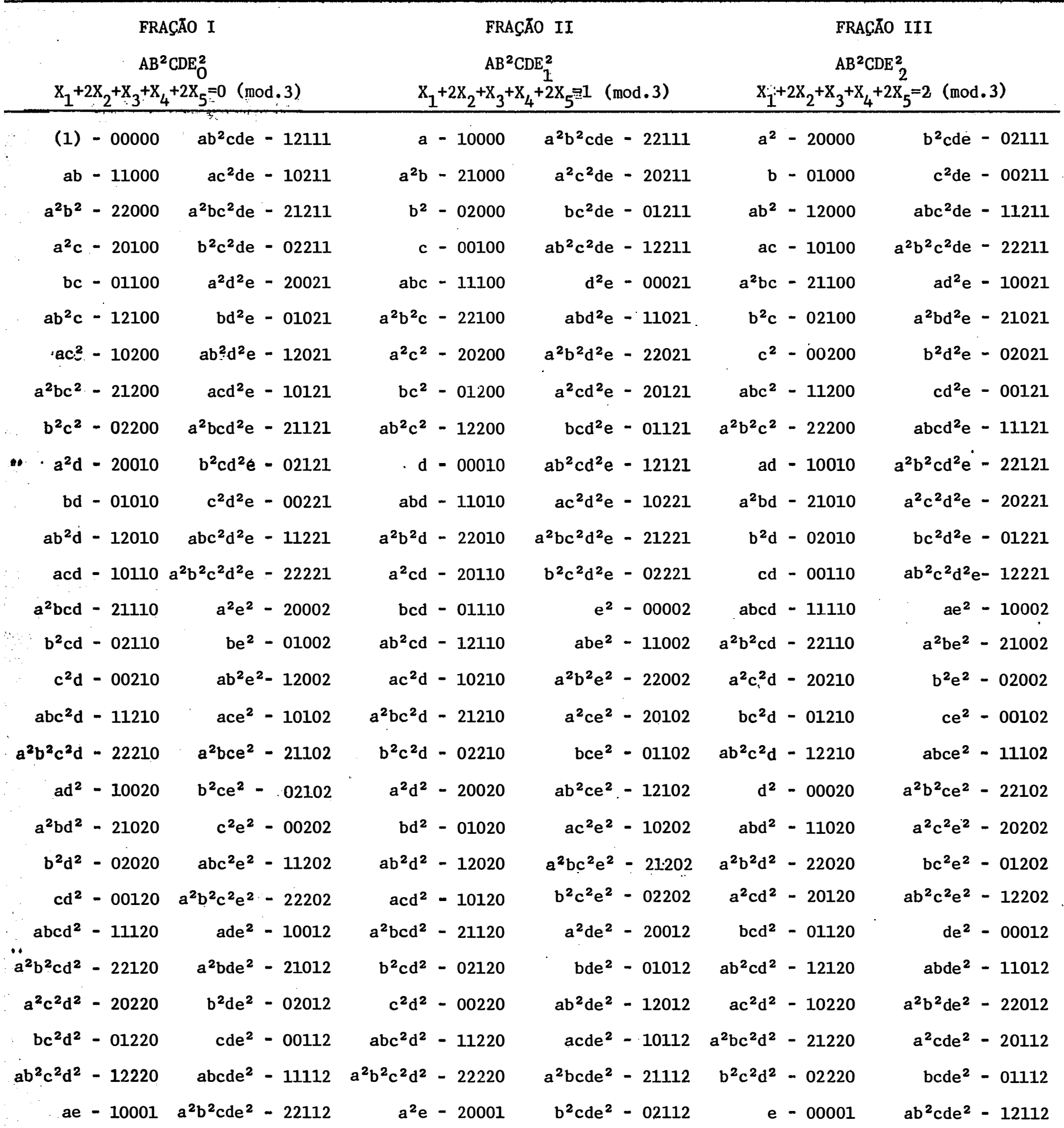




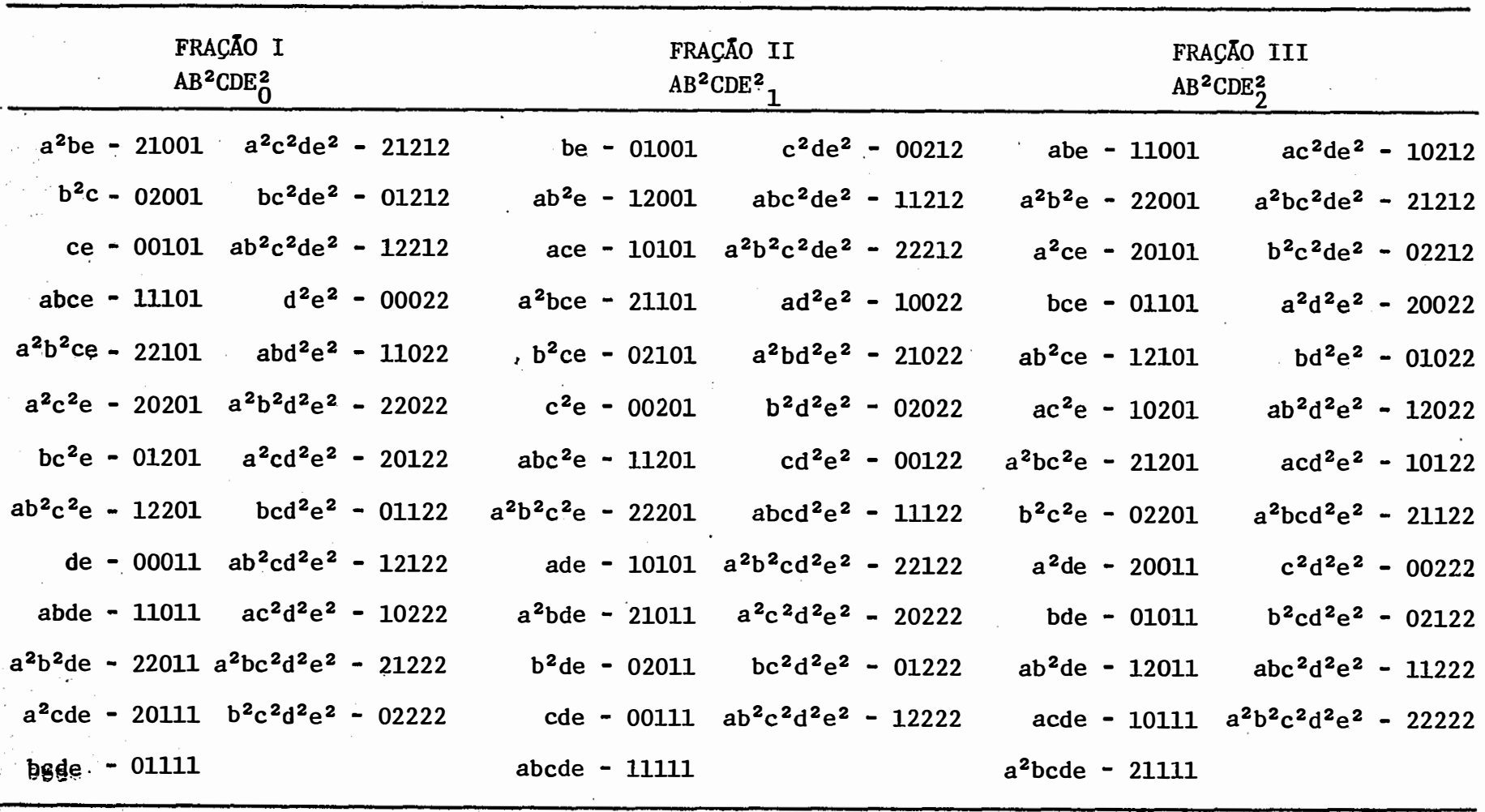

Neste caso, os conjuntos de aliases são : como segue: 
- Relação de Identidade : $I=\mathrm{AB}^{2} \mathrm{CDE}^{2}$

\begin{tabular}{|c|c|c|c|c|c|}
\hline $\begin{array}{l}\text { Efeitos } \\
\text { principais }\end{array}$ & A1ias & $\begin{array}{l}\text { Interações } \\
\text { de } 2 \text { fatores }\end{array}$ & Alias & $\begin{array}{l}\text { Interações } \\
\text { de } 3 \text { fatores }\end{array}$ & Alias \\
\hline A & $A B C^{2} D^{2} E ; B C^{2} D^{2} E$ & $\mathrm{AB}$ & $\mathrm{AC}^{2} \mathrm{D}^{2} \mathrm{E} ; \mathrm{BCDE}^{2}$ & $\mathrm{ABC}$ & $\mathrm{ACD}^{2} \mathrm{E} ; \mathrm{BDE}^{2}$ \\
\hline B & $\mathrm{ACDE}^{2} ; \mathrm{ABCDE}^{2}$ & $\mathrm{AB}^{2}$ & $\mathrm{AB}^{2} \mathrm{C}^{2} \mathrm{D}^{2} \mathrm{E} ; \mathrm{CDE}^{2}$ & $\mathrm{ABC}^{2}$ & $\mathrm{AD}^{2} \mathrm{E} ; \mathrm{BC}^{2} \mathrm{DE}^{2}$ \\
\hline C & $\mathrm{AB}^{2} \mathrm{C}^{2} \mathrm{DE}^{2} ; \mathrm{AB}^{2} \mathrm{DE}^{2}$ & AC & $\mathrm{ABCD}^{2} \mathrm{E} ; \mathrm{BD}^{2} \mathrm{E}$ & $\mathrm{AB}^{2} \mathrm{C}^{2}$ & $\mathrm{AB}^{2} \mathrm{D}^{2} \mathrm{E} ; \mathrm{CD}^{2} \mathrm{E}$ \\
\hline D & $A B^{2} C^{2} D^{2} E ; A B^{2} C E$ & $\mathrm{AC}^{2}$ & $\mathrm{ABD}^{2} \mathrm{E} ; \mathrm{BCD}^{2} \mathrm{E}$ & $\mathrm{ABD}$ & $\mathrm{AC}^{2} \mathrm{DE} ; \mathrm{BCE}^{2}$ \\
\hline \multirow[t]{15}{*}{ E } & $\mathrm{AB}^{2} \mathrm{CD} ; \mathrm{AB}^{2} \mathrm{CDE}$ & $\mathrm{AD}$ & $\mathrm{ABC}^{2} \mathrm{DE} ; \mathrm{BC}^{2} \mathrm{E}$ & $\mathrm{BCD}$ & $A C^{2} D^{2} E^{2} ; A B E^{2}$ \\
\hline & : & $A D^{2}$ & $A B C^{2} E ; B C^{2} \mathrm{DE}$ & $\mathrm{AC}^{2} \mathrm{D}$ & $\mathrm{ABDE} ; \mathrm{BCE}$ \\
\hline & & $\mathrm{AE}$ & $A B C^{2} \mathrm{D}^{2} ; \mathrm{BC}^{2} \mathrm{D}^{2} \mathrm{E}^{2}$ & $\mathrm{BC}^{2} \mathrm{D}$ & $\mathrm{AD}^{2} \mathrm{E}^{2} ; \mathrm{ABC}^{2} \mathrm{E}^{2}$ \\
\hline & & $\mathrm{AE}^{2}$ & $\mathrm{ABC}^{2} \mathrm{D}^{2} \mathrm{E}^{2} ; \mathrm{BC}^{2} \mathrm{D}^{2}$ & $\mathrm{ABD}^{2}$ & $\mathrm{AC}^{2} \mathrm{E} ; \mathrm{BCD}^{2} \mathrm{E}^{2}$ \\
\hline & & $\mathrm{BC}$ & $\mathrm{AC}^{2} \mathrm{DE}^{2} ; \mathrm{ABDE}^{2}$ & $A B^{2} D^{2}$ & $\mathrm{AB}^{2} \mathrm{C}^{2} \mathrm{E} ; \mathrm{CD}^{2} \mathrm{E}^{2}$ \\
\hline & & $\mathrm{BC}^{2}$ & $\mathrm{ADE}^{2} ; \mathrm{ABC}^{2} \mathrm{DE}^{2}$ & $\mathrm{ACD}^{2}$ & $\mathrm{ABCE} ; \mathrm{BDE}$ \\
\hline & & BD & $\mathrm{ACD}^{2} \mathrm{E}^{2} ; \mathrm{ABCE}^{2}$ & $\mathrm{BCD}^{2}$ & $\mathrm{AC}^{2} \mathrm{E}^{2} ; \mathrm{ABD}^{2} \mathrm{E}^{2}$ \\
\hline & & $\mathrm{BD}^{2}$ & $\mathrm{ACE}^{2} ; \mathrm{ABCD}^{2} \mathrm{E}^{2}$ & $\mathrm{AC}^{2} \mathrm{D}^{2}$ & $\mathrm{ABE} ; \mathrm{BCDE}$ \\
\hline & & $\mathrm{BE}$ & $\mathrm{ACD} ; \mathrm{ABCDE}$ & $\mathrm{AB}^{2} \mathrm{E}$ & $\mathrm{AB}^{2} \mathrm{C}^{2} \mathrm{D}^{2} ; \mathrm{CDE}$ \\
\hline & & $\mathrm{BE}^{2}$ & $\mathrm{ACDE} ; \mathrm{ABCD}$ & $\mathrm{ACE}$ & $\mathrm{ABCD}^{2} ; \mathrm{BD}^{2} \mathrm{E}^{2}$ \\
\hline & & CD & $A B^{2} C^{2} D^{2} E^{2} ; A B^{2} E^{2}$ & $\mathrm{ADE}$ & $\mathrm{ABC}^{2} \mathrm{D} ; \mathrm{BC}^{2} \mathrm{E}^{2}$ \\
\hline & & $C D^{2}$ & $\mathrm{AB}^{2} \mathrm{C}^{2} \mathrm{E}^{2} ; \mathrm{AB}^{2} \mathrm{D}^{2} \mathrm{E}^{2}$ & & \\
\hline & & $\mathrm{CE}$ & $A B^{2} C^{2} D ; A B^{2} D E$ & & \\
\hline & & $\mathrm{CE}^{2}$ & $A B^{2} C^{2} D E ; A B^{2} D$ & & \\
\hline & . & $\mathrm{DE}$ & $A B^{2} C D^{2} ; A B^{2} C E$ & & \\
\hline ' & & $\mathrm{DE}^{2}$ & $\mathrm{AB}^{2} \mathrm{CD}^{2} \mathrm{E} ; \mathrm{AB}^{2} \mathrm{C}$ & . & \\
\hline
\end{tabular}

Vemos pela tabela acima que os efeitos principais e as interações de 2 fatores podem ser estimadas, se as interações de 3 ou mais fatores foram consideradas insignificantes. Assim o esboço do quadro da anālise de variāncia serā: 
C. Variação

G.L.

Efeitos principais

10

Interações de 2 fatores

40

Resíduo

30

Tota1

80

EXEMPLO 2.

Vamos tomar $1 / 9$ do fatorial $3^{6}$. Assim temos a fração $3^{6-2}$. Sejam A, B, C, D, E e $F$ os seis fatores representados por $X_{1}, X_{2}, X_{3}, X_{4}$, $\mathrm{X}_{5}$ e $\mathrm{X}_{6}$ respectivamente, e a relação de identidade escolhida foi $\mathrm{I}=$ $A B C D E^{2}=B C D^{2} F^{2}=A B^{2} C^{2} E^{2} F^{2}=A D^{2} E^{2} F$, onde as duas primeiras interações são escolhidas, e as outras duas são obtidas da seguinte maneira:

$$
\mathrm{ABCDE}^{2} \times \mathrm{BCD}^{2} \mathrm{~F}^{2}=\mathrm{AB}^{2} \mathrm{C}^{2} \mathrm{D}^{3} \mathrm{E}^{2} \mathrm{~F}^{2}=A \mathrm{~B}^{2} \mathrm{C}^{2} \mathrm{E}^{2} \mathrm{~F}^{2}
$$

e

$\mathrm{ABCDE}^{2} \times\left(B C D^{2} \mathrm{~F}^{2}\right)^{2}=\mathrm{ABCDE}^{2} \times \mathrm{B}^{2} \mathrm{C}^{2} \mathrm{DF}=A \mathrm{~B}^{3} \mathrm{C}^{3} \mathrm{D}^{2} \mathrm{E}^{2} \mathrm{~F}=A \mathrm{AD}^{2} \mathrm{E}^{2} \mathrm{~F}$.

Com base nesta relação de identidade acima, temos os seguintes conjuntos de aliases: 


\begin{tabular}{|c|c|c|c|c|c|}
\hline $\begin{array}{l}\text { Efeitos } \\
\text { princi- } \\
\text { pais }\end{array}$ & A1ias & $\begin{array}{c}\text { Interaçōes } \\
\text { de } 2 \\
\text { fatores }\end{array}$ & Alias & $\begin{array}{c}\text { Interaçōes } \\
\text { de } 3 \\
\text { fatores }\end{array}$ & Alias \\
\hline
\end{tabular}

\begin{tabular}{|c|c|c|c|c|c|}
\hline A & $\begin{array}{l}\mathrm{AB}^{2} \mathrm{C}^{2} \mathrm{D}^{2} \mathrm{E} ; \mathrm{ABCD}^{2} \mathrm{~F}^{2} ; \\
\mathrm{ABCEF} ; \mathrm{ADEF} \mathrm{F}^{2} ; \mathrm{BCDE}^{2} ; \\
\mathrm{AB}^{2} \mathrm{C}^{2} \mathrm{DF} ; \mathrm{BCEF} ; \mathrm{DEF}^{2}\end{array}$ & $\mathrm{AB}$ & $\begin{array}{l}\mathrm{ABC}^{2} \mathrm{D}^{2} \mathrm{E} ; \mathrm{AB}^{2} \mathrm{CD}^{2} \mathrm{~F}^{2} ; \\
\mathrm{ACEF} ; \mathrm{AB}^{2} \mathrm{DEF} \mathrm{F}^{2} ; \mathrm{CDE}^{2} ; \\
\mathrm{AC}^{2} \mathrm{DF} ; \mathrm{BC}^{2} \mathrm{E}^{2} \mathrm{~F}^{2} ; \mathrm{BDEF}\end{array}$ & $\mathrm{ABC}^{2}$ & $\begin{array}{l}\mathrm{ABD}^{2} \mathrm{E} ; \mathrm{AB}^{2} \mathrm{D}^{2} \mathrm{~F}^{2} ; \mathrm{AC}^{2} \mathrm{EF} ; \\
\mathrm{AB}^{2} \mathrm{CDEF}^{2} ; \mathrm{CD}^{2} \mathrm{E} ; \\
\mathrm{ACDF} ; \mathrm{BE}^{2} \mathrm{~F}^{2} ; \mathrm{BC}^{2} \mathrm{DEF}{ }^{2}\end{array}$ \\
\hline B & $\begin{array}{l}\mathrm{AB}^{2} \mathrm{CDE}^{2} ; \mathrm{BC}^{2} \mathrm{DF} ; \mathrm{AC}^{2} \mathrm{E}^{2} \mathrm{~F}^{2} ; \\
\mathrm{ABD}^{2} \mathrm{E}^{2} \mathrm{~F} ; \mathrm{ACDE}^{2} ; \mathrm{CD}^{2} \mathrm{~F}^{2} ; \\
\mathrm{ABC}^{2} \mathrm{E}^{2} \mathrm{~F}^{2} ; \mathrm{AB}^{2} \mathrm{D}^{2} \mathrm{E}^{2} \mathrm{~F}\end{array}$ & $\mathrm{AB}^{2}$ & $\begin{array}{l}\mathrm{AC}^{2} \mathrm{D}^{2} \mathrm{E} ; \mathrm{ACD}^{2} \mathrm{~F}^{2} ; \mathrm{AB}^{2} \mathrm{CEF} ; \\
\mathrm{ABDEF}^{2} ; \mathrm{BC}^{2} \mathrm{D}^{2} \mathrm{E} ; \\
\mathrm{ABC}^{2} \mathrm{DF} ; \mathrm{CEF} ; \mathrm{BDEF}^{2}\end{array}$ & $\mathrm{AB}^{2} \mathrm{C}$ & $\begin{array}{l}\mathrm{ACD}^{2} \mathrm{E}^{2} ; \mathrm{AC}^{2} \mathrm{D}^{2} \mathrm{~F}^{2} ; \mathrm{AB}^{2} \mathrm{EF} ; \\
\mathrm{ABC}^{2} \mathrm{DEF} \mathrm{F}^{2} ; \mathrm{BD}^{2} \mathrm{E} ; \mathrm{ABDF} ; \\
\mathrm{CE}^{2} \mathrm{~F}^{2} ; \mathrm{BC}^{2} \mathrm{D}^{2} \mathrm{E}^{2} \mathrm{~F}\end{array}$ \\
\hline C & $\begin{array}{l}\mathrm{ABC}^{2} \mathrm{DE}^{2} ; \mathrm{BC}^{2} \mathrm{D}^{2} \mathrm{~F}^{2} ; \\
\mathrm{AB}^{2} \mathrm{E}^{2} \mathrm{~F}^{2} ; \mathrm{ACD}^{2} \mathrm{E}^{2} \mathrm{~F} ; \mathrm{ABDE}^{2} ; \\
\mathrm{BD}^{2} \mathrm{~F}^{2} ; \mathrm{AB}^{2} \mathrm{CE}^{2} \mathrm{~F}^{2} ; \mathrm{AC}^{2} \mathrm{D}^{2} \mathrm{E}^{2} \mathrm{~F}\end{array}$ & $\mathrm{AC}$ & $\begin{array}{l}\mathrm{AB}^{2} \mathrm{CD}^{2} \mathrm{E} ; \mathrm{ABC}^{2} \mathrm{D}^{2} \mathrm{~F}^{2} ; \\
\mathrm{ABEF} ; \mathrm{AC}^{2} \mathrm{DEF}^{2} ; \mathrm{BDE}^{2} ; \\
\mathrm{AB}^{2} \mathrm{DF} ; \mathrm{BC}^{2} \mathrm{EF} ; \mathrm{CDEF}\end{array}$ & $\mathrm{ABD}^{2}$ & $\begin{array}{l}\mathrm{ABC}^{2} \mathrm{E} ; \mathrm{AB}^{2} \mathrm{CDF}^{2} ; \mathrm{ACDEF} ; \\
\mathrm{AB}^{2} \mathrm{D}^{2} \mathrm{EF}^{2} ; \mathrm{CD}^{2} \mathrm{E}^{2} ; \mathrm{AC}^{2} \mathrm{~F} ; \\
\mathrm{BC}^{2} \mathrm{DE}^{2} \mathrm{~F}^{2} ; \mathrm{BEF}^{2}\end{array}$ \\
\hline D & $\begin{array}{l}\mathrm{ABCD}^{2} \mathrm{E}^{2} ; \mathrm{BCF}^{2} ; \mathrm{AB}^{2} \mathrm{C}^{2} \mathrm{DE}^{2} \mathrm{~F}^{2} ; \\
\mathrm{AE}^{2} \mathrm{~F} ; \mathrm{ABCE}^{2} ; \mathrm{BCDF}^{2} ; \\
\mathrm{AB}^{2} \mathrm{C}^{2} \mathrm{D}^{2} \mathrm{E}^{2} \mathrm{~F}^{2} ; \mathrm{ADE}^{2} \mathrm{~F}\end{array}$ & $\mathrm{AC}^{2}$ & $\begin{array}{l}\mathrm{AB}^{2} \mathrm{D}^{2} \mathrm{E} ; \mathrm{ABD}^{2} \mathrm{~F}^{2} ; \mathrm{ABC}^{2} \mathrm{EF} ; \\
\mathrm{ACEF^{2 }} ; \mathrm{BC}^{2} \mathrm{DE}^{2} ; \mathrm{AB}^{2} \mathrm{CDF} ; \\
\mathrm{BEF} ; \mathrm{CD}^{2} \mathrm{E}^{2} \mathrm{~F}\end{array}$ & $A B^{2} D$ & $\begin{array}{l}\mathrm{AC}^{2} \mathrm{DE} ; \mathrm{ACF}^{2} ; \mathrm{AB}^{2} \mathrm{CD}^{2} \mathrm{EF} ; \\
\mathrm{ABEF}^{2} ; \mathrm{BC}^{2} \mathrm{E} ; \mathrm{ABC}^{2} \mathrm{D}^{2} \mathrm{~F} ; \\
\mathrm{BCDEF} ; \mathrm{BDE}^{2} \mathrm{~F}\end{array}$ \\
\hline$E$ & $\begin{array}{l}\mathrm{ABCD} ; \mathrm{BCD}^{2} \mathrm{EF}{ }^{2} ; \mathrm{AB}^{2} \mathrm{C}^{2} \mathrm{~F}^{2} ; \\
\mathrm{AD}^{2} \mathrm{~F} ; \mathrm{ABCDE} ; \mathrm{BCD}^{2} \mathrm{E}^{2} \mathrm{~F}^{2} ; \\
\mathrm{AB}^{2} \mathrm{C}^{2} \mathrm{EF} F^{2} ; \mathrm{AD}^{2} \mathrm{GF}\end{array}$ & $\mathrm{AD}$ & $\begin{array}{l}\mathrm{AB}^{2} \mathrm{C}^{2} \mathrm{DE} ; \mathrm{ABCF}^{2} \\
\mathrm{ABCD}^{2} \mathrm{EF} ; \mathrm{AEF}^{2} ; \mathrm{BCE}^{2} ; \\
\mathrm{AB}^{2} \mathrm{C}^{2} \mathrm{D}^{2} \mathrm{~F} ; \mathrm{BCD}^{2} \mathrm{EF} ; \mathrm{DE}^{2} \mathrm{~F} ;\end{array}$ & $A B^{2} D^{2}$ & $\begin{array}{l}\mathrm{AC}^{2} \mathrm{E} ; \mathrm{ACDF}^{2} ; \mathrm{AB}^{2} \mathrm{CDEF} ; \\
\mathrm{ABD}^{2} \mathrm{EF}^{2} ; \mathrm{BC}^{2} \mathrm{DE} ; \mathrm{ABC}^{2} \mathrm{~F} ; \\
\mathrm{CD}^{2} \mathrm{EF} ; \mathrm{BE}^{2} \mathrm{~F}\end{array}$ \\
\hline $\mathrm{F}$ & $\begin{array}{l}\mathrm{ABCDE}{ }^{2} \mathrm{~F} ; \mathrm{BCD}^{2} ; \mathrm{AB}^{2} \mathrm{C}^{2} \mathrm{E}^{2} ; \\
\mathrm{AD}^{2} \mathrm{E}^{2} \mathrm{~F}^{2} ; \mathrm{ABCDE}^{2} \mathrm{~F}^{2} ; \\
\mathrm{BCD}^{2} \mathrm{~F} ; \mathrm{AB}^{2} \mathrm{C}^{2} \mathrm{E}^{2} \mathrm{~F} ; \mathrm{AD}^{2} \mathrm{E}^{2}\end{array}$ & $A D^{2}$ & $\begin{array}{l}\mathrm{AB}^{2} \mathrm{C}^{2} \mathrm{E} ; \mathrm{ABCDF}^{2} ; \\
\mathrm{ABCDEF} ; \mathrm{AD}^{2} \mathrm{EF}^{2} ; \mathrm{BCD}^{2} \mathrm{E}^{2} ; \\
\mathrm{AB}^{2} \mathrm{C}^{2} \mathrm{~F} ; \mathrm{BCD}^{2} \mathrm{EF} ; \mathrm{EF}^{2}\end{array}$ & $\mathrm{ABE}$ & $\begin{array}{l}\mathrm{ABC}^{2} \mathrm{D}^{2} ; \mathrm{AB}^{2} \mathrm{CD}^{2} \mathrm{EF}^{2} ; \\
\mathrm{ACF}, \mathrm{AB}^{2} \mathrm{DF}^{2} ; \mathrm{CDE}, \mathrm{AC}^{2} \mathrm{DEF} ; \\
\mathrm{BC}^{2} \mathrm{EF}^{2} ; \mathrm{BDE}^{2} \mathrm{~F}^{2}\end{array}$ \\
\hline & & $\mathrm{AE}$ & $\begin{array}{l}\mathrm{AB}^{2} \mathrm{C}^{2} \mathrm{D}^{2} ; \mathrm{ABCD} \mathrm{ABF}^{2} \mathrm{EF}^{2} ; \\
\mathrm{ABCF} ; \mathrm{ADF} \mathrm{F}^{2} ; \mathrm{BCDE} ; \\
\mathrm{AB}^{2} \mathrm{C}^{2} \mathrm{DEF} ; \mathrm{BCE}^{2} \mathrm{~F} ; \mathrm{DE}^{2} \mathrm{~F}^{2}\end{array}$ & $A B^{2} E$ & $\begin{array}{l}\mathrm{AC}^{2} \mathrm{D}^{2} ; \mathrm{ACD}^{2} \mathrm{EF}^{2} ; \mathrm{AB}^{2} \mathrm{CF} ; \\
\mathrm{ABDF}^{2} ; \mathrm{BC}^{2} \mathrm{D}^{2} \mathrm{E}^{2} ; \\
\mathrm{ABC}^{2} \mathrm{DEF} ; \mathrm{CE}^{2} \mathrm{~F} ; \mathrm{BD}^{2} \mathrm{EF}\end{array}$ \\
\hline & & $\mathrm{AE}^{2}$ & $\begin{array}{l}\mathrm{AB}^{2} \mathrm{C}^{2} \mathrm{D}^{2} \mathrm{E}^{2} ; \mathrm{ABCD}^{2} \mathrm{E}^{2} \mathrm{~F}^{2} ; \\
\mathrm{ABCE^{2 } \mathrm { F } ; \mathrm { ADE }} \mathrm{AD}^{2} \mathrm{~F} ; \mathrm{BCD} ; \\
\mathrm{AB}^{2} \mathrm{C}^{2} \mathrm{DE} \mathrm{E}^{2} \mathrm{~F} ; \mathrm{BCF} ; \mathrm{DF}^{2}\end{array}$ & $\mathrm{ABF}$ & $\begin{array}{l}\mathrm{ABC}^{2} \mathrm{D}^{2} \mathrm{EF}^{2} ; \mathrm{AB}^{2} \mathrm{CD}^{2} ; \mathrm{ACE} ; \\
\mathrm{AB}^{2} \mathrm{DEF} ; \mathrm{CDE}^{2} \mathrm{~F}^{2} ; \mathrm{AC}^{2} \mathrm{DF} ; \\
\mathrm{BC}^{2} \mathrm{E}^{2} \mathrm{~F} ; \mathrm{BDE}\end{array}$ \\
\hline & & $\mathrm{AF}$ & $\begin{array}{l}\mathrm{AB}^{2} \mathrm{C}^{2} \mathrm{D}^{2} \mathrm{EF}^{2} ; \mathrm{ABCD}^{2} ; \\
\mathrm{ABCE} ; \mathrm{ADEF} ; \mathrm{BCDE}^{2} \mathrm{~F}^{2} ; \\
\mathrm{AC}^{2} \mathrm{DF} ; \mathrm{BCEF}^{2} ; \mathrm{DE}\end{array}$ & $\mathrm{ABF}^{2}$ & $\begin{array}{l}\mathrm{ABC}^{2} \mathrm{D}^{2} \mathrm{EF} ; \mathrm{AB}^{2} \mathrm{CD}^{2} \mathrm{~F} ; \\
\mathrm{ACEF}^{2} ; \mathrm{AB}^{2} \mathrm{DE} ; \mathrm{CDE}^{2} \mathrm{~F} ; \mathrm{AC}^{2} \mathrm{D} ; \\
\mathrm{BC}^{2} \mathrm{E}^{2} ; \mathrm{BDEF}\end{array}$ \\
\hline & . & $\mathrm{AF}^{2}$ & $\begin{array}{l}\mathrm{AB}^{2} \mathrm{C}^{2} \mathrm{D}^{2} \mathrm{EF} ; \mathrm{ABCD}^{2} \mathrm{~F} ; \\
\mathrm{ABCEF}^{2} ; \mathrm{ADE} ; \mathrm{BCDE}^{2} \mathrm{~F} ; \\
\mathrm{AB}^{2} \mathrm{C}^{2} \mathrm{D} ; \mathrm{BCE} ; \mathrm{DEF}\end{array}$ & $\mathrm{AB}^{2} \mathrm{~F}$ & $\begin{array}{l}\mathrm{AC}^{2} \mathrm{D}^{2} \mathrm{EF}^{2} ; \mathrm{ACD}^{2} ; \mathrm{AB}^{2} \mathrm{CE} ; \\
\mathrm{ABDEF} ; \mathrm{BC}^{2} \mathrm{D}^{2} \mathrm{EF} ; \\
\mathrm{ABC}^{2} \mathrm{DF}^{2} ; \mathrm{CEF}^{2} ; \mathrm{BD}^{2} \mathrm{E}^{2}\end{array}$ \\
\hline & & $\mathrm{BC}$ & $\begin{array}{l}\mathrm{AB}^{2} \mathrm{C}^{2} \mathrm{DE}^{2} ; \mathrm{BCDF} ; \mathrm{AE}^{2} \mathrm{~F}^{2} \\
\mathrm{ABCD}^{2} \mathrm{E}^{2} \mathrm{~F} ; \mathrm{ADE}^{2} ; \mathrm{DF} ; \\
\mathrm{ABCD}^{2} \mathrm{~F}^{2} ; \mathrm{AB}^{2} \mathrm{C}^{2} \mathrm{D}^{2} \mathrm{E}^{2} \mathrm{~F}\end{array}$ & & \\
\hline
\end{tabular}




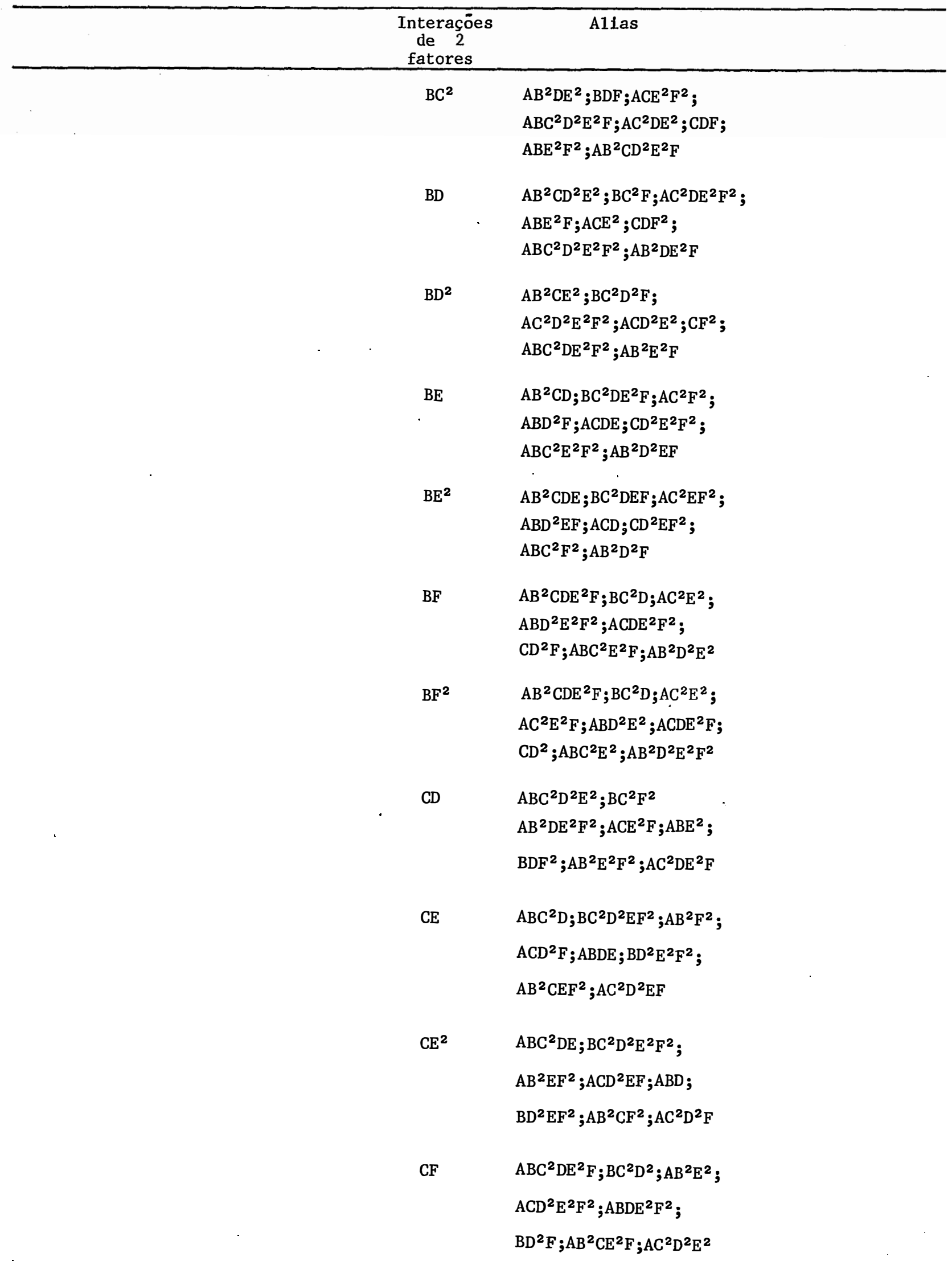




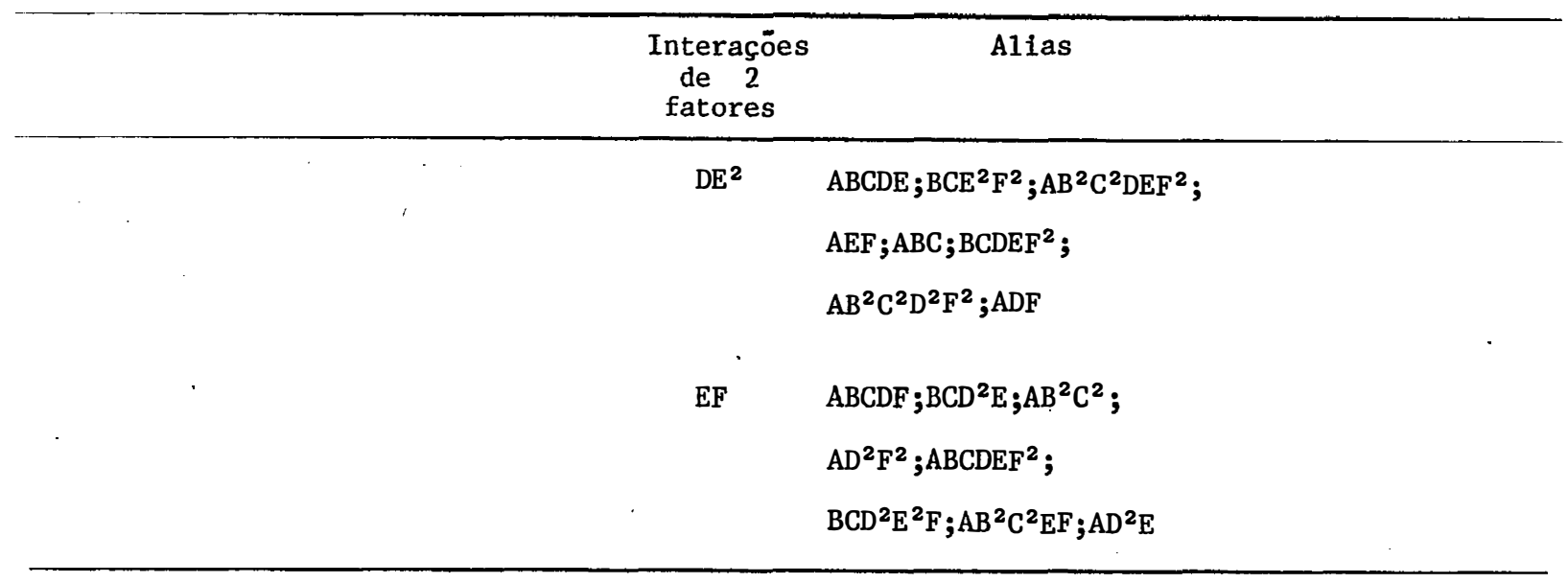

Como vemos pelos conjuntos de aliases acima, o experimen to contendo $1 / 9$ do fatorial $3^{6}$, confunde algumas interações de fatores com outras, também de 2 fatores, como por exemplo, $\mathrm{AD}^{2} \mathrm{com} \mathrm{EF}^{2}, \mathrm{AE}^{2} \mathrm{com}$ $\mathrm{DF}^{2}, \mathrm{AF}$ com $\mathrm{DE}, \mathrm{BC}$ com $\mathrm{DF}, \mathrm{BD}^{2}$ com $\mathrm{CF}^{2}$ e $\mathrm{BF}^{2}$ com $\mathrm{CD}^{2}$.

Segundo LIMA (1985), para $\mathrm{n}=6$, o fatorial $3^{5}$ è o nümero mínimo de unidades que suporta a resolução $\mathrm{V}$, i.è, aqueles experimen tos constituidos de frações nas quais todos os efeitos principais e interações de 2 fatores são estimäveis. Para os fatoriais $3^{n}, \operatorname{com} n \geqq 7$, temos conjuntos de efeitos e interações com números muito grande de ele mentos. Para obtenção da fração $3^{7-1}$, o conjunto de efeitos e interações constitui-se de 364 elementos. A isso se deve o pouco uso dos fato riais fracionados dessa sërie.

No nosso exemplo, a relação de identidade escolhida foi $\mathrm{I}=\mathrm{ABCDE} \mathrm{E}^{2}=\mathrm{BCD}^{2} \mathrm{~F}^{2}=\mathrm{AB}^{2} \mathrm{C}^{2} \mathrm{E}^{2} \mathrm{~F}^{2}=\mathrm{AD}^{2} \mathrm{E}^{2} \mathrm{~F}$. Tomando como base esta relação, as nove frações são constituidas da seguinte maneira: 
FRAÇđ̃o I: Serā constituida das combinações de tratamentos que satisfazem as seguintes equações:

$$
\begin{aligned}
& \mathrm{X}_{1}+\mathrm{x}_{2}+\mathrm{x}_{3}+\mathrm{x}_{4}+2 \mathrm{x}_{5}=0(\bmod .3) \text { ou } \mathrm{ABCDE}_{\mathrm{O}}^{2} \\
& \mathrm{x}_{2}+\mathrm{X}_{3}+2 \mathrm{x}_{4}+2 \mathrm{x}_{6}=0(\bmod .3) \text {, ou } \mathrm{BCD}^{2} \mathrm{~F}_{\mathrm{O}}^{2} \\
& \mathrm{X}_{1}+2 \mathrm{X}_{2}+2 \mathrm{X}_{3}+2 \mathrm{X}_{5}+2 \mathrm{x}_{6}=0(\bmod 3) \text {, ou }\left(A B^{2} \mathrm{C}^{2} \mathrm{E}^{2} \mathrm{~F}^{2}\right)_{\mathrm{o}} \\
& \mathrm{X}_{1}+2 \mathrm{x}_{4}+2 \mathrm{x}_{5}+\mathrm{X}_{6}=0(\bmod 3) \text {, ou }\left(\mathrm{AD}^{2} \mathrm{E}^{2} \mathrm{~F}\right)_{0}
\end{aligned}
$$

onde, as duas ültimas equações são obtidas atravēs de operações sobre as duas primeiras da seguinte maneira:

$$
\begin{aligned}
& \mathrm{x}_{1}+\mathrm{x}_{2}+\mathrm{x}_{3}+\mathrm{x}_{4}+2 \mathrm{x}_{5}=0 \text { (mod. 3) } \\
& \mathrm{x}_{2}+\mathrm{x}_{3}+2 \mathrm{x}_{4}+\quad+2 \mathrm{x}_{6}=0(\bmod .3) \\
& x_{1}+2 x_{2}+2 x_{3}+3 x_{4}+2 x_{5}+2 x_{6}=0(\bmod .3) \Longrightarrow x_{1}+2 x_{2}+2 x_{3}+2 x_{5}+2 x_{6}=0 \text { (mod. 3) } \\
& \mathrm{x}_{1}+\mathrm{x}_{2}+\mathrm{x}_{3}+\mathrm{x}_{4}+2 \mathrm{x}_{5}=0 \text { (mod. 3) } \\
& 2\left(\mathrm{x}_{2}+\mathrm{x}_{3}+2 \mathrm{x}_{4}++2 \mathrm{x}_{6}\right)=2 \times 0(\bmod .3) \\
& x_{1}+3 x_{2}+3 x_{3}+5 x_{4}+2 x_{5}+4 x_{6}=0 \quad(\bmod .3) \Rightarrow x_{1}+2 x_{4}+2 x_{5}+x_{6}=0(\bmod .3)
\end{aligned}
$$

FRAÇÃo II: Serā constituida das combinações de tratamentos que satisfazem as equações:

$$
\begin{aligned}
& \mathrm{x}_{1}+\mathrm{x}_{2}+\mathrm{x}_{3}+\mathrm{x}_{4}+2 \mathrm{x}_{5}=0 \quad(\bmod .3), \quad \text { ou } \mathrm{ABCDE}_{\mathrm{O}}^{2} \\
& \mathrm{x}_{2}+\mathrm{x}_{3}+2 \mathrm{x}_{4}+2 \mathrm{x}_{6}=1(\bmod , 3), \quad \text { ou } \mathrm{BCD}^{2} \mathrm{~F}_{1}^{2}
\end{aligned}
$$

e as outras duas equações são: 


$$
\begin{aligned}
& \mathrm{x}_{1}+\mathrm{x}_{2}+\mathrm{x}_{3}+\mathrm{x}_{4}+2 \mathrm{x}_{5}=0(\bmod .3) \\
& \mathrm{x}_{2}+\mathrm{x}_{3}+2 \mathrm{x}_{4}++2 \mathrm{x}_{6}=1(\bmod .3) \\
& \mathrm{x}_{1}+2 \mathrm{x}_{2}+2 \mathrm{x}_{3}+3 \mathrm{x}_{4}+2 \mathrm{x}_{5}+2 \mathrm{x}_{6}=1 \stackrel{\mathrm{g}}{\Rightarrow} \mathrm{x}_{1}+2 \mathrm{x}_{2}+2 \mathrm{x}_{3}+2 \mathrm{x}_{5}+2 \mathrm{x}_{6}=1 \text { (mod. 3), ou } \\
& \mathrm{AB}^{2} \mathrm{C}^{2} \mathrm{E}^{2} \mathrm{~F}_{1}^{2} \\
& \mathrm{x}_{1}+\mathrm{x}_{2}+\mathrm{x}_{3}+\mathrm{x}_{4}+2 \mathrm{x}_{5}=0 \quad(\bmod .3) \\
& 2\left(\mathrm{x}_{2}+\mathrm{x}_{3}+2 \mathrm{x}_{4}++2 \mathrm{x}_{6}\right)=2(\bmod \cdot 3) \\
& x_{1}+3 x_{2}+3 x_{3}+5 x_{4}+2 x_{5}+4 x_{6}=2 \Rightarrow x_{1}+2 x_{4}+2 x_{5}+x_{6}=2 \text { (mod. 3), ou } \\
& \mathrm{AD}^{2} \mathrm{E}^{2} \mathrm{~F}_{2}
\end{aligned}
$$

FRAÇÃo III: Satisfaz as equações obtidas como nas frações anteriores:

$$
\begin{array}{llll}
\mathrm{X}_{1}+\mathrm{X}_{2}+\mathrm{X}_{3}+\mathrm{X}_{4}+2 \mathrm{X}_{5}=0 & (\bmod .3), & \text { ou } & \mathrm{ABCDE}_{\mathrm{O}}^{2} \\
\mathrm{X}_{2}+\mathrm{X}_{3}+2 \mathrm{X}_{4}+2 \mathrm{X}_{6}=2 & (\bmod .3), & \text { ou } & \mathrm{BCD}^{2} \mathrm{~F}_{2}^{2} \\
\mathrm{X}_{1}+2 \mathrm{x}_{2}+2 \mathrm{X}_{3}+2 \mathrm{X}_{5}+2 \mathrm{X}_{6}=2 & (\bmod .3), & \text { ou } & \mathrm{AB}^{2} \mathrm{C}^{2} \mathrm{E}^{2} \mathrm{~F}_{2}^{2} \\
\mathrm{X}_{1}+2 \mathrm{x}_{4}+2 \mathrm{X}_{5}+\mathrm{X}_{6}=1 & (\bmod .3), & \text { ou } & \mathrm{AD}^{2} \mathrm{E}^{2} \mathrm{~F}_{1}
\end{array}
$$

\section{FRAĢÃo IV:}

$$
\begin{aligned}
& \mathrm{x}_{1}+\mathrm{x}_{2}+\mathrm{x}_{3}+\mathrm{x}_{4}+2 \mathrm{x}_{5}=1 \quad(\bmod .3) \text {, ou } \mathrm{ABCDE}_{1}^{2} \\
& \mathrm{x}_{2}+\mathrm{x}_{3}+2 \mathrm{x}_{4}+2 \mathrm{x}_{6}=0 \quad(\bmod .3) \text {, ou } \mathrm{BCD}^{2} \mathrm{~F}_{\mathrm{o}}^{2} \\
& \mathrm{X}_{1}+2 \mathrm{x}_{2}+2 \mathrm{X}_{3}+2 \mathrm{X}_{5}+2 \mathrm{X}_{6}=1(\bmod .3) \text {, ou } \mathrm{AB}^{2} \mathrm{C}^{2} \mathrm{E}^{2} \mathrm{~F}_{1}^{2} \\
& \mathrm{X}_{1}+2 \mathrm{X}_{4}+2 \mathrm{X}_{5}+\mathrm{X}_{6}=1 \quad(\bmod .3) \text {, ou } \quad \mathrm{AD}^{2} \mathrm{E}^{2} \cdot \mathrm{F}_{1}
\end{aligned}
$$




\section{FRAÇÃO V:}

$$
\begin{aligned}
& \mathrm{x}_{1}+\mathrm{x}_{2}+\mathrm{x}_{3}+\mathrm{x}_{4}+2 \mathrm{x}_{5}=1 \quad(\bmod .3) \text {, ou } \mathrm{ABCDE}_{1}^{2} \\
& \mathrm{x}_{2}+\mathrm{x}_{3}+2 \mathrm{x}_{4}+2 \mathrm{x}_{6}=1 \quad(\bmod .3) \text {, ou } \mathrm{BCD}^{2} \mathrm{~F}_{1}^{2} \\
& \mathrm{x}_{1}+2 \mathrm{x}_{2}+2 \mathrm{x}_{3}+2 \mathrm{x}_{5}+2 \mathrm{x}_{6}=2(\bmod .3) \text {, ou } \mathrm{AB}^{2} \mathrm{C}^{2} \mathrm{E}^{2} \mathrm{~F}_{2}^{2} \\
& \mathrm{X}_{1}+2 \mathrm{x}_{4}+2 \mathrm{x}_{5}+\mathrm{X}_{6}=0 \quad(\bmod .3) \text {, ou } \mathrm{AD}^{2} \mathrm{E}^{2} \mathrm{~F}_{0}
\end{aligned}
$$

\section{FRAÇÃO VI:}

$$
\begin{aligned}
& \mathrm{x}_{1}+\mathrm{x}_{2}+\mathrm{x}_{3}+\mathrm{x}_{4}+2 \mathrm{x}_{5}=1 \quad(\bmod \cdot 3) \text {, ou } \mathrm{ABCDE}_{1}^{2} \\
& \mathrm{x}_{2}+\mathrm{x}_{3}+2 \mathrm{x}_{4}+2 \mathrm{x}_{6}=2 \quad(\bmod .3) \text {, ou } \mathrm{BCD}^{2} \mathrm{~F}_{2}^{2} \\
& \mathrm{x}_{1}+2 \mathrm{x}_{2}+2 \mathrm{x}_{3}+2 \mathrm{x}_{5}+2 \mathrm{x}_{6}=0 \quad(\bmod .3) \text {, ou } A B^{2} \mathrm{C}^{2} \mathrm{E}^{2} \mathrm{~F}_{0}^{2} \\
& \mathrm{X}_{1}+2 \mathrm{x}_{4}+2 \mathrm{x}_{5}+\mathrm{X}_{6}=2 \quad(\bmod .3), \text { ou } \mathrm{AD}^{2} \mathrm{E}^{2} \mathrm{~F}_{2}
\end{aligned}
$$

\section{FRAÇÃO : VI I}

$$
\begin{aligned}
& \mathrm{x}_{1}+\mathrm{x}_{2}+\mathrm{x}_{3}+\mathrm{x}_{4}+2 \mathrm{x}_{5}=2 \quad(\bmod .3) \text {, ou } \mathrm{ABCDE}_{2}^{2} \\
& \mathrm{x}_{2}+\mathrm{X}_{3}+2 \mathrm{x}_{4}+2 \mathrm{x}_{6}=0 \quad(\bmod .3), \text { ou } \mathrm{BCD}^{2} \mathrm{~F}_{0}^{2} \\
& \mathrm{x}_{1}+2 \mathrm{x}_{2}+2 \mathrm{x}_{3}+2 \mathrm{x}_{5}+2 \mathrm{x}_{6}=2(\bmod , 3) \text {, ou } \mathrm{AB}^{2} \mathrm{C}^{2} \mathrm{E}^{2} \mathrm{~F}_{2}^{2} \\
& \mathrm{X}_{1}+2 \mathrm{x}_{4}+2 \mathrm{x}_{5}+\mathrm{X}_{6}=2 \quad(\bmod .3), \text { ou } \mathrm{AD}^{2} \mathrm{E}^{2} \mathrm{~F}_{2}
\end{aligned}
$$




\section{FRAÇÃO VIIII}

$$
\begin{aligned}
& \mathrm{x}_{1}+\mathrm{x}_{2}+\mathrm{x}_{3}+\mathrm{x}_{4}+2 \mathrm{x}_{5}=2 \quad(\bmod .3) \text {, ou } \mathrm{ABCDE}_{2}^{2} \\
& \mathrm{X}_{2}+\mathrm{X}_{3}+2 \mathrm{x}_{4}+2 \mathrm{x}_{6}=1 \quad(\bmod .3) \text {, ou } \mathrm{BCD}^{2} \mathrm{~F}_{1}^{2} \\
& \mathrm{X}_{1}+2 \mathrm{X}_{2}+2 \mathrm{x}_{3}+2 \mathrm{X}_{5}+2 \mathrm{x}_{6}=0 \quad(\bmod .3) \text {, ou } \mathrm{AB}^{2} \mathrm{C}^{2} \mathrm{E}^{2} \mathrm{~F}_{\mathrm{o}}^{2} \\
& \mathrm{X}_{1}+2 \mathrm{X}_{4}+2 \mathrm{X}_{5}+\mathrm{X}_{6}=1 \quad(\bmod .3) \text {, ou } \mathrm{AD}^{2} \mathrm{E}^{2} \mathrm{~F}_{11}
\end{aligned}
$$

\section{FRAÇÃo IX:}

$$
\begin{aligned}
& \mathrm{x}_{1}+\mathrm{x}_{2}+\mathrm{x}_{3}+\mathrm{x}_{4}+2 \mathrm{x}_{5}=2 \quad(\bmod .3), \quad \text { ou } \mathrm{ABCDE}_{2}^{2} \\
& \mathrm{X}_{2}+\mathrm{X}_{3}+2 \mathrm{x}_{4}+2 \mathrm{x}_{6}=2 \quad(\bmod .3) \text {, ou } \mathrm{BCD}^{2} \mathrm{~F}_{2}^{2} \\
& \mathrm{X}_{1}+2 \mathrm{X}_{2}+2 \mathrm{X}_{3}+2 \mathrm{X}_{5}+2 \mathrm{X}_{6}=1 \quad(\bmod .3) \text {, ou } \mathrm{AB}^{2} \mathrm{C}^{2} \mathrm{E}^{2} \mathrm{~F}_{1}^{2} \text {, } \\
& \mathrm{X}_{1}+2 \mathrm{x}_{4}+2 \mathrm{x}_{5}+\mathrm{x}_{6}=0 \quad(\bmod 3) \text {, ou } \mathrm{AD}^{2} \mathrm{E}^{2} \mathrm{~F}_{0}
\end{aligned}
$$

Como vimos do quadro das aliases temos 6 pares de intera ções de 2 fatores confundind $s$. Se fosse considerada uma das duas inte rações como insignificantes, teriamos o seguinte esboço da anālise de variância. 


\begin{tabular}{ll}
\hline C. Variação & G.L. \\
\hline Efeitos principais & 12 \\
Interações de 2 fatores & 48 (6 interações confundidas) \\
Residuo & 20 \\
\hline Total & 80 \\
\hline
\end{tabular}

\subsection{Fatoriais fracionados da série $3^{n}$ em blocos ao acáso}

No caso dos fatoriais fracionados da série $3^{\text {n }}$, como vamos trabalhar necessariamente com $\mathrm{n} \geqq 5$, os experimentos são compostos por um nümero batante elevado de combinações de tratamentos, o que nos leva a lançar mão de delineamentos em blocos ao acaso com confundimento. Para exemplificar vamos tomar $1 / 3$ do fatorial $3^{5}$.

EXXMPLO: Seja a fração $3^{5-1}$, sendo colocada em 3 blocos de $3^{3}$ combinações de tratamentos. Para isto, precisamos confundir apenas uma intera ção com blocos, e vamos escolher o experimento dado pela relação de Identidade $I=\mathrm{AB}^{2} \mathrm{CDE}_{0}^{2}$, ou seja, aquele em que a soma dos números modulares das combinações de tratamentos è igual a 0 (mod. 3).

A interação escolhida para confundir com blocos è $\mathrm{BC}^{2} \mathrm{D}$, pois as suas generalizadas com aquelas interações da relação de identidade, são interações de 3 ou ma is fatores, conforme pode ser visto no 
.106.

quadro de allases, isto é,

$$
B C^{2} \mathrm{D}=A \mathrm{AD}^{2} \mathrm{E}^{2}=A B C^{2} \mathrm{E}^{2} .
$$

0s 3 blocos são os seguintes: 
Bloco I

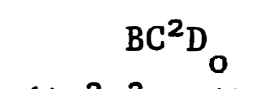

Bloco II

$B C^{2} D_{1}$
Bloco III

$\mathrm{BC}^{2} \mathrm{D}_{2}$

$\left(\mathrm{AD}^{2} \mathrm{E}_{1}^{2} ; \mathrm{ABC}^{2} \mathrm{E}_{2}^{2}\right)$

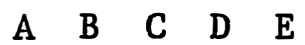

$\begin{array}{lllll}1 & 1 & 0 & 0 & 0\end{array}$

$\begin{array}{lllll}1 & 2 & 1 & 0 & 0\end{array}$

$1 \begin{array}{llll}0 & 2 & 0 & 0\end{array}$

$\begin{array}{lllll}2 & 0 & 0 & 1 & 0\end{array}$

$\begin{array}{lllll}2 & 1 & 1 & 1 & 0\end{array}$

$\begin{array}{lllll}2 & 2 & 2 & 1 & 0\end{array}$

$\begin{array}{lllll}0 & 2 & 0 & 2 & 0\end{array}$

$\begin{array}{lllll}0 & 0 & 1 & 2 & 0\end{array}$

$\begin{array}{lllll}0 & 1 & 2 & 2 & 0\end{array}$

$\begin{array}{lllll}2 & 1 & 0 & 0 & 1\end{array}$

$\begin{array}{lllll}2 & 2 & 1 & 0 & 1\end{array}$

$\begin{array}{lllll}2 & 0 & 2 & 0 & 1\end{array}$

$\begin{array}{lllll}0 & 0 & 0 & 1 & 1\end{array}$

$\begin{array}{lllll}0 & 1 & 1 & 1 & 1\end{array}$

$\begin{array}{lllll}0 & 2 & 2 & 1 & 1\end{array}$

$\begin{array}{lllll}1 & 2 & 0 & 2 & 1\end{array}$

$\begin{array}{lllll}1 & 0 & 1 & 2 & 1\end{array}$

$\begin{array}{lllll}1 & 1 & 2 & 2 & 1\end{array}$

$\begin{array}{lllll}0 & 1 & 0 & 0 & 2\end{array}$

$\begin{array}{lllll}0 & 2 & 1 & 0 & 2\end{array}$;

$\begin{array}{lllll}0 & 0 & 2 & 0 & 2\end{array}$

$\begin{array}{lllll}1 & 0 & 0 & 1 & 2\end{array}$

$\begin{array}{lllll}1 & 1 & 1 & 1 & 2\end{array}$

$\begin{array}{lllll}1 & 2 & 2 & 1 & 2\end{array}$

$\begin{array}{lllll}2 & 2 & 0 & 2 & 2\end{array}$

$\begin{array}{lllll}2 & 0 & 1 & 2 & 2\end{array}$

$\begin{array}{lllll}2 & 1 & 2 & 2 & 2\end{array}$
$\left(A D^{2} E_{2}^{2} ; A B C^{2} E_{1}^{2}\right)$

A $B \quad C \quad D \quad E$

$\begin{array}{lllll}2 & 2 & 0 & 0 & 0\end{array}$

$\begin{array}{lllll}2 & 0 & 1 & 0 & 0\end{array}$

$\begin{array}{lllll}2 & 1 & 2 & 0 & 0\end{array}$

$\begin{array}{lllll}0 & 1 & 0 & 1 & 0\end{array}$

$\begin{array}{lllll}0 & 2 & 1 & 1 & 0\end{array}$

$\begin{array}{lllll}0 & 0 & 2 & 1 & 0\end{array}$

$\begin{array}{lllll}1 & 0 & 0 & 2 & 0\end{array}$

$\begin{array}{lllll}1 & 1 & 1 & 2 & 0\end{array}$

$\begin{array}{lllll}1 & 2 & 2 & 2 & 0\end{array}$

$\begin{array}{lllll}0 & 2 & 0 & 0 & 1\end{array}$

$\begin{array}{lllll}0 & 0 & 1 & 0 & 1\end{array}$

$\begin{array}{lllll}0 & 1 & 2 & 0 & 1\end{array}$

$\begin{array}{lllll}1 & 1 & 0 & 1 & 1\end{array}$

$\begin{array}{lllll}1 & 2 & 1 & 1 & 1\end{array}$

$\begin{array}{lllll}1 & 0 & 2 & 1 & 1\end{array}$

$\begin{array}{lllll}2 & 0 & 0 & 2 & 1\end{array}$

$\begin{array}{lllll}2 & 1 & 1 & 2 & 1\end{array}$

$\begin{array}{lllll}2 & 2 & 2 & 2 & 1\end{array}$

$\begin{array}{lllll}1 & 2 & 0 & 0 & 2\end{array}$

$\begin{array}{lllll}1 & 0 & 1 & 0 & 2\end{array}$

$\begin{array}{lllll}1 & 1 & 2 & 0 & 2\end{array}$

$\begin{array}{lllll}2 & 1 & 0 & 1 & 2\end{array}$

$\begin{array}{lllll}2 & 2 & 1 & 1 & 2\end{array}$

$\begin{array}{lllll}2 & 0 & 2 & 1 & 2\end{array}$

$\begin{array}{lllll}0 & 0 & 0 & 2 & 2\end{array}$

$\begin{array}{lllll}0 & 1 & 1 & 2 & 2\end{array}$

$\begin{array}{lllll}0 & 2 & 2 & 2 & 2\end{array}$ 
.108.

\subsection{Observaçōes}

a. A construção dos blocos na sërie $3^{\mathrm{n}}$ pode ser analoga aquela vista para a sërie $2^{n}$, ou seja, nos blocos $I$, II e III estão res pectivamente, as combinações de tratamentos com $0,1,2$ (mod. 3) 1etras em comum com $\mathrm{BC}^{2} \mathrm{D}_{\mathrm{O}}, \mathrm{BC}^{2} \mathrm{D}_{1}$ e $\mathrm{BC}^{2} \mathrm{D}_{2}$, respectivamente. Ou ainda, podemos construir o primeiro bloco procurando no experimento escolhido as comb $\underline{1}$ nações de tratamentos que satisfazem a $\mathrm{BC}^{2} \mathrm{D}_{\mathrm{O}}$, e colocando-as na "ordem padrão". Para construirmos o bloco II, selecionamos uma combinação de tratamento que satisfaz a $\mathrm{BC}^{2} \mathrm{D}_{1}$, e verificamos qual è o nümero modular que somado a primeira combinação de tratamento do bloco I dã a combinação de tratamento do bloco II. Por exemplo ab, nos dã o nümero modular 11000. Para construirmos o bloco III, selecionamos uma combinação de tratamento que satisfaz a $\mathrm{BC}^{2} \mathrm{D}_{2}$, $1 . \dot{\mathrm{e}} ., \mathrm{a}^{2} \mathrm{~b}^{2}$, e verificamos qual é o nümero modular que somado a primeira combinação de tratamento do bloco I, dã a combinação de tratamento do bloco III, no caso, o nümero modular é 22000 .

b. 0 Indice da interação a ser confundida com blocos, co mo tambëm o Indice da relação de identidade è prē-determinado, enquanto que os indices das interações generalizadas são determinados da seguinte maneira:

Seja a interação $Y=B^{2} \mathrm{D}$ para ser confundida com blocos, e a relação de identidade escolhida foi $I=\mathrm{AB}^{2} \mathrm{CDE}_{0}^{2}$, então temos: 


\section{b.1) Para o bloco I}

$$
\begin{gathered}
Y \times I=B C^{2} D_{0} \times A B^{2} C D E_{0}^{2}, \quad \text { ou } \\
x_{2}+2 x_{3}+x_{4}=0 \quad(\bmod .3) \\
\frac{x_{1}+2 x_{2}+x_{3}+x_{4}+2 x_{5}=0}{x_{1}+3 x_{2}+3 x_{3}+2 x_{4}+2 x_{5}=0} \quad(\bmod .3)
\end{gathered}
$$

seja: $A D^{2} E_{0}^{2}$

$$
\begin{aligned}
& \text { e } \quad \mathrm{Y} \times \mathrm{I}^{2}=\mathrm{BC}^{2} \mathrm{D}_{\mathrm{o}} \times\left(\mathrm{AB}^{2} \mathrm{CDE}_{\mathrm{o}}^{2}\right)^{2} \text {, ou } \\
& x_{2}+2 x_{3}+x_{4} \quad=0 \quad(\bmod .3) \Rightarrow x_{2}+2 x_{3}+x_{4} \quad=0 \\
& \Rightarrow 2\left(x_{1}+2 x_{2}+x_{3}+x_{4}+2 x_{5}\right)=2 \times 0(\bmod .3) \Rightarrow 2 x_{1}+4 x_{2}+2 x_{3}+2 x_{4}+4 x_{5}=0 \\
& 2 \mathrm{x}_{1}+2 \mathrm{x}_{2}+\mathrm{x}_{3}+0 \mathrm{x}_{4}+\mathrm{x}_{5}=0 \Longrightarrow \\
& \Rightarrow 2\left(2 x_{1}+2 x_{2}+x_{3}+x_{5}\right)=2 \times 0 \Rightarrow x_{1}+x_{2}+2 x_{3}+2 x_{5}=0(\bmod .3),
\end{aligned}
$$

ou seja: $A B C^{2} E_{0}^{2}$

\section{b.2) Para o bloco II:}

$$
\begin{aligned}
& \mathrm{Y} \times \mathrm{I}=\mathrm{BC}^{2} \mathrm{D}_{1} \times \mathrm{AB}^{2} \mathrm{CDE}_{\mathrm{o}}^{2}, \quad \text { ou } \\
& \mathrm{x}_{2}+2 \mathrm{x}_{3}+\mathrm{x}_{4}=1 \quad(\bmod .3) \\
& \mathrm{x}_{1}+2 \mathrm{x}_{2}+\mathrm{x}_{3}+\mathrm{x}_{4}+2 \mathrm{x}_{5}=0 \quad(\bmod .3) \\
& \mathrm{x}_{1}+0 \mathrm{x}_{2}+0 \mathrm{x}_{3}+2 \mathrm{x}_{4}+2 \mathrm{x}_{5}=1 \Rightarrow \mathrm{x}_{1}+2 \mathrm{x}_{4}+2 \mathrm{x}_{5}=1 \quad(\bmod .3) \text {, ou } \mathrm{AD}^{2} \mathrm{E}_{1}^{2}
\end{aligned}
$$




$$
\begin{aligned}
& \mathrm{Y} \times \mathrm{I}^{2}=\mathrm{BC}^{2} \mathrm{D}_{1} \times \quad\left(\mathrm{AB}^{2} \mathrm{CDE}_{\mathrm{o}}^{2}\right)^{2}, \quad \text { ou } \\
& \mathrm{x}_{2}+2 \mathrm{x}_{3}+\mathrm{x}_{4} \quad=1 \quad(\bmod .3) \Rightarrow \mathrm{x}_{2}+2 \mathrm{x}_{3}+\mathrm{x}_{4} \quad=1 \\
& 2\left(\mathrm{x}_{1}+2 \mathrm{x}_{2}+\mathrm{x}_{3}+\mathrm{x}_{4}+2 \mathrm{x}_{5}\right)=0 \quad(\bmod , 3) \Rightarrow 2 \mathrm{x}_{1}+\mathrm{x}_{2}+2 \mathrm{x}_{3}+2 \mathrm{x}_{4}+\mathrm{x}_{5}=0 \\
& 2 \mathrm{x}_{1}+2 \mathrm{x}_{2}+\mathrm{x}_{3}+0 \mathrm{x}_{4}+\mathrm{x}_{5}=1 \Rightarrow \\
& 2\left(2 \mathrm{x}_{1}+2 \mathrm{X}_{2}+\mathrm{X}_{3}+\mathrm{X}_{5}\right)=2 \times 1 \Rightarrow \mathrm{X}_{1}+\mathrm{X}_{2}+2 \mathrm{X}_{3}+2 \mathrm{X}_{5}=2 \text { (mod. 3), ou } \mathrm{ABC}^{2} \mathrm{E}_{2}^{2} \text {. }
\end{aligned}
$$

\section{b.3) Para o bloco III:}

$\mathrm{Y} \times \mathrm{I}=\mathrm{BC}^{2} \mathrm{D}_{2} \times \mathrm{AB}^{2} \mathrm{CDE}_{\mathrm{O}}^{2}, \quad$ ou

$$
\begin{aligned}
& \mathrm{x}_{2}+2 \mathrm{x}_{3}+\mathrm{x}_{4}=2 \quad(\bmod \cdot 3) \\
& \mathrm{x}_{1}+2 \mathrm{x}_{2}+\mathrm{x}_{3}+\mathrm{x}_{4}+2 \mathrm{x}_{5}=0 \quad(\bmod \cdot 3) \\
& \mathrm{x}_{1}+0 \mathrm{x}_{2}+0 \mathrm{x}_{3}+2 \mathrm{x}_{4}+2 \mathrm{x}_{5}=2 \Rightarrow \mathrm{x}_{1}+2 \mathrm{x}_{4}+2 \mathrm{x}_{5}=2(\bmod .3) \text {, ou } \mathrm{AD}^{2} \mathrm{E}_{2}^{2} \text {. } \\
& \mathrm{Y} \times \mathrm{I}^{2}=\mathrm{BC}^{2} \mathrm{D}_{2}+\left(\mathrm{AB}^{2} \mathrm{CDE}_{\mathrm{o}}^{2}\right)^{2}, \quad \mathrm{ou} \\
& \mathrm{x}_{2}+2 \mathrm{x}_{3}+\mathrm{x}_{4}=2 \quad(\bmod .3) \Rightarrow \mathrm{x}_{2}+2 \mathrm{x}_{3}+\mathrm{x}_{4} \quad=2(\bmod , 3) \\
& 2\left(x_{1}+2 x_{2}+x_{3}+x_{4}+2 x_{5}\right)=2 \times 0 \quad(\bmod .3) \Rightarrow \frac{1+x_{2}+2 x_{3}+2 x_{4}+x_{5}=0}{2 x_{1}+2 x_{2}+x_{3}+0 x_{4}+x_{5}=2} \Rightarrow \text { (mod. 3) } \\
& 2\left(2 \mathrm{x}_{1}+2 \mathrm{x}_{2}+\mathrm{x}_{3}+\mathrm{x}_{5}\right)=2 \times 2 \Rightarrow \mathrm{x}_{1}+\mathrm{x}_{2}+2 \mathrm{x}_{3}+2 \mathrm{x}_{5}=1 \quad(\bmod .3), \quad \text { ou } \\
& A B C^{2} E_{1}^{2}
\end{aligned}
$$

0 esboço do quadro da anālise de variância para este experimento $\left(3^{5-1}\right)$ em 3 blocos é: 
C. Variação

Blocos

Efeitos principais

Interações de 2 fatores

Residuo
G.L.

\subsubsection{Método prático de Yates}

YATES (1937) desenvolveu um método geral para calcular os estimadores dos efeitos principais e das interações, e suas correspondentes somas de quadrados, para um ensaio fatorial $2^{\mathrm{n}}$, sem escrever especificamente suas expressões algëbricas. Este método tambēm pode ser generalizado para fatoriais com mais de 2 níveis por fator.

$$
\text { Para aplicar este método devemos proceder da seguinte }
$$

maneira:

a. As combinações de tratamentos são colocadas em uma "ordem padrão", ou seja, cada fator è introduzido por vez e seguido por todas combinações desse fator com os outros. A seguir substitutimos es sas combinações de tratamentos pelas suas respectivas produções.

$$
\text { b. Os quadros consistem de colunas numeradas de } 1 \text { até o }
$$

total de fatores. 
c. A primeira parte da coluna (1) é formada pela soma dos $2^{\mathrm{n}-1}$ parea de nümeros da coluna produção, enquanto a segunda parte é formada pela subtração dos mesmos $2^{\mathrm{n}-1}$ pares, em cada caso o nümero superior è subtraido do inferior.

d. A coluna (2) è formada da mesma maneira, tomando como base a coluna (1). Este processo è repetido até a coluna n.

e. Na coluna ( $n$ ) temos o total, e os totais dos efeitos principais e das interações, os quais estão identificados na coluna $(n+1)$.

f. Na coluna $(n+2)$ temos a correção, e as $S Q^{\prime S}$ de todos efeitos principais e interações. Para chegarmos as estas $\mathrm{SQ}^{\prime}{ }^{\prime}$, basta elevarmos ao quadrado os números da coluna (n) e dividirmos por $r 2^{n}$.

Exemplo: Vamos tomar como exemplo o fatorial $2^{3}$, para enfocar o método prático de YATES. Então temos:

\begin{tabular}{|c|c|c|c|c|}
\hline $\begin{array}{l}\text { TRATA- } \\
\text { MENTOS } \\
\end{array}$ & (1) & (2) & (3) & $\begin{array}{l}\text { IDENTICA } \\
\text { ÇÃO }(4)\end{array}$ \\
\hline $\begin{array}{l}(1) \\
\mathrm{a} \\
\mathrm{b} \\
\mathrm{ab} \\
\mathrm{c} \\
\mathrm{ac} \\
\mathrm{bc} \\
\mathrm{abc}\end{array}$ & $\begin{array}{l}(1)+a \\
b+a b \\
c+a c \\
b c+a b c \\
a-(1) \\
a b-b \\
a c-c \\
a b c-b c\end{array}$ & $\begin{array}{l}(1)+a+b+a b \\
c+a c+b c+a b c \\
a-(1)+a b-b \\
a c-c+a b c-b c \\
b+a b-(1)-a \\
b c+a b c-c-a c \\
a b-b-a+(1) \\
a b c-b c-a c+c\end{array}$ & $\begin{array}{l}(1)+a+b+a b+c+a c+b c+a b c \\
a-(1)+a b-b+a c-c+a b c-b c \\
b+a b-(1)-a+b c+a b c-c-a c \\
a b-b-a+(1)+a b c-b c-a c+c \\
c+a c+b c+a b c-(1)-a-b-a b \\
a c-c+a b c-b c-a+1-a b+b \\
b c+a b c-c-a c-b-a b+(1)+a \\
a b c-b c-a c+c-a b+b+a-(1)\end{array}$ & $\begin{array}{l}\text { G } \\
A \\
B \\
A B \\
C \\
A C \\
B C \\
A B C\end{array}$ \\
\hline
\end{tabular}




\subsection{Material}

Os dados foram gerados atravès do processo de simulação, tomando como exemplo o fatorial $2^{6}$, obedecendo a seguinte metodologia:

a. 0 modelo matemático usado foi o seguinte:

$$
\begin{aligned}
& \mathrm{Y}_{i j k \ell \mathrm{mn}}=\mu+\mathrm{A}_{i}+\mathrm{B}_{j}+\mathrm{AB}_{i j}+\mathrm{C}_{k}+\mathrm{AC}_{i k}+\mathrm{BC}_{j k}+\mathrm{ABC}_{i j k}+\mathrm{D}_{\ell}+\mathrm{AD}_{1 \ell}+\mathrm{BD}_{j \ell}+\mathrm{ABD}_{1 j \ell}+\mathrm{CD}_{k \ell}+ \\
& +\mathrm{ACD}_{i k \ell}+\mathrm{BCD}_{j k \ell}+\mathrm{ABCD}_{i j k \ell}+\mathrm{E}_{\mathrm{m}}+\mathrm{AE}_{i \mathrm{~m}}+\mathrm{BE}_{j \mathrm{~m}}+\mathrm{ABE}_{i j \mathrm{~m}}+\mathrm{CE}_{\mathrm{km}}+\mathrm{ACE} \mathrm{ikm}+ \\
& +\mathrm{BCE}_{j \mathrm{~km}}+\mathrm{ABCE}_{1 j \mathrm{~km}}+\mathrm{DE} \mathrm{E}_{\ell \mathrm{m}}+\mathrm{ADE} \mathrm{ilm}_{1 \mathrm{~m}}+\mathrm{BDE}_{j \ell \mathrm{m}}+\mathrm{ABDE}_{1 \mathrm{j} \ell \mathrm{m}}+\mathrm{CED}_{\mathrm{k} \ell \mathrm{m}}+ \\
& +\mathrm{ACDE}_{i k \ell \mathrm{m}}+\mathrm{BCDE} \mathrm{jk \ell m}+\mathrm{ABCDE} \mathrm{ijk \ell m}+\mathrm{AF}_{\mathrm{in}}+\mathrm{BF}_{\mathrm{jn}}+\mathrm{ABF} \mathrm{ijn}_{\mathrm{kn}}+\mathrm{CF}_{\mathrm{k}}+\mathrm{ACF}_{\mathrm{ikn}}+
\end{aligned}
$$

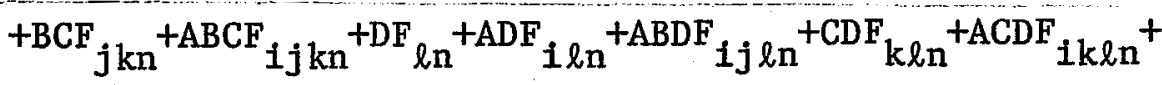

$$
\begin{aligned}
& +\mathrm{BCDF}_{j k \ell \mathrm{n}}+\mathrm{ABCDF}_{i j k \ell \mathrm{n}}+\mathrm{EF}_{\mathrm{mn}}+\mathrm{AEF}_{i m n}+\mathrm{BEF}_{j \mathrm{mn}}+\mathrm{ABEF}_{i j \mathrm{mn}}+\mathrm{CEF}_{\mathrm{kmn}}+
\end{aligned}
$$

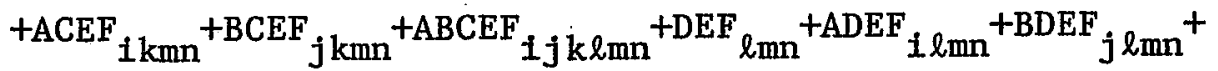

$$
\begin{aligned}
& +\mathrm{ABDEF}_{i j \ell \mathrm{mn}}+\mathrm{CDEF}_{\mathrm{k} \ell \mathrm{mn}}+\mathrm{ACDEF}_{1 \mathrm{k} \ell \mathrm{mn}}+\mathrm{BCDEF}_{\mathrm{j} k \ell \mathrm{mn}}{ }^{+} \\
& { }^{+A B C D E F_{i j k \ell m n}}+e_{i j k \ell m n}
\end{aligned}
$$

As restrições do modelo foram:

a.1) $\sum_{i} A B_{i j}=\sum_{j} A B_{i j}=\sum_{i} A C_{i k}=\ldots=\sum_{n} E F_{m n}=0$

a.2) $\sum_{i j} A B C_{i j k}=\sum_{i j} A B C_{i j k}=\ldots=\sum_{m n} D_{\ell m n}=0$

a.3) $\sum_{i j k} \mathrm{ABCD}_{i j k \ell}=\sum_{i k \ell} \mathrm{ABCD}_{1 j k \ell}=\ldots \ldots \ldots=0$

a.4) $\sum_{i j k \ell} \mathrm{ABCDE}_{i j k \ell m}=\sum_{i k \ell m} \mathrm{ABCDE}_{i j k \ell m}=\ldots=0$ 
a.5) $\sum_{i j k \ell m} A B C D E F_{i j k \ell m n}=\sum_{i k \ell m n} A B C D E_{i j k \ell m n}=\ldots=0$

onde, $i=j=k=l=m=n=1,2$

b. A média geral, os efeitos principais e as interações foram fixadas, tendo os seguintes valores:

$$
\begin{aligned}
& \mu=100 \\
& \mathrm{~A}_{1}=\mathrm{B}_{1}=\mathrm{C}_{1}=\mathrm{D}_{1}=\mathrm{E}_{1}=\mathrm{F}_{1}=0 \\
& A_{2}=90 ; B_{2}=80 ; C_{2}=75 ; D_{2}=70 ; E_{2}=65 ; F_{2}=60 \\
& A_{1} B_{1}=A_{1} C_{1}=A_{1} D_{1}=A_{1} E_{1}=A_{1} F_{1}=B_{1} C_{1}=B_{1} D_{1}=B_{1} E_{1}=B_{1} F_{1}=C_{1} D_{1}=C_{1} E_{1}=C_{1} F_{1}=D_{1} E_{1}=D_{1} F_{1}=E_{1} F_{1}=6 \\
& A_{1} B_{2}=A_{1} C_{2}=A_{1} D_{2}=A_{1} E_{2}=A_{1} F_{2}=B_{1} C_{2}=B_{1} D_{2}=B_{1} E_{2}=B_{1} F_{2}=C_{1} D_{2}=C_{1} E_{2}=C_{1} F_{2}=D_{1} E_{2}=D_{1} F_{2}=E_{1} F_{1}=-6 \\
& A_{2} B_{1}=A_{2} C_{1}=A_{2} D_{1}=A_{2} E_{1}=A_{2} F_{1}=B_{2} C_{1}=B_{2} D_{1}=B_{2} E_{1}=B_{2} F_{1}=C_{2} D_{1}=C_{2} E_{1}=C_{2} F_{1}=D_{2} E_{1}=D_{2} F_{1}=E_{2} F_{1}=-4 \\
& A_{2} B_{2}=A_{2} C_{2}=A_{2} D_{2}=A_{2} E_{2}=A_{2} F_{2}=B_{2} C_{2}=B_{2} D_{2}=B_{2} E_{2}=B_{2} F_{2}=C_{2} D_{2}=C_{2} E_{2}=C_{2} F_{2}=D_{2} E_{2}=D_{2} F_{2}=E_{2} F_{2}=4 \\
& A_{1} B_{1} C_{k}=A_{1} B_{1} D_{\ell}=A_{1} B_{1} E_{m}=A_{1} B_{1} F_{n}=A_{1} C_{1} D_{\ell}=A_{1} C_{1} E_{m}=A_{1} C_{1} F_{n}=A_{1} D_{1} E_{m}=A_{1} D_{1} F_{n}=A_{1} E_{1} F_{n}= \\
& =B_{1} C_{1} D_{l}=B_{1} C_{1} E_{m}=B_{1} C_{1} F_{n}=B_{1} D_{1} E_{m}=B_{1} D_{1} F_{n}=B_{1} E_{1} F_{n}=C_{1} D_{1} E_{m}=C_{1} D_{1} F_{n}=C_{1} E_{1} F_{n}=D_{1} E_{1} F_{n}=1,0 \\
& A_{1} B_{2} C_{k}=A_{1} B_{2} D_{\ell}=A_{1} B_{2} E_{m}=A_{1} B_{2} F_{n}=A_{1} C_{2} D_{\ell}=A_{1} C_{2} E_{m}=A_{1} C_{2} F_{n}=A_{1} D_{2} E_{m}=A_{1} D_{2} F_{n}=A_{1} E_{2} F_{n}= \\
& =B_{1} C_{2} D_{\ell}=B_{1} C_{2} E_{m}=B_{1} C_{2} F_{n}=B_{1} D_{2} E_{m}=B_{1} D_{2} F_{n}=B_{1} E_{2} F_{n}=C_{1} D_{2} E_{m}=C_{1} D_{2} F_{n}=C_{1} E_{2} F_{n}=D_{1} E_{2} F_{n}=-1,0 \\
& A_{2} B_{1} C_{k}=A_{2} B_{1} D_{\ell}=A_{2} B_{1} E_{m}=A_{2} B_{1} F_{n}=A_{2} C_{1} D_{\ell}=A_{2} C_{1} E_{m}=A_{2} C_{1} F_{n}=A_{2} D_{1} E_{m}=A_{2} D_{1} F_{n}=A_{2} E_{1} F_{n}= \\
& =B_{2} C_{1} D_{\ell}=B_{2} C_{1} E_{m}=B_{2} C_{1} F_{n}=B_{2} D_{1} E_{m}=B_{2} D_{1} F_{n}=B_{2} E_{1} F_{n}=C_{2} D_{1} E_{m}=C_{2} D_{1} F_{n}=C_{2} E_{1} F_{n}=D_{2} E_{1} F_{n}=-0,5 \\
& A_{2} B_{2} C_{k}=A_{2} B_{2} D_{\ell}=A_{2} B_{2} E_{m}=A_{2} B_{2} F_{n}=A_{2} C_{2} D_{\ell}=A_{2} C_{2} E_{m}=A_{2} C_{2} F_{n}=A_{2} D_{2} E_{m}=A_{2} D_{2} F_{n}=A_{2} E_{2} F_{n}= \\
& =B_{2} C_{2} D_{\ell}=B_{2} C_{2} E_{m}=B_{2} C_{2} F_{n}=B_{2} D_{2} E_{m}=B_{2} D_{2} F_{n}=B_{2} E_{2} F_{n}=C_{2} D_{2} E_{m}=C_{2} D_{2} F_{n}=C_{2} E_{2} F_{n}=D_{2} E_{2} F_{n}=0,5
\end{aligned}
$$




$$
\begin{aligned}
& A_{1} B_{1} C_{k} D_{\ell}=A_{1} B_{1} C_{k} E_{m}=A_{1} B_{1} C_{k} F_{n}=A_{1} B_{1} D_{\ell} E_{m}=A_{1} B_{1} D_{\ell} F_{n}=A_{1} B_{1} E_{m} F_{n}=A_{1} C_{1} D_{\ell} E_{m}=A_{1} C_{1} D_{\ell} F_{n}= \\
& =A_{1} C_{1} E_{m} F_{n}=A_{1} D_{1} E_{m} F_{n}=B_{1} C_{1} D_{\ell} E_{m}=B_{1} C_{1} D_{\ell} F_{n}=B_{1} C_{1} E_{m} F_{n}=B_{1} D_{1} E_{m} F_{n}=C_{1} D_{1} E_{m} F_{n}=0,5 \\
& A_{1} B_{2} C_{k} D_{\ell}=A_{1} B_{2} C_{k} E_{m}=A_{1} B_{2} C_{k} F_{n}=A_{1} B_{2} D_{\ell} E_{m}=A_{1} B_{2} D_{\ell} F_{n}=A_{1} B_{2} E_{m} F_{n}=A_{1} C_{2} D_{\ell} E_{m}=A_{1} C_{2} D_{\ell} F_{n}= \\
& \Rightarrow A_{1} C_{2} E_{m} F_{n}=A_{1} D_{2} E_{m} F_{n}=B_{1} C_{2} D_{\ell} E_{m}=B_{1} C_{2} D_{\ell} F_{n}=B_{1} C_{2} E_{m} F_{n}=B_{1} D_{2} E_{m} F_{n}=C_{1} D_{2} E_{m} F_{n}=-0,5
\end{aligned}
$$$$
A_{2} B_{1} C_{k} D_{\ell}=A_{2} B_{1} C_{k} E_{m}=A_{2} B_{1} C_{k} F_{n}=A_{2} B_{1} D_{\ell} E_{m}=A_{2} B_{1} D_{\ell} F_{n}=A_{2} B_{1} E_{m} F_{n}=A_{2} C_{1} D_{\ell} E_{m}=A_{2} C_{1} D_{\ell} F_{n}=
$$$$
=A_{2} C_{1} E_{m} F_{n}=A_{2} D_{1} E_{m} F_{n}=B_{2} C_{1} D_{\ell} E_{m}=B_{2} C_{1} D_{\ell} F_{n}=B_{2} C_{1} E_{m} F_{n}=B_{2} D_{1} E_{m} F_{n}=C_{2} D_{1} E_{m} F_{n}=-0,5
$$

$$
\begin{aligned}
& A_{2} B_{2} C_{k} D_{\ell}=A_{2} B_{2} C_{k} E_{m}=A_{2} B_{2} C_{k} F_{n}=A_{2} B_{2} D_{\ell} E_{m}=A_{2} B_{2} D_{\ell} F_{n}=A_{2} B_{2} E_{m} F_{n}=A_{2} C_{2} D_{\ell} E_{m}=A_{2} C_{2} D_{\ell} F_{n} \\
& =A_{2} C_{2} E_{m} F_{n}=A_{2} D_{2} E_{m} F_{n}=B_{2} C_{2} D_{\ell} E_{m}=B_{2} C_{2} D_{\ell} F_{n}=B_{2} C_{2} E_{m} F_{n}=B_{2} D_{2} E_{m} F_{n}=C_{2} D_{2} E_{m} F_{n}=0,5
\end{aligned}
$$$$
A_{1} B_{1} C_{k} D_{\ell} E_{m}=A_{1} B_{1} C_{k} D_{\ell} F_{n}=A_{1} B_{1} C_{k} E_{m} F_{m}=A_{1} B_{1} D_{\ell} E_{m} F_{n}=A_{1} C_{1} D_{\ell} E_{m} F_{n}=B_{1} C_{1} D_{\ell} E_{m} F_{n}=0,2
$$$$
A_{1} B_{2} C_{k} D_{\ell} E_{m}=A_{1} B_{2} C_{k} D_{\ell} F_{n}=A_{1} B_{2} C_{k} E_{m} F_{n}=A_{1} B_{2} D_{\ell} E_{m} F_{n}=A_{1} C_{2} D_{\ell} E_{m} F_{n}=B_{1} C_{2} D_{\ell} E_{m} F_{n}=-0,2
$$$$
A_{2} B_{1} C_{k} D_{\ell} E_{m}=A_{2} B_{1} C_{k} D_{\ell} F_{n}=A_{2} B_{1} C_{k} E_{m} F_{n}=A_{2} B_{1} D_{\ell} E_{m} F_{n}=A_{2} C_{1} D_{\ell} E_{m} F_{n}=B_{2} C_{1} D_{\ell} E_{m} F_{n}=-0,2 .
$$

$$
A_{2} B_{2} C_{k} D_{\ell} E_{m}=A_{2} B_{2} C_{k} D_{\ell} F_{n}=A_{2} B_{2} C_{k} E_{m} F_{n}=A_{2} B_{2} D_{\ell} E_{m} F_{n}=A_{2} C_{2} D_{\ell} E_{m} F_{n}=B_{2} C_{2} D_{\ell} E_{m} F_{n}=0,2
$$

$$
A_{1} B_{1} C_{k} D_{\ell} E_{m} F_{n}=0,2 ; A_{1} B_{2} C_{k} D_{\ell} E_{m} F_{n}=-0,2 ; A_{2} B_{1} C_{k} D_{\ell} E_{m} F_{n}=-0,2 ; A_{2} B_{2} C_{k} D_{\ell} E_{m} F_{n}=0,2
$$

\section{c) Os erros foram tirados de uma tabela com distribuição}

normal com $\mu=0$ e $\sigma=1$, então temos:

$$
\begin{array}{llll}
e_{111111}=-0,288 & e_{111121}=1,620 & e_{111112}=1,045 & e_{111122}=-0,538 \\
e_{211111}=1,090 & e_{211121}=-1,041 & e_{211112}=-0,528 & e_{211122}=-1,094 \\
e_{121111}=0,785 & e_{121121}=-1,698 & e_{121112}=-1,433 & e_{121122}=1,298
\end{array}
$$


.116 .

\begin{tabular}{|c|c|c|c|c|c|}
\hline 221111 & $=0$ & $e_{221121}$ & $=1$ & $e_{221112}=2$ & $e_{221122}=$ \\
\hline 12111 & $=1$, & $e_{112121}$ & $=0,032$ & $e_{112112}=-1,190$ & $e_{112122}=-c$ \\
\hline 212111 & $=-0$, & $e_{212121}$ & $=0,1$ & $e_{212112}=0,192$ & $\mathrm{e}_{212122}=1,192$ \\
\hline 11 & $=0,187$ & $e_{122121}$ & $=0,290$ & $e_{122112}=0$, & $e_{122122}=0,412$ \\
\hline 11 & $=-0,457$ & $e_{222121}$ & $=0,873$ & $e_{222112}=1,216$ & $\mathrm{e}_{222122}=0,161$ \\
\hline 11 & $=0,448$ & $e_{111221}$ & $=-0,289$ & $e_{111212}=1,705$ & $e_{111222}=-0,631$ \\
\hline . & $=0,960$ & $e_{211221}$ & $=1,119$ & $e_{211212}=-0,145$ & $e_{211222}=0,748$ \\
\hline 211 & $=-0,491$ & $e_{121221}$ & $=-0,792$ & $e_{121212}=-0,066$ & $e_{121222}=-0,218$ \\
\hline$e_{221211}$ & $=0,219$ & $\mathrm{e}_{22}$ & $=0,063$ & $e_{221212}=1,810$ & $e_{221222}=-1,530$ \\
\hline 11 & $=-0,16$ & $221=$ & $=0$ & $e_{112212}=-0$ & $e_{112222}=$ \\
\hline & $=1,090$ & $e_{212221}=$ & $=0,427$ & $e_{212212}=0,484$ & $e_{212222}=$ \\
\hline 211 & $=1,239$ & $2221=$ & $=0,084$ & $e_{122212}=1,458$ & $e_{122222}=0$, \\
\hline & & $22221=$ & $=-0,086$ & $e_{222212}=$ & $e_{222222}=$ \\
\hline
\end{tabular}

d) Variamos o desvio padrão dos erros e assim estruturamos 4 experimentos, isto é:

Experimento 1: Com erros normais com $\mu=0$ e $\sigma=5$

Experimento 2: Com erros normais $\operatorname{com} \mu=0$ e $\sigma=10$

Experimento 3: Com erros normais $\operatorname{com} \mu=0$ e $\sigma=15$

Experimento 4: Com erros normais $\operatorname{com} \mu=0$ e $\sigma=20$ 
.117.

\section{RESULTADOS E DISCUSSÁO}

Foram realizadas anālises de variāncia para o

fatorial completo com e sem confundimento como também para o fatorial fracionado para os 4 casos estudados.

Para isso foi utilizado o método prático de Yates, para calcularmos as $S^{\prime}$ 's referentes a esses experimen tos.

Passaremos a anälise e comentar os resultados desses 4 experimentos. Para estes experimentos vamos considerar que as interações de 3 ou mais fatores são insignifi cantes, e assim as mesmas compõem o residuo. 


\subsection{EXPERIMENTO 1}

A partir do Quadro 1 (ver anexo 1), obteve-se o seguinte quadro de anälise de variância para o fatorial comple to:

\section{Variação \\ G.L . \\ S.Q. \\ $21491.328,9$ \\ Tratamentos}

A

B

C

D

E

F

$\mathrm{AB}$

A C

$\mathrm{AD}$

$\mathrm{AE}$

$\mathrm{AF}$

BC

BD

BE

B F

CD

CE

CF

DE

DF

EF

Residuo

Total
$1 \quad 133.099,11$

1

$$
93.177,563
$$

$75.218,548$

$59.755,191$

$44.421,358$

$39.321,898$

$9.079,2312$

$5.068,3721$

$3.345,321$

$2.210,9979$

1.767\%,3616

$5.917,4556$

$2.658,8203$

$1.946,4641$

$2.334,0977$

$3.085,9414$

2. 596,5394

1. 543,3112

$1.898,5628$

$1.630,0397$

$1.252,7175$

$1.114,3453$
Q.M.

F 
os valores de $F$ por interpolação harmōnica

são:

$$
\begin{aligned}
& F_{5 \%}(1,42)=4,07 \\
& F_{1 \%}(1,42)=7,28
\end{aligned}
$$

Como vemos tanto os efeitos principais como as interações de 2. fatores foram altamente significativos tanto ao nivel de $5 \%$ como ao nivel de $1 \%$.

Agora vamos confundir com efeitos de blocos a interação de ordem mais elevada, ou seja ABCDEF, assim temos os seguintes blocos.

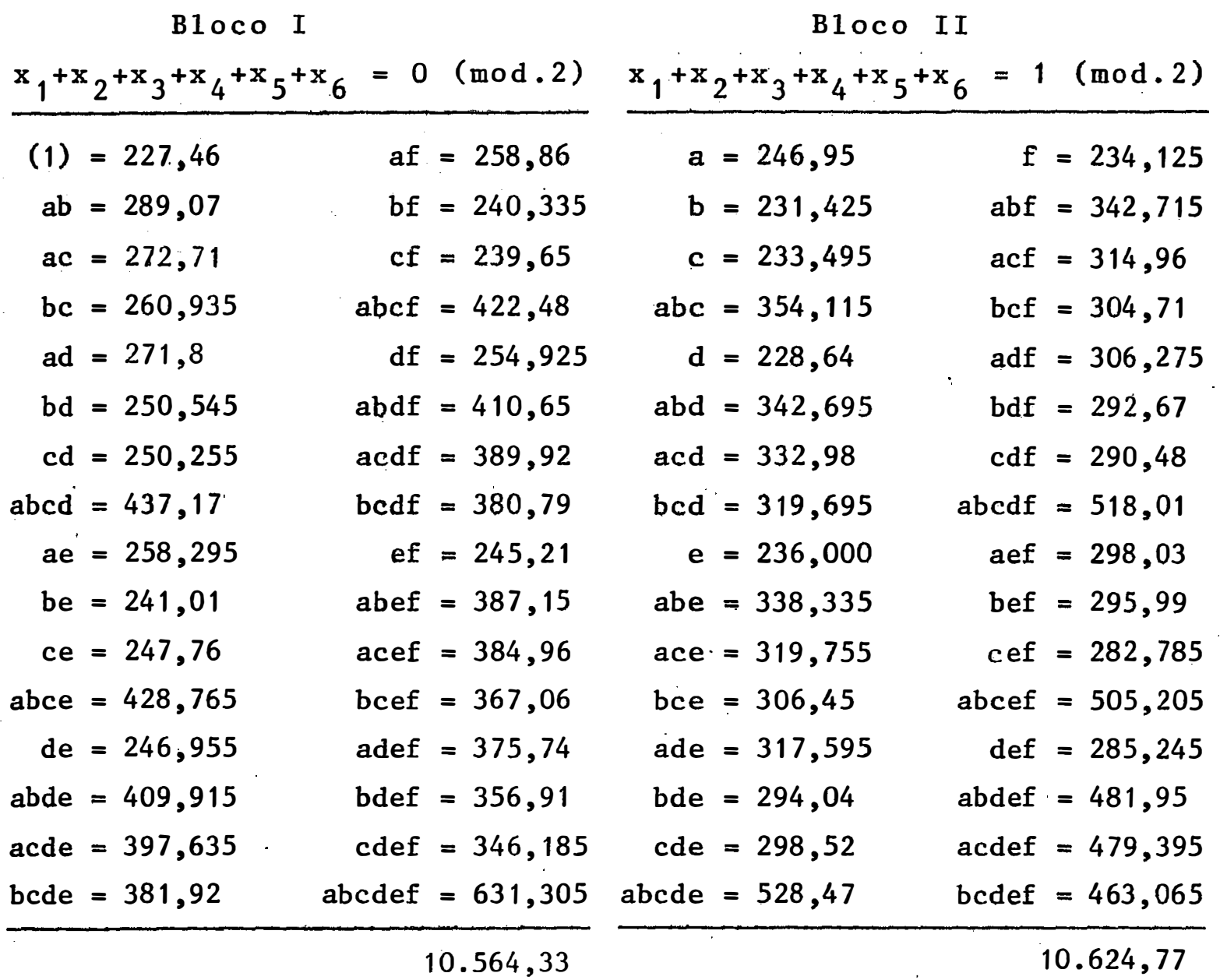


Como a interação ABCDEF está confundida, então a $S Q_{B 1 o c o s}=S Q_{A B C D E F}=57,078025$, e a $S Q_{\operatorname{Res}} \mathrm{c} / \mathrm{conf}$ $=\mathrm{SQ}_{\text {Res s/conf }}-\mathrm{SQ}_{\mathrm{ABCDEF}}$, como também se perde $1 \mathrm{~g} .1$. no residuo. 0 quadro da anālise de variāncia será:

\section{Variação \\ B1ocos \\ G.L. \\ S.Q. \\ 57,078025}

Tratamentos

A

B

C

D

E

F

$\mathrm{AB}$

AC

AD

AE

AF

BC

BD

BE

BF

CD

CE

CF

$\mathrm{DE}$

DF

EF

Residuo

Tota 1
Q.M.

$.21491 .328,9$

$1133.099,11$

$93.177,563$

$75.218,548$

$59.755,191$

$44.421,358$

$39.321,898$

$9.079,2312$

$5.068,3721$

$3.345,321$

2. 210,9979

$1.767,3616$

$5.917,4556$

$2.658,8203$

$1.946,4641$

$2.334,0977$

$3.085,9414$

$2.596,5394$

$1.543,3112$

$1.898,5628$

$1.630,0397$

$1.252,7175$

$1.057,2673$
$133.099,11$

$93.177,563$

$75.218,548$

$59.755,191$

$44.421,358$

$39.321,898$

$9.079,2312$

$5.068,3721$

$3.345,321$

$2.210,9979$

1. 767,3616

$5.917,4556$

$2.658,8203$

$1.946,4641$

$2.334,0977$

$3.085,9414$

$2.596,5394$

$1.543,3112$

$1.898,5628$

$1.630,0397$

$1.252,7175$

25,787007
$5.161,48 * *$

$3.613,35 * *$

$2.916,92 * *$

$2.317,26 * *$

1. $722,62 * *$

$1.524,87 * *$

$352,08 * *$

$196,55 * *$

$129,73 * *$

$85,74 * *$

$68,54 * *$

229,47 **

103,11 **

$75,48 * *$

$90,51 * *$

119,67 **

$100,69 *$ *

$59,85 * *$

$73,62 * *$

$63,21 * *$

$48,58 * *$ 
os valores de F por interpolação harmônica se

rão:

$$
\begin{aligned}
& F_{5 \%}(1,41)=4,074 \\
& F_{1 \%}(1,41)=7,29
\end{aligned}
$$

Podemos observar que:

a. Os efeitos principais e as interações de 2 fatores continuam sendo altamente significativos.

b. O QM do residuo com confundimento fica menor do que o QM: do residuo sem confundimento, esta diferença è de 0,739779 .

c. Esta diferença entre os QM's serā maior ou menor devido a escolha da interaça a ser confundida, por exemplo, se escolhessemos a interação ABEF, o Q.M. do residuo seria 21,433199. Mas, se fosse escolhida a interaça ACDF, O QM d do residuo seria 27,175372, como vemos o mesmo aumentou em relação ao $Q M$ do residuo do fatorial completo."

$$
\begin{aligned}
& \text { d. A eficiência serā: } \\
& \overline{\mathrm{E}}=\frac{\mathrm{n}_{\mathrm{b}} Q M_{B 1 \text { ocos }}+\mathrm{n}_{\mathrm{e}} \mathrm{QM}_{\text {erro }}}{\therefore\left(\mathrm{n}_{\mathrm{b}}+\mathrm{n}_{\mathrm{e}}\right) Q M_{\text {erro }}} \text {, onde }
\end{aligned}
$$




$$
\begin{aligned}
n_{b} & =1 \\
n_{e} & =63-1=62, \text { então: } \\
\hat{E} & =\frac{1 \times 57,078025+62 \times 25,787007}{63 \times 25,787007}=\frac{1.655,8725}{1.624,5814} \rightarrow \widehat{E}=1,019
\end{aligned}
$$

e. Se a interação escolhida fosse $A B E F$,

eficiência seria:

$$
\begin{gathered}
Q_{B 1 \text { ocos }}=235,58413 \\
Q_{\text {erro }}=\frac{878,76117}{41}=21,433199 \\
\widehat{E}=\frac{1 \times 235,58413+62 \times 21,433199}{63 \times 21,433199}=\frac{1.564,4425}{1.350,2915}=\bar{E}=1,159
\end{gathered}
$$

Comparando as duas eficiências acima, vemos que a interação ABEF quando confundida, nos dã uma eficiência maior do que a interação $A B C D E F$.

Agora vamos confundir as interações $A B D E$ e ACDF, como sabemos a interação generalizada BCEF também esta rá confundida, então temos os seguintes blocos: 
BLOCO I $\begin{array}{lll}x_{1}+x_{2}+x_{4}+x_{5} & =0 & 0 \\ x_{1}+x_{3}+x_{4}+x_{6} & =0 & (\bmod .2)\end{array}$

$$
\begin{array}{rlrl}
(1) & =227,46 \\
\text { abc } & =354,115 \\
\text { ad } & =271,8 & \text { abf } & =342,715 \\
\text { bcd } & =319,695 \\
\text { be } & =241,01 & \text { cf } & =239,65 \\
\text { ace } & =319,755 & \text { acdf } & =389,92 \\
\text { abde } & =409,915 & \text { aef } & =298,03 \\
\text { cde } & =298,52 \text { abcdef } & =367,06 & =631,305 \\
& & &
\end{array}
$$

BLOCO III

$x_{1}+x_{2}+x_{4}+x_{5}=1(\bmod \cdot 2)$

$$
x_{1}+x_{3}+x_{4}+x_{6}=0(\bmod .2)
$$

$$
\begin{array}{rlrl}
\mathrm{b} & =231,425 & \text { af } & =258,86 \\
\mathrm{ac} & =272,71 & \text { bcf } & =304,71 \\
\mathrm{abd} & =342,695 & \text { df } & =254,925 \\
\mathrm{~cd} & =250,255 \text { abcdf }=518,01 \\
\mathrm{e} & =236,000 & \text { abef } & =387,15 \\
\text { abce } & =428,765 & \text { cef } & =282,785 \\
\text { ade } & =317,595 & \text { bdef } & =356,91 \\
\text { bcde } & =381,92 & \text { acdef } & =479,395
\end{array}
$$$$
5.304,11
$$

BLOCO II $x_{1}+x_{2}+x_{4}+x_{5}=0 \quad(\bmod .2)$ $x_{1}+x_{3}+x_{4}+x_{6}=1(\bmod .2)$

\begin{tabular}{rlrl}
$a b$ & $=289,07$ & $f$ & $=234,125$ \\
$c$ & $=233,495$ & abcf & $=422,48$ \\
$b d$ & $=250,545$ & adf & $=306,275$ \\
acd & $=332,98$ & bcdf & $=380,79$ \\
ae & $=258,295$ & bef & $=295,99$ \\
bce & $=306,45$ & acef & $=384,96$ \\
de & $=246,955$ & abdef & $=481,95$ \\
abcde & $=528,47$ & cdef & $=346,185$ \\
\hline & & & $5.299,015$
\end{tabular}

$$
\begin{array}{r}
\text { BLOCO IV } \\
x_{1}+x_{2}+x_{4}+x_{5}=1(\bmod \cdot 2) \\
x_{1}+x_{3}+x_{4}+x_{6}=1(\bmod .2)
\end{array}
$$

$$
\begin{array}{rlrl}
\mathrm{a} & =246,95 & \text { bf } & =240,335 \\
\mathrm{bc} & =260,935 & \text { acf } & =314,96 \\
\mathrm{~d} & =228,64 & \text { abdf } & =410,65 \\
\text { abcd } & =437,17 & \text { cdf } & =290,48 \\
\text { abe } & =338,335 & \text { ef } & =245,21 \\
c e & =247,76 & \text { abcef } & =505,205 \\
\text { bde } & =294,04 \\
\text { acde } & =397,635 & \text { adef } & =375,74 \\
\text { bcdef } & =463,065
\end{array}
$$

Como as interações ABDE, ACDF e BCEF estão confundidas então a $\mathrm{SQ}_{\mathrm{B} \text { iocos }}=\mathrm{SQ}_{\mathrm{ABDE}}+\mathrm{SQ}_{\mathrm{ACDF}}+\mathrm{SQ}_{\mathrm{BCEF}}=$ $=2,7805563+0,155039+4,5956641 \rightarrow \mathrm{SQ}_{\mathrm{B} 1 \text { ocos }}=7,5312594$, então 
$\mathrm{SQ}_{\operatorname{Res} \mathrm{c} / \mathrm{conf}}=\mathrm{SQ}_{\operatorname{Res} \mathrm{s} / \mathrm{conf}}-\mathrm{SQ}_{\mathrm{B} \text { locos }}=1.114,3453-$ $7,5312594 \rightarrow \mathrm{SQ}_{\mathrm{Res}} \mathrm{c} / \mathrm{conf}=1.106,984$ Assim, temos o quadro da anälise de variância.

\begin{tabular}{lrr} 
C. Variação & G.L. & S.Q \\
\hline Blocos & 3 & 7,5 \\
Tratamentos & 21 & $491.328,9$
\end{tabular}

\begin{tabular}{crcccc} 
A & 1 & $133.099,11$ & $133.099,11$ & $4.689,92 * *$ \\
B & 1 & $93.177,563$ & $93.177,563$ & $3.282,23 * *$ \\
C & 1 & $75.218,548$ & $75.218,548$ & $2.650,42 * *$ \\
D & 1 & $59.755,191$ & $59.755,191$ & $2.105,55 * *$ \\
E & 1 & $44.421,358$ & $44.421,358$ & $1.565,24 * *$ \\
F & 1 & $39.321,898$ & $39.321,898$ & $1.385,56 * *$ \\
AB & 1 & $9.079,2312$ & $9.079,2312$ & $319,92 * *$ \\
AC & 1 & $5.068,3721$ & $5.068,3721$ & $178,59 * *$ \\
AD & 1 & $3.345,321$ & $3.345,321$ & $117,88 * *$ \\
AE & 1 & $2.210,9979$ & $2.210,9979$ & $77,91 * *$ \\
AF & 1 & $1.767,3616$ & $1.767,3616$ & $62,27 * *$ \\
BC & 1 & $5.917,4556$ & $5.917,4556$ & $208,51 * *$ \\
BD & 1 & $2.658,8203$ & $2.658,8203$ & $93,69 * *$ \\
BE & 1 & $1.946,4641$ & $1.946,4641$ & $68,59 * *$ \\
BF & 1 & $2.334,0977$ & $2.334,0977$ & $82,24 * *$ \\
CD & 1 & $3.085,9414$ & $3.085,9414$ & $108,74 * *$ \\
CE & 1 & $2.596,5394$ & $2.596,5394$ & $91,49 * *$ \\
CF & 1 & $1.543,3112$ & $1.543,3112$ & $54,38 * *$ \\
DE & 1 & $1.898,5628$ & $1.898,5628$ & $66,90 * *$ \\
DF & 1 & $1.630,0397$ & $1.630,0397$ & $57,44 * *$ \\
EF & 1 & $1.252,7175$ & $1.252,7175$ & $44,14 * *$ \\
Residuo & 39 & $1.106,8141$ & 28,379849 & - \\
\hline Tota1 & 63 & $492.443,25$ & & -19 & - \\
\hline
\end{tabular}


Os valores de $F$ por interpolação harmōnica se rão:

$$
\begin{aligned}
& F_{5 \%}(1,39)=4,09 \\
& F_{1 \%}(1,39)=7,33
\end{aligned}
$$

Devemos notar que:

a. Os efeitos principais e as interações de 2 fatores continuam sendo altamente significativos.

$$
\text { b. } 0 \text { QM do residuo confundindo essas } 2 \text { intera }
$$
çoes fica maior do que $O Q M$ do residuo sem confundimento, isto se deve a escolha das interações, pois as suas QM's são pequenas. Se fossem escolhidas as interações $A B E F$ e $A B C$ para serem confundidas, $O Q M$ do residuo diminuiria.

$$
\begin{gathered}
c \cdot \text { A efiència serä: } \\
\widehat{E}=\frac{n_{b} Q M_{B 10 c o s}+n_{e} Q M_{\text {erro }}}{\left(n_{b}+n_{e}\right) Q_{\text {erro }}} \text {, onde } n_{b}=3 \text { e } n_{e}=63-3=60 \\
\widehat{E}=\frac{3 \times 2,5104198+60 \times 28,379849}{63 \times 28,379849}=\frac{1.710,3222}{1.787,9305} \Rightarrow \hat{E}=0,957
\end{gathered}
$$

Caso. fiossem escolhidas as interações $A B E F$ e $A B C$ para serem confundidas com efeito de blocos, teriamos: 


$$
\begin{aligned}
\mathrm{SQ}_{\mathrm{B} 1 \text { ocos }} & =\mathrm{SQ}_{\mathrm{ABEF}}+\mathrm{SQ}_{\mathrm{ABC}}+\mathrm{SQ}_{\mathrm{CEF}} \rightarrow \mathrm{SQ}_{\mathrm{B} 1 \text { ocos }}:= \\
& =356,36983, \mathrm{e} \\
\mathrm{SQ}_{\text {Res }} & =757,97548
\end{aligned}
$$

ou

$$
\begin{aligned}
& \mathrm{QM}_{\mathrm{B} 1 \text { ocos }}=118,78994, \mathrm{e} \\
& \mathrm{QM}_{\text {Res }}=19,435269
\end{aligned}
$$

A eficiēncia seria

$$
\widehat{E}=\frac{3 \times 118,78994+60 \times 19,435269}{63 \times 19,435269}=\frac{1.522,486}{1.224,4219} \Rightarrow \mathrm{E}:=1,243
$$

Comparando estas eficiēncias, vemos que seria melhor escolhermos as interações $A B E F$ e $A B C$ para serem confundidas, pois as mesmas dão uma eficiência maior do que 1 .

Agora vamos trabalhar com $1 / 2$ da repetição do experimento 1 .

Para isto vamos tomar a relação de identidade $I=+A B C D E F$, e assim temos as seguintes combinações de trata mentos, ver exemp1o 1 no item 3.1 .5 .1 .1 . 


\begin{tabular}{|c|c|c|c|}
\hline$(1)=227,46$ & $a e=258,295$ & $a f=258,86$ & ef $=245,21$ \\
\hline$a b=289,07$ & $b e=241,01$ & $b f=240,335$ & abef $=387,15$ \\
\hline$a c=272,71$ & $c e=247,76$ & $\mathrm{cf}=239,65$ & acef $=384,96$ \\
\hline$b c=260,935$ & abce $=428,765$ & $a b c f=422,48$ & bcef $=367,06$ \\
\hline $\mathrm{ad}=271,8$ & $\mathrm{de}=246,955$ & $\mathrm{df}=254,925$ & adef $=375,74$ \\
\hline$b d=250,545$ & abde $=409,915$ & abdf $=410,65$ & bdef $=356,91$ \\
\hline$c d=250,255$ & acde $=397,635$ & $\operatorname{acdf}=389,92$ & cdef $=346,185$ \\
\hline abcd $=437,17$ & bcde $=381,92$ & bcdf $=380,79$ & abcdef $=631,305$ \\
\hline
\end{tabular}

COCHRAN e COX (1957), fazem uma adaptação: do método de Yates para o cálculo das SQ's dos efeitos principais e das interaçoes para experimentos fatoriais fracionados. Assim esta adaptaça consiste em retirar um fator no caso de $1 / 2$ da repeticão, retirar 2 fatores no caso de $1 / 4$ da repeticão, e assim por diante, sendo ignorado temporariamente. Com a retirada dos fatores, o fatorial se torna completo, e assim pode-se aplicar o método de Yates. Feito isso, são reintroduzidos os fatores e assim podemos escrever os conjuntos de aliases com as devidas identificacooes.

No nosso caso, o experimento tem 32 tratamen tos, e se ignorarmos o fator $F$, vamos ter uma repeticão completa dos 5 fatores A, B, C, D e E. Arranjamos esses 5 fato res na "ordem padrão", necessāria para a aplicação do método de Yates, e procedemos como se o experimento fosse um fatorial $2^{5}$ completo. 
Com o fator F ausente, vamos ter na coluna (5) do nosso quadro de cálculos, os totais dos efeitos principais e suas interações desses 5 fatores.

Feito isto, reintroduzimos o fator $F$, com isso, cada efeito passa a ter uma alias envolvendo F, isto ë, $A=B C D E F, B=A C D E F, C=A B D E F$, e assim por diante, os efeitos envolvendo $F$ que vão ser estimados são: o efeito principal F, e as interações AF, BF, CF, DF e EF, cujas alia ses são $A B C D E, B C D E$, $A C D E, A B D E, A B C E$ e $A B C D$, respectivameñ te.

Na coluna (6) do quadro está feita a identif caçao atribuida a cada um dos 32 efeitos fatoriais totais. Aqueles efeitos que aparecem como sinal (+) são atribuidos ao residuo. Os totais nos rodapés do Quadro 2 (anexo 1) são usados para conferir os cälculos.

Assim, o quadro da anảlise de variancia é: 

C. Variação
G . L .
S . Q •
$\mathrm{Q} \cdot \mathrm{M}$.
F

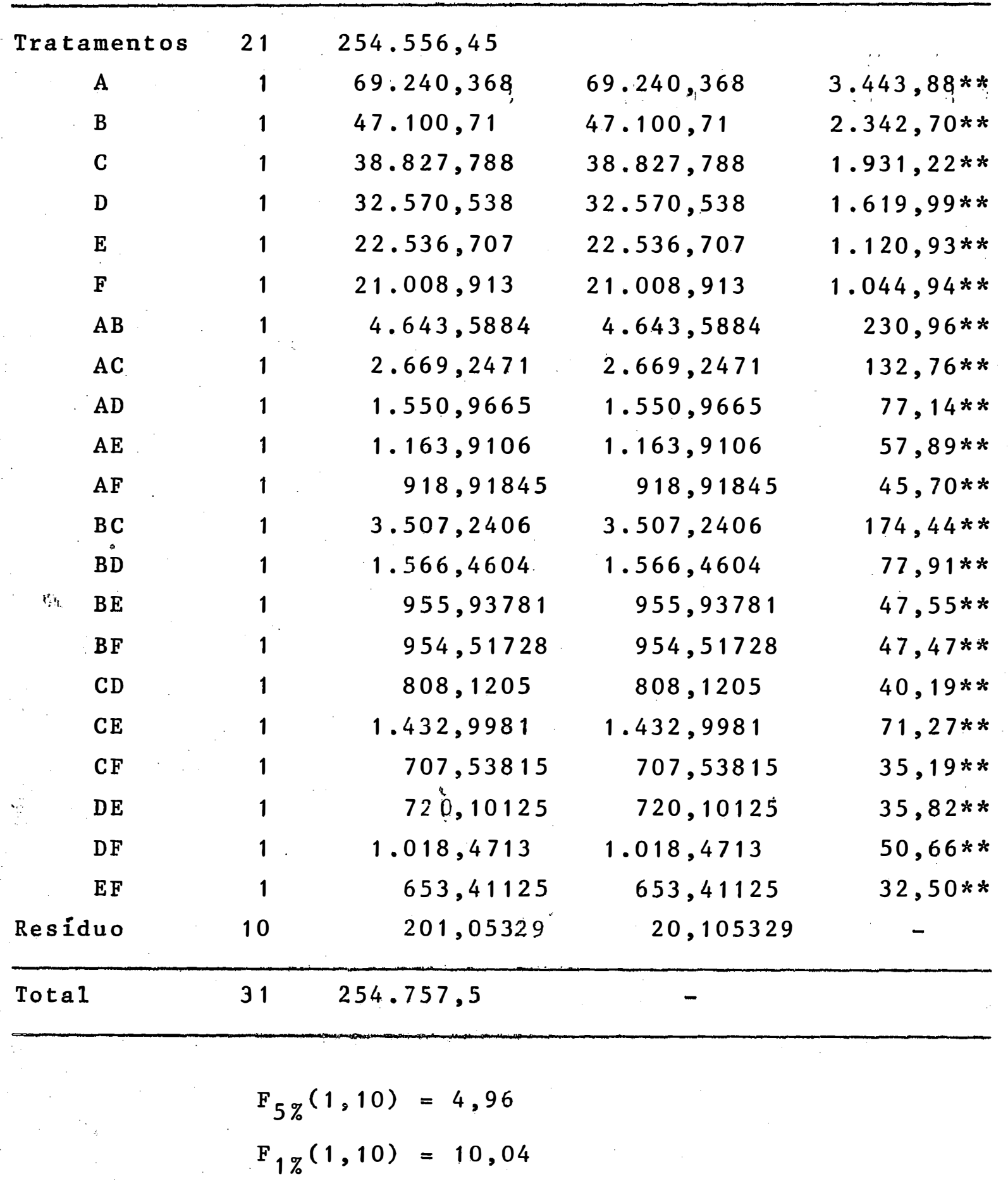


Com $1 / 2$ da repetição, notamos que:

a. Os efeitos principais e as interações de 2 fatores continuam sendo significativos aos niveis de $5 \%$ e $1 \%$.

b. 0 seu $Q M$ do resíduo diminuiu em relação ao QM do residuo do fatorial completo com. e sem confundimento.

$$
\begin{aligned}
& \text { c. A eficiência serä: } \\
& \widehat{\mathrm{E}}=\frac{\mathrm{QM}_{\text {Res. Compl. }}}{\mathrm{QM}_{\text {Res. Frac. }}}=\frac{26,532031}{20,105329} \rightarrow \mathrm{E}=1,320
\end{aligned}
$$

Vemos que a eficiência do fatorial fracionado em relação ao fatorial completo é maior do que 1 .

Vamos agora colocar $1 \% 2$ do fatorial $2^{6}$ em 2 blocos, para isto precisamos confundir uma interação de 3 fa tores, e assim perdemos 1.g.1. do residuo.

A interaça escolhida com sua respectiva alias sera $B C D=A E F$, então temos os seguintes blocos: 
BLOCO I

$$
x_{2}+x_{3}+x_{4}=0(\bmod \cdot 2)
$$

$$
\text { (1) }=227,46 \quad \text { af }=258,86
$$$$
b c=260,935 \quad \text { abcf }=422,48
$$$$
\text { bd }=250,545 \quad \text { abdf }=410,65
$$$$
c d=250,255 \quad \text { acdf }=389,92
$$$$
\mathrm{ae}=258,295
$$

$$
\text { ef }=245,21
$$

abce $=428,765 \quad$ bcef $=367,06$

abde $=409,915 \quad$ bdef $=356,91$

acde $=397,635 \quad$ cdef $=346,185$
BLOCO I I

$$
x_{2}+x_{3}+x_{4}=1(\bmod \cdot 2)
$$

$$
\begin{array}{rlrl}
a b & =289,07 & \text { bf } & =240,335 \\
a c & =272,71 & \text { cf } & =239,65 \\
\text { ad } & =271,8 & \text { df } & =254,925 \\
\text { abcd } & =437,17 & \text { bcdf } & =380,79 \\
\text { be } & =241,01 & \text { abef } & =387,15 \\
c e & =247,76 & \text { acef } & =384,96 \\
\text { de } & =246,955 & \text { adef } & =375,74 \\
\text { bcde } & =381,92 \text { abcdef } & =631,305
\end{array}
$$

$$
5.281,08
$$

Sabemos que a $S Q_{B 1 \text { ocos }}=S Q_{B C D}=\dot{0}_{0,1471531}$ pois a interação BCD estä confundida com blocos, e a $S Q_{\text {Res }}$ c/frac. e conf. $=S_{\text {Res. c/frac. e s/conf. }}-S_{B C D}, \therefore$ como tambēm se perde $1 \mathrm{~g} .1$. do residuo. 0 quadro da anälise de variância serà : 


\section{Variação \\ G.L . \\ S.Q. \\ Q.M . \\ F}

\begin{tabular}{|c|c|c|c|c|}
\hline B1ocos & 1 & 0,1471531 & - & - \\
\hline Tratamentos & 21 & $254.556,45$ & - & - \\
\hline A & 1 & $69.240,368$ & $69.240,368$ & $3.022,84 * *$ \\
\hline B & 1 & $47.100,71$ & $47.100,71$ & $2.056,28 * *$ \\
\hline C & 1 & $38.827,788$ & $38.827,788$ & $1.695,11 * *$ \\
\hline D & 1 & $32.570,538$ & $32.570,538$ & $1.421,94 * *$ \\
\hline $\mathbf{E}$ & 1 & $22.536,707$ & $22.536,707$ & $983,89 * *$ \\
\hline $\mathrm{AB}$ & 1 & $21.008,913$ & $21.008,913$ & $917,19 * *$ \\
\hline $\mathrm{AC}$ & 1 & $4.643,5884$ & $4.643,5884$ & $202,72 * *$ \\
\hline$A D$ & 1 & $2.669 ; 2471$ & $2.669,2471$ & $116,53 * *$ \\
\hline $\mathrm{AE}$ & 1 & $1.550,9665$ & $1.550,9665$ & $67,71 * *$ \\
\hline $\mathrm{AF}$ & 1 & $1.163,9106$ & $1.163,9106$ & $50,81 * *$ \\
\hline BC & 1 & 918,91845 & 918,91845 & $40,12 * *$ \\
\hline BD & $i$ & $3.507,2406$ & $3.507,2406$ & $153,12 * *$ \\
\hline $\mathrm{BE}$ & 1 & $1.566,4604$ & $1.566,4604$ & $68,39 * *$ \\
\hline BF & 1 & 955,51728 & 955,51728 & $41,72 * *$ \\
\hline$C D$ & 1 & 808,1205 & 808,1205 & $35,28 * *$ \\
\hline $\mathrm{CE}$ & 1 & $1.432,9981$ & $1.432,9981$ & $62,56 * *$ \\
\hline$C F$ & 1 & 707,53815 & $707,538.15$ & $30,89 * *$ \\
\hline $\mathrm{DE}$ & 1 & 720,10125 & 720,10125 & $31,44 * *$ \\
\hline $\mathrm{DF}$ & 1 & $1.018,4713$ & $1.018,4713$ & $44,46 * *$ \\
\hline $\mathrm{EF}$ & 1 & $653,41.125$ & 653,41125 & $28,53 * *$ \\
\hline Residuo & 9 & 200,90576 & 22,90576 & - \\
\hline Total & 31 & $254.757,5$ & - & \\
\hline
\end{tabular}


Notamos que:

a. Os efeitos principais e as interações de dois fatores continuam sendo altamente significativos.

b. O QM do resíduo do fatorial fracionado com confundimento em relação a fatorial fracionado sem confundi mento aumentou, isto se deve a interação escolhida.

$$
\begin{gathered}
\text { c. A eficiência serä: } \\
\widehat{\mathrm{E}}=\frac{\mathrm{QM}_{\text {Res.frac. } / \text { fonf. }}}{\mathrm{QM}_{\text {Res.frac. c/conf. }}}=\frac{20,105329}{22,90576} \Rightarrow \widehat{\mathrm{E}}=0,878
\end{gathered}
$$

d. Caso fosse escolhida a interação $A B C$ (ver anexo 1. - Quadro 2) para ser confundida, O QM do residuo diminuiria, ou seja:

$$
\begin{aligned}
& \mathrm{SQ}_{\mathrm{B} 1 \text { ocos }}=\mathrm{SQ}_{\mathrm{ABC}}=78,75125, \mathrm{e} \\
& \mathrm{SQ}_{\text {Res. frac. } \mathrm{c} / \mathrm{conf} .}=122,30204, \text { então: } \\
& \mathrm{QM}_{\text {Res.frac. } \mathrm{c} / \mathrm{conf} .}=\frac{122,30204}{9}=13,589116
\end{aligned}
$$

Assim, a eficiência neste caso serä:

$$
\overline{\mathrm{E}}=\frac{\mathrm{QM}_{\text {Res.frac. } \mathrm{s} / \mathrm{conf} .}}{\mathrm{QM}_{\text {Res. frac. } \mathrm{c} / \mathrm{conf} .}}=\frac{20,105329}{13,589116} \rightarrow \hat{\mathrm{E}}=1,479
$$


Comparando estas eficiências, vemos que seria melhor escolher a interação $A B C$ para ser confundida, pois ela nos dä uma eficiência maior do que 1 .

Agora vamos resumir o experimento 1, montando o quadro abaixo, onde aparece os QM's residuais e as eficiên cias de todos casos vistos:

1. Fatorial completo s/conf.

2. Fatorial completo conf. a int. $A B C D E F$

3. Fatorial completo conf. a int. ABEF

4. Fatorial completo conf, as int's. ABDE e ACDF

5. Fatorial completo conf. as int's. $A B E F$ e $A B C$

6. Fatorial fracionado: $1 / 2$ da rep. s/conf.

7. Fatorial fracionado: $1 / 2$ da rep. conf. int. BCD $: 22,90576$

8. Fatorial fracionado: $1 / 2$ da rep. conf. int. $A B C$
26,532031

$25,787007 \quad 1,019$

$21,433199 \quad 1,159$

$28,379849 \quad 0,957$

$19,435269 \quad 1,243$

$20,105329 \quad 1,320$

$0,878 *$

$1,479 *$

(*) - Estas eficiēncias são calculadas sobre $1 / 2$ da repetição. 


\subsection{EXPER IMENTO 2}

A partir do Quadro 3 (ver anexo 1), obteve-se - seguinte quadro da anälise de variāncia para o fatorial completo:
C. Variação
G.L.
S.Q.
$\mathrm{Q} \cdot \mathrm{M}$.
F

\begin{tabular}{|c|c|c|c|c|}
\hline Tratamentos & 21 & $493.982,22$ & - & - \\
\hline $\mathrm{A}$ & 1 & $136,639,27$ & $136.639,27$ & $1.287,56 * *$ \\
\hline B & 1 & $93.946,848$ & $93.946,848$ & $885,26 * *$ \\
\hline C & 1 & $76.459,163$ & $76.459,163$ & $720,48 * *$ \\
\hline $\mathrm{D}$ & 1 & $59.971,112$ & $59.971,112$ & $565,11 * *$ \\
\hline $\mathbf{E}$ & 1 & $40.616,354$ & $40.616,354$ & $382,73 * *$ \\
\hline $\mathbf{F}$ & 1 & $38.652,543$ & $38.652,543$ & $364,22 * *$ \\
\hline $\mathrm{AB}$ & 1 & $8.944,9035$ & $8.944,9035$ & $84,29 * *$ \\
\hline $\mathrm{AC}$ & 1 & $5.009,4545$ & $5.009,4545$ & $47,20 * *$ \\
\hline $\mathrm{AD}$ & 1 & $3.560,5089$ & $3.560,5089$ & $33,55 * *$ \\
\hline $\mathrm{AE}$ & 1 & $2.308,8025$ & $2.308,8025$ & $21,76 * *$ \\
\hline $\mathrm{AF}$ & 1 & $1.943,7077$ & $1.943,7077$ & $18,32 * *$ \\
\hline B C & 1 & $6.766,2963$ & $6.766,2963$. & $63,76 * *$ \\
\hline $\mathrm{BD}$ & 1 & $2.221,7082$ & $2.221,7082$ & $20,94 * *$ \\
\hline $\mathrm{BE}$ & 1 & $1.783,3729$ & $1.783,3729$ & $16,80 * *$ \\
\hline B F & 1 & $3.205,5413$ & $3.205,5413$ & $30,21 * *$ \\
\hline CD & 1 & $3.035,459$ & $3.035,459$ & $28,60 * *$ \\
\hline $\mathrm{CE}$ & 1 & $3.127,0464$ & $3.127,0464$ & $29,47 * *$ \\
\hline $\mathrm{CF}$ & 1 & $1.488,2235$ & $1.488,2235$ & $14,02 * *$ \\
\hline $\mathrm{DE}$ & 1 & $1.693,5283$ & $1.693,5283$ & $15,96 * *$ \\
\hline $\mathrm{DF}$ & 1 & $1.660,97$ & $1.660,97$ & $15,65 * *$ \\
\hline $\mathrm{EF}$ & 1 & 947,4084 & 947,4084 & $8,93 * *$ \\
\hline Residuo & 42 & $4.457,1672$ & 106,12303 & - \\
\hline a 1 & 63 & $498.439,39$ & - & \\
\hline
\end{tabular}


Os valores de F são os mesmos do experimento 1 .

Assim, vemos que os efeitos principais: e as interaçoes de 2 fatores continuam sendo significantes aos ni veis de $5 \%$ e $1 \%$.

Agora vamos confundir a interação de ordem mais elevada, ou seja, ABCDEF. Para formar os 2 blocos procedemos da mesma maneira como no experimento 1.

Como a interação $A B C D E F$ está confundida, então a $\mathrm{SQ}_{\mathrm{B} 1 \text { ocos }}=\mathrm{SQ}_{\mathrm{ABCDEF}}=228,08551$, e a $\mathrm{SQ}_{\mathrm{Res}} \mathrm{c} / \mathrm{conf}$. $\mathrm{SQ}_{\text {Res. s/conf. }} \mathrm{SQ}_{\mathrm{ABCDEF}}$, como também se perde $1 \mathrm{~g} .1$. do re siduo. o quadro da análise de variância serä: 


\begin{tabular}{|c|c|c|c|c|}
\hline C. variação & G.L. & $S \cdot Q=$ & $Q \cdot M$. & $\mathrm{F}$ \\
\hline B1ocos & $\cdot 1$ & 228,08551 & - & - \\
\hline Tratamentos & 21 & $493.982,22$ & - & - \\
\hline A & 1 & $136.639,27$ & $136.639,27$ & $1.324,69 * *$ \\
\hline B & 1 & $93.946,848$ & $93.946,848$ & $910,79 * *$ \\
\hline C & 1 & $76.459,163$ & $76.459,163$ & $741,25 * *$ \\
\hline D & 1 & $59.971,112$ & $59.971,112$ & $581,41 * *$ \\
\hline E & 1 & $40.616,356$ & $40.616,356$ & $393,77 * *$ \\
\hline F & 1 & $38.652,543$ & $38.652,543$ & $374,73 * *$ \\
\hline $\mathrm{AB}$ & 1 & $8.944,9035$ & $8.9,44,9035$ & $86,72 * *$ \\
\hline $\mathrm{AC}$ & 1 & $5.009,4545$ & $5.009,4545$ & $48,56 * *$ \\
\hline $\mathrm{AD}$ & 1 & $3.560,5089$ & $3.560,5089$ & $34,52 * *$ \\
\hline $\mathrm{AE}$ & 1 & $2.308,8025$ & $2.308,8025$ & $22,38 * *$ \\
\hline $\mathrm{AF}$ & 1 & $1.943,7077$ & $1.943,7077$ & $18,84 * *$ \\
\hline $\mathrm{BC}$ & 1 & $6.766,2963$ & $6.766,2963$ & $65,60 * *$ \\
\hline $\mathrm{BD}$ & 1 & $2.221,7082$ & $2.221,7082$ & $21,54 * *$ \\
\hline $\mathrm{BE}$ & 1 & $1.783,3729$ & $1.783,3729$ & $17,29 * *$ \\
\hline BF & 1 & $3.205,5413$ & $3.205,5413$ & $31,08 * *$ \\
\hline$C D$ & 1 & $3.035,459$ & $3.035,459$ & $29,43 * *$ \\
\hline $\mathrm{CE}$ & 1 & $3.127,0464$ & $3.127,0464$ & $30,32 * *$ \\
\hline$C F$ & 1 & $1.488,2235$ & $1.488,223.5$ & $14,43 * *$ \\
\hline $\mathrm{DE}$ & 1 & $1.693,5283$ & $1.693,5283$ & $16,42 * *$ \\
\hline $\mathrm{DF}$ & 1 & $1.660,97$ & $1.660,97$ & $16,10 * *$ \\
\hline $\mathrm{EF}$ & 1 & 947,4084 & 947,4084 & $9,18 * *$ \\
\hline Residuo & 41 & $4.229,0817$ & 103,14833 & - \\
\hline that & S & 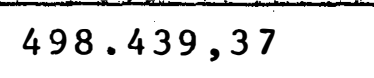 & & \\
\hline
\end{tabular}

os valores de $F$ são os mesmos do experimento 1 
Podemos observar que:

a. Os efeitos principais e as interações de 2 fatores continuam sendo significativos.

b. 0 QM do residuo com confundimento fica menor do que $O$ QM do residuo sem confundimento e esta diferença è de 2,9747.

c. Esta diferença entre os QM's serà maior ou menor devido a escolha da interação a ser confundida, por exemplo, se escolhessemos a interação $A B E F$, $O$ QM do residuo seria 85,738807. Mas, se fosse escolhida a interação ACDF, o QM do residuo seria 108,69656 , como vemos o mesmo aumentou em relação ao QM do residuo do fatorial completo.

$$
\begin{aligned}
& \text { d. A eficiência serả: . }
\end{aligned}
$$

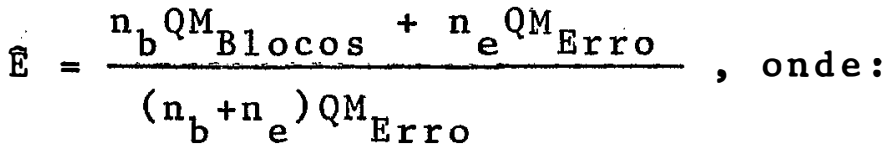

$$
\begin{aligned}
& \mathrm{n}_{\mathrm{b}}=1 \\
& \mathrm{n}_{e}=63-1=62 \text {, então: } \\
& \widehat{\mathrm{E}}=\frac{1 \times 228,08551+62 \times 103,14833}{63 \times 103,14833}=\frac{6.623,282}{6.498,3448} \rightarrow \widehat{\mathrm{E}}=1,019
\end{aligned}
$$


e. Se a interação escolhida fosse ABEF,

eficiência seria:

$$
\begin{gathered}
\mathrm{QM}_{\mathrm{B} 1 \text { ocos }}=941,8761 \\
Q M_{\text {Erro }}=\frac{3.515,2911}{41}=85,738807 \\
\hat{\mathrm{E}}=\frac{1 \times 941,8761+62 \times 85,738807}{63 \times 85,738807}=\frac{6257,6821}{5401,5448} \rightarrow \hat{\mathrm{E}}=1,158
\end{gathered}
$$

Como foi visto para o experimento 1, a eficiência da interação $A B E F$ quando confundida, continua sendo maior do que a interação ABCDEF.

Agora vamos confundir as interações $A B D E$ e ACDF, como sabemos a interação generalizada BCEF também estạ rà confundida.

Para formar os 4 blocos procedemos da mesma maneira como no experimento 1.

$$
\text { Como as interações ABDE, ACDF e BCEF estão }
$$

confundidas, então a

$$
\begin{aligned}
& \mathrm{SQ}_{\mathrm{B} 1 \mathrm{ocos}}=\mathrm{SQ}_{\mathrm{ABDE}}+\mathrm{SQ}_{\mathrm{ACDF}}+\mathrm{SQ}_{\mathrm{BCEF}}=11,172306+0,6084+ \\
& +18,3184 \rightarrow \\
& S Q_{B 10 c o s}=30,099106 \text {, então: } \\
& \mathrm{SQ}_{\text {Res. c/conf. }}=\mathrm{SQ}_{\mathrm{Res} . \mathrm{s} / \mathrm{conf} .}-\mathrm{SQ}_{\mathrm{B} 1 \mathrm{ocos}}=4.457,1672 \\
& -30,099106 \rightarrow
\end{aligned}
$$


$\mathrm{SQ}_{\text {Res. c/conf. }}=4.427,0681$

Assim, temos o quadro da anālise de variância:
C. Variância
G.L.
S.Q.
Q.M.
F

\begin{tabular}{|c|c|c|c|c|}
\hline B1ocos & 3 & 30,099106 & 10,03303 & \\
\hline Tratamentos & 21 & $493.982,22$ & - & \\
\hline A & 1 & $136.639,27$ & $136.639,27$ & $1.206,90 * *$ \\
\hline B. & 1 & $93.946,848$ & $93.946,848$ & $827,62 * *$ \\
\hline C & 1 & $76.459,163$ & $76.459,163$ & $673,56 * *$ \\
\hline D & 1 & $59.97 .1,112$ & $59.971,112$ & $528,31 * *$ \\
\hline E & 1 & $40.616,356$ & $40.616,356$ & $357,81 * *$ \\
\hline F & 1 & $38.652,543$ & $38.652,543$ & $340,51 * *$ \\
\hline$A B$ & 1 & $8.944,9035$ & $8.944,9035$ & $78,80 * *$ \\
\hline $\mathrm{AC}$ & 1 & $5.009,4545$ & $5.009,4545$ & $44,13 * *$ \\
\hline $\mathrm{AD}$ & 1 & $3.560,5089$ & $3.560,5089$ & $31,37 * *$ \\
\hline $\mathrm{AE}$ & 1 & $2.308,8025$ & $2.308,8025$ & $20,34 * *$ \\
\hline $\mathrm{AF}$ & 1 & $1.943,7077$ & $1.943,7077$ & $17,12 * *$ \\
\hline $\mathrm{BC}$ & 1 & $6.766,2963$ & $6.766,2963$ & $59,61 * *$ \\
\hline BD & 1 & $2.221,7082$ & $2.221,7082$ & $19,57 * *$ \\
\hline BE & 1 & $1.783,3729$ & $1.783,3729$ & $15,71 * *$ \\
\hline BF & 1 & $3.205,5413$ & $3.2 .05,5413$ & $28,24 * *$ \\
\hline CD & 1 & $3.035,459$ & $3.035,459$ & $26,74 * *$ \\
\hline $\mathrm{CE}$ & 1 & $3.127,0464$ & $3.127,0464$ & $27,55 * *$ \\
\hline $\mathrm{CF}$ & 1 & $1.488,2235$ & $1.488,2235$ & $13,11 * *$ \\
\hline $\mathrm{DE}$ & 1 & $1.693,5283$ & $1.693,5283$ & $14,92 * *$ \\
\hline $\mathrm{DF}$ & 1 & $1.660,97$ & $1.660,97$ & $14,63 * *$ \\
\hline EF & 1 & 947,4084 & 947,4084 & $8,35 * *$ \\
\hline Residuo & 39 & $4.427,0681$ & 113,51457 & - \\
\hline Total & 63 & $498.439,37$ & - & - \\
\hline
\end{tabular}

Os valores de $F$ são os mesmos do experimento 1 . 


\section{Devemos notar que:}

a. Os efeitos principais e as interações de 2 fatores continuam sendo significativos.

b. 0 QM do resíduo confundindo essas 2 intera ções fica maior do que o QM do residuo sem confundimento, is to se deve a escolha das interações pois as suas SQ's. são pequenas. Se fossem escolhidas as interações $A B E F$ e $A B C$ para serem confundidas, o QM do residuo diminuiria.

c. A eficiência serà:

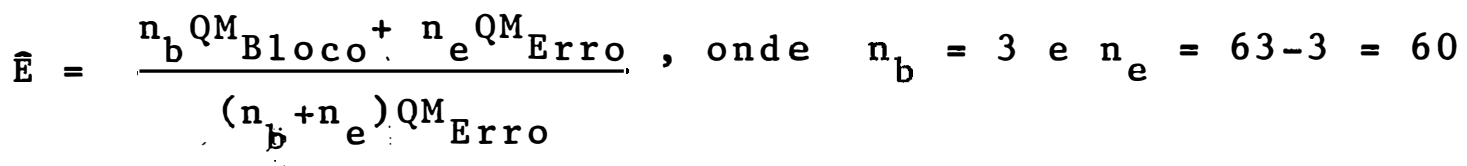

$$
\begin{aligned}
& \hat{\mathrm{E}}=\frac{3 \times 10,033035+60 \times 113,51457}{63 \times 113,51457}=\frac{6.840,9733}{7.151,4179}-\hat{\mathrm{E}}=0,957
\end{aligned}
$$

Caso fossem escolhidas as interações ABEF e ABC para serem confundidas com efeitos de blocos, terímos:

$$
\begin{aligned}
& S_{B 1 \text { ocos }}=S Q_{A B E F}+S Q_{A B C}+S Q_{C E F} \rightarrow Q_{B 1 o c o s}=1.435,4173 \\
& S Q_{\text {Res }}=3.021,7499
\end{aligned}
$$

ou

$$
\mathrm{QM}_{\mathrm{B} 1 \text { ocos }}=478,47243
$$




$$
\begin{aligned}
& { }_{\text {Res }}=77,480767 \\
& \text { A eficiência seria: } \\
& \vec{E}=\frac{3 \times 478,47243+60 \times 77,480767}{63 \times 77,480767}=\frac{6.084,2633}{4.881,2883} \rightarrow E=1,246
\end{aligned}
$$

Comparando estas eficiências, vemos que seria melhor escolhermos as interações ABEF e ABC pará serem confundidas, pois as mesmas dão uma eficiência maior do que 1.

Agora vamos trabalhar com $1 / 2$ da repetição do experimento 2 .

Para isto vamos tomar a relação de identidade $I=+A B C D E F, e$ assim temos as seguintes combinações de tratamentos, ver exemplo 1, do item 3.1.5.1.1.

$$
\begin{aligned}
& (1)=226,02 \text { ae }=253,09 \quad \text { af }=256,22 \text { ef }=242,52 \\
& a b=290,04 \quad b e=232,52 \quad b f=233,17 \text { abef }=381,20 \\
& \text { ac }=271,42 \quad \text { ce }=247,92 \quad \text { cf }=233,70 \text { acef }=390,92 \\
& \mathrm{bc}=261,87 \quad \mathrm{abce}=433,13 \quad \mathrm{abcf}=428,56 \quad \mathrm{bcef}=369,12 \\
& \mathrm{ad}=276,60 \quad \mathrm{de}=245,51 \quad \mathrm{df}=263,45 \text { adef }=379,48 \\
& b d=248,09 \quad a b d e=410,23 \quad a b d f=419,70 \quad b d e f=355,82 \\
& \text { cd }=249,41 \text { acde }=399,77 \text { acdf }=392,34 \text { cdef }=336,27 \\
& \text { abcd }=436,44 \quad \text { bcde }=382,34 \quad \text { bcdf }=388,08 \text { abedef }=633,71
\end{aligned}
$$


pregado para o experimento 1, assim todas as observações dada anteriormente são vālidas.

No Quadro 4 (anexo 1), temos o quadro com a adaptaçào do procedimento de Yates. Assim o quadro da anāli se de variāncia é:

\begin{tabular}{crccc}
\hline C: Variaço & G.L. & S.Q. & Q.M. & F \\
\hline Tratamentos & 21 & $265.478,64$ & - & - \\
A & 1 & $73.827,874$ & $73.827,874$ & $920,12 * *$ \\
B & 1 & $48.001,962$ & $48.001,962$ & $598,25 * *$ \\
C & 1 & $40.708,031$ & $40.708,031$ & $507,35 * *$ \\
D & 1 & $35.499,133$ & $35.499,133$ & $442,43 * *$ \\
E & 1 & $20.932,626$ & $20.932,626$ & $260,88 * *$ \\
F & 1 & $22.042,651$ & $22.042,651$ & $274,72 * *$ \\
AB & 1 & $4.679,3138$ & $4.679,3138$ & $58,32 * *$ \\
AC & 1 & $2.777,3605$ & $2.777,3605$ & $34,61 * *$ \\
AD & 1 & $1.534,0261$ & $1.534,0261$ & $19,12 * *$ \\
AE & 1 & $1.274,8725$ & $1.274,8725$ & $15,89 * *$ \\
AF & 1 & $1.046,0738$ & $1.046,0738$ & $13,04 * *$ \\
BC & 1 & $4.598,8845$ & $4.598,8845$ & $57,32 * *$ \\
BD & 1 & $1.564,9215$ & $1.564,9215$ & $19,50 * *$ \\
BE & 1 & 859,05125 & 859,05125 & $10,71 * *$ \\
BF & 1 & $1.122,6691$ & $1.122,6691$ & $13,99 * *$ \\
CD & 1 & 291,73201 & 291,73201 & 3,64 \\
CE & 1 & $1.864,7725$ & $1.864,7725$ & $23,24 * *$ \\
CF & 1 & 620,75261 & 620,75261 & $7,74 *$ \\
DE & 1 & 447,005 & 447,005 & $5,57 *$ \\
DF & 1 & $1.263,2851$ & $1.263,2851$ & $15,74 * *$ \\
EF & 1 & 521,645 & 521,645 & $6,56 *$ \\
Residuo & 10 & 802,37015 & 80,237015 & - \\
\hline Tota1 & 31 & $266.281,01$ & - & - \\
\hline & 1 & & & \\
\hline
\end{tabular}




$$
\begin{aligned}
& F_{5 \%}(1,10)=4,96 \\
& F_{1 \%}(1,10)=10,04
\end{aligned}
$$

Com $1 / 2$ da repetição, notamos que:

a. Os efeitos principais e a maioria das inte raçoes de 2 fatores continuam sendo significativas aos niveis de $5 \%$ e $1 \%$, enquanto que as interações CF, DE e EF são : somente ao nível de $5 \%$ e a interação $C D$ é não significativa.

b. 0 seu QM do residuo diminuiu em relação ao QM do residuo do fatorial completo com e sem confundimento.

c. A eficiência serä:

$$
\widehat{\mathrm{E}}=\frac{\mathrm{QM}_{\text {Res._comp } 1 .}}{\mathrm{QM}_{\text {Res. frac. }}}=\frac{106,12303}{80,237015} \rightarrow \cdot \hat{\mathrm{E}}=1,323
$$

Vemos que a eficiência do fatorial fracionado em relação ao fatorial completo é maior do que 1 .

Vamos agora colocar $1 / 2$ do fatorial $2^{6}$ em 2 blocos, para isto precisamos confundir uma interação de 3 fa tores, e assim perdemos 1 g.1. do residuo.
A interação escolhida com sua respectiva aliäs sera $B C D=A E F$. 
Para formar os 2 blocos procedemos da mesma maneiracomo no experimento 1 .

Sabemos que a $S Q_{B 1 \text { ocos }}=S Q_{B C D}=0,5886125$, e a $S Q_{\text {Res. c/frac. e conf. }}=S Q_{\text {Res. c/frac. s/conf. }} \mathrm{SQ}_{\mathrm{BCD}}$, como tambëm perdemos $1 \mathrm{~g} .1$. do residuo. 0 quadro da anälise de variância serä:

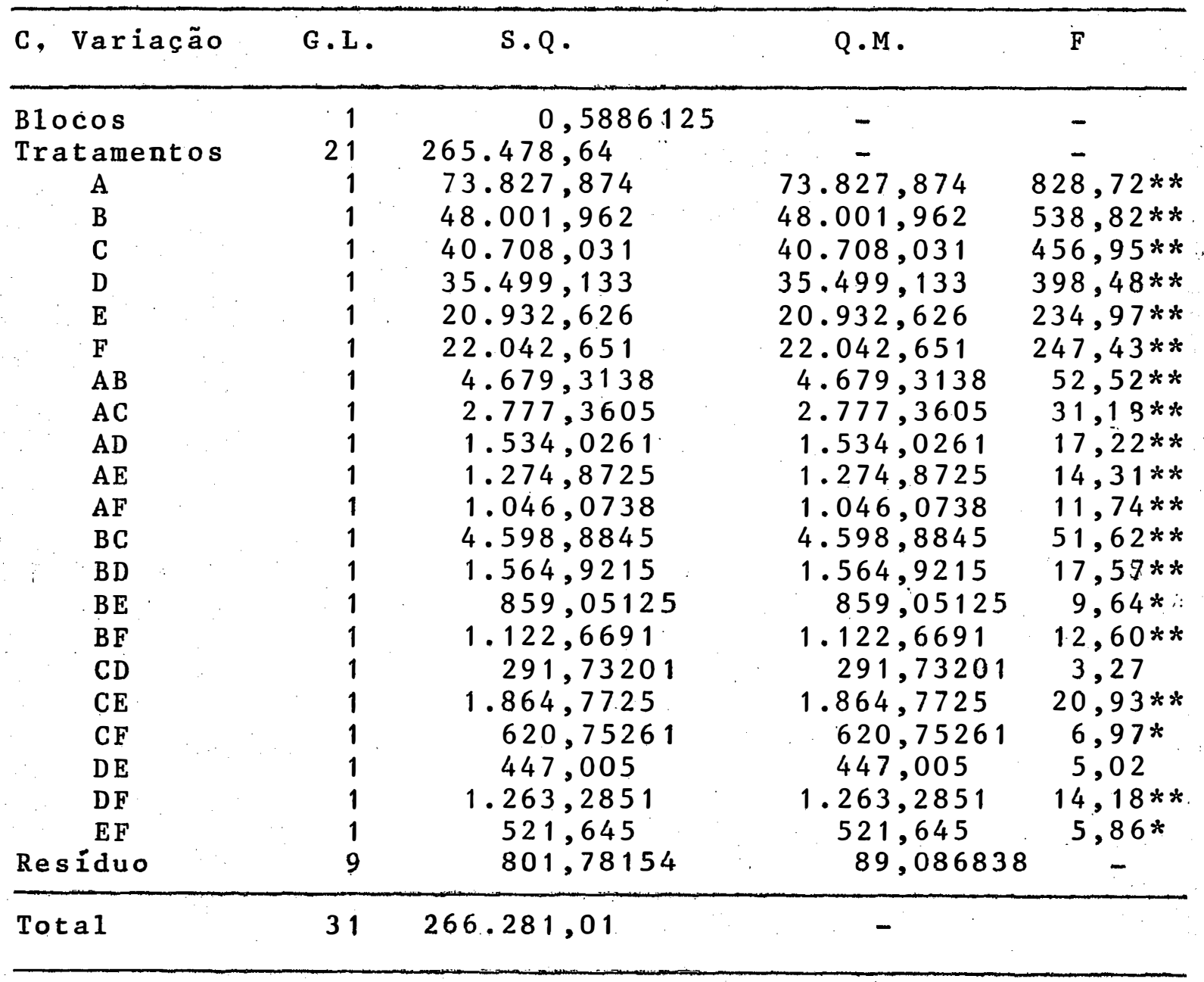

$F_{5 \%}(1,9)=5,12$
$F_{1 \%}(1,9)=10,56$


Notamos que:

a. Os efeitos principais continuam sendo alta mente significativos, e as interações BE, CF e EF são significativas somente a nivel de $5 \%$, enquanto as interacooes CD e DE são não significativas.

b. A interação DE que sem confundimento era significativa ao nível de $5 \%$, aqui ela não é significativa, isto se deve ao aumento do $Q M$ do residuo.

c. Como já sabemos pelo item b, o QM do resí duo do fatorial fracionado com confundimento aumentou em relação ao mesmo seu confundimento, isto se deve a interação escolhida.

$$
\begin{gathered}
\text { d. A eficiência serä: } \\
E=\frac{Q M \text { Res.frac. s/conf. }}{Q M_{\text {Res.frac. c/conf. }}}=\frac{80,237015}{89,086838}=E=0,901
\end{gathered}
$$

e. Caso fosse escolhida a interação $A B C$ (ver anexo 1 - quadro 4) para ser confundida, O QM do resíduo diminuiria, ou seja:

$$
\mathrm{SQ}_{\mathrm{B} 1 \text { ocos }}=\mathrm{SQ}_{\mathrm{ABC}}=315,005, \mathrm{e}
$$


$\mathrm{SQ}_{\text {Res.frac. c/conf. }}=487,36515$, então,

$\mathrm{QM}_{\text {Res. frac. c/conf. }}=\frac{487,36515}{9}=54,151683$

Assim, a eficiēncia neste caso serā:

$\overline{\mathrm{E}}=\frac{\mathrm{QM}_{\mathrm{Res} \cdot \mathrm{frac} \cdot \mathrm{s} / \mathrm{conf}}}{\mathrm{QM}_{\text {Res.frac. c/conf. }}}=\frac{80,237015}{54,151683} \rightarrow \overline{\mathrm{E}}=1,482$

Comparando estas eficiēncias, vemos que seria melhor escolher a interação $A B C$ para ser confundida, pois ela nos dä uma eficiēncia maior do que 1 .

Agora vamos resumir o experimento 2 , montando o quadro abaixo, onde aparecem os QM's residuais e as eficiencias de todos casos vistos:

Experimento 2

$Q \cdot M$.

$\overline{\mathrm{E}}$

1. Fatorial completo s/conf.

106,12303

2. Fatorial completo conf. a int. ABCDEF

$103,148331,019$

3. Fatorial completo conf. a int. ABEF

$85,7388071,158$

4. Fatorial completo conf. as int's ABDE e ACDF

$113,51457 \quad 0,957$

5. Fatorial completo conf. as int's $A B E F$ e $A B C$

$77,4807671,246$

6. Fatorial fracionado: $1 / 2$ da rep. s/conf.

$80,2370151,323$

7. Fatorial fracionado: $1 / 2$ da rep. conf. a int. BCD 89,086838 0,901(*)

8. Fatorial fracionado: $1 / 2$ da rep. conf. a int. ABC $54,1516831,482(*)$

(*) Estas eficiências foram calculadas sobre $1 / 2$ da repetição 


\subsection{EXPER IMENTO 3}

A partir do Quadro 5 (ver anexo 1), obteve-se o seguinte quadro da anälise de variancia para o fatorial completo:

C. Variação G.L. S.Q. Q.M.

\begin{tabular}{|c|c|c|c|c|}
\hline Tratamentos & 21 & $497.150,72$ & - & - \\
\hline $\mathrm{A}$ & 1 & $140.140,26$ & $140.140,26$ & $586 ; 98 * *$ \\
\hline B & 1 & $94.789,71$ & $94.789,71$ & $397,03 * *$ \\
\hline C & 1 & $77.646,171$ & $77.646,171$ & $325,22 * *$ \\
\hline $\mathrm{D}$ & 1 & $60.247,237$ & $60.247,237$ & $252,35 * *$ \\
\hline $\mathrm{E}$ & 1 & $37.025,697$ & $37.025,697$ & $155,08 * *$ \\
\hline$F$ & 1 & $37.941,44$ & $37.941,44$ & $158,92 * *$ \\
\hline $\mathrm{AB}$ & 1 & $8.788,7109$ & $8.788,7109$ & $36,81 * *$ \\
\hline $\mathrm{AC}$ & 1 & $4.968,0471$ & $4.968,0471$ & $20,81 * *$ \\
\hline $\mathrm{AD}$ & 1 & $3.768,3484$ & $3.768,3484$ & $15,78 * *$ \\
\hline $\mathrm{AE}$ & 1 & $2.396,7756$ & $2.396,7756$ & $10,04 * *$ \\
\hline $\mathrm{AF}$ & 1 & $2.139,0047$ & $2.139,0047$ & $8,96 * *$ \\
\hline $\mathrm{BC}$ & 1 & $7.650,6729$ & $7.650,6729$ & $32,04 * *$ \\
\hline $\mathrm{BD}$ & 1 & $1.833,6059$ & $1.833,6059$ & $7,68 * *$ \\
\hline $\mathrm{BE}$ & 1 & $1.637,2645$ & $1.637,2645$ & $6,86 *$ \\
\hline $\mathrm{BF}$ & 1 & $4.200,0931$ & $4.200,0931$ & $15,59 * *$ \\
\hline CD & 1 & $2.972,9075$ & $2.972,9075$ & $12,45 * *$ \\
\hline $\mathrm{CE}$ & 1 & $3.692,0055$ & $3.692,0055$ & $15,46 * *$ \\
\hline $\mathrm{CF}$ & 1 & $1.442,8127$ & $1.442,8127$ & $6,04 *$ \\
\hline $\mathrm{DE}$ & 1 & $1.509,0797$ & $1.509,0797$ & $6,32 *$ \\
\hline $\mathrm{DF}$ & 1 & $1.682,179$ & $1.682,179$ & $7,05 *$ \\
\hline $\mathrm{EF}$ & 1 & 678,70019 & 678,70019 & 2,84 \\
\hline Resíduo & 42 & $10.027,374$ & 238,747 & - \\
\hline Tota 1 & 63 & $504.118,68$ & - & - \\
\hline
\end{tabular}


Os valores de F são os mesmos do experimento

1 .

Vemos que os efeitos principais e a maioria das interaçös de 2 fatores continuam sendo significantes aos niveis de $5 \%$ e $1 \%$, enquanto que a interaçäo EF é não sig nificante.

Agora vamos confundir a interaça de ordem mais elevada, ou seja, ABCDEF. Para formar os 2 blocos procedemos dia mesma maneira como no experimento 1.

Como a interação $A B C D E F$ está confundida, então a $\mathrm{SQ}_{\mathrm{B} 1 \text { ocos }}=\mathrm{SQ}_{\mathrm{ABCDEF}}=518,55829$, e a $\mathrm{SQ}_{\mathrm{Res}}$. c/conf. $=$ $=S Q_{\text {Res s/conf. }}-S_{A B C D E F}$, como também se perde $1 \mathrm{~g} \cdot 1$. do residuo. 0 quadro da anālise de variāncia serā: 


\begin{tabular}{|c|c|c|c|c|c|}
\hline C. & Variaçāo & $\mathrm{G} \cdot \mathrm{L} \cdot$ & $S \cdot Q \cdot$ & $Q \cdot M \cdot$ & $\mathrm{F}$ \\
\hline \multicolumn{2}{|c|}{ B1ocos } & 1 & 518,55829 & - & - \\
\hline \multicolumn{2}{|c|}{ Tratamentos } & 21 & $497.150,72$ & - & - \\
\hline & A & 1 & $140.140,26$ & $140.140,26$ & $604,26 * *$ \\
\hline & B & 1 & $94.789,71$ & $94.789,71$ & $408,71 * *$ \\
\hline & $\mathrm{C}$ & 1 & $77.646,171$ & $77.646,171$ & $334,79 * *$ \\
\hline & $\mathrm{D}$ & 1 & $60.247,237$ & $60.247,237$ & $259,77 * *$ \\
\hline & $\mathrm{E}$ & 1 & $37.025,697$ & $37.025,697$ & $159,65 * *$ \\
\hline & $\mathbf{F}$ & 1 & $37.941,44$ & $37.941,44$ & $163,60 * *$ \\
\hline & $\mathrm{AB}$ & 1 & $8.788,7109$ & $8.788,7109$ & $37,90 * *$ \\
\hline & $\mathrm{AC}$ & 1 & $4.968,0471$ & $4.968,0471$ & $21,21 * *$ \\
\hline & $A D$ & 1 & $3.768,3484$ & $3.768,3484$ & $16,25 * *$ \\
\hline & $\mathrm{AE}$ & 1 & $2.396,7756$ & $2.396,7756$ & $10,33 * *$ \\
\hline & $\mathrm{AF}$ & 1 & $2.139,0047$ & $2.139,0047$ & $9,22 * *$ \\
\hline & B C & 1 & $7.650,6729$ & $7.650,6729$ & $32,99 * *$ \\
\hline & B D & 1 & $1.833,6059$ & $1.833,6059$ & $7,91 * *$ \\
\hline & $\mathrm{BE}$ & 1 & $1.637,2645$ & $1.637,2645$ & $7,06 *$ \\
\hline & BF & 1 & $4.200,0931$ & $4.200,0931$ & $18,11 * *$ \\
\hline & CD & 1 & $2.972,9075$ & $2.972,9075$ & $12,82 * *$ \\
\hline & $\mathrm{CE}$ & 1 & $3.692,0055$ & $3.692,0055$ & $15,92 * *$ \\
\hline \multirow[t]{4}{*}{$i$} & $\mathrm{CF}$ & 1 & $1.442,8127$ & $1.442,8127$ & $6,22 * *$ \\
\hline & $\mathrm{DE}$ & 1 & $1.509,0797$ & $1.509,0797$ & $6,51 *$ \\
\hline & $\mathrm{DF}$ & 1 & $1.682,179$ & $1.682,179$ & $7,25 *$ \\
\hline & $\mathrm{EF}$ & 1 & 678,70019 & 678,70019 & 2,93 \\
\hline \multicolumn{2}{|c|}{ Residuo } & 41 & $9.508,8158$ & 231,92234 & - \\
\hline \multicolumn{2}{|c|}{ Tota 1} & 63 & $504.118,68$ & - & - \\
\hline
\end{tabular}

Os valores de F são os mesmos do experimento 1. 
Podemos observar, que:

a. Confundindo a interação ABCDEF, em termos de significāncia continua a mesma coisa.

b. 0 QM do residuo com confundimento fica menor do que o $Q M$ do residuo sem confundimento, e esta diferen ça è de 6,82466 .

c. Esta diferença entre o QM's serā maior ou menor devido a escolha da interação a ser confundida, por exemp1o, se escolhessemos a interação ABEF, o QM do resíduo seria 193,1467. Mas, se fosse escolhida a interação. ACDF, - QM do resíduo seria 244,52963 , como vemos o mesmo aumentou em relação ao QM do resíduo do fatorial completo.

d. A eficiência serā:

$$
\begin{gathered}
\hat{E}=\frac{n_{b}{ }^{Q M} B 10 \cos +n^{Q M} \operatorname{Erro}}{\left(n_{b}+n_{e}\right) Q M_{E r r o}}, \text { onde: } \\
n_{b}=1 \\
n_{e}=63-1=62, \text { então }
\end{gathered}
$$

$$
E=\frac{1 \times 518,55829+62 \times 231,92234}{63 \times 231,92236}=\frac{14.897,743}{14.611,107} \rightarrow E=1,019
$$


e. Se a interação escolhida para ser confundí da fosse ABEF, a eficiēncia seria:

$$
\begin{gathered}
\mathrm{MM}_{\mathrm{B} 1 \mathrm{ocos}}=2.108,3594 \\
\mathrm{QM}_{\mathrm{Erro}}=\frac{7.919,0146}{41}=193,1467 \\
E=\frac{1 \times 2.108,3594+62 \times 193,1467}{63 \times 193,1467}=\frac{14.083,455}{12.168,242} \rightarrow \quad E=1,157
\end{gathered}
$$

Como foi visto para os experimentos 1 e 2 , a eficiência da interação ABEF quando confundida, continua sen do maior do que a interação $A B C D E F$.

Agora vamos confundir as interações $A B D E$ e ACDF, como sabemos a interação generalizada BCEF também esta rá confundida.

Para formar os 4 blocos procedemos da mesma maneira como no experimento 1 .

Como as interações $A B D E, A C D F$ e BCEF estão confundidas, então a $\mathrm{SQ}_{\mathrm{B} 1 \text { ocos }}:=\mathrm{SQ}_{\mathrm{ABDE}}+\mathrm{SQ}_{\mathrm{ACDF}}+\mathrm{SQ}_{\mathrm{BCEF}}=$ $=26,336141+1,659266+39,713628 \rightarrow \mathrm{SQ}_{\mathrm{B} 1 \text { ocos }}=67,709035$, então:

$$
\begin{aligned}
\mathrm{SQ}_{\text {Res. }} \text { c/conf. }= & \mathrm{SQ}_{\text {Res. s/conf. }}-\mathrm{SQ}_{\mathrm{B} 1 \mathrm{cos}}=10.027,374 \\
& -67,709035 \rightarrow
\end{aligned}
$$


$\mathrm{SQ}_{\text {Res. }} \mathrm{c/conf} .=9.959,665$

Assim, temos o quadro da anälise de variāncia:
C. Variação
G.L .
S.Q.
Q.M.
F

\begin{tabular}{crccc} 
Blocos & 3 & 67,709035 & 22,569678 & - \\
Tratamentos & 21 & $497.150,72$ & - & - \\
A & 1 & $140.140,26$ & $140.140,26$ & $548,76 * *$ \\
B & 1 & $94.789,71$ & $94.789,71$ & $371,18 * *$ \\
C & 1 & $77.646,171$ & $77.646,171$ & $304,05 * *$ \\
D & 1 & $60.247,237$ & $60.247,237$ & $235,92 * *$ \\
E & 1 & $37.025,697$ & $37.025,697$ & $144,98 * *$ \\
F & 1 & $37.941,44$ & $37.941,44$ & $148,57 * *$ \\
AB & 1 & $8.788,7109$ & $8.788,7109$ & $34,41 * *$ \\
AC & 1 & $4.968,0471$ & $4.968,0471$ & $19,45 * *$ \\
AD & 1 & $3.768,3484$ & $3.768,3484$ & $14,76 * *$ \\
AE & 1 & $2.396,7756$ & $2.396,7756$ & $9,38 * *$ \\
AF & 1 & $2.139,0047$ & $2.139,0047$ & $8,38 * *$ \\
BC & 1 & $7.650,6729$ & $7.650,6729$ & $29,96 * *$ \\
BD & 1 & $1.833,6059$ & $1.833,6059$ & $7,18 *$ \\
BE & 1 & $1.637,2645$ & $1.637,2645$ & $6,41 *$ \\
BF & 1 & $4.200,0931$ & $4.200,0931$ & $16,45 * *$ \\
CD & 1 & $2.972,9075$ & $2.972,9075$ & $11,64 * *$ \\
CE & 1 & $3.692,0055$ & $3.692,0055$ & $14,46 * *$ \\
CF & 1 & $1.442,8127$ & $1.442,8127$ & $5,65 *$ \\
DE & 1 & $1.509,0797$ & $1.509,0797$ & $5,9 * *$ \\
DF & 1 & $1.682,179$ & $1.682,179$ & $6,59 *$ \\
EF & 1 & 678,70019 & 678,70019 & 2,66 \\
Residuo & 39 & $9.959,665$ & 255,37603 & - \\
\hline Tota1 & 63 & $504.118,68$ & & - \\
\hline
\end{tabular}


Os valores de $F$ são os mesmos do experimento 1.

Devemos notar que:

a. Em termos de significancia dos efeitos principais, e das interações nada se alterou.

b. 0 QM do residuo confundindo essas 2 intera . ções fica maior do que o $Q M$ do residuo sem confundimento, is to se deve a escolha das interações pois as suas SQ's são pequenas. Se fossem escolhidas as interações $A B E F$ e $A B C$ para serem confundidas, $O Q M$ do residuo diminuiria.

$$
\begin{aligned}
& \text { c. A eficiência será: } \\
& \hat{E}=\frac{n_{b} Q M_{B 1} \text { ocos }{ }^{+} n_{e} e_{E \text { Ero }}}{\left(n_{b}+n_{e}\right) Q M_{\text {Erro }}} \text {, onde } n_{b}=3 \text { e } n_{e}=63-3=60 \\
& \hat{\mathrm{E}}=\frac{3 \times 22,569678+60 \times 255,37603}{63 \times 255,37603}=\frac{15.390,271}{16.088,69} \rightarrow \hat{\mathrm{E}}=0,957
\end{aligned}
$$

Caso fossem escolhidas as interações $A B E F$ e $A B C$ para serem confundidas com efeito de blocos, teriamos:

$$
\begin{aligned}
& \mathrm{SQ}_{\mathrm{B} 1 \text { ocos }}=\mathrm{SQ}_{\mathrm{ABEF}}+\mathrm{SQ}_{\mathrm{ABC}}+\mathrm{SQ}_{\mathrm{CEF}} \rightarrow \mathrm{SQ}_{\mathrm{B} 1 \text { ocos }}=3.212,8368 \\
& \mathrm{SQ}_{\text {Res. }}=6.814,5372 \\
& \text { ou } \\
& \mathrm{QM}_{\mathrm{B} 1 \text { ocos }}=1.070,9456 \\
& \mathrm{QM}_{\text {Res. }}=174,73172
\end{aligned}
$$




$$
\begin{aligned}
& \text { A eficiência seria: } \\
& \widehat{\mathrm{E}}=\frac{3 \times 1.070,9456+60 \times 174,73172}{63 \times 174,73172}=\frac{13.696,74}{11.008,099} \rightarrow \widehat{\mathrm{E}}=1,244
\end{aligned}
$$

Comparando estas eficiēncias, vemos que seria melhor escolhermos as interações $A B E F$ e $A B C$ para serem confundidas, pois as mesmas dão uma eficiência maior do que 1. .

Agora vamos trabalhar com $1 / 2$ da repetição do experimento 3 .

Para isto vamos tomar a relação de identidade $I=+A B C D E F$, e assim temos as seguintes combinações de trata mento, ver exemplo 1 , do item 3.1.5.1.1.

$$
\begin{aligned}
& \text { (1) }=224,58 \quad \text { ae }=247,885 \\
& \text { af }=253,58 \quad \text { ef }=239,83 \\
& a b=291,01 \quad \text { be }=224,03 \quad \text { bf }=226,005 \text { abef }=375,25 \\
& \text { ac }=270,13 \quad \text { ce }=248,08 \quad \text { cf }=227,75 \quad \text { acef }=396,88 \\
& b c=262,805 \quad \text { abce }=437,495 \quad \text { abcf }=434,64 \quad \text { bcef }=371,18 \\
& \operatorname{ad}=281,4 \quad \mathrm{de}=244,065 \quad \mathrm{df}=271,975 \quad \text { adef }=383,22 \\
& \text { bd }=245,635 \text { abde }:=410,545 \quad \text { abdf }=428,75 \quad \text { bdef }=354,73 \\
& \text { cd }=248,565 \quad \text { acde }=401,905 \quad \text { acdf }=394,76 \quad \text { cdef }=326,355 \\
& \text { abcd }=435,71^{\circ} \text { bcde }=382,76 \quad \text { bcdf }=395,37 \text { abcdef }=636,115
\end{aligned}
$$

Aqui vamos utilizar o mesmo procedimento empregado para o experimento 1 , assim todas as observações dada anteriormente são vảlidas . 
No Quadro 6.(anexo 1), temos o quadro com a adaptação do procedimento de Yates. Assim o quado da anälise de variância é:
C. Variação
G.L.
S.Q.
$Q \cdot M$.
F

\begin{tabular}{crccc}
\hline Tratamentos & 21 & $277.423,34$ & - & - \\
A & 1 & $78.562,516$ & $78.562,516$ & $433,87 * *$ \\
B & 1 & $48.911,754$ & $48.911,754$ & $270,12 * *$ \\
C & 1 & $42.632,73$ & $42.632,73$ & $235,44 * *$ \\
D & 1 & $38.553,785$ & $38.553,785$ & $212,92 * *$ \\
E & 1 & $19.387,759$ & $19.387,759$ & $107,07 * *$ \\
F & 1 & $23.101,214$ & $23.101,214$ & $127,58 * *$ \\
AB & 1 & $4.715,176$ & $4.715,176$ & $26,04 * *$ \\
AC & 1 & $2.887,62$ & $2.887,62$ & $15,95 * *$ \\
AD & 1 & $1.517,1786$ & $1.517,1786$ & $8,38 *$ \\
AE & 1 & $1.390,8857$ & $1.390,8857$ & $7,68 *$ \\
AF & 1 & $1.181,4661$ & $1.181,4661$ & $6,52 *$ \\
BC & 1 & $5.838,2116$ & $5.838,2116$ & $32,24 * *$ \\
BD & 1 & $1.563,3834$ & $1.563,3834$ & $8,63 *$ \\
BE & 1 & 767,34031 & 767,34031 & 4,24 \\
BF & 1 & $1.304,4555$ & $1.304,4555$ & $7,20 *$ \\
CD & 1 & 37,044528 & 37,044528 & 0,20 \\
CE & 1 & $2.353,323$ & $2.353,323$ & $13,00 * *$ \\
CF & 1 & 539,64338 & 539,64338 & 2,98 \\
DE & 1 & 238,71125 & 238,71125 & 1,32 \\
DF & 1 & $1.534,4415$ & $1.534,4415$ & $8,47 *$ \\
EF & 1 & 404,70125 & 404,70125 & 2,23 \\
Residuo & 10 & $1.810,7525$ & 181,07525 & - \\
\hline Total & 31 & $279.234,09$ & & - \\
\hline
\end{tabular}




$$
\begin{aligned}
& F_{5 \%}(1,10)=4,96 \\
& F_{1 \%}(1,10)=10,04
\end{aligned}
$$

Com $1 / 2$ da repetição, notamos que:

a. Os efeitos principais e as interações $A B$, $A C, B C$ e $C E$ continuam sendo significativas aos niveis de $5 \%$ e $1 \%$, as interações $A D, A E, A F, B D, B F$ e $D F$ são significati- ' vas somente ao nivel de $5 \%$, enquanto as interações $B E, C D$, $C F, D E$ e EF são não significativas .

b. 0 seu QM do residuo diminuiu em relação ao QM do residuo do fatorial completo com e sem confundimento.

$$
\text { c. A eficiência será: }
$$

$$
\mathrm{E}=\frac{\mathrm{QM}_{\text {Res. comp } 1 .}}{\mathrm{QM}_{\text {Res.frac. }} \text { fract }}=\frac{238,747}{181,07525} \rightarrow \mathrm{E}=1,318
$$

Vemos que a eficiência do fatorial fracionado em relação ao fatorial completo é maior do que 1 .

$$
\text { Vamos agora colocar } 1 / 2 \text { do fatorial } 2^{6} \text { em. } 2 \text { blo }
$$
cos; para isto precisamos confundir uma interação de 3 fatores, e assim perdemos 1 g.1. do residuo.

A interação escolhida com sua respectiva alias 
serä $B C D=A E F$.

Para formar os 2 blocos procedemos da mesma maneira como no experimento 1.

$$
\text { Sabemos que a } \mathrm{SQ}_{\mathrm{B} 1 \text { ocos }}=\mathrm{SQ}_{\mathrm{BCD}}=1,3243781 \text {, e }
$$
a $\mathrm{SQ}_{\text {Rés. c/frac. e conf. }}=\mathrm{SQ}_{\mathrm{Res} \text {. c/frac. s/conf. }} \mathrm{SQ}_{\mathrm{BCD}}$, como também perdemos $1 \mathrm{~g} .1$. do residuo. o quadro da anälise. serā:

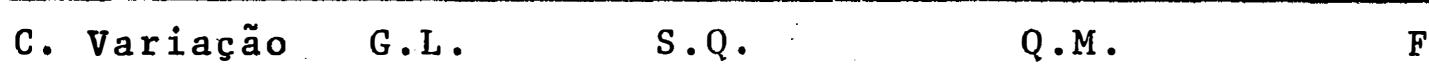

\begin{tabular}{|c|c|c|c|c|}
\hline B1ocos & 1 & 1,3243781 & - & - \\
\hline Tratamentos & 21 & $277.423,34$ & - & - \\
\hline A & 1 & $78.562,516$ & $78.562,516$ & $390,76 * *$ \\
\hline B & 1 & $48.911,754$ & $48.911,754$ & $243,28 * *$ \\
\hline $\mathrm{C}$ & 1 & $42.632,73$ & $42.632,73$ & $212,05 * *$ \\
\hline D & 1 & $38.553,785$ & $38.553,785$ & $191,76 * *$ \\
\hline $\mathrm{E}$ & 1 & $19.387,759$ & $19.387,759$ & $96,45 * *$ \\
\hline F & 1 & $23.101,214$ & $23.101,214$ & $114,90 * *$ \\
\hline$A B$ & 1 & $4.715,176$ & $4.715,176$ & $23,45 * *$ \\
\hline $\mathrm{AC}$ & 1 & $2.887,62$ & $2.887,62$ & $14,36 * *$ \\
\hline $\mathrm{AD}$ & 1 & $1.517,1786$ & $1.517,1786$ & $7,55 *$ \\
\hline $\mathrm{AE}$ & 1 & $1.390,8857$ & $1.390,8857$ & $6,92 *$ \\
\hline $\mathrm{AF}$ & 1 & $1.181,4661$ & $1.181,4661$ & $5,88 *$ \\
\hline $\mathrm{BC}$ & 1 & $5.838,2116$ & $5.838,2116$ & $29.04 * *$ \\
\hline BD & 1 & $1.563,3834$ & $1.563,3834$ & $7,78 *$ \\
\hline $\mathrm{BE}$ & 1 & 767,34031 & 767,34031 & 3,82 \\
\hline $\mathrm{BF}$ & 1 & $1.304,4555$ & $1.304,4555$ & $6,49 *$ \\
\hline CD & 1 & 37,044528 & 37,044528 & 0,18 \\
\hline $\mathrm{CE}$ & 1 & $2.353,323$ & $2.353,323$ & $11,71 * *$ \\
\hline CF & 1 & 539,64338 & 539,64338 & 2,68 \\
\hline $\mathrm{DE}$ & 1 & 238,71125 & 238,71125 & 1,19 \\
\hline $\mathrm{DF}$ & 1 & $1.534,4415$ & $1.534,4415$ & $7,63 *$ \\
\hline EF & 1 & 404,70125 & 404,70125 & 2,01 \\
\hline Residuo & 9 & $1.809,4281$ & 201,04757 & \\
\hline Tota 1 & 31 & $279.234,09$ & - & - \\
\hline
\end{tabular}




$$
\begin{aligned}
& F_{5 \%}(1,9)=5,12 \\
& F_{1 \%}(1,9)=10,56
\end{aligned}
$$

Notamos que:

a. Os efeitos principais continuam sendo alta mente significativos, e as interações $A D, A E, A F, B D$, ' $B F$ e $D F$ são significativas somente a nível de $5 \%$, enquanto as intera . ções $B E, C D, C F, D E$ e EF são não significativas.

b. 0 QM do residuo do fatorial fracionado com confundimento aumentou em relação ao mesmo sem confundimento, isto se deve a interação escolhida.

$$
\hat{\mathrm{E}}=\frac{\mathrm{QM} \text { Res. A eficiēncia serā: }}{\mathrm{QM}_{\text {Res. Frac. } / \text { conf } / \text { conf }}}=\frac{181,07525}{201,04757} \rightarrow \widehat{E}=0,901
$$

d. Caso fosse escolhida a interação $A B C$ (ver anexo 1 - Quadro 4), para ser confundida, O QM do residuo di minuiria, ou seja.

$$
\begin{aligned}
& \mathrm{SQ}_{\mathrm{B} 1 \text { ocos }}=\mathrm{SQ}_{\mathrm{ABC}}=708,76125, \mathrm{e} \\
& \mathrm{SQ}_{\text {Res.frac. c/conf. }}=1.101,9913 \text {, então: } \\
& \mathrm{QM}_{\text {Res.frac. c/conf. }}=\frac{1.101,9913}{9}=122,44348
\end{aligned}
$$


Assim, a eficiência neste caso será:

$$
\hat{\mathrm{E}}=\frac{\mathrm{QM}_{\text {Res.frac. } / \text { fonf. }}}{\mathrm{QM}_{\text {Res.frac. } \mathrm{c} / \mathrm{conf} .}}=\frac{181,07525}{122,44348} \rightarrow \mathrm{E}=1,479
$$

Comparando estas eficiências, vemos que seria melhor escolher a interação $A B C$ para ser confundida, pois ela nos dả uma eficiência maior do que 1 .

Agora vamos resumir o experimento 3 , montando - quadro abaixo, onde aparece os QM's residuais e as eficiên cias de todos casos vistos:

Experimento 3

Q.M. E

1. Fatorial completo s/conf.

2. Fatorial completo conf. a int. ABCDEF

3. Fatorial completo conf. a int. ABEF

4. Fatorial completo conf. as int's. ABDE e ACDF

5. Fatorial completo conf. as int's. ABEF e $A B C$

6. Fatorial fracionado: $1 / 2$ da rep. s/conf.

7. Fatorial fracionado: $1 / 2$ da rep. conf. a int. BCD 201,04757 0,901(*)

8. Fatorial fracionada $1 / 2$ da rep. conf. a int. ABC $122,443481,479(*)$

238,747

$231,92234 \quad 1,019$

$193,1467 \quad 1,157$

$255,37603 \quad 0,957$

$174,73172 \quad 1,244$

$181,07525 \quad 1,318$

(*) Estas eficiências foram calculadas sobre $1 / 2$ da repetição. 


\subsection{EXPER IMENTO 4}

A partir do Quadro 7 (ver anexo 1), obteve-se - seguinte quadro da anảise de variância para o fatorial completo:

C. Variação G.L. S.Q. Q.M.

\begin{tabular}{crccc}
\hline Tratamentos & 21 & $499.528,94$ & - & - \\
A & 1 & $142.798,96$ & $142.798,96$ & $333,78 * *$ \\
B & 1 & $96.362,128$ & $96.362,128$ & $225,24 * *$ \\
C & 1 & $78.185,946$ & $78.185,946$ & $182,75 * *$ \\
D & 1 & $59.871,973$ & $59.871,973$ & $139,94 * *$ \\
E & 1 & $33.115,811$ & $33.115,811$ & $77,40 * *$ \\
F & 1 & $37.751,518$ & $37.751,518$ & $88,24 * *$ \\
AB & 1 & $8.882,5913$ & $8.882,5913$ & $20,76 * *$ \\
AC & 1 & $4.742,0439$ & $4.742,0439$ & $11,08 * *$ \\
AD & 1 & $3.835,6346$ & $3.835,6346$ & $8,96 * *$ \\
AE & 1 & $2.370,9596$ & $2.370,9596$ & $5,54 *$ \\
AF & 1 & $2.458,4243$ & $2.458,4243$ & $5,73 *$ \\
BC & 1 & $8.837,4101$ & $8.837,4101$ & $20,66 * *$ \\
BD & 1 & $1.574,304$ & $1.574,304$ & 3,68 \\
BE & 1 & $1.589,4176$ & $1.589,4176$ & 3,72 \\
BF & 1 & $5.159,1898$ & $5.159,1898$ & $12,06 * *$ \\
CD & 1 & $2.785,9923$ & $2.785,9923$ & $6,51 *$ \\
CE & 1 & $4.151,5471$ & $4.151,5471$ & $9,70 * *$ \\
CF & 1 & $1.487,0664$ & $1.487,0664$ & 3,48 \\
DE & 1 & $1.239,9202$ & $1.239,9202$ & 2,90 \\
DF & 1 & $1.814,973$ & $1.814,973$ & $4,24 *$ \\
EF & 1 & 513,13576 & 513,13576 & 1,20 \\
Residuo & 42 & $17.968,605$ & 427,82393 & - \\
\hline Tota1 & 63 & $517.497,54$ & & - \\
\hline
\end{tabular}


Os valores de F são os mesmos do experimento 1 .

Vemos que os efeitos principais e as interações $A B, A C, A D, B C, B F$ e $C E$ são significativas aos niveis de $5 \%$ e $1 \%$, as interações $A E, A F, C D$ e $D F$ são significativas somente ao nive1 de $5 \%$, enquanto que as interações $B D, B E$, CF, DE e EF são não significativas.

Agora vamos confundir a interação de ordem mais elevada ou seja, ABCDEF. Para formar os 2 blocos proce demos da mesma maneira como no experimento 1.

Como a interação ABCDEF estā confundida, então a $\mathrm{SQ}_{\mathrm{B} 1 \text { ocos }}=\mathrm{SQ}_{\mathrm{ABCDEF}}=999,35016$, e a $S Q_{\text {Res. }} \mathrm{c/conf} .=$ $=\mathrm{SQ}_{\text {Res. }}$ s/conf. $-\mathrm{SQ}_{\mathrm{ABCDEF}}$, como também se perde $1 \mathrm{~g} \cdot 1$. do residuo. 0 quadro da anảlise de variância será: 


\begin{tabular}{|c|c|c|c|c|}
\hline C. Variação & $G \cdot L$ & $S \cdot Q \cdot$ & $Q \cdot M$ & $\mathrm{~F}$ \\
\hline Blocos & 1 & $999 ; 35016$ & - & - \\
\hline Tratamentos & 21 & $499.528,94$ & - & - \\
\hline A & 1 & $142.798,96$ & $142.798,96$ & $345,02 * *$ \\
\hline B & 1 & $96.362,128$ & $96.362,128$ & $232,82 * *$ \\
\hline $\mathrm{C}$ & 1 & $78.185,946$ & $78.185,946$ & $188,91 * *$ \\
\hline $\mathrm{D}$ & 1 & $59.871,973$ & $59.871,973$ & $144,66 * *$ \\
\hline $\mathrm{E}$ & 1 & $33.115,811$ & $33.115,811$ & $80,01 * *$ \\
\hline $\mathbf{F}$ & 1 & $37.751,518$ & $37.751,518$ & $91,21 * *$ \\
\hline$A B$ & 1 & $8.882,5913$ & $8.882,5913$ & $21,46 * *$ \\
\hline $\mathrm{AC}$ & 1 & $4.742,0439$ & $4.742,0439$ & $11,46 * *$ \\
\hline$A D$ & 1 & $3.835,6346$ & $3.835,6346$ & $9,27 * *$ \\
\hline $\mathrm{AE}$ & 1 & $2.370,9596$ & $2.370,9596$ & $5,73 *$ \\
\hline $\mathrm{AF}$ & 1 & $2.458,4243$ & $2.458,4243$ & $5,94 *$ \\
\hline $\mathrm{BC}$ & 1 & $8.837,4104$ & $8.837,4104$ & $21,35 * *$ \\
\hline $\mathrm{BD}$ & 1 & $1.574,304$ & $1.574,304$ & 3,80 \\
\hline $\mathrm{BE}$ & 1 & $1.589,4176$ & $1.589,4176$ & 3,84 \\
\hline B F & 1 & $5.159,1898$ & $5.159,1898$ & $12,46 * *$ \\
\hline$C D$ & 1 & $2.785,9923$ & $2.785,9923$ & $6,73 *$ \\
\hline $\mathrm{CE}$ & 1 & $4.151,5471$ & $4.151,5471$ & $10,03 * *$ \\
\hline $\mathrm{CF}$ & 1 & $1.487,0664$ & $1.487,0664$ & 3,59 \\
\hline $\mathrm{DE}$ & 1 & $1.239,9202$ & $1.239,9202$ & 3,00 \\
\hline $\mathrm{DF}$ & 1 & $1.814,973$ & $1.814,973$ & $4,38 *$ \\
\hline $\mathrm{EF}$ & 1 & 513,13576 & 513,13576 & 1,24 \\
\hline Residuo & 41 & $16.969,255$ & 413,88427 & - \\
\hline Total & 63 & $517.497,54$ & - & - \\
\hline
\end{tabular}

Os valores de $F$ são os mesmos do experimento 1 . 
Podemos observar que:

a. Com o confundimento da interação ABCDEF, em termos de significância não mudou nada.

b. 0 QM do residuo com confundimento fica menor do que o QM do residuo sem confundimento, e estadiferen ça è de 13,93966.

c. Esta diferença entre os QM's. serä maior ou menor pela escolha da interação a ser confundida, : por exemplo, se escolhessemos a interação $A B E F, O \cdot Q M$ do resíduo seria 343,06809. Mas, se fosse escolhida a interação ACDF, - QM do residuo seria 438,25333, como vemos o mesmo aumentou em relação ao QM do residuo do fatorial completo.

$$
\begin{aligned}
& \text { d. A eficiēncia será: } \\
& \overline{\mathrm{E}}=\frac{\mathrm{n}_{b} \mathrm{M}_{B 1 \text { ocos }}+\mathrm{n}_{e} \mathrm{MM}_{\text {Erro }}}{\left(\mathrm{n}_{b}+\mathrm{n}_{e}\right){ }_{\text {Erro }}} \text {, onde } \\
& \mathrm{n}_{\mathrm{b}}=1 \\
& \mathrm{n}_{\mathrm{e}}=63-1=62 \text {, então } \\
& \widehat{\mathrm{E}}=\frac{1 \times 999,35016+62 \times 413,88427}{63 \times 413,88427}=\frac{26.660,175}{26.074,709} \rightarrow \widehat{E}=1,022
\end{aligned}
$$


e. Se a interação escolhida fosse ABEF, a ef $\underline{i}$ ciência seria:

$$
\begin{gathered}
\mathrm{QM}_{\text {B1ocos }}=3.902,8133 \\
\mathrm{QM}_{\text {Erro }}=\frac{14.065,792}{41}=343,06809 \\
\widehat{\mathrm{E}}=\frac{1 \times 3.902,8133+62 \times 343,06809}{63 \times 343,06809}=\frac{25.173,035}{21.613,29} \rightarrow \widehat{E}=1,165 .
\end{gathered}
$$

Como foi visto para os experimentos 1,2 e 3, a eficiência da interação $A B E F$ quando confundida, continua sendo maior do que a interaça o $A B C D E F$.

Agora vamos confundir as interações $A B D E$ e ACDF, como sabemos a interação generalizada BCEF também estarä confundida.

Para formar os 4 blocos procedemos da mesma maneira como no experimento 1 .

Como as interações $\mathrm{ABDE}$, ACDF e BCEF estão con fundidas, então a $\mathrm{SQ}_{\mathrm{B} 1 \text { ocos }}=\mathrm{SQ}_{\mathrm{ABDE}}+\mathrm{SQ}_{\mathrm{ACDF}}+\mathrm{SQ}_{\mathrm{BCEF}}=31,276056$ $+0,2185562+93,170756 \rightarrow \mathrm{SQ}_{\mathrm{B} 1 \text { ocos }}=124,66537$, então:

$\mathrm{SQ}_{\text {Res. }} \mathrm{c} / \mathrm{conf} .=\mathrm{SQ}_{\operatorname{Res} . \mathrm{s} / \mathrm{conf} .}-\mathrm{SQ}_{\mathrm{B} 1 \text { ocos }}=17.968,605$ $-124,66537 \rightarrow$

$$
\mathrm{SQ}_{\text {Res. }} \mathrm{c} / \mathrm{conf} .=17.843,94
$$


Assim, temos o quadro da anälise de variância.

\begin{tabular}{|c|c|c|c|c|}
\hline C. Variação & G.L. & $S \cdot Q$. & $\mathrm{Q} \cdot \mathrm{M}$. & $F$ \\
\hline B1ocos & 3 & 124,66537 & 41,555123 & - \\
\hline Tratamentos & 21 & $499.528,94$ & - & - \\
\hline A & 1 & $142.798,96$ & $142.798,96$ & $312,10 * *$ \\
\hline B & 1 & $96.362,128$ & $96.362,128$ & $210,61 * *$ \\
\hline $\mathrm{C}$ & 1 & $78.185,946$ & $78.185,946$ & $170,88 * *$ \\
\hline D & 1 & $59.871,973$ & $59.871,973$ & $130,86 * *$ \\
\hline E & 1 & $33.115,811$ & $33.115,811$ & $72,38 * *$ \\
\hline F & 1 & $37.751,518$ & $37.751,518$ & $82,51 * *$ \\
\hline$A B$ & 1 & $8.882,5913$ & $8.882,5913$ & $19,41 * *$ \\
\hline $\mathrm{AC}$ & 1 & $4.742,0439$ & $4.742,0439$ & $10,36 * *$ \\
\hline $\mathrm{AD}$ & 1 & $3.835,6346$ & $3.835,6346$ & $8,38 * *$ \\
\hline $\mathrm{AE}$ & 1 & $2.370,9596$ & $2.370,9596$ & $5,18 *$ \\
\hline $\mathrm{AF}$ & 1 & $2.458,4243$ & $2.458,4243$ & $5,37 *$ \\
\hline $\mathrm{BC}$ & 1 & $8.837,4101$ & $8.837,4101$ & $19,32 * *$ \\
\hline B D & 1 & $1.574,304$ & $1.574,304$ & 3,44 \\
\hline $\mathrm{BE}$ & 1 & $1.589,4176$ & $1.589,4176$ & 3,47 \\
\hline B F & 1 & $5.159,1898$ & $5.159,1898$ & $11,28 * *$ \\
\hline CD & 1 & $2.785,9923$ & $2.785,9923$ & $6,09 *$ \\
\hline $\mathrm{CE}$ & 1 & $4.151,5471$ & $4.151,5471$ & $9,07 * *$ \\
\hline CF & 1 & $1.487,0664$ & $1.487,0664$ & 3,25 \\
\hline $\mathrm{DE}$ & 1 & $1.239,9202$ & $1.239,9202$ & 2,71 \\
\hline $\mathrm{DF}$ & 1 & $1.814,973$ & $1.814,973$ & 3,97 \\
\hline EF & 1 & $\quad 513,13576$ & 513,13576 & 1,12 \\
\hline Residuo & 39 & $17.843,94$ & 457,53692 & - \\
\hline Tota 1 & 63 & $517.497,54$ & - & - \\
\hline
\end{tabular}

Os valores de $F$ são os mesmos do experimento 1. 
Devemos notar que:

a. Os efeitos principais e as interações AB, $A C, A D, B C, B F$ e $C E$ continuam sendo significativas aos niveis de $5 \%$ e $1 \%$

b. $0 Q M$ do residuo confundindo essas 2 intera ções fica maior do que o $Q M$ do residuo sem confundimento, is to se deve a escolha das interações, pois as suas SQ's são pequenas. Se fossem escolhidas as interações $A B E F$ e $A B C$ para serem confundidas, o $Q M$ do erro diminuiria.

$$
\begin{aligned}
& \text { c. A eficiēncia serä: }
\end{aligned}
$$

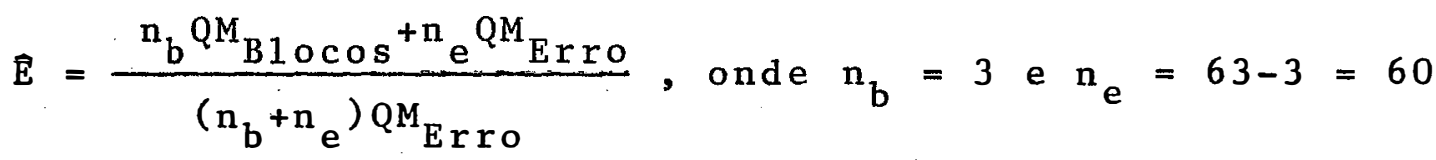

$$
\begin{aligned}
& \hat{E}=\frac{3 \times 41,555123+60 \times 457,53692}{63 \times 457,53692}=\frac{27.576,881}{28.824,826} \rightarrow \hat{E}=0,957
\end{aligned}
$$

Caso fosse escolhidas as interações $A B E F$ e ABC para serem confundidas com efeitos de blocos teriamos:

$$
\begin{aligned}
& \mathrm{SQ}_{\mathrm{B} 1 \text { ocos }}=\mathrm{SQ}_{\mathrm{ABEF}}+\mathrm{SQ}_{\mathrm{ABC}}+\mathrm{SQ}_{\mathrm{CEF}} \rightarrow \mathrm{SQ}_{\mathrm{B} 1 \text { ocos }}=5.785,3287 \\
& \mathrm{SQ}_{\text {Res. }}=12.183,276
\end{aligned}
$$

ou 


$$
\begin{aligned}
& \mathrm{QM}_{\mathrm{B} 1 \text { ocos }}=1.928,4429 \\
& \mathrm{QM}_{\text {Res. }}=312,3917
\end{aligned}
$$

A eficiēncia seria:

$$
E=\frac{3 \times 1.928,4429+60 \times 312,3917}{63 \times 312,3917}=\frac{24.528,831}{19.680,677} \rightarrow \quad \hat{E}:=1,246
$$

Comparando estas eficièncias, vemos que seria melhor escolhermos as interações $A B E F$ e $A B C$ para serem confundidas, pois as mesmas dão uma eficiência maior do que 1 .

Agora vamos trabalhar com $1 / 2$ da repetição do experimento 4 .

Para isto vamos tomar a relação de identidade $I=+A B C D E F$, e assim temos as seguintes combinações de tratamentos, ver exemplo 1, do item 3.1.5.1.1.
(1) $=223,14$
$\mathrm{ae}=242,68$
af $=250,94$
ef $=237,14$.
$\mathrm{ab}=291,98$
be $=215,54$
$\mathrm{bf}=218,84$
abef $=369,30$
$\mathrm{ac}=268,84$
ce $=248,24$
cf $=221,80$
acef $=402,84$
$\mathrm{bc}=263,74$
abce $=441,86$
$a b c f=440,72$
bcef $=373,24$
$\mathrm{ad}=286,20$
$\mathrm{de}=242,62$
$\mathrm{df}=280,50$
adef $=386,96$
bd $=243,18$
abde $=410,86$
$\operatorname{abdf}=437,80$
bdef $=353,64$
cd $=247,72$
acde $=394,04$
$\operatorname{acdf}=397,18$
cdef $=316,44$
$\mathrm{abcd}=434,98$
bcde $=383,18$
bcdf $=402,66$
abcdef : $=638,52$

Aquí vamos utilizar o mesmo procedimento em- 
pregado para o experimento 1 , assim todas as observaços dada anteriormente são välidas.

No Quadro 8 (anexo 1) temos o quadro com

$\mathbf{a}$ adaptação do procedimento de Yates. Assim o quadro da anäli se de variância è :
C. Variação
G . L .
S . Q •
Q.M.
F

\begin{tabular}{|c|c|c|c|c|}
\hline Tratamentos & 21 & $289.195,73$ & & \\
\hline $\mathrm{A}$ & 1 & $82.426,12$ & $82.426,12$ & $272,70 * *$ \\
\hline B & 1 & $50.622,438$ & $50.622,438$ & $167,48 * *$ \\
\hline C & 1 & $43.858,334$ & $43.858,334$ & $145,10 * *$ \\
\hline D & 1 & $41.015,344$ & $41.015,344$ & $135,69 * *$ \\
\hline $\mathrm{E}$ & 1 & $17.432,179$ & $17.432,179$ & $57,67 * *$ \\
\hline F & 1 & $24.737,552$ & $24.737,552$ & $81,84 * *$ \\
\hline $\mathrm{AB}$ & 1 & $4.998,0002$ & $4.998,0002$ & $16,54 * *$ \\
\hline $\mathrm{AC}$ & 1 & $2.809,5008$ & $2.809,5008$ & $9,29 *$ \\
\hline $\mathrm{AD}$ & 1 & $1.366,5992$ & $1.366,5992$ & 4,52 \\
\hline $\mathrm{AE}$ & 1 & $1.377,6001$ & $1.377,6001$ & 4,56 \\
\hline $\mathrm{AF}$ & 1 & $1.456,9202$ & $1.456,9202$ & 4,82 \\
\hline BC & 1 & $7.528,872$ & $7.528,872$ & $24,91 * *$ \\
\hline BD & 1 & $1.704,6961$ & $1.704,6961$ & $5,64 *$ \\
\hline BE & 1 & 776,18 & 776,18 & 2,57 \\
\hline $\mathrm{BF}$ & 1 & $1.366,0765$ & $1.366,0765$ & 4,52 \\
\hline$C D$. & 1 & 46,94805 & 46,94805 & 0,16 \\
\hline $\mathrm{CE}$ & 1 & $2.711,4248$ & $2.711,4248$ & $8,97 *$ \\
\hline $\mathrm{CF}$ & 1 & 543,51045 & 543,51045 & 1,80 \\
\hline $\mathrm{DE}$ & 1 & 63,845 & 63,845 & 0,21 \\
\hline $\mathrm{DF}$ & 1 & $1.986,3905$ & $1.986,3905$ & $6,57 *$ \\
\hline $\mathrm{EF}$ & 1 & 367,205 & 367,205 & 1,21 \\
\hline Residuo & 10 & $3.022,6156$ & 302,26156 & - \\
\hline Tota 1 & 31. & $292.218,35$ & - & \\
\hline
\end{tabular}

$$
\begin{aligned}
& F_{5 \%}(1,10)=4,96 \\
& F_{1} \%(1,10)=10,04
\end{aligned}
$$


Com $1 / 2$ da repetição, notamos que:

a. Os efeitos principais e as interações $A B$ e BC continuam sendo significativas aos niveis de $5 \%$ e $1 \%$, as interações AC, BD, CE e DF são significativas somente ao nive1 de 5\%, enquanto que as interações $A D, A E, A F, B E, \quad B F$, $C D, C F, D E$ e EF são não significativas .

b. o seu QM de resíduo diminuiu em relação ao QM de residuo do fatorial completo com e sem confundimento.

c. A eficiência serä:

$$
\widehat{\mathrm{E}}=\frac{\mathrm{QM}_{\text {Res.comp } 1 .}}{\mathrm{QM}_{\text {Res.frac. }}} \frac{427,82393}{302,26156} \rightarrow \widehat{\mathrm{E}}=1,415
$$

Vemos que a eficiēncia do fatorial fracionado em relação ao fatorial completo è maior do que 1 .

Vamos agora colocar $1 / 2$ do fatorial $2^{6}$ em 2 blocos, para isto precisamos confundir uma interaça de 3 fa tores, e assim perdemos $1 \mathrm{~g} .1$. do residuo.

A interação escolhida com sua respectiva alias serä $B C D=A E F$.

Para formar os dois blocos procedemos da mes- 
ma maneira como no experimento 1.

$$
\text { Sabemos que a } \mathrm{SQ}_{\mathrm{Blocos}}=\mathrm{SQ}_{\mathrm{BCD}}=10,90445 \text {, e }
$$

${ }^{S Q_{\text {Res. }}}$ c/frac. e conf. $=\mathrm{SQ}_{\mathrm{Res}}$. c/frac. s/conf. $-\mathrm{SQ}_{\mathrm{BCD}}$, como também perdemos 1 g. 1 . do residuo. o quadro da änälise de variāncia ser:

$\begin{array}{llll}\text { C. Variação G.L. S.Q. } & \text { Q.M. } & \text { F }\end{array}$

\begin{tabular}{|c|c|c|c|c|}
\hline B1ocos & 1 & 10,90445 & - & - \\
\hline Tratamentos & 21 & $289.195,73$ & - & - \\
\hline $\mathrm{A}$ & 1 & $82.426,12$ & $82.426,12$ & $246,32 * *$ \\
\hline B & 1 & $50.622,438$ & $50.622,438$ & $151,28 * *$ \\
\hline C & 1 & $43.858,334$ & $43.858,334$ & $131,06 * *$ \\
\hline D & 1 & $41.015,344$ & $41.015,344$ & $122,57 * *$ \\
\hline $\mathrm{E}$ & 1 & $17.432,179$ & $17.432,179$ & $52,09 * *$ \\
\hline F & 1 & $24.737,552$ & $24.737,552$ & $73,92 * *$ \\
\hline$A B$ & 1 & $4.998,0002$ & $4.998 ; 0002$ & $14,94 * *$ \\
\hline $\mathrm{AC}$ & 1 & $2.809,5008$ & $2.809,5008$ & $8,40 *$ \\
\hline $\mathrm{AD}$ & 1 & $1.366,5992$ & $1.366,5992$ & 4,08 \\
\hline $\mathrm{AE}$ & 1 & $1.377,6001$ & $1.377,6001$ & 4,12 \\
\hline $\mathrm{AF}$ & 1 & $1.456,9202$ & $1.456,9202$ & 4,35 \\
\hline $\mathrm{BC}$ & 1 & $7.528,872$ & $7.528,872$ & $22,50 * *$ \\
\hline BD & 1 & $1.704,6961$ & $1.704,6961$ & 5,09 \\
\hline $\mathrm{BE}$ & 1 & 776,18 & 776,18 & 2,32 \\
\hline $\mathrm{BF}$ & 1 & $1.366,0765$ & $1.366,0765$ & 4,08 \\
\hline CD̀ & 1 & 46,94805 & 46,94805 & 0,14 \\
\hline CE & 1 & $2.711,4248$ & $2.711,4248$ & $8,10 *$ \\
\hline $\mathrm{CF}$ & 1 & 543,51045 & 543,51045 & 1,62 \\
\hline $\mathrm{DE}$ & 1 & 63,845 & 63,845 & 0,19 \\
\hline $\mathrm{DF}$ & 1 & $1.986,3905$ & $1.986,3905$ & $5,94 *$ \\
\hline $\mathrm{EF}$ & 1 & 367,205 & 367,205 & 1,10 \\
\hline Residuo & 9 & $3.011,7112$ & 334,63458 & - \\
\hline
\end{tabular}

Tota 1

$31292.218,35$

$\mathrm{F}_{5 \%}(1,9)=5,12$

$F_{1 \%}(1,9)=10,56$ 
Notamos que:

a. Os efeitos principais e as interaçós $A B$ e BC continuam sendo significativas aos niveis de $5 \%$ e $1 \%$.

b. A interação BD que seu confundimento era significativa ao níve1 de $5 \%$, aqui ela não é mais, isto se deve ao aumento do QM do residuo.

c. Como jä sabemos pelo item b, o QM do residuo do fatorial fracionado com confundimento aumentou em relação ao mesmo sem confundimento, isto se deve a interaça escolhida.

d. A eficiência serà:

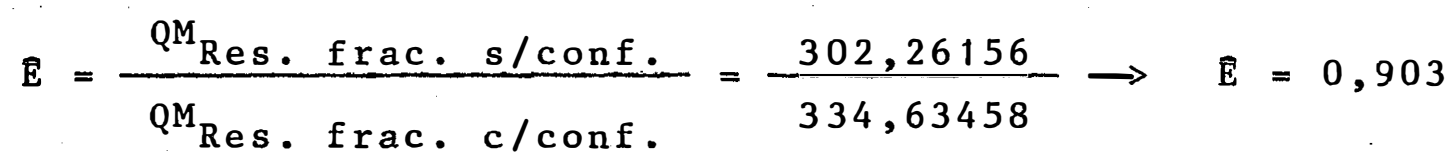

e. Caso fosse escolhida a interação $A B C$ (ver anexo 1.- Quadro 8), para ser confundida, o QM do residuo dí minuiria, ou seja:

$S Q_{B 1 \text { ocos }}=S_{A B C}=1.137,645, \mathrm{e}$

$\mathrm{SQ}_{\text {Res. frac. }}$ / $/$ conf. $=1.884,9706$, então:

$\mathrm{QM}_{\text {Res. frac. } \mathrm{c} / \mathrm{conf} .}=\frac{1.884,9706}{9}=209,44118$ 
Assim, a eficiência neste caso serä:

$$
\overline{\mathrm{E}}=\frac{\mathrm{QM}_{\text {Res.frac. s/conf. }}}{\mathrm{QM}_{\text {Res.frac. c/conf. }}}=\frac{302,26156}{209,44118} \rightarrow \overline{\mathrm{E}}=1,443
$$

Comparando estas eficiências, vemos que seria melhor escolher a interação ABC para ser confundida, pois ela nos dá uma eficiència maior do que 1 .

Agora vamos resumir o experimento 4, montando - quadro abaixo, onde aparece os QM's residuais e as eficiên cias de todos os casos vistos:

1. Fatorial completo s/conf.

2. Fatorial completo conf. a int. ABCDEF

3. Fatorial completo conf. a int. ABEF

4. Fatorial completo conf. as int's. ABDE e ACDF

5. Fatorial completo conf. as int's. ABEF e $A B C$

6. Fatorial fracionado: $1 / 2$ da rep. s/conf.

7. Fatorial fracionado: $1 / 2$ da rep. conf. a int. BCD

8. Fatorial fracionado: $1 / 2$ da rep. conf. a int. ABC 209,44118 1,443(*)
$427,82393=$

$413,88427 \cdot 1,022$

$343,068091,165$

$457,536920,957$

$312,3917 \quad 1,246$

$302,261561,415$

$334,634580,903(*)$

(*) Estas eficiências foram calculadas sobre $1 / 2$ da repetição. 
No quadro abaixo, temos os resultados dos 4 experimentos, dando para cada um os QM'S e as eficiència dos casos estudados.

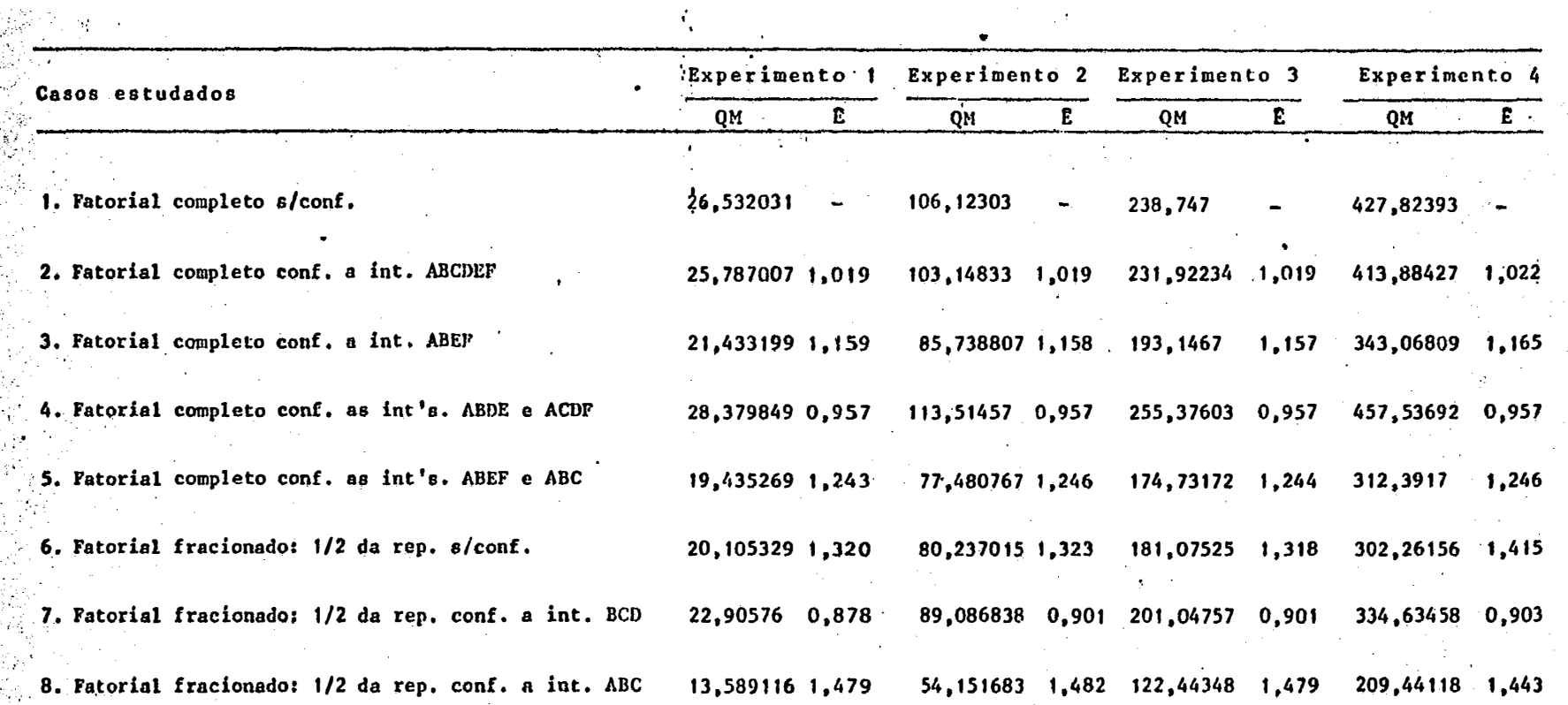


Tomando como base este quadro, podemos notar que aumentando os desvios padrões dos erros observamos que:

a. Os QM's aumentam ou diminuem na mesma proporção nos 8 casos estudados em cada experimento.

b. As eficiências não mudam de um experimento para outro.

c. 0 fatorial fracionado em cada experimento tem o seu $Q M$ do residuo menor do que o $Q M$ do residuo fatorial completo sem confundimento, e com isto a eficiēncia è maior do que 1 .

d. Dependendo da interação escolhida para ser confundida, o QM do residuo tanto para fatorial completo como para o fatorial fracionado pode aumentar ou diminuir.

e. 0 menor $Q M$ do residuo e consequentemente a maior eficiência do fatorial completo foi quando se confun diu as interações $A B E F$ e $A B C$, e para o fatorial fracionado foi quando a interação $A B C$ foi confundida, isto não muda para os 4 experimentos. 


\section{CONCLUSÕES}

Pelos resultados e comentärios obtidos anteriormente, podemos chegar a algumas conclusões importantes sobre os 4 experimentos discutidos.

5.1. Quando os desvios padrões dos erros aumentam, temos:

a. Para o fatorial completo com e sem confundimento, algumas interações de 2 fatores passam a ser significativas somente ao nível de $5 \%$, e outras passam a ser não significativas.

b. Para $1 / 2$ da repetição com e sem confundimento, somente no primeiro experimento é que todos os efeitos principais e interações de 2 fatores são significativos aos nîveis de $5 \%$ e $1 \%$. 
5.2. A eficiência do fatorial fracionado sem confundimento em relação ao fatorial completo sem confundimento é maior do que 1 , nos 4 experimentos estudados.

5.3. Diminuição do $Q M$ do resíduo tanto para o fatorial completo como para o fatorial fracionado depende da interação escolhida para ser confundida.

5.4. Para os fatoriais da série $3^{n}$, o procedi mento é o mesmo, ou seja, será uma extensão do que ocorre com os fatoriais da série $2^{n}$. 


\section{BIBLIOGRAFIA}

ADDELMAN, S., 1963. Tecniques for constructing fractional replicate plans. Journal American Statistical Association, 58: $45-71$.

BANERJEE, K.S., 1950. A note on fractional replication of factorial arrangements. Sankhyã: The Indian Journal of Statistics, 10: 87-94.

BANERJEE, K,S, e W.T, FEDERER, 1966. On estimation and constructio in fractional replication. Annals Mathematical Statistics, 37: 1033-1039.

BIAZI, E., 1985. Construção de frações de fatoriais de reso lução III. Piracicaba, ESALQ, 196 p. (Dissertação de Mes trado). 
BOSE, R.C. e K. KISHEN, 1940. On the Problem of confounding in the general symmetric factorial design. Sankhyã The Indian Journal of Statistics, $\underline{5}$ : 21-36.

BROWNLEE, K.A., B.K. KELLEY A P.K. LORRAINE, 1948. Fractional replication arrangements for factorial experiments with factors at two levels.. Biometrika, XXXV, III e IV: 268-276.

CHINLOY, T., R.F. INNES e D.J. FINNEY, 1952. An example of fractional replication in an experiment on sugar cane manuring. Journal of Agricultural Science, 43: 1-11.

COCHRAN, W.G. e G.M. COX, 1957. Experimental Designs. John Willey and Sons, 2a' Edição, Nova York. 611 p.

DAVIES, O.L. e W.A. HAY, 1950. The construction and uses of fractional factorial designs in industrial research. Biometrics, 6 : 233-249.

FINNEY, D.J., 1945. The fractional replication of factorial arrangements. Annals of eugenies, Lond., 12,4:291-301.

FINNEY, D.J., 1945. Recent developments in the design of field experiments. III. Fractional replication. Journal of Agricultural seience, 36,3: 184-191.

FINNEY, D.J., 1947. The construction of confounding arrangements. Empire Journal Experimental Agricultural, 15: 107-112. 
FISHER, R.A., 1942. The Theory of confounding in factorial experiments in relation to the theory of groups. Annal of Eugenies, Lond., 11: 341-353.

FISHER, R.A., 1945. A system of confounding for factors with more than two alternatives, giving completely orthogonal cubes and higher powers. Annals of Eugenics, Lond., 12: 283-290.

FISHER, R.A., 1951. The Design of Experiments. 01 iver and Boyd, London, 6. Edição. 244 p.

JOHN, P.W.M., 1962. Three quarter replicates of $2^{\text {n }}$ designs. Biometrics, 18: 172-184.

KEMPTHORNE, O., 1947. A note on differential responses in blocks. Journal of Agricultural Science, 37: 245-248.

KEMPTHORNE, 0., 1947. A simple approach to confounding and fractional replication in factorial experiments. Biometrika, XXXIV, III and IV: 255-272.

KEMPTHORNE, O.je R.G. TISCHER, 1953: An example of the use of fractional replication. Biometrics, 9: 295-303.

KEMPTHORNE, 0., 1975. The Design and Analysis of Experiments. 6. Edição. John Wiley, Nova York, $631 \mathrm{p}$. 
KISHEN, K., 1942. On expressing any single degree of freedon for treatment in $s^{m}$ factorial arrangement in terms of its sets for main effects and interactions. Sankhyã: The Indian Journal of Statistics, 6: 133-140.

KSHIRSAGAR, A.M., 1966. Balanced factorial designs. Journal of the Royal Statistical Society, 28B-3: 559-567.

KURKJIAN; B. e M. ZELEN, 1962. A calculus of factorial arrangements. Annalu Mathematical statistics, 33: 600-619.

KURKJIAN, B. e M. ZELEN, 1963. Applications of the calculus of factorial arrangements. I. Block and direct product designs. Biometrika, 50: 63-73.

LIMA, I.V., 1985.. Obtenção de frações de fatoriais de reso1 ução V. Piracicaba, ESALQ, 142 p. (Dissertação de Mestrado).

MULLER, E.R., 1966. Balanced confounding of factorial experiments. Biometrika, 53,3 e 4: 507-524.

NETER, J. e WASSERMAN, W., 1974. Applied Linear statistical Models. 1. Edição. Richard D. Irwin, Inc. 842 p. PLACKETT, R.L. e J.P. BURMAN, 1946. The design of optimum multifractional experiments. Biometrika, XXXIII, IV: $305-325$. 
RAGHAVARAD, D., 1971.' Constructions and Combinatorial Problems in Design of Experiments. John Wiley and Sons, Nova York, $386 \mathrm{p}$.

RAO, C.R., 1948. The Theory of fractional replication in factorial experiments. Sankhyã: The Indian Journal of Statistics, 10: 81-86.

YATES, F., 1937. The Design and analysis of factorial experiments. Technïcal Communication, n! 35. Imperial Burean of soil science.

ZONTA, E.P., 1980. Um. método de confundimento nos experimen tos fatoriais. Piracicaba, ESALQ, 176 p. (Dissertação de Mestrado). 
.183.

A N E X 01 


\section{$\mid \begin{aligned} & 0 \\ & 0 \\ & 0 \\ & 0 \\ & 0 \\ & 0 \\ & 0 \\ & 0 \\ & \vdots \\ & 0 \\ & 0 \\ & 0 \\ & 0 \\ & 0\end{aligned}$ \\ a \\ ฐ in}

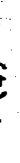

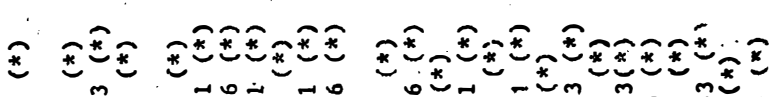

กิ กิํํำ o수ำ

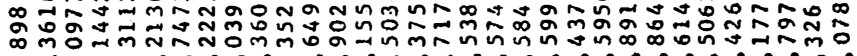

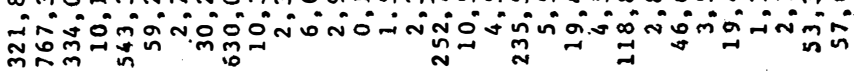

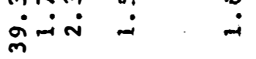

等

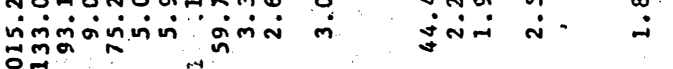

$\therefore$

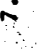

山ᄄ口

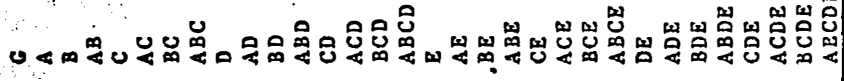

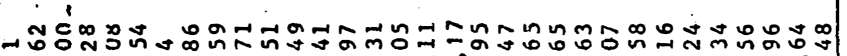
son inin

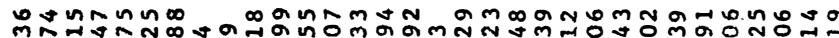

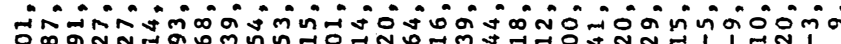
ณ

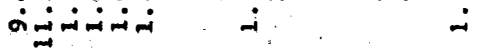

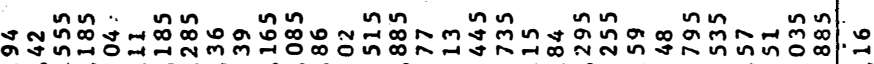
จ

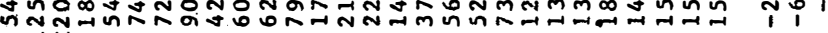
ininum.

ำกำกำ

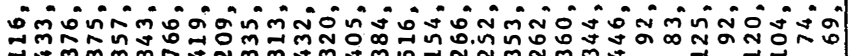
ivivivinim

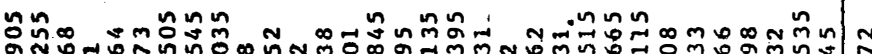

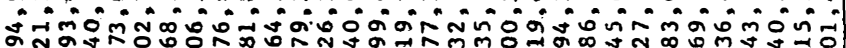

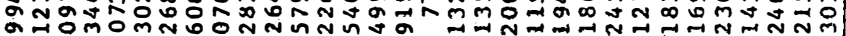
-i-i-i-i-i-i-i-i

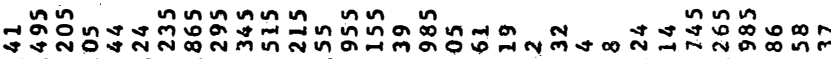

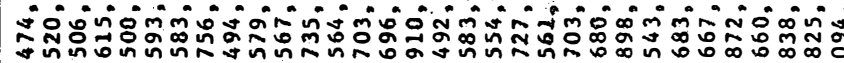

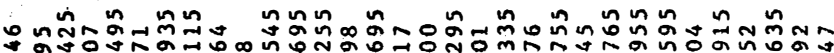

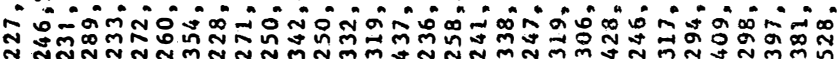

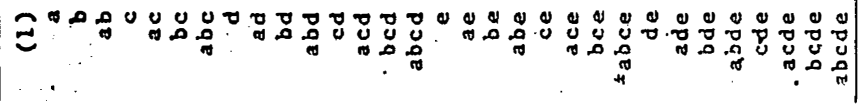

ก⿻上丨丶 วฆ์

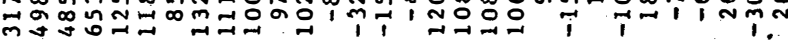

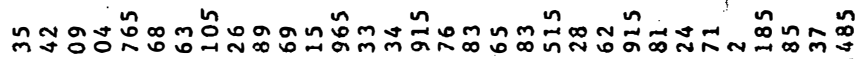

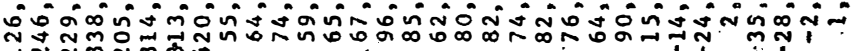

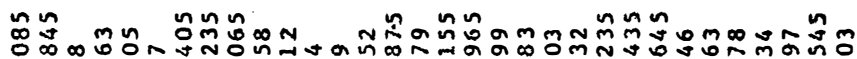

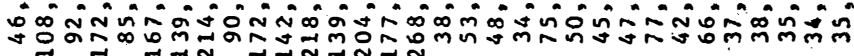

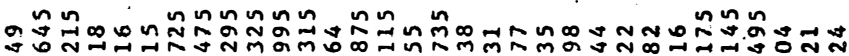

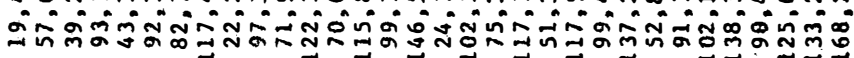

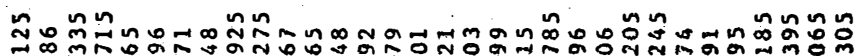

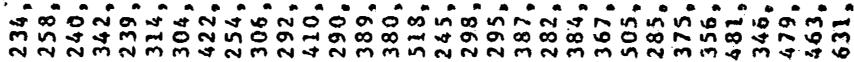

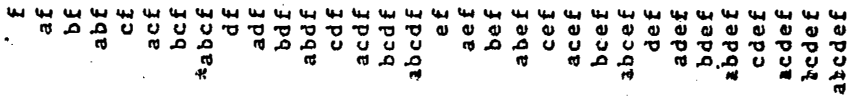


.185 .

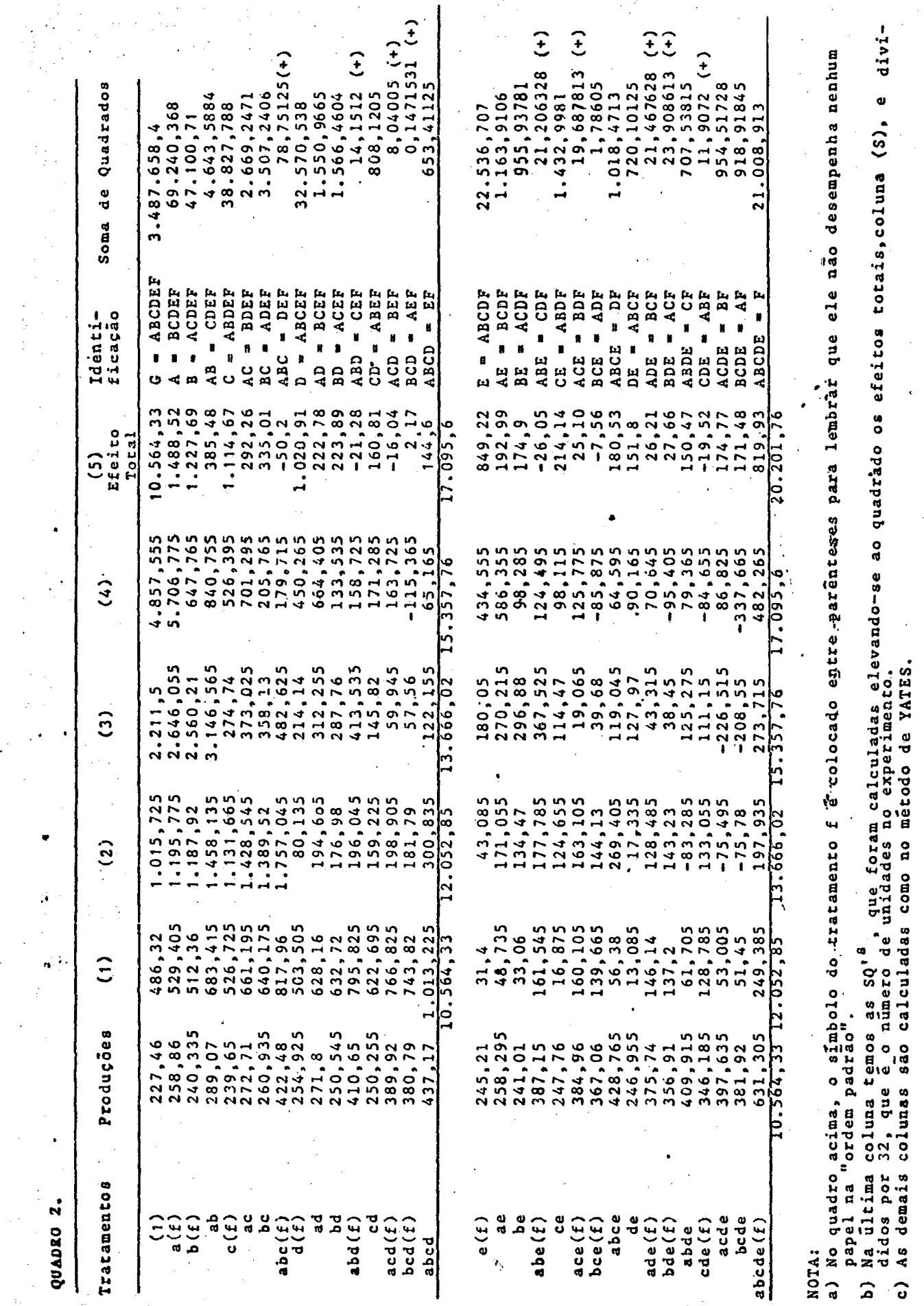




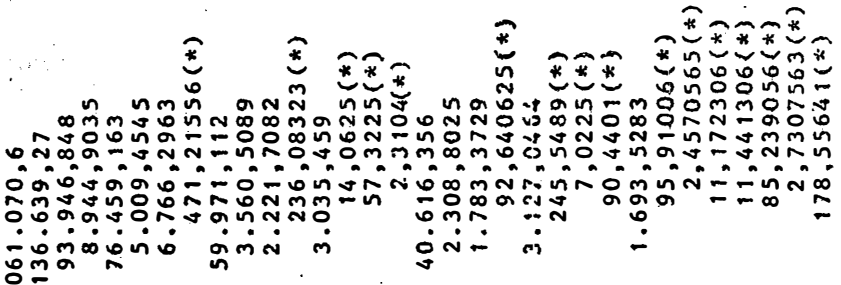

í

అ

ป

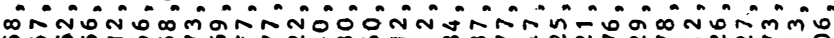

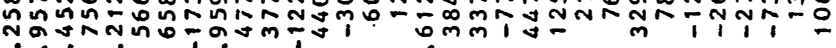
$\ddot{\sim} \dot{\sim} \dot{\sim}$

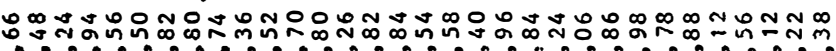

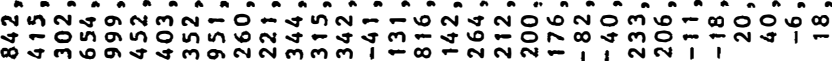
$\dot{a} \div \dot{0}$

ஸ⿻

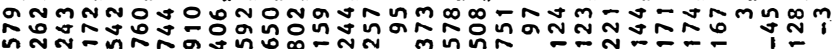
$\dot{\Delta} \dot{0} \dot{0}$

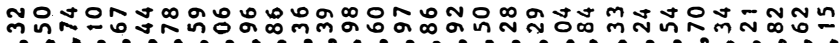
일

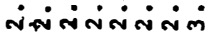

চ

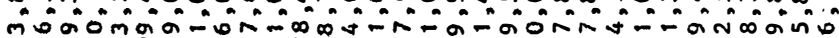

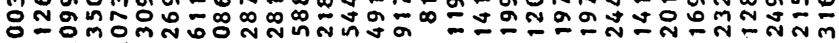

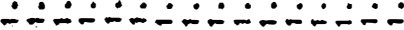

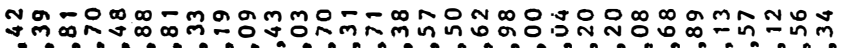

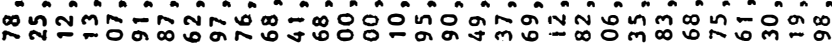

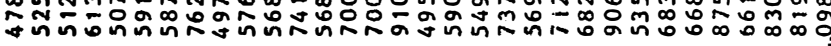

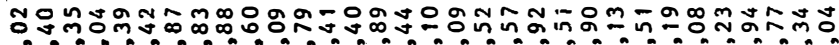
స N

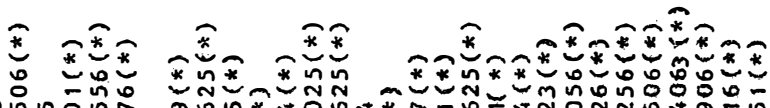
กำกำ

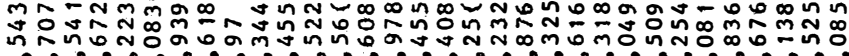

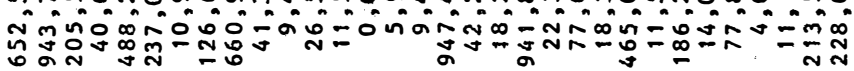
$\dot{m}-\dot{m}-\dot{-}$

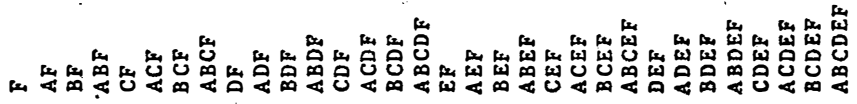

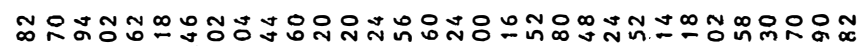

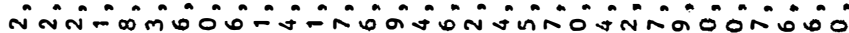

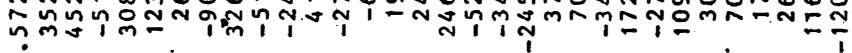
- .

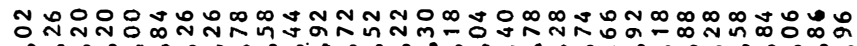
mon

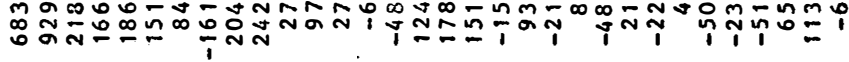
운

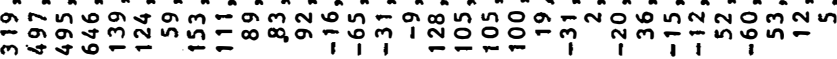

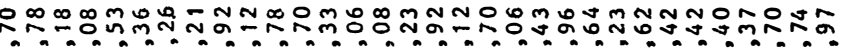

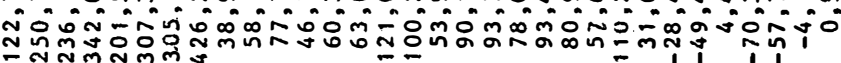

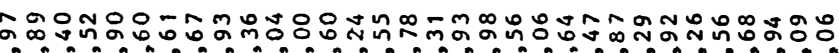
○ัด

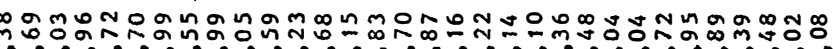

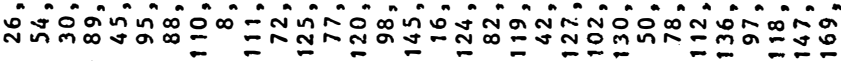

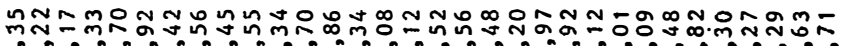
กิن

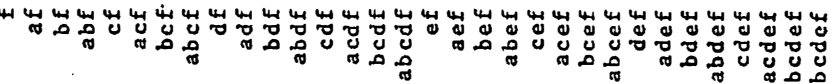


.187.

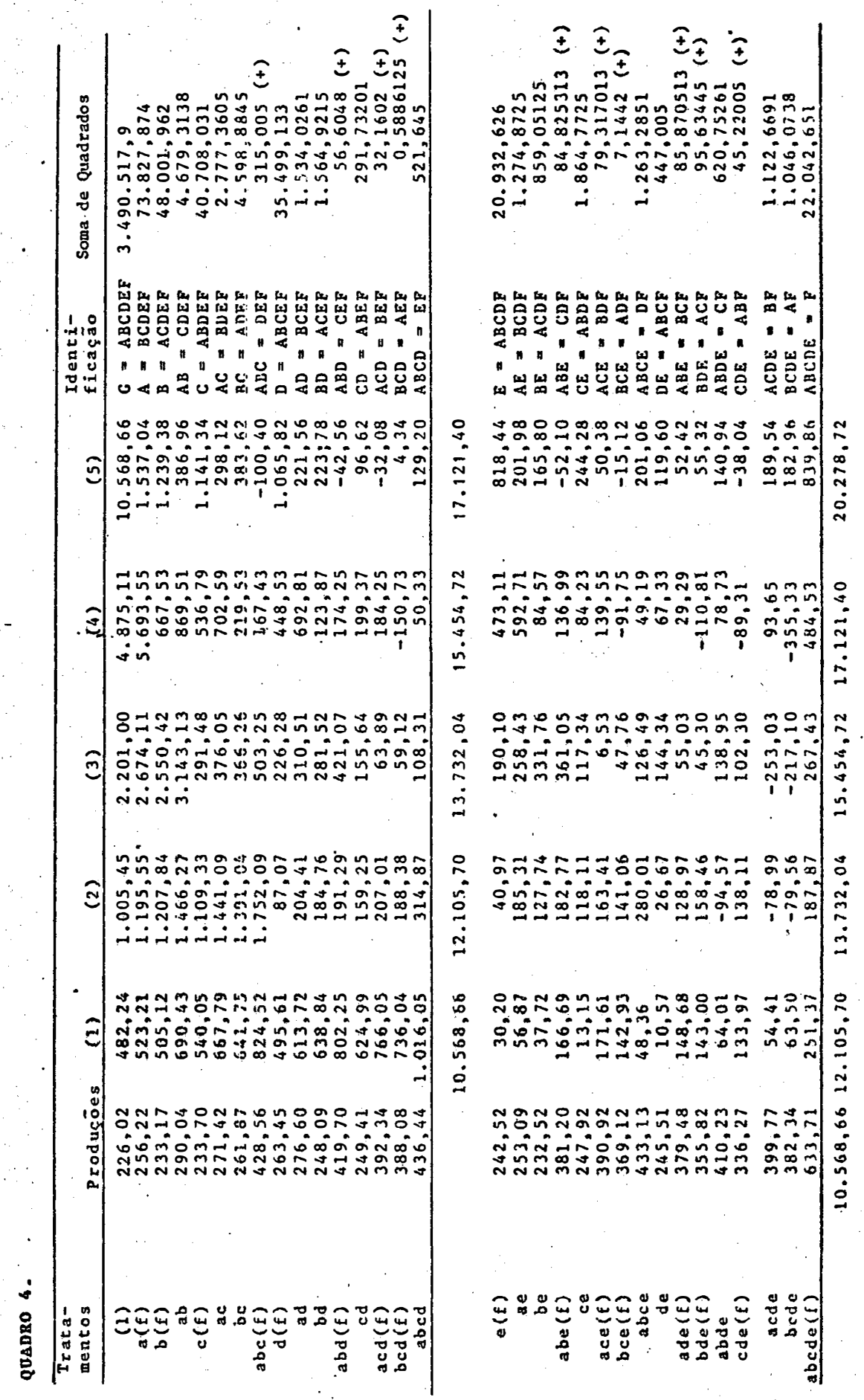




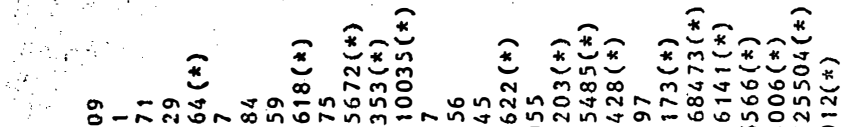

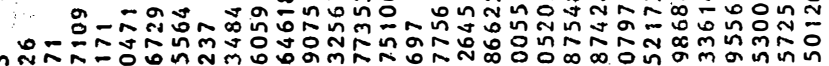

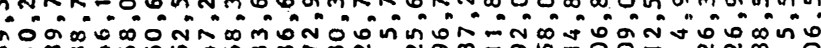

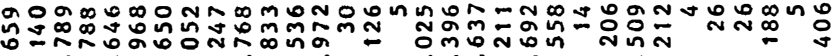

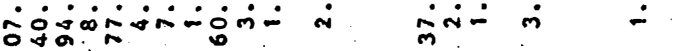
$\div$ r

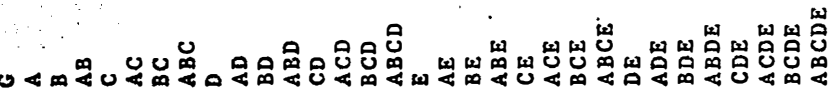

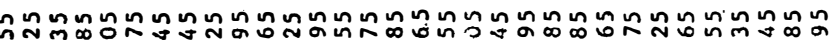

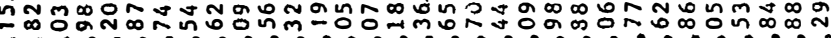

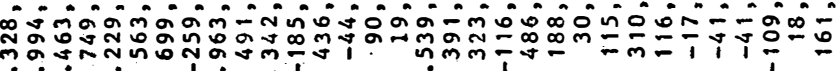

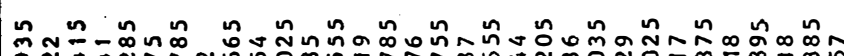
a.ง

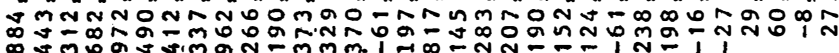

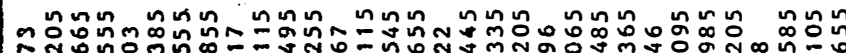
an

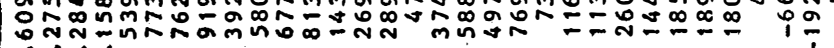
inisio

œ ษ तें लंखिंलि

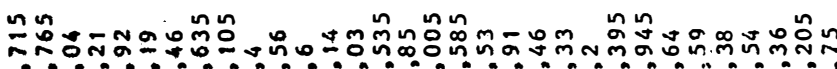

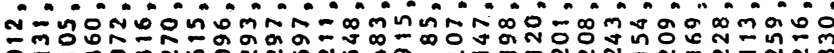

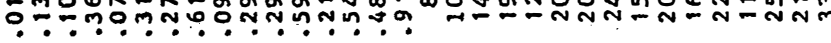

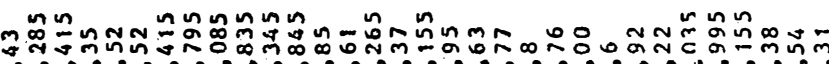

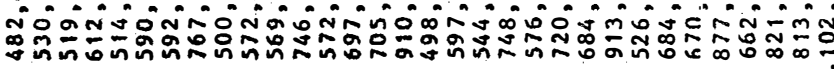

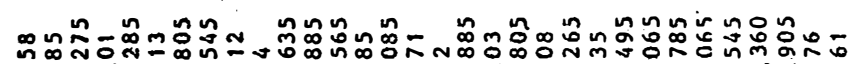

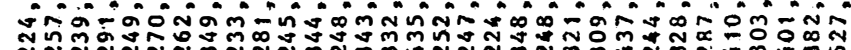

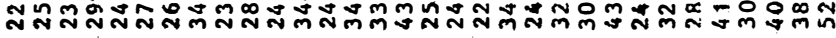

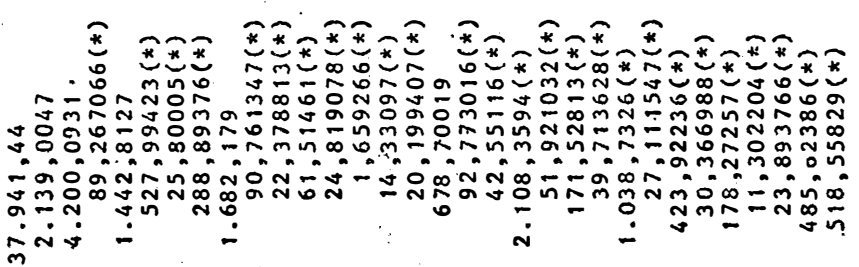

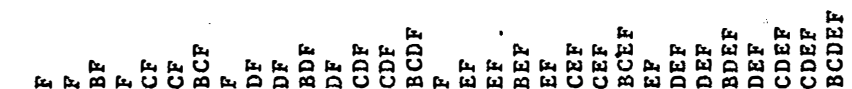

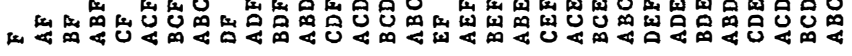

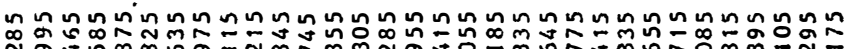

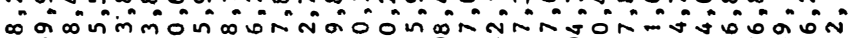
nิ

กัฒ

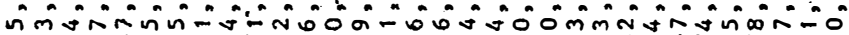
Oำ

ผ

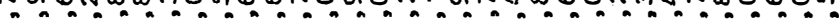

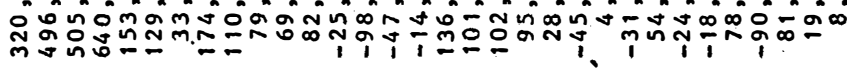

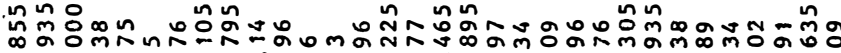

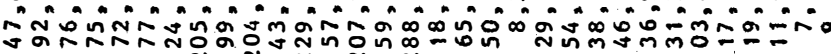

ำน mं -

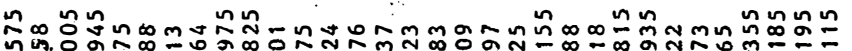

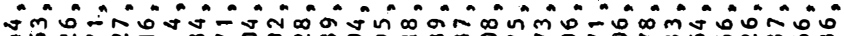

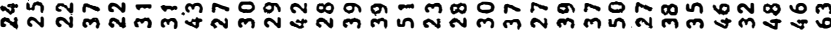

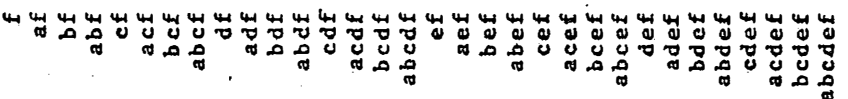




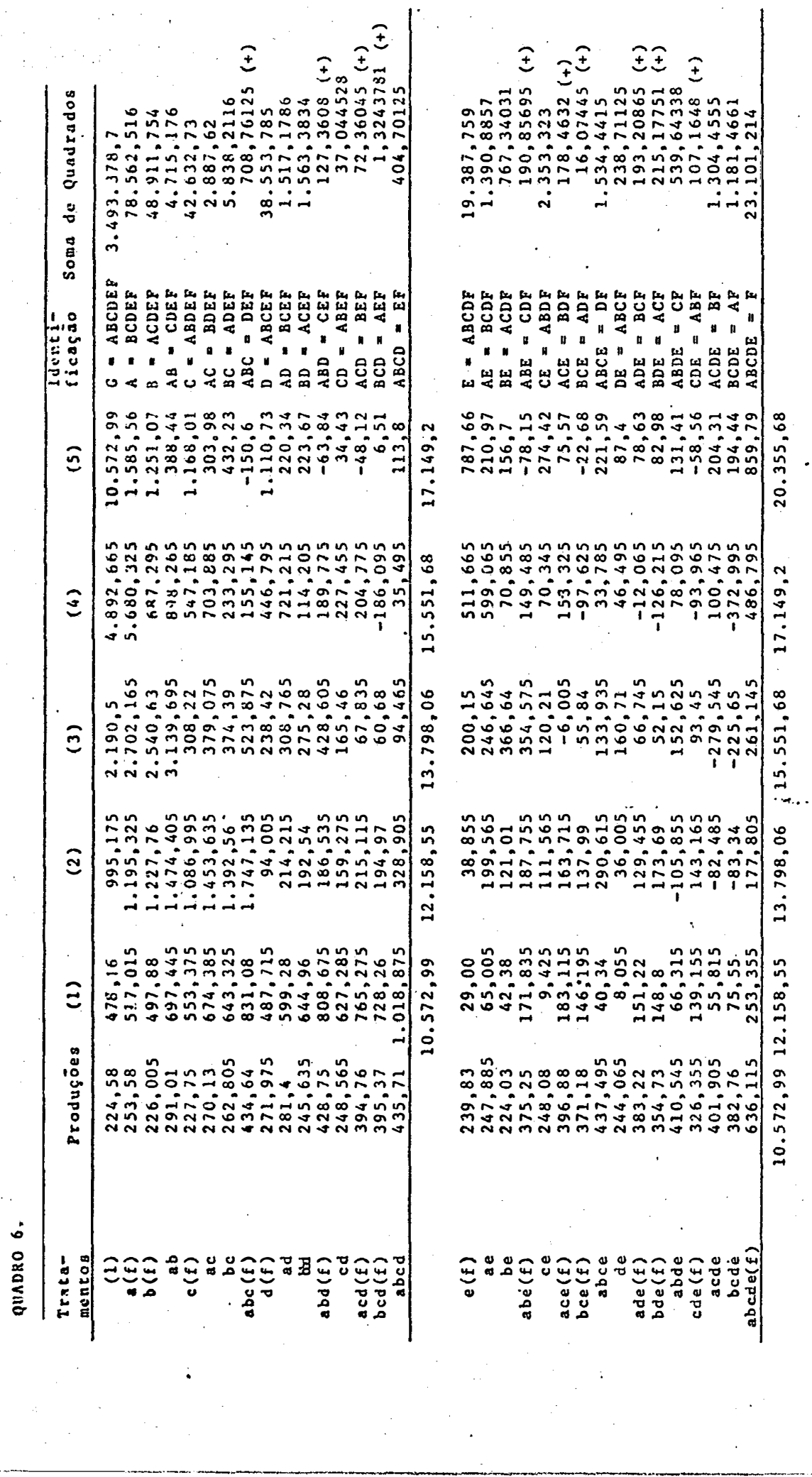




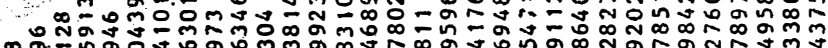
कon

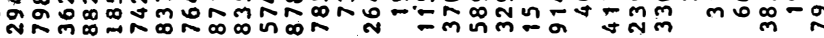

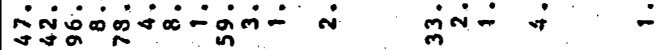
i

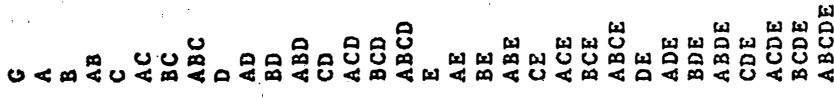

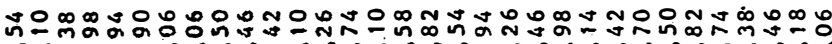

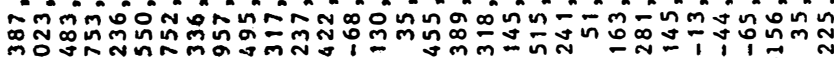
$\therefore \dot{i} \boldsymbol{i} \sim$

คํำ

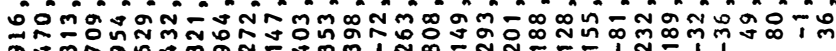

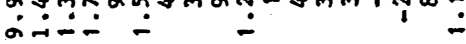

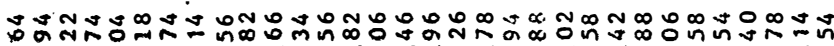
คิำง ำ

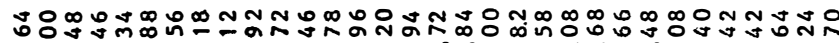

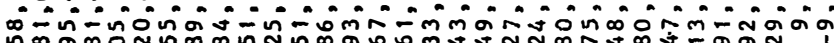

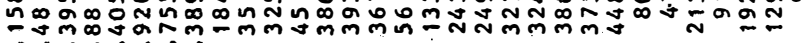

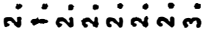

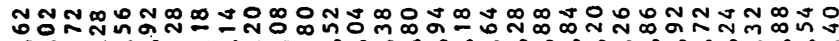

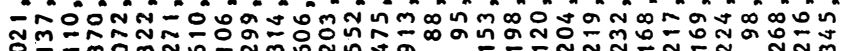

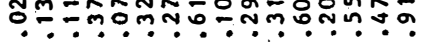

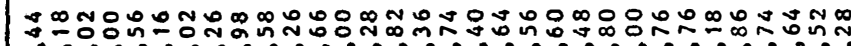

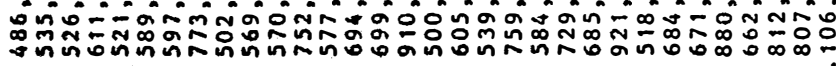

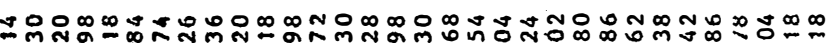

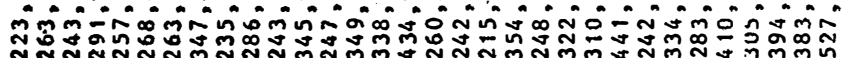

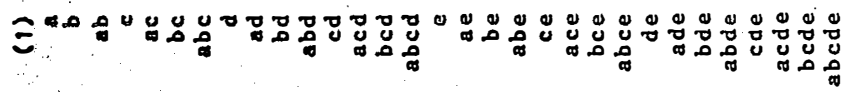

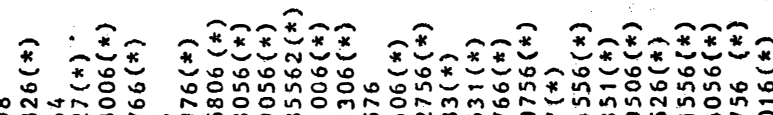

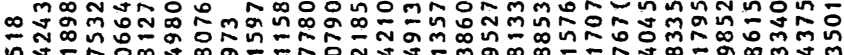
- 0 -

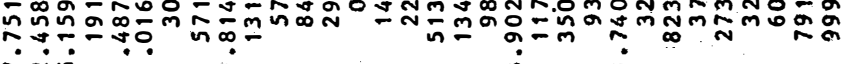
inin - :-

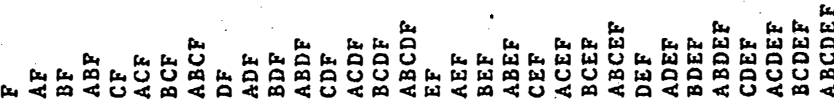

మొ

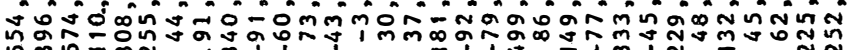

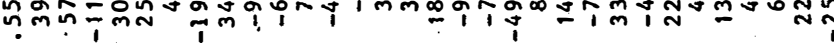

유ำ유

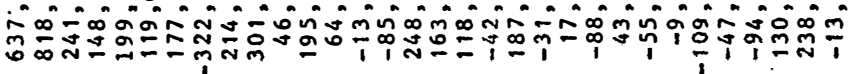

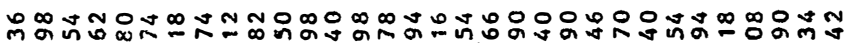

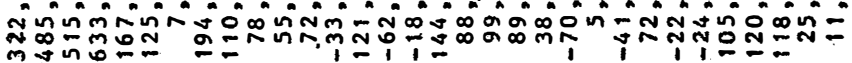

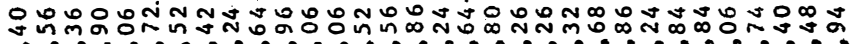

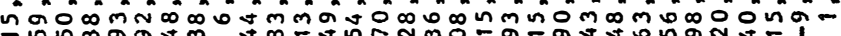

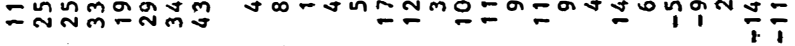

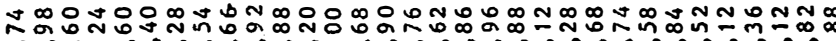

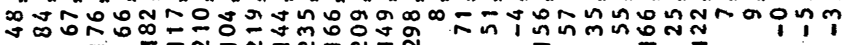

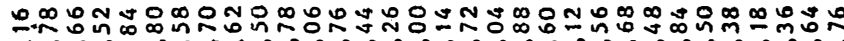

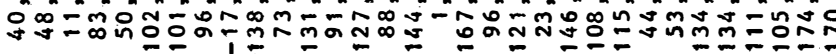

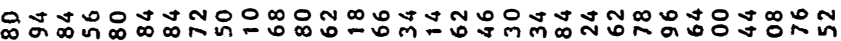

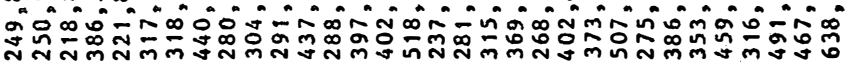

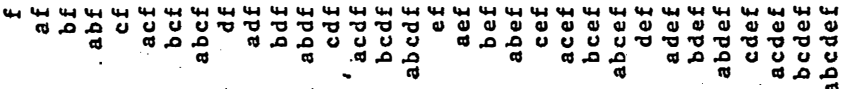



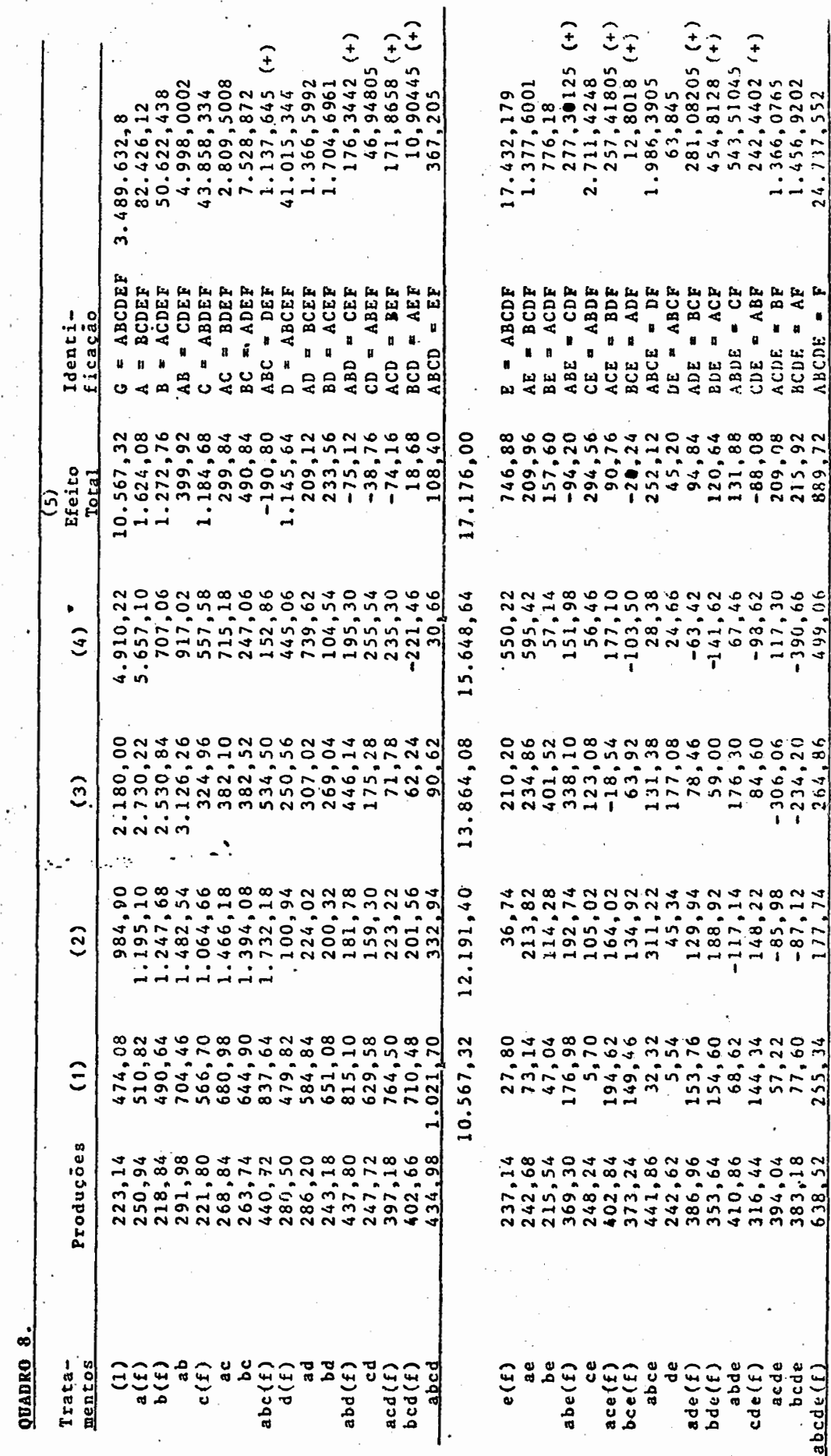

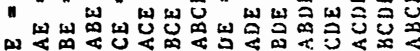

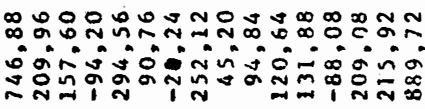

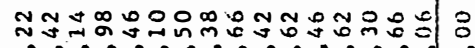

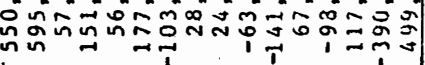

ㅇํํํㅇㅇำ

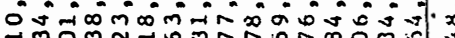

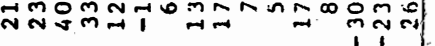

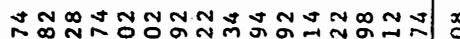

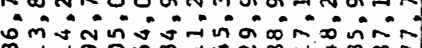
N

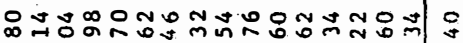

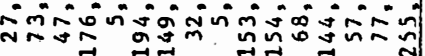

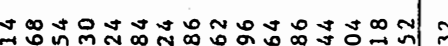

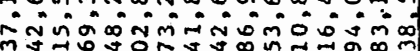
Nָสี 
.192 .

A N EXO 2 


\section{$3 \mathrm{CLS}$}

$5 \quad \mathrm{PA}=44539$

8 POKE 65495,0

$10 \mathrm{~N}=2$

$20 \mathrm{DIM} A(\mathrm{~N}), \mathrm{B}(\mathrm{N}), \mathrm{C}(\mathrm{N}), \mathrm{D}(\mathrm{N}), \mathrm{E}(\mathrm{N}), \mathrm{F}(\mathrm{N}), \mathrm{AB}(\mathrm{N}, \mathrm{N}), \mathrm{AC}(\mathrm{N}, \mathrm{N}), \mathrm{BC}(\mathrm{N}, \mathrm{N})$, $\operatorname{AD}(\mathrm{N}, \mathrm{N}), \mathrm{BD}(\mathrm{N}, \mathrm{N}), \mathrm{CD}(\mathrm{N}, \mathrm{N}), \operatorname{AE}(\mathrm{N}, \mathrm{N}), \mathrm{BE}(\mathrm{N}, \mathrm{N}), \mathrm{CE}(\mathrm{N}, \mathrm{N}), \mathrm{DE}(\mathrm{N}, \mathrm{N})$, $\operatorname{AF}(\mathrm{N}, \mathrm{N}), \mathrm{BF}(\mathrm{N}, \mathrm{N}), \mathrm{CF}(\mathrm{N}, \mathrm{N}), \mathrm{DF}(\mathrm{N}, \mathrm{N}), \mathrm{EF}(\mathrm{N}, \mathrm{N})$,

$30 \operatorname{DIM} A 1(N, N, N), A 2(N, N, N), A 3(N, N, N) B 1(N, N, N), A 4(N, N, N)$, $A 5(N, N, N), B 2(N, N, N), A 6(N, N, N), B 3(N, N, N), C 1(N, N, N)$, $A 7(N, N, N), A 8(N, N, N), B 4(N, N, N), A 9(N, N, N), B 5(N, N, N)$, $C 2(N, N, N), C 3(N, N, N), B 6(N, N, N), C 4(N, N, N), D 1(N, N, N)$,

$40 \operatorname{DIM} B 7(\mathrm{~N}, \mathrm{~N}, \mathrm{~N}, \mathrm{~N}), \mathrm{B} 8(\mathrm{~N}, \mathrm{~N}, \mathrm{~N}, \mathrm{~N}), \mathrm{B} 9(\mathrm{~N}, \mathrm{~N}, \mathrm{~N}, \mathrm{~N}), \mathrm{C} 5(\mathrm{~N}, \mathrm{~N}, \mathrm{~N}, \mathrm{~N})$ $\mathrm{C} 6(\mathrm{~N}, \mathrm{~N}, \mathrm{~N}, \mathrm{~N}), \mathrm{C} 7(\mathrm{~N}, \mathrm{~N}, \mathrm{~N}, \mathrm{~N}), \mathrm{C} 8(\mathrm{~N}, \mathrm{~N}, \mathrm{~N}, \mathrm{~N}), \mathrm{C} 9(\mathrm{~N}, \mathrm{~N}, \mathrm{~N}, \mathrm{~N})$, $\mathrm{D} 2(\mathrm{~N}, \mathrm{~N}, \mathrm{~N}, \mathrm{~N}), \mathrm{D} 3(\mathrm{~N}, \mathrm{~N}, \mathrm{~N}, \mathrm{~N}), \mathrm{D} 4(\mathrm{~N}, \mathrm{~N}, \mathrm{~N}, \mathrm{~N}), \mathrm{D} 5(\mathrm{~N}, \mathrm{~N}, \mathrm{~N}, \mathrm{~N})$, D $6(N, N, N, N), D 7(N, N, N, N), D 8(N, N, N, N)$

$50 \operatorname{DIM} D 9(\mathrm{~N}, \mathrm{~N}, \mathrm{~N}, \mathrm{~N}, \mathrm{~N}), \mathrm{E} 1(\mathrm{~N}, \mathrm{~N}, \mathrm{~N}, \mathrm{~N}, \mathrm{~N}), \mathrm{E} 2(\mathrm{~N}, \mathrm{~N}, \mathrm{~N}, \mathrm{~N}, \mathrm{~N})$, $\mathrm{E} 3(\mathrm{~N}, \mathrm{~N}, \mathrm{~N}, \mathrm{~N}, \mathrm{~N}), \mathrm{E} 4(\mathrm{~N}, \mathrm{~N}, \mathrm{~N}, \mathrm{~N}, \mathrm{~N}), \mathrm{E} 5(\mathrm{~N}, \mathrm{~N}, \mathrm{~N}, \mathrm{~N}, \mathrm{~N})$,

$60 \operatorname{DIM} E 6(\mathrm{~N}, \mathrm{~N}, \mathrm{~N}, \mathrm{~N}, \mathrm{~N}, \mathrm{~N}), \mathrm{E} 7(\mathrm{~N}, \mathrm{~N}, \mathrm{~N}, \mathrm{~N}, \mathrm{~N}, \mathrm{~N}), Y(\mathrm{~N}, \mathrm{~N}, \mathrm{~N}, \mathrm{~N}, \mathrm{~N}, \mathrm{~N})$

70 INPUT "MEDIA"="; $M$

80 FOR $\mathrm{I}=1$ TO $\mathrm{N}$

90 PRINT "A" I"=";: INPUT A (I)

100 PRINT "B"I"=";: INPUT B(I)

110 PRINT "C"I"=; : INPUT C (I)

120 PRINT "D"I"=";: INPUT D(I)

130 PRINT "E"I"=";: INPUT E(I)

150 PRINT "F"I"="; : INPUT F(I)

$160 \mathrm{NEXT} \mathrm{I}$

170 FOR I $=1$ TO N

180 FOR J=1 TO N

190 PRINT "AB"I"J"="; : $\operatorname{INPUT} A B(I, J)$

200 PRINT "AC"I"J"="; : INPUT AC $(I, J)$

210 PRINT "AD"I"J"=";: INAUT $\operatorname{AD}(I, J)$

220 PRINT "AE"I"J"="; : INPUT AE $(I, J)$ 230 PRINT "AF"I"J"="; : INPUT $\operatorname{AF}(I, J)$ 
240 PRINT "BCC"I"J":=";: INPUT BC(I, J )

250 PRINT. "BD" I"J"=";: INPUT BD(I,J)

260 PRINT "BE".I""J""=";: INPUT BE $(I, J)$

270 PRINT "BF"I""J"=";: $\operatorname{INPUT} \dot{B} F(I, J)$

280 PRINT "CD"I""J"=!'; : ' INPUT CD $(\mathrm{I}, \mathrm{J})$

290 PRINT. "CE"I"!"J"=" ; : INPUT' CE $(\mathrm{I} ; \mathrm{J})$

300 PRINT "CF"I"!"J"=";: INPUT CF $(\mathrm{I}, \mathrm{J})$

310 PRINT "DE"I":J"=";: INPUT DE(I, J )

320 PRINT "DF"I"!J"!=":;: INPUT: DF (I, J )

330 PRINT "EF"I"!"y" =", ; : INPUT EF (I,J)

$340 \mathrm{NEXT} \mathrm{J}, \mathrm{I}$

350 FOR $I=1$ TO N

360 FOR $\mathrm{J}=1$ TO $\mathrm{N}$

370 FOR $\mathrm{K}=1$ TO N

380 PRINT. "ABC" I"" J"" K" =" ; : INPUT A $1(\mathrm{I}, \mathrm{J}, \mathrm{K})$

390 PRINT "ABD"I" "J"."K"="; : INPUT A2 ( I, J,K)

400 PRINT "ABE"I" "J" "K"="; : INPUT A.4 ( I, J,K)

410 PRINT "ABF"I" "J" "K"=";: INPUT A $7(I, J, K)$

420 PRINT "ACD"I" "J""K"=";: INPUT A.3 ( I, J,K)

430 PRINT "ACE"I" "J" $K "="$; : INPUT A $5(I, J, K)$

440 PRINT "ACF"I" "J" "K"=";: INPUT A8 ( I, J,K)

450 PRINT "ADE"I" "J" $K "="$; : INPUT A6 (I,J,K)

460 PRINT "ADF"I" "J""K" $=$; : INPUT A.9 (I,J.K)

470 PRINT "AEF"I" "J" $K "="$; : INPUT C $3(\mathrm{I}, \mathrm{J}, \mathrm{K})$

480 PRINT "BCD"I" "J" "K"=";: INPUT $B I(I, J, K)$

490 PRINT. "BCE"I" "J" "K"=", ; : INPUT B $2(\mathrm{I}, \mathrm{J}, \mathrm{K})$

500 PRINT "BCF"I" "J" "K"=";: INPUT B4 ( I, J,K)

510 PRINT. "BDE"I" "J" "K"=" ; : INPUT. B $3(\mathrm{I}, \mathrm{J}, \mathrm{K})$

520 PRINT "BDF"I" "J""K"=";: INPUT B $5(\mathrm{I}, \mathrm{J}, \mathrm{K})$

530 PRINT "BEF"I" "J""K"=";: INPUT B6 ( I, J,K)

560 PRINT. "CDE"I" "J" "K"=";: INPUT CI ( I, J,K)

570 PRINT. "CDF"I" "J" "K"=";: INPUT C $2(\mathrm{I}, \mathrm{J}, \mathrm{K})$

580 PRINT "CEF"I" "J" $K "="$; : INPUT C4 ( I, J,K)

590 PRINT "DEF"I" "J""K"=";: INPUT DI ( I, J,K)

$600 \mathrm{NEXT} \mathrm{K}, \mathrm{J}, \mathrm{I}$ 
610 FOR I= 1 TO N

620 FOR J $=1$ TO N

630 FOR $\mathrm{K}=1$ TO $\mathrm{N}$

640 FOR L= 1 TO N

650 PRINT "ABCD!" "I" "J." "K" "L" = ";: INPUT B $7(\mathrm{I}, \mathrm{J}, \mathrm{K}, \mathrm{L})$

660 PRINT "ABCE" I" "J""K""L"="; : INPUT B8 ( I, J,K,L)

670 PRINT "ABCF""I" "J""K""L"=";: INPUT C7 ( I, J,K, L)

680 PRINT "ABDE""I" "J""K""L"="; : INPUT B9( I, J, K, L)

690 PRINT "ABDF" "I" "J" "K" "L"=";: INPUT C $8(\mathrm{I}, \mathrm{J}, \mathrm{K}, \mathrm{L})$

700 PRINT "ABEF" "I" "J"K"K"L"=";: INPUT D3 ( I, J, K, L )

710 PRINT "ACDE" I"I"J""K" "L"="; : INPUT C $5(\mathrm{I}, \mathrm{J}, \mathrm{K}, \mathrm{L})$

720 PRINT "ACDF" "I" "J""K" "L"="; : INPUT C $9(\mathrm{I}, \mathrm{J}, \mathrm{K}, \mathrm{L})$

730 PRINT "ACEF" "I" "J""K" "L"=";: INPUT D4 ( I, J, K, L )

740 PRINT "ADEF""I""J" "K" "L"=";: INPUT D6(I,J,K, L)

750 PRINT "BCDE"!! I" "J""K" "L"=";: INPUT C6( I, J, K, L)

760 PRINT. "BCDF""I" "J""K" "L"="; : INPUT D2( I, J,K, L)

770 PRINT "BCEF""I" "J" "K" "L"=";: INPUT D5( I, J, K, L)

780 PRINT "BDEF""I" "J" "K""L"=";: INPUT D I ( I, J, K, L )

790 PRINT "CDEF""I""J""K" "L"="; : INPUT D8( I, J,K, L)

$800: \mathrm{NEXT} L, \mathrm{~K}, \mathrm{~J}, \mathrm{I}$

810 FOR $\mathrm{I}=1$ TO N

820 FOR J $=1$ TO N

830 FOR $\mathrm{K}=1$ TO $\mathrm{N}$

840 FOR $\mathrm{L}=1$. TO N

850 FOR $D=1$ TO N

860 PRINT "ABCDE." "I" "J" "K" "L." "O"="; : INPUT D $9(\mathrm{I}, \mathrm{J}, \mathrm{K}, \mathrm{L}, \mathrm{O})$

870 PRINT "ABCDF" "I""J" "K" "L" "O"=";: INPUT EI ( I, J, K, L, O)

880 PRINT "ABCEF" "I" "J" "K" "L""O"="; : INPUT E2 ( I, J , K, L , O )

890 PRINT "ABDEF" I" "J" "K" "L" "O"=";: INPUT E3 ( I, J, K, L, O)

900 PRINT "ACDEF" "I" "J" "K" "L""O"

910 PRINT "BCDEF" I" "J" "K" "L" "O" $=$ "; : INPUT E $5(\mathrm{I}, \mathrm{J}, \mathrm{K}, \mathrm{L}, \mathrm{O})$

$920 \mathrm{NEXT} 0, \mathrm{~L}, \mathrm{~K}, \mathrm{~J}, \mathrm{I}$

930 FOR $\mathrm{I}=1$ TO N

940 FOR J=1 TO N 
950 FOR K=1 TQ N

960 FOR J $=1$ TO N

970 FOR $O=1$ TO N

980 FOR $P=1$ TO N

990 PRINT "ABCDEF"I""J""K" "L""O" "P"="; : INPUT E $6(\mathrm{I}, \mathrm{J}, \mathrm{K}, \mathrm{L}, \mathrm{O}, \mathrm{P})$

1000 PRINT "ERRO"I"J"K"L"O"P"=";: INPUT E 7 ( I, J, K, L, O,P)

$1010 \mathrm{NEXT}$ P, O,L,K,J, I

1012 CLS: INPUT" DESEJA MULTIPLICAR O ERRO POR UMA CONSTANTE $<\mathrm{S} / \mathrm{N}>$; $\mathrm{S} \$$

1014 IF $\mathrm{S} \$=$ "S" THEN PRINT "CONSTANTE=";: INPUT KK

1016 IF $S \$=" N "$ THEN $K K=1$

1018 CLS: $Z=0$

1030 FOR $P=1$ TO N

1040 FOR $0=1$ TO $N$

1050 FOR L $=1$ TO $\mathrm{N}$

1060 FOR $\mathrm{K}=1$ TO N

1070 FOR J=1 TO N

1080 FOR $\mathrm{I}=1$ TO $\mathrm{N}$

$1090 \mathrm{Y}(\mathrm{I}, \mathrm{J}, \mathrm{K}, \mathrm{L}, \mathrm{O}, \mathrm{P})=\mathrm{M}+\mathrm{A}(\mathrm{I})+\mathrm{B}(\mathrm{J})+\mathrm{C}(\mathrm{K})+\mathrm{D}(\mathrm{L})+\mathrm{E}(\mathrm{O})+\mathrm{F}(\mathrm{P})+\mathrm{AB}(\mathrm{I}, \mathrm{J})+$ $A C(I, J)+B C(I, J)+A D(I, L)+B D(J, L)+C D(K, L)+A E(I, 0)+B E(J, 0)+$ $C E(K, 0)+D E(L, 0)+A F(I, P)+B F(J, P)+C F(K, P)+D F(L, P)+E F(0, P)+$ $A 1(I, J, K)+A 2(I, J, L)+A 3(I, K, L)+B 1(J, K, L)+A 4(I, J, O)+$ $A 5(I, K, O)+B 2(J ; K, O)$

$1100 Y(I, J, K, L, O, P)=Y(I, J, K, L, O, P)+A 6(I, L, O)+B 3(J, L, O)+$ $\mathrm{C} 1(\mathrm{~K}, \mathrm{~L}, \mathrm{O})+\mathrm{A} 7(\mathrm{I}, \mathrm{J}, \mathrm{P})+\mathrm{A} 8(\mathrm{I}, \mathrm{K}, \mathrm{P})+\mathrm{B} 4(\mathrm{~J}, \mathrm{~K}, \mathrm{P})+\mathrm{A} 9(\mathrm{I}, \mathrm{L}, \mathrm{P})+$ $\mathrm{B} 5(\mathrm{~J}, \mathrm{~L}, \mathrm{P})+\mathrm{C} 2(\mathrm{~K}, \mathrm{~L}, \mathrm{P})+\mathrm{C} 3(\mathrm{I}, \mathrm{O}, \mathrm{P})+\mathrm{B} 6(\mathrm{~J}, \mathrm{O}, \mathrm{P})+\mathrm{C} 4(\mathrm{~K}, \mathrm{O}, \mathrm{P})+$ $\mathrm{D} 1(\mathrm{~L}, \mathrm{O}, \mathrm{P})+\mathrm{B} 7(\mathrm{I}, \mathrm{J}, \mathrm{K}, \mathrm{L})+\mathrm{B} 8(\mathrm{I}, \mathrm{J}, \mathrm{K}, \mathrm{O})+\mathrm{B} 9(\mathrm{I}, \mathrm{J}, \mathrm{L}, \mathrm{O})+\mathrm{C} 5(\mathrm{I}, \mathrm{K}, \mathrm{L}, \mathrm{O})+$ $\mathrm{C} 6(\mathrm{~J}, \mathrm{~K}, \mathrm{~L}, \mathrm{O})+\mathrm{C} 7(\mathrm{I}, \mathrm{J}, \mathrm{K}, \mathrm{P})+\mathrm{C} 8(\mathrm{I}, \mathrm{J}, \mathrm{L}, \mathrm{P})$

$1110 \mathrm{Y}(\mathrm{I}, \mathrm{J}, \mathrm{K}, \mathrm{L}, \mathrm{O}, \mathrm{P})=\mathrm{Y}(\mathrm{I}, \mathrm{J}, \mathrm{K}, \mathrm{L}, \mathrm{O}, \mathrm{P})+\mathrm{C} 9(\mathrm{I}, \mathrm{K}, \mathrm{L}, \mathrm{P})+\mathrm{D} 2(\mathrm{~J}, \mathrm{~K}, \mathrm{~L}, \mathrm{P})+$ D3 $(I, J, 0, P)+D 4(I, K, 0, P)+D 5(J, K, O, P)+D 6(I, L, O, P)+$ $D 7(J, L, O, P)+D 8(K, L, O, P)+D 9(I, J, K, L, O)+E 1(I, J, K, L, P)+$ $\mathrm{E} 2(\mathrm{I}, \mathrm{J}, \mathrm{L}, \mathrm{O}, \mathrm{P})+\mathrm{E} 3(\mathrm{I}, \mathrm{J}, \mathrm{L}, \mathrm{O}, \mathrm{P})+\mathrm{E} 4(\mathrm{I}, \mathrm{K}, \mathrm{L}, \mathrm{O}, \mathrm{P})+\mathrm{ES}(\mathrm{J}, \mathrm{K}, \mathrm{L}, \mathrm{O}, \mathrm{P})+$ $E 6(I, J, K, L, O, P)+E 7(I, J, K, L, O, P)$ 


\section{$1120 \mathrm{Z}=\mathrm{Z}+1$}

1130 IF $Z=15$ THEN EXEL PA

1140 IF $Z=15$ THEN $Z=0$

1150 PRINT "Y"'"I""J""K""L!" "O""P") =" ; Y ( I , J , K, , L , O, P )

1160 NEXT I, J, K, L, O,P

1170 INPUT DESEJA GRAVAR OS DADOS EM FITA <S/N>";:S\$

1180 IF $S \$=$ "N" THEN 1330

\section{CLS}

1200 INPUT" COLOQUE A FITA, PRESSIONE PLAY/RECORD E <ENTER>"; $\$$

1210 OPEN "O",\#-1, " "

1220 FOR $P=1$ TO $N$

1230 FOR $O=1$ TO $N$

1240 FOR L=1 TO N

1250 FOR $K=1$ TO N

1260 FOR J=1 TO $\mathrm{N}$

1270 FOR I $=1$ TO N

1280 PRINT $\#-1, Y(I, J, K, L, 0, P)$

1290 NEXT I, J, K, L, O,P

1300 CLOSE \#-1

$1310 \mathrm{CLS}$

1320 PRINT"DADOS GRAVADOS" : EXEC PA

1330 POKE 65494,0

1340 CLS : PRINT a 237,"FIM"

1350 END 